\title{
Robust Control of Rotordynamic Instability in Rotating Machinery Supported by Active Magnetic Bearings
}

\author{
A Dissertation \\ Presented to \\ the faculty of the School of Engineering and Applied Science \\ University of Virginia
}

In Partial Fulfillment

of the requirements for the degree

Doctor of Philosophy

Electrical Engineering

by

Simon Estomih Mushi

May 2012 
(C) Copyright May 2012

Simon Estomih Mushi

All Rights Reserved 


\section{APPROVAL SHEET}

The dissertation

is submitted in partial fulfillment of the requirements

for the degree of

Doctor of Philosophy

(Electrical Engineering)

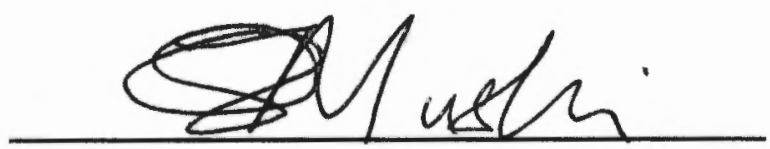

Simon E. Mushi

The dissertation has been read and approved by the examining committee
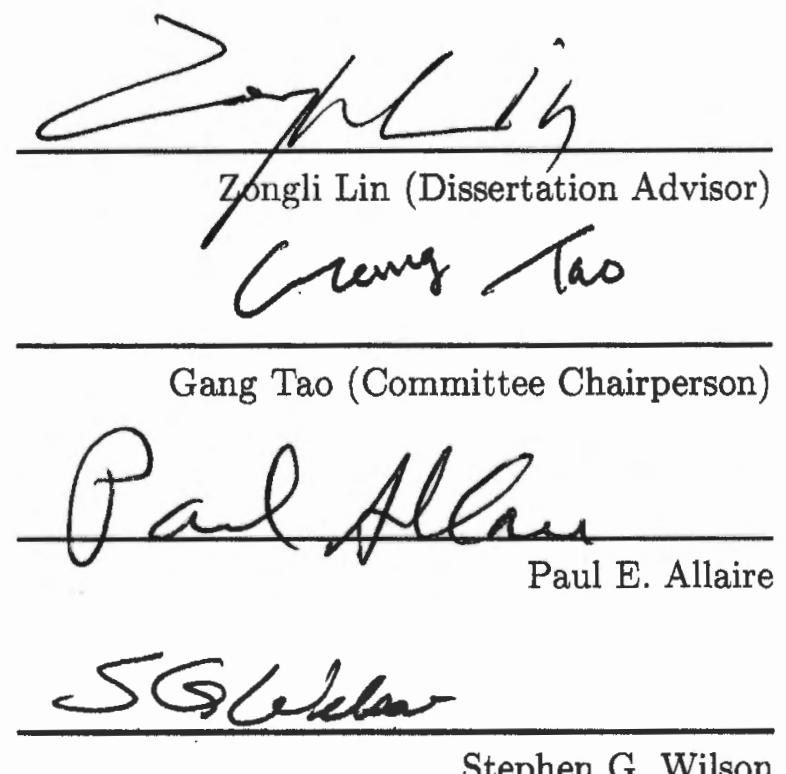

Stephen G. Wilson

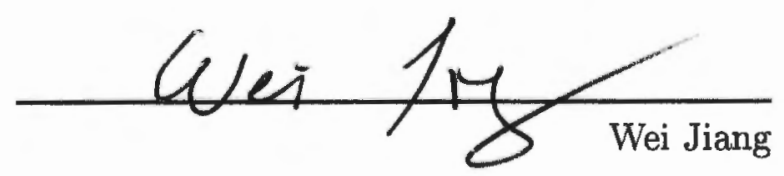

Accepted for the School of Engineering and Applied Science:
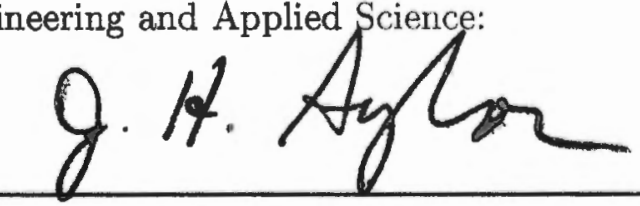

Dean, School of Engineering and Applied Science 


\begin{abstract}
This dissertation addresses the control challenges for a practical mechatronic system subject to self-excited instability modeled as a parametric uncertainty. Achieving a balance between the conflicting requirements of performance and robustness in the face of system uncertainty is the primary objective of feedback control. Practical issues such as unstable open-loop plant dynamics, finite actuator capacity, the presence structural flexibility and suboptimal sensor/actuator placement limit the achievable performance through the use of feedback.

The ROMAC Magnetic Bearing Test Rig for Rotordynamic Instability (MBTRI) is a state-of-the-art experiment designed to investigate algorithms that may affect the region of stability of a rotor-bearing system with respect to rotordynamic instability as a result of aerodynamic cross-coupled stiffness. The onset of rotordynamic instability is a significant challenge to successful design and operation of high speed rotating machinery particularly gas compressors. The unique design of the test rig includes several features of an industrial centrifugal gas compressor with a flexible rotor designed to operate above its first bending critical speed. The impellers and gas seals within compressors are the primary source of load-dependent aerodynamic cross-coupled stiffness forces which can lead to self-excited instability and serious machine damage in the absence of sufficient support damping. During the design phase of rotating machines the accurate prediction of the onset of instability is made difficult by reliance on semi-empirical dynamic models with significant uncertainty. Literature on the rotordynamic instability mechanism reveals that in the presence of optimum support damping, a maximum achievable stability threshold can be derived as a function of physical parameters of the rotor-bearing system. This presents an ideal opportunity for the exploration of optimal robust active vibration control algorithms using active
\end{abstract}


magnetic bearings (AMBs). A notable advantage of AMBs is their ability to generate optimal support stiffness and damping characteristics. Unlike passive mechanical bearings, the support characteristics of AMBs may be modified over the operating life of the system without any major hardware changes.

A general framework is presented whereby properties of the rotor-AMB system that impose fundamental limitations on achievable performance of the closed-loop system are evaluated as a nominal model of the MBTRI plant dynamic is constructed. This model was validated using system identification techniques, and augmented with uncertainty models representing the effects of variation in parameters such operating speed and the magnitude of the destabilizing stiffness. Using $\mu$-synthesis several robust controllers were designed and implemented on the MBTRI hardware to investigate their effect on the stability threshold. The best controller established a thirty-six percent increase in the stability threshold over an existing benchmark controller. This represented an increase from fifty-six percent of the maximum achievable stability threshold to seventy-six percent. Robust stability and performance analysis was performed to discern the extent that either the engineering specifications for the desired control performance or uncertainty model or a combination of the two may be altered to more closely approach the maximum stability threshold. 
To My Family 


\section{Acknowledgments}

The author would like to express his most sincere appreciation to Prof. Zongli Lin and Prof. Paul Allaire for their advice, insight, and encouragement during his graduate studies at the University of Virginia. The author has benefited significantly from their generosity and positive attitudes to research, engineering and life. Working, learning and growing under their guidance has been a superbly rewarding experience.

The author would like to also extend thanks to his dissertation committee members, Prof. Stephen G. Wilson, Prof. Gang Tao and Dr. Wei Jiang, for their enthusiasm and timely advice during the defense of his proposal and final dissertation.

Special thanks go to all the people in the Rotating Machinery and Controls (ROMAC) Laboratory which has become a second home to the author for the last few years. Rarely do experiments work the first time around, and without the countless helpful discussions and interventions with fellow lab members, this work could not have been completed. Thank you!

The author would like to thank his mother and father (the first two Dr. Mushi's he knew), his sister (the third) and his brothers (waiting in the wings) for their sacrifices, inspiration, and limitless support during his scholastic journey. This work is dedicated to them.

The author would like to reserve his final thanks and gratitude to his fiancée Olivia Mullins for her patience and encouragement and for adding a sparkle to his time in Charlottesville. 


\section{Contents}

1 Problem Statement 1

1.1 Introduction . . . . . . . . . . . . . . . . . . 1

1.2 Objectives ........................... 6

1.3 Contributions ...................... 7

1.4 Outline of the Dissertation . . . . . . . . . . . . 7

2 Literature Review 9

2.1 Rotordynamic Instability . . . . . . . . . . . . . . . . . . . 9

2.2 Rotor Vibration Control using AMBs . . . . . . . . . . . . . . . 12

2.2.1 General . . . . . . . . . . . . . . . . 12

2.2.2 Rotating Machinery Applications . . . . . . . . . . . . . 14

3 Rotordynamic Instability $\quad 18$

3.1 A Mechanical Systems Perspective . . . . . . . . . . . . . . . . . . 19

3.1 .1 Whirl Instability . . . . . . . . . . . . . . . . . . 19

3.1.2 Experimental Stability Measurement . . . . . . . . . . . . . . 22

3.1.3 Factors Affecting the Onset of Instability . . . . . . . . . . 26

3.2 A Control Systems Perspective . . . . . . . . . . . . . . . 28

3.2.1 Rotor-AMB System Description . . . . . . . . . . . . . . . . 28

3.2.2 Fundamental Control Limitations . . . . . . . . . . . . . . . 33

3.2.3 Unique Limitations of AMBs . . . . . . . . . . . . . . . 39

3.2.4 Stability Analysis . . . . . . . . . . . . . . . . 44

3.3 Summary ........................... 47

4 MBTRI Description and Modeling $\quad 50$

4.1 Test Rig Design . . . . . . . . . . . . . . . . . . . 50

4.1 .1 Overview ........................ 50

4.1.2 Rotor Construction ................. 52 
4.1.3 Electrical Components . . . . . . . . . . . . . . . 53

4.2 Component Models . . . . . . . . . . . . . . . . . 58

4.2 .1 Rotor Modeling . . . . . . . . . . . . . . . 58

4.2 .2 Actuator Modeling . . . . . . . . . . . . . . . . . 67

4.2.3 Modeling of Additional Components . . . . . . . . . 76

4.3 Reduced Order Model . . . . . . . . . . . . . . . . . . . . . . . . . . . . 79

4.4 Validation using System Identification $\ldots \ldots \ldots$

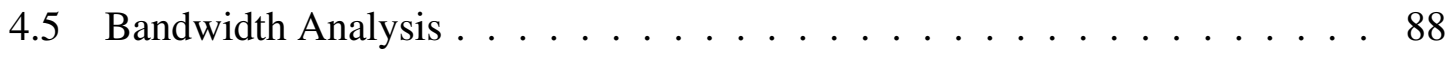

4.5.1 Available Bandwidth . . . . . . . . . . . . . . 88

4.5.2 Generating Cross-Coupled Stiffness _ . . . . . . . . . . . 89

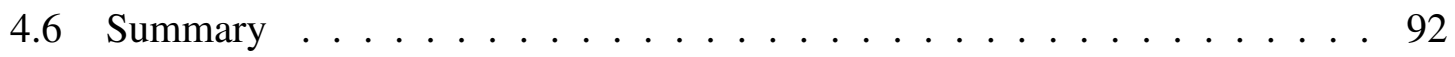

5 Robust Control Design and Analysis $\quad 94$

5.1 Objectives . . . . . . . . . . . . . . . . 95

5.2 Tools for Synthesis . . . . . . . . . . . . . . . . . . . 96

5.2.1 Structured Singular Value Framework _ . . . . . . . . . . 96

5.2.2 D-K iteration (Complex $\mu$-Synthesis) . . . . . . . . . . . 101

5.3 Uncertainty Models . . . . . . . . . . . . . . . . . . 103

5.3.1 Structured or Unstructured? . . . . . . . . . . . . . . . . 104

5.3.2 Uncertainty Model For Supercritical Operation . . . . . . . . . 105

5.3.3 Uncertainty Model For Enhancing Stability Threshold . . . . . . 109

5.4 Weighting Function Selection . . . . . . . . . . . . . . . 112

5.4.1 Weighting Function Template . . . . . . . . . . . . 118

5.4.2 Benchmark Controller . . . . . . . . . . . . . . . . 121

5.4 .3 Introducing Support Anisotropy . . . . . . . . . . . . 123

5.4.4 Introducing Nondiagonal Performance Weights . . . . . . . . 123

5.5 Synthesis Results . . . . . . . . . . . . . . . . . . 125

5.5.1 Evaluation of the Benchmark Controller . . . . . . . . . . 126

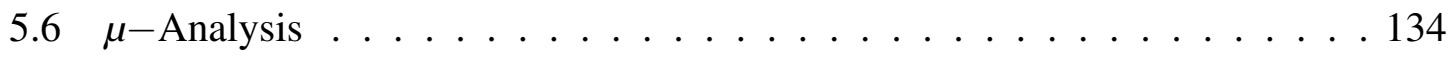

5.6.1 Controllers with Speed Uncertainty . . . . . . . . . . . . 135

5.6.2 Controllers with CCS Uncertainty . . . . . . . . . . . . . 137

5.7 Rotordynamic Analysis . . . . . . . . . . . . . . . . . . . . . 141

5.7.1 Forced Response to Unbalance . . . . . . . . . . . . . . . . 143

5.7.2 Stability (Damped Mode Shape) Analysis . . . . . . . . . . . 144

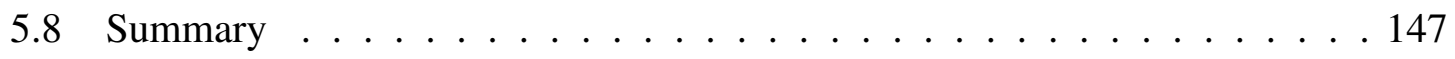


6 Experimental Results and Discussion $\quad 150$

6.1 Results . . . . . . . . . . . . . . . . . . 150

6.1.1 Supercritical Operation . . . . . . . . . . . . . . 150

6.1.2 System Transfer Functions . . . . . . . . . . . . . . . . 152

6.1.3 Stability Threshold ... . . . . . . . . . . . 158

6.2 Discussions . . . . . . . . . . . . . . . . . . . 168

6.2.1 Achievable versus Optimal Damping . . . . . . . . . . . . 168

6.2.2 Performance versus Uncertainty . . . . . . . . . . . . . . . . . . . 172

7 Conclusions and Recommendations $\quad 175$

7.1 Summary of Accomplishments . . . . . . . . . . . . . . 175

7.2 Recommendations for Further Work . . . . . . . . . . . . . . . . 177

$\begin{array}{lr}\text { Publications } & 180\end{array}$

$\begin{array}{ll}\text { References } & 180\end{array}$

A Test Rig Functional Overview 193

B v-Gap Metric for Robustness Analysis $\quad 195$

$\begin{array}{llr}\text { C } & \text { Experimental Stability Measurements } & 197\end{array}$

C.1 Hardware Setup . . . . . . . . . . . . . . . . . . . . . 197

C.2 Damping Ratio Estimation Algorithm . . . . . . . . . . . . . . . . . 198 


\section{List of Figures}

1.1 A multistage centrifugal compressor with upper casing removed showing the major components (Bidaut \& Baumann, 2010). . . . . . . . . . . 3

1.2 A waterfall plot of vibration spectra during startup of a high pressure centrifugal compressor. . . . . . . . . . . . . . . . . . 4

3.1 The force balance for a whirling shaft (Ehrich, 2004). . . . . . . . . 19

3.2 Predicted shaft motion at the onset of instability. . . . . . . . . . 22

3.3 Vibration amplitude spectrum of rotor demonstrating SSV as a result of CCS. 23

3.4 Waterfall plots of vibration spectra with and without CCS excitation. . . . . 24

3.5 The free decay of rotor vibrations following termination (indicated by the rising edge trigger signal) of forward circular blocking excitation while rotor spins at $7,000 \mathrm{rpm} . \ldots \ldots \ldots \ldots$

3.6 Pole-zero map of open-loop rotor-AMB system at $0 \mathrm{rpm}$ with and without

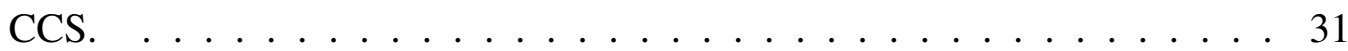

3.7 Pole-zero map of closed loop rotor-AMB system at $0 \mathrm{rpm}$ with and without CCS. . . . . . . . . . . . . . . . . . . . . . . 32

3.8 Magnitude plot of a typical sensitivity function. . . . . . . . . . . . . 34

3.9 A sensitivity function template for a plant with an unstable pole at $p_{1} \mathrm{rad} / \mathrm{s}$ and achievable bandwidth of $\Omega_{\mathrm{a}} \mathrm{rad} / \mathrm{s} . \ldots \ldots \ldots$

3.10 The loop gain of an arbitrary stable, minimum phase system showing the crossover frequency and the stability margins. . . . . . . . . . 38

3.11 A magnetization curve for silicon-iron showing the knee flux density of approximately $1.2 \mathrm{~T}$ (Meeker, 2009). . . . . . . . . . . . . . . . 41

3.12 Differential amplifier-AMB driving mode used for bias current linearization. 42

3.13 Magnitude plot illustrating the power bandwidth of an AMB-amplifier pair. 44

3.14 Mode shape plots generated during the rotor-AMB system stability analysis. 48 
4.1 A schematic showing the derivation of the test rig rotor design from a back-

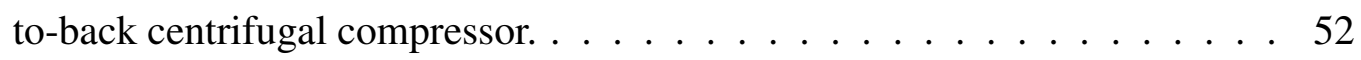

4.2 Machine drawings of the rotor (all units in inches). . . . . . . . . . 54

4.3 Drawing of the test rig assembly. . . . . . . . . . . . 57

4.4 Photograph of the completed test rig. . . . . . . . . . . . . . 57

4.5 A block diagram overview of rotor-AMB control system. . . . . . . . . 58

4.6 Hankel singular values of rotor-AMB model in modal space. . . . . . . . 62

4.7 Undamped critical speed map showing eigenvalues as a function of support stiffness. The closed-loop actuator stiffness for an exemplary controller is overlaid. . . . . . . . . . . . . . . . . . . 65

4.8 Free-free rotor bending mode shapes shown with respect to the sensor and actuator locations. . . . . . . . . . . . . . . . . . . 66

4.9 Campbell diagram with $5 \mathrm{MN} / \mathrm{m}$ support stiffness showing the variation of rotor eigenvalues with operating speed. . . . . . . . . . . 66

4.10 Plot of the difference between actuator force outputs determined using a linear magnetic circuit model and a two-dimensional finite element magnetostatic analysis for the NDE AMB . . . . . . . . . . . . 70

4.12 Equilibrium flux density in NDE AMB rotor, stator and air gap when only bias current $(4 \mathrm{~A})$ is flowing through all four quadrants. . . . . . . . . 71

4.11 Equilibrium field pattern developed in the NDE AMB stator and rotor when only the bias current $(4 \mathrm{~A})$ is flowing through all four quadrants. . . . . . 72

4.13 Field pattern developed in the NDE AMB stator and rotor with $I_{b}=4 \mathrm{~A}$ and $i_{p}=4$ A flowing in the top quadrants. . . . . . . . . . 73

4.14 Flux density in NDE AMB rotor, stator and air gap computed using 2-D finite elements with $I_{b}=4 \mathrm{~A}$ and $i_{p}=4 \mathrm{~A} . \ldots \ldots \ldots \ldots \ldots$

4.15 Dynamic force capacity as a function of frequency for the NDE and DE AMBs. . . . . . . . . . . . . . . . . . . . . 74

4.16 Front and side views of the radial AMBs showing materials and dimensions (air gap is not drawn to scale). Control quadrants are labeled 1-4 and shown in different colors. . . . . . . . . . . . . . . . . . . . 75

4.17 Normalized Bode plots of power amplifier response from command voltage input to AMB coil current output. The dash-dotted line is from the experiment and the solid line is from the model. . . . . . . . . . 78

4.18 Bode plots of the frequency response of the displacement sensor an eighth order inverse Chebychev anti-aliasing filter. . . . . . . . . . . . . 79 
4.19 Frequency response of sampling filters and computational delay approximation. . . . . . . . . . . . . . . . . 80

4.20 Bode plots of plant dynamics in the $x$-axis only. . . . . . . . . . . . . 83

4.21 Singular value plot of nominal plant model. . . . . . . . . . . . . . 84

4.22 Bode plot of phase lead filter used in PID controller. . . . . . . . . . . . . 86

4.23 Bode plot of PID controller for NDE-X and DE-X channels. . . . . . . . . 86

4.24 Bode plots comparing experimental frequency response to nominal plant model. . . . . . . . . . . . . . . . . . . . . . 87

4.25 Output sensitivity of NDEX and DEX channels of collocated PID controller. 89

4.26 Dynamic response of the feedback loop necessary to generate CCS. . . . . 92

5.1 Feedback connection illustrating the small gain theorem. . . . . . . . . . . 97

5.2 Standard plant-controller feedback connection for robust control. . . . . . . 98

5.3 Standard form for robust control. . . . . . . . . . . . . . . . . . . . . . 99

$5.4 \Delta-G-K$ feedback connection used during synthesis. . . . . . . . . . 101

5.5 Singular value plot of uncertain plant model illustrating variation in Nc3 and Nc4 natural frequencies. Compare with nominal plant model in Figure 4.21. . . . . . . . . . . . . . . . . . . 108

5.6 Bode plots of plant with uncertainty defined by CCS Model 1. . . . . . . 110

5.7 Singular value plot of plant with uncertainty defined by CCS Model 1. . . . 111

5.8 Bode plots of plant with uncertainty defined by CCS Model 3. . . . . . . 113

5.9 Singular value plot of plant with uncertainty defined by CCS Model 3, compare with Figure 5.7. . . . . . . . . . . . . . . . . . . . 114

5.10 Typical inverse performance weighting function, $W_{p}^{-1}(s) \ldots \ldots 116$

5.11 Interconnection of performance weights with plant, uncertainty description and controller. . . . . . . . . . . . . . . . . . . . 117

5.12 Weighting functions used in design template. . . . . . . . . . . . . . 121

5.13 Sensitivity function bounds defined for the benchmark controller. . . . . . . 122

5.14 Changes in $\mu$ during D-K iteration steps for the Benchmark I controller. . . 130

5.15 Singular value of Benchmark I controller and plant with Speed Model 2. . . 131

5.16 Bode plots of response of Benchmark I controller across all four channels. . 132

5.17 Pole-zero map of the Benchmark I controller (excludes 2 real poles at -242 $\mathrm{krad} / \mathrm{s}$ and $-56 \mathrm{krad} / \mathrm{s}$ and a complex zero pair at $34.6+j 34.7 \mathrm{krad} / \mathrm{s}) . \ldots 133$

5.18 Predicted local closed-loop actuator stiffness of Benchmark I controller. . . 133

$5.19 M-\Delta$ analysis $\ldots \ldots \ldots \ldots$. . . . . . . . . . . . . . . . . . . . . . . . 
$5.20 \mu$-Analysis of Benchmark I controller designed with plant uncertainty including Speed Model 2. . . . . . . . . . . . . . . . . . 136

$5.21 \mu$-Analysis of benchmark controller designed with plant uncertainty including Speed Model 1. . . . . . . . . . . . . . . . . . . . . 137

$5.22 \mu$-Analysis of 70\% stiffness anisotropy designed with plant uncertainty including Speed Model 2. . . . . . . . . . . . . . . . . . . . . 138

$5.23 \mu$-Analysis of nondiagonal performance weight $W_{p, 4}$ controller designed with plant uncertainty including Speed Model 2. . . . . . . . . . . 138

$5.24 \mu$-Analysis of Benchmark Ic1 controller designed with plant uncertainty including Speed Model 2 and CCS Model 1. . . . . . . . . . . . . . 140

$5.25 \mu$-Analysis of Benchmark Ic3 controller designed with plant uncertainty including Speed Model 2 and CCS Model 3. . . . . . . . . . . . . . 140

$5.26 \mu$-Analysis of Benchmark Ic4 controller designed with plant uncertainty including Speed Model 2 and CCS Model 4 . . . . . . . . . . . . . . . 141

$5.27 \mu$-Analysis of nondiagonal performance weight $W_{p, 4}$ controller designed with plant uncertainty including Speed Model 2 and CCS Model 3. . . . . 142

$5.28 \mu$-Analysis of nondiagonal performance weight $W_{p, 4}$ controller designed with plant uncertainty including Speed Model 2 and CCS Model 4. . . . . 142

5.29 Unbalance response case 1 for Benchmark I controller . . . . . . . . . 145

5.30 Unbalance response case 2 for Benchmark I controller . . . . . . . . . 146

5.31 Predicted damped mode shapes of rotor rigid body modes with Benchmark I controller. . . . . . . . . . . . . . . . . . . . . . . . . . . 147

5.32 Predicted damped mode shapes of rotor bending modes with Benchmark I controller. . . . . . . . . . . . . . . . . . . . . . . . . . . 148

6.1 Experimental unbalance response before and after dynamic balancing. . . . 152

6.2 Block diagram showing stimulus and response points for system transfer function measurement. . . . . . . . . . . . . . . . . . . . . . 153

6.3 Bode plots of measured loop transfer functions. . . . . . . . . . 156

6.4 Bode plots of output sensitivity functions measured at $0 \mathrm{rpm} . \ldots \ldots$

6.5 Bode plots of output sensitivity functions measured at various speeds. . . 158

6.6 Bode plots of measured complementary sensitivity function or closed-loop response. . . . . . . . . . . . . . . . . . . 159

6.7 Bode plots of measured process sensitivity function. . . . . . . . . 160

6.8 Bode plots of measured control sensitivity function. . . . . . . . . . . 161 
6.9 Rotor displacement response at 7,000 rpm following termination of blocking excitation at various CCS levels. . . . . . . . . . . . . . . . 163

6.10 Experimental stability sensitivity plot of $\log$ decrement of $\mathrm{Nc} 1$ versus destabilizing cross-coupled stiffness $Q$ for several controllers compared with the Benchmark I. . . . . . . . . . . . . . . . . . . 167

6.11 Bode plots of three controllers comparing regions of positive phase. . . . . 170

6.12 Loop gain specifications for performance and robustness trade off (The Mathworks, 2009). . . . . . . . . . . . . . . . . . 173

6.13 Maximum singular value of interaction between model uncertainty and weighted performance outputs, $\left\|T_{z v}(j \omega)\right\|_{\infty}$ or $\bar{\sigma}\left(M_{21}(j \omega)\right)$. . . . . . . . 174 


\section{List of Tables}

4.1 Summary of MBTRI experiment properties. . . . . . . . . . . . 56

4.2 The first six rotor critical speeds. . . . . . . . . . . . . . . . . 64

4.3 Support and disturbance AMB specifications. . . . . . . . . . . . 68

4.4 Linearized actuator properties for support and disturbance AMBs. . . . . . 69

4.5 Maximum static force calculated for the support and disturbance AMBs. . . 72

4.6 Dynamic characteristics of the NDE and DE AMBs. . . . . . . . . . . 74

4.7 PID controller parameters for online system identification. . . . . . . . 85

5.1 v-gap metric of uncertainty models for supercritical operation with respect to the nominal plant model. . . . . . . . . . . . . . . 108

5.2 The effect of varying CCS magnitude on the location of $\mathrm{Nc} 1$ eigenvalue. . . 112

$5.3 v$-gap metric of uncertainty models for cross-coupled stiffness variation with respect to the nominal plant model. . . . . . . . . . . . . . 112

5.4 Weighting function parameters for the benchmark controller. . . . . . . 122

5.5 Weighting function parameters for family of controllers with support stiffness anisotropy $\chi \ldots \ldots \ldots \ldots \ldots$

$5.6 \mu$-Synthesis results of controller using uncertainty models for supercritical operation. . . . . . . . . . . . . . . . . . . . . 127

$5.7 \mu$-Synthesis results of controller including CCS uncertainty $\ldots \ldots \ldots 128$

5.8 D-K iteration results for Benchmark I controller (Kdk_48_17Aug 11a). . . 130

6.1 Two-plane influence coefficient balancing results. . . . . . . . . . . 153

6.2 Gain and phase margins from first loop gain crossover of the diagonal channels using Benchmark I controller. . . . . . . . . . . . . . . . . 154

6.3 Experimental stability threshold of various controllers synthesized. . . . . 164 


\section{List of Symbols}

\section{Acronyms}

AWG American Wire Gauge

CCS Cross-coupled stiffness

Nc1 First rotor critical speed (cylindrical rigid body mode)

Nc2 Second rotor critical speed (conical rigid body mode)

Nc3 Third rotor critical speed (first rotor bending mode)

Nc5 Fifth rotor critical speed (third rotor bending mode)

OSI Onset of instability

RSSV Resonant subsynchronous vibration

\section{Greek}

$\lambda \quad$ Complex eigenvalue

$\delta_{i} \quad$ Logarithmic decrement

$\mu \quad$ Structured singular value

$\omega_{c r} \quad$ First critical speed assuming rigid bearings [ $\mathrm{rad} / \mathrm{s}$ ]

$\zeta \quad$ Damping ratio

\section{Arabic}

$\hat{c} \quad$ Optimum bearing damping [ $\mathrm{N} \mathrm{s} / \mathrm{m}]$

$I_{\mathrm{b}} \quad$ AMB bias current $[\mathrm{A}]$

$i_{\mathrm{p}} \quad$ AMB perturbation current $[\mathrm{A}]$

$K_{i} \quad$ AMB current gain [N/A]

$K_{r} \quad$ Bearing-shaft stiffness ratio 
$K_{x} \quad$ AMB negative stiffness [N/m]

$M_{m} \quad$ Shaft modal mass

$Q \quad$ Magnitude of cross-coupled stiffness $[\mathrm{N} / \mathrm{m}]$

$Q_{\max }$ Maximum allowable cross-coupled stiffness excitation [N/m] 


\section{Chapter 1}

\section{Problem Statement}

\subsection{Introduction}

Three general classes of vibration in electromechanical systems are free vibrations, forced vibrations and self-excited vibrations (Ehrich, 2004). Free vibrations are the oscillatory response of a system at its natural frequency to non-zero initial conditions alone, i.e., the homogeneous solution. Forced vibrations are produced by external forces exerting periodic action upon the system leading to a response at the frequency of the excitation. The causative force is completely independent of the resulting vibration. Residual imbalance is a common source of synchronous vibration in rotating systems, and an example of a forced vibration. Self-excited vibrations are produced by oscillating forces that are the product of the oscillatory motion of the system itself (Vance et al., 2010). The term self-excited is perhaps misleading as the system does not spontaneously vibrate on its own. Rather, a positive feedback interaction with its environment causes energy to be transferred into a natural frequency of the system. This interaction is not evident from an analysis of the governing equations of motion, hence the term self-excited (Paidoussis et al., 2011). In fluid compressors positive feedback mechanisms produced by dynamic interactions between the compressed fluid and components in the flow path can lead to self-excited vibration of the 
rotor. The fluid-structure interaction transfers rotational energy from the working fluid to rotor modes. The rotor mode excited is usually below the running speed leading to the term resonant subsynchronous vibration or RSSV (Fozi, 1987). If these forcing mechanisms overcome the damping provided by the support bearings the result is unbounded vibration or rotordynamic instability (Kwanka, 2000). This dissertation considers exclusively the effects of rotordynamic instability in centrifugal compressors, which are an integral part of chemical process, and oil and gas industries. A photograph of a multistage compressor is shown in Figure 1.1. The upper casing has been removed to expose the rotor, six centrifugal impellers and the flow path within the machine. Typical rotating speeds of these turbomachines range from 3,000 to 20,000 rpm and they may be driven by electric motors, gas turbine or steam turbine engines. A common source of excitation leading to rotordynamic instability occurs in the vicinity of the seals and impellers. Seals are required at multiple locations within the compressor to prevent the leakage of fluid from high pressure to low pressure areas, while the impellers impart kinetic energy to the fluid (Brown, 2005). The design of these components may be such that significant reaction forces are generated on the rotor normal to rotor displacement. These forces are termed aerodynamic cross-coupled stiffness (CCS) due to their origin in the fluid flow path of the compressor (Childs, 1993).

Experimental data on the fluid-structure interaction within the rotor-stator clearances is difficult to obtain. As a result, there is heavy reliance on semi-empirical models to predict the magnitude of aerodynamic excitation. These models depend on parameters such as the mechanical power output of the compressor, component dimensions, the ratio of discharge and suction gas densities, as well as experiences with installed machines. With this empirical knowledge, considerable uncertainty remains in these predictions, leading to conservative designs at best, or costly retrofits once problems arise in the field. Field operating conditions that vary over time, as well as the prohibitively high cost of constructing a complete prototype compressor add to the challenges of developing widely applicable solutions (Vance et al., 2010). In a recent benchmark study comparing analytical seal 


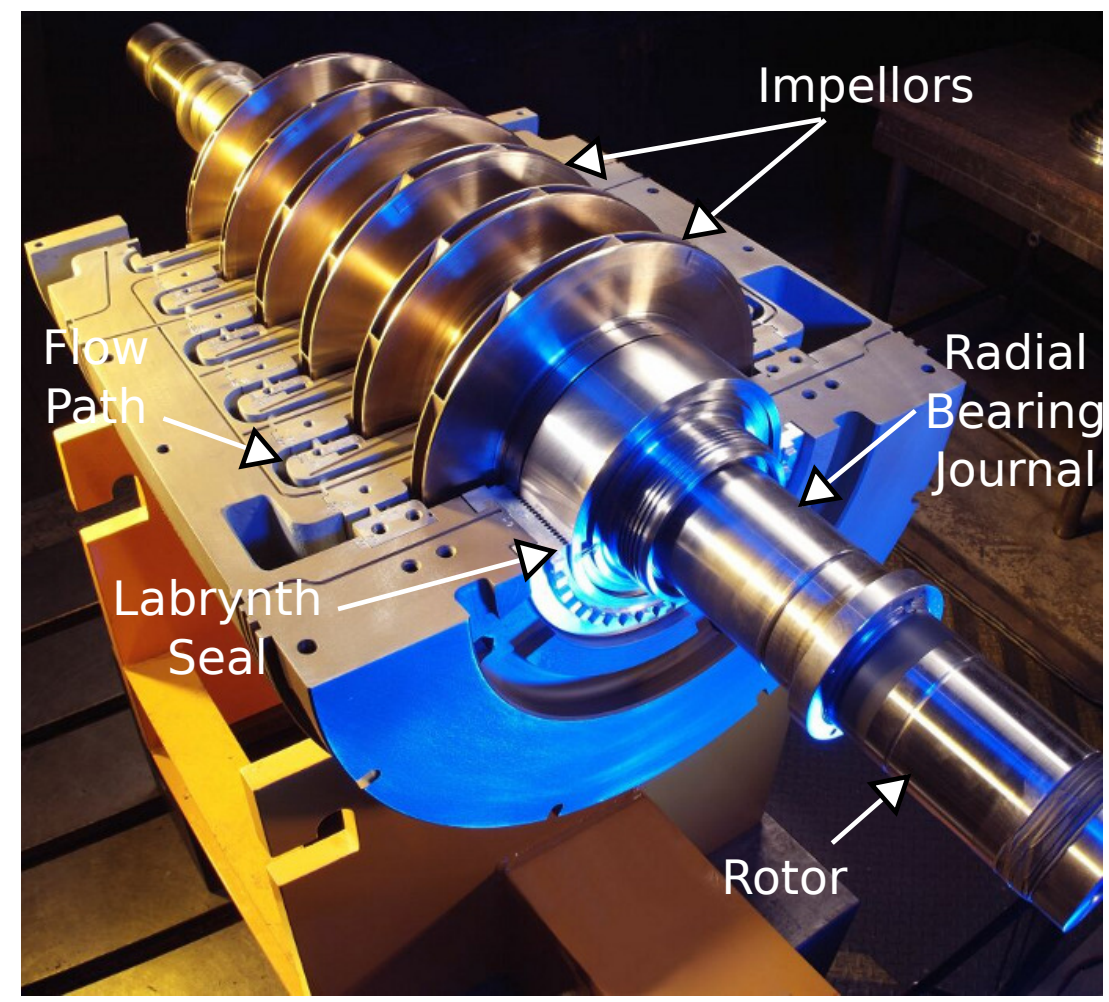

Figure 1.1: A multistage centrifugal compressor with upper casing removed showing the major components (Bidaut \& Baumann, 2010).

rotordynamic coefficients computed by twenty independent respondents from industry and academia, Kocur et al. (2007) noted two orders of magnitude variation in principle stiffness and three orders of magnitude variation in cross-coupled damping. The resulting widely differing stability predictions from the respondents are indicative of the significant uncertainty present in current models. The use of computational fluid dynamic (CFD) analysis to improve the understanding of fluid-structure interactions is growing. However, the tools in their current form are not mature for use at the design stage (API 684, 2005).

Increased operating efficiency, higher discharge pressure ratios and reduced package size are driving a trend towards slender and higher speed rotor designs in turbomachine applications. This is a direct result of the reduced torque required for the same mechanical power output, to turn a lighter (flexible) rotor at a higher speed than a heavier (rigid) rotor at a lower speed (Hetherington et al., 1990). With centrifugal compressors, in particular, the demand for higher rotational speeds and pressures requires multiple impeller stages 


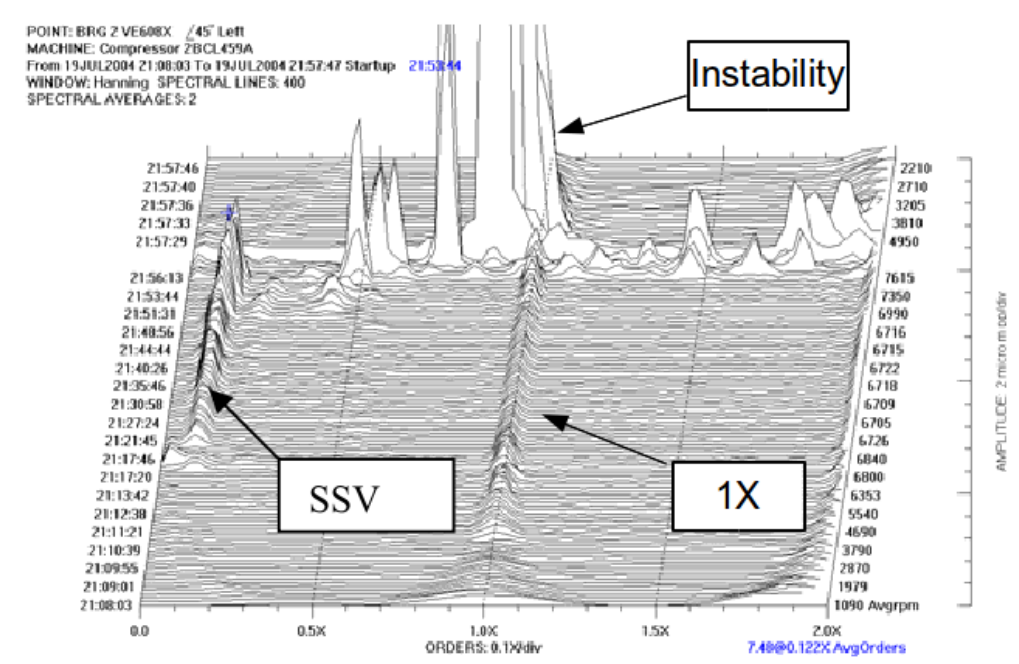

Figure 1.2: A waterfall plot of vibration spectra during startup of a high pressure centrifugal compressor showing the subsynchronous vibrations (RSSV) leading to instability (Moore et al., 2006).

(and interstage seals) which leads to longer, more flexible rotors which are more prone to aerodynamic instability (Barrett, 1979; Moore et al., 2006). The primary design issue with high-pressure compressors is the trade-off between rotordynamic stability and thermodynamic performance (Baumann, 1999). A rotor optimized for rotordynamic stability, i.e., heavy and rigid, tends to have poor aerodynamic performance. Higher pressure machines require aptly sized balance piston seals to partly offset the aerodynamic thrust load. The destabilizing influence of the balance piston on rotordynamics increases with pressure (Moore \& Ransom, 2010). Such concerns may impact project schedules (by requiring full load, full speed tests for new designs), reduce production rates (by restricting operating speeds) and cause machine and plant damage in the event of instability. Figure 1.2 shows vibration spectra of a compressor demonstrating instability during startup as a result of CCS forces at the fluid seals leading to increasing RSSV. One can distinguish between the synchronous response to unbalance (1X line) and the RSSV at $113 \mathrm{~Hz}$ (approx 0.1X) which begin as the rotor reaches $6,800 \mathrm{rpm}$, indicating the dependence of the CCS force magnitude on the operating parameters of the compression system. The chaotic vibration marked by the "instability" arrow indicates likely rotor-stator rubbing and perhaps damage. 
Fluid film bearings, the standard passive mechanical bearing for high speed rotating machinery, have fixed stiffness and damping characteristics related to their physical construction and lubricant properties (Dimond, 2011). Fluid film bearings, particularly fixed geometry journal bearing designs, have high cross-coupled stiffness coefficients which can lead to rotordynamic instability under certain conditions. The introduction of tilting pad journal bearings provided a solution with higher damping and reduced destabilizing tendencies. However, tilting pad designs have been unable to keep pace with evolving high performance compressor designs (Criqui \& Wendt, 1980). Faced with rotordynamic instability in a machine with passive bearings, the only alternative is a physical redesign of the rotor, bearings and/or seals to improve the overall damping characteristics. This highlights a major advantage for the application of active magnetic bearing (AMB) technology - the ability to modify the control algorithms at any time to provide optimal damping to stabilize rotor behavior (Habermann \& Brunet, 1984). The advantages of implementing an AMB system include, but are not limited to: complete elimination of oil-based lubrication systems, low parasitic power loss, direct control of the rotordynamics, lower maintenance costs and longer system life (Schweitzer \& Maslen, 2009). For these reasons, the use of AMBs in turbomachinery applications for oil and gas production provides clear technical and economic advantages over fluid film bearings (Ahrens et al., 2000). AMB technology paves a path to introduce active vibration control to the problem of rotordynamic instability. In this application, the potential benefits of feedback are the enabling of optimal performance of the compression system as the operating conditions change over the life of the machine, and a reduction in the effects of disturbances on the system (Doyle et al., 1990). Model-based robust control algorithms such as $\mu$-synthesis have received recent attention as a powerful framework for the design and analysis of practical multivariable controllers to stabilize flexible rotors with uncertain plant dynamics and constrained actuator bandwidth (Schonhoff, 2002; Maslen \& Sawicki, 2007). $\mu$-synthesis is the only mature robust multivariable control design method with a well developed theory and matching 
software tools (Steinbuch et al., 1998).

\subsection{Objectives}

The literature is lacking an investigation of active control in the presence of uncertain aerodynamic loads on flexible rotors supported by AMBs. In particular, an investigation that focuses on both the control theory and rotordynamics unique to the problem. Prior research has focused on subcritical rigid rotors while the centrifugal compressor designs most susceptible to this instability tend not to be of this construction (Smith, 1995; Lang et al., 1996; Wurmsdobler, 1997). The objectives of this study are to:

1. Commission an AMB test rig that will allow for the study of active control of rotordynamic instability. The instability may be the result of arbitrary static and dynamic loads representing the expected aerodynamic loads from seals and impellers. The test rig will exhibit rotordynamic characteristics similar to those of an industrial centrifugal compressor while retaining sufficient simplicity to be operated within the Rotating Machinery and Controls Laboratory at the University of Virginia. The test rig will allow operation over a speed range that includes one lateral bending natural frequency.

2. Investigate and evaluate model-based control algorithms based on their ability to maintain system stability and performance with respect to the uncertain aerodynamic loads.

3. Develop uncertainty models with minimal conservatism to capture variations in the dynamics of rotor-bearing system.

4. Compare the stability and performance predictions to experimental stability measurements. 
5. Investigate effects of such as actuator bandwidth and slew rate limits on achievable performance.

\subsection{Contributions}

The major contributions of this dissertation are to:

1. Document the development of a versatile and realistic experimental testbed for research on control system applications.

2. Provide the first demonstration of rotordynamic instability in a supercritical rotor supported on active magnetic bearings.

3. Design and implement robust control algorithms able to increase the rotordynamic instability threshold of the rotor-AMB system.

4. Define the limitations imposed by uncertainty and actuator dynamics on achievable performance of control algorithms designed.

5. Harmonize mechanical vibration and control systems concepts to develop practical guidelines for researchers, and machine designers and operators considering the design, construction, modeling and evaluation of supercritical machines on magnetic bearings.

\subsection{Outline of the Dissertation}

In addition to the introductory Chapter 1 , and Chapter 7 which presents the concluding remarks, the main body of the dissertation is developed in five chapters, Chapters 2 through 6, and three appendices, Appendices A, B and C. Chapter 2 presents a review of pertinent literature on rotordynamic instability, and active control of vibration with a focus on magnetic bearing applications involving rotating machinery. Chapter 3 discusses the mechanics 
of rotordynamic instability as well as fundamental limitations on achievable performance using feedback control. Chapter 4 describes the design, construction, modeling and validation of the MBTRI test rig. Chapter 5 presents the $\mu$-synthesis design approach and discusses the selection of frequency domain performance weights and the development of uncertainty models required to synthesize robust controllers. The uncertainty models are compared using the gap metric approach. Whilst $\mu$-analysis of the synthesized controllers is used to quantify their robustness properties. Chapter 6 contains experimental data concerning the operation of the rotor above its bending natural frequency, and the measurement of the stability threshold with respect to rotordynamic instability due to CCS. A discussion of these results within the context of the rotordynamics, i.e., existence of optimal damping and fundamental limitations on feedback controller performance. Chapter 7 concludes the dissertation with a summary of the work completed and a discourse on further research directions. 


\section{Chapter 2}

\section{Literature Review}

\subsection{Rotordynamic Instability}

Rotordynamic instability is marked by the sudden onset of vibrations at a rotor natural frequency below the running speed of the machine. The amplitude of this vibration may increase with running speed and discharge pressures to the extent that safe operation of the machine at higher power levels is not possible due to the risk of failure of the rotor from the alternating flexural stresses (Ehrich \& Childs, 1984). Three well publicized examples of rotordynamic instability were the 1971 "Kaybob" case involving a nine-stage natural gas centrifugal compressor experiencing violent RSSV during testing that defied numerous hardware modifications, the 1974 "Ekofisk" case involving an eight-stage offshore gas re-injection compressor displaying similar RSSV instability, and the 1976 problem involving the rocket-exhaust powered high-pressure fuel and high-pressure oxygen turbopumps for the now retired Space Shuttle main engine. In all examples, the high performance demanded of these turbomachines exceeded the capabilities of analytical and computational tools of the day to determine rotordynamic stability. While solutions were eventually found for all three incidents, the search consumed considerable time and expense. In the case of both problem compressors, the precise source of instability was never determined (Vance 
et al., 2010). More recent cases of instability in high pressure centrifugal compressors continue to highlight the major potential destabilizing influence of seals and point to a need for deeper understanding of the effects of seal dynamics on system stability (Moore et al., 2006).

The seminal work of Alford (1965) offered the first quantitative analysis of aerodynamic mechanisms leading to rotordynamic instability in jet engine axial flow compressors and turbines. Alford highlighted two sources of self-excited rotor vibrations: the circumferential variation in the static pressure distribution in the labyrinth seals, and circumferential variation of torque on the impeller due to eccentric rotor orbits. In the vicinity of the seals, the rotor displacement amplitude was known to be directly proportional to the amplitude of pressure oscillations within the seal. The phase angle between the two rotating vectors varied with the flow characteristics and speed of rotation, i.e., the engine aerodynamic load. At particular phase angles the vectors summed to encourage whirl in the direction of rotation. Concentric location of an impeller within the allowed clearance produces balanced forces and torques on the impeller and hence the rotor. On the other hand, eccentric rotor orbits relative to the stationary components in the clearance lead to variations in the aerodynamic efficiency across the circumference of the impeller. The impeller efficiency is related to its ability to accelerate the fluid. Therefore, varying impeller clearance results in an unbalanced torque on the rotor as a function of position. As with the aforementioned seal pressure variation, certain operating conditions may cause the rotating torque vector to act in a manner conducive to forward whirl, once again leading to a self-excited positive feedback phenomenon. In both cases involving the seal and impeller, rotational energy from the working fluid is coupled into a rotor natural frequency by an aerodynamic mechanism, leading to the term aerodynamic cross-coupling (Ehrich, 2004).

Bulk flow analysis is the name given to a family of analytical approaches for simplifying the complex fluid dynamics occurring within seal designs (Childs, 1993). Modeling seals using computational fluid dynamics (CFD) is capable of capturing more details on 
the fluid-structure interactions and are an improvement over the bulk flow models. However, significant uncertainty is still documented in predictions of dynamic seal coefficients highlighting the need for experiments to improve our knowledge of the underlying physics (Kocur et al., 2007; Wagner et al., 2009).

Whereas Alford predicted the CCS as a function of stage torque and dimensions, Wachel \& von Nimitz (1981) refined his empirical model for aerodynamic cross-coupling to include additional parameters such as density and molecular weight of the fluid being compressed. The cross-coupled stiffness $(\mathrm{N} / \mathrm{m})$ at each impeller stage is

$$
Q=\frac{16 \times P \times \mathrm{MW}}{D \times h \times f} \times \frac{\rho_{D}}{\rho_{S}},
$$

where MW is the gas molecular weight, $D$ is impeller diameter $(\mathrm{m}), h$ is the restrictive dimension in the flow path $(\mathrm{m}), f$ is the rotation speed $(\mathrm{Hz}), P$ is compressor mechanical power $(\mathrm{kW}), \rho_{\mathrm{D}}$ and $\rho_{\mathrm{S}}$ are the compressor discharge and suction densities $\left(\mathrm{kg} / \mathrm{m}^{3}\right)$, respectively. Within the last decade, the industry has accepted a modified Alford's equation which replaces the MW with a constant of 30 to better match the database of experience built up from the installed base of centrifugal compressors (API 684, 2005). While these empirical guidelines are easy to apply, they remain subject to significant uncertainty, hence the use of full-load, full-pressure tests on new compressors designs prior to delivery (Vance et al., 2010). Such tests are an expensive option for the customer, but at times are required to increase confidence in as yet unproven compressor designs.

The term cross-coupled stiffness (CCS) arises from observations of a force $F_{\mathrm{xc}}$ acting on the rotor with a line of action that is perpendicular to the rotor displacement. Mathematically

$$
F_{\mathrm{xc}}=\left[\begin{array}{c}
f_{x} \\
f_{y}
\end{array}\right]=Q\left[\begin{array}{cc}
0 & 1 \\
-1 & 0
\end{array}\right]\left[\begin{array}{l}
q_{x} \\
q_{y}
\end{array}\right],
$$

where $f_{x}, f_{y}$ and $q_{x}, q_{y}$ are forces and displacements along the $x$ and $y$ axes respectively, and 
$Q$ is the magnitude of the cross-coupled stiffness. As $Q$ increases there is a tendency for a forward whirl (precession of the rotor orbit at a frequency equal to rotor's lowest natural frequency) to be excited. This in turn counteracts the restorative damping force produced largely by the support bearings, and can lead to unbounded oscillations at a rotor natural frequency below the running speed.

Rotordynamic instability is not limited to compressors and their associated fluid-structure interactions. Large induction motors with eccentric rotor orbits are known to generate RSSV through electromechanical interactions between the stator and rotor known as unbalanced magnetic pull (Holopainen, 2004).

\subsection{Rotor Vibration Control using AMBs}

\subsubsection{General}

Rotors may be loosely characterized as being either rigid or flexible (elastic) designs. Rigid rotors have their operating speed far enough below their lateral bending natural frequency that this frequency is not easily excited under normal operation. Flexible rotors have one or more lateral bending natural frequencies within the operating speed range or control bandwidth. These modes are lightly damped and when the system dynamics are viewed in the Argand plane, their eigenvalues are clustered close to the imaginary axis. As a result, the control of flexible rotors presents a modeling and identification challenge for the lumped parameter approaches favored in linear control system design are approximations of the complex distributed parameter dynamics that govern the behavior of the system (Balas \& Doyle, 1994). Early theory and experimental work on robust control for flexible structures with uncertain dynamics focused on non-rotating large flexible space structures (Balas, 1990). The reduced weight of these structures is an important advantage for space-borne applications. However, as with rotors, structural flexibility introduces new challenges in the modeling and identification of several closely spaced, lightly damped resonant modes 
and the issue of sensor/actuator location (Balas \& Young, 1999). Flexible space structures possess lower natural frequencies than rotor-AMB systems and do not exhibit gyroscopic behavior. Nevertheless, the theory and practice of the model-based control paradigm developed for active control of these structures remain fully applicable to AMB systems. From the control standpoint the AMB provides a benchmark problem for numerous fields of control design and analysis. This has led to rich body of research on the use of AMBs for active vibration control using just about every algorithm from classical (PID and lead/lag) to nonlinear and adaptive feedback methods (Schweitzer \& Maslen, 2009)

The tools of choice to solve the practical robust control requirements of elastic rotors on AMBs are $H_{\infty}$ optimal control, linear fractional transformations (LFTs), and the structured singular value $\mu$ (Doyle, 1982; Doyle et al., 1991; Packard \& Doyle, 1993). $H_{\infty}$ optimization theory serves as the foundation for constructing robust controllers that minimize the worst case gain from disturbance inputs to weighted performance outputs for the nominal plant dynamics. This is a convex optimization problem, and state space solutions for a tight upper bound may be computed by solving two algebraic Riccati equations (Zhou et al., 1996). LFTs provide an attractive means to systematically incorporate uncertainty into models used for synthesis and analysis.

The $\mu$-analysis and synthesis frameworks use LFTs to extend $H_{\infty}$ optimization to provide a practical means of handling model uncertainty to meet robust performance specifications in multivariable systems. The solution to the robust performance problem through the computation of $\mu$ is a nonpolynomial (NP) time problem, leaving the calculation of tight upper and lower bounds as the only path to a satisfactory solution (Braatz et al., 1993). Finding the $\mu$ upper bound for the complex $\mu$-synthesis problem is a nonconvex problem, but can be approximated by a two step iterative procedure called D-K iteration. The individual steps are convex and involve $H_{\infty}$ optimization. While convergence to a global optimum is not guaranteed (owing to the lack of joint convexity of each step), satisfactory solutions are often found for practical engineering problems (Stein \& Doyle, 1991). The 
complex $\mu$-synthesis solution described may, in some cases, be conservative since modeled uncertainties are always treated as perturbations in a complex plane. Since many practical problems involve real-valued uncertainties, the use of mixed $\mu$-synthesis algorithms may yield performance gains (Young, 1996). However, the gains come with the cost of increased computational complexity of the D,G-K iterative algorithm used to compute tight $\mu$ bounds.

Linear matrix inequalities (LMIs) are useful in formulating and solving numerous convex problems in systems and control theory (Boyd et al., 1994), and are an alternative to the algebraic Riccati solution for $H_{\infty}$ optimization. The work of Scherer et al. (1997) popularized multi-objective (or mixed-objective) dynamic output feedback using LMI optimization. The LMI framework provides the ability to include constraints on input/output values, closed-loop pole locations, $H_{2}$ (stochastic) performance as well as $H_{\infty}$ (robustness) objectives. Rather than translating all these objectives (often imperfectly) into frequency domain weights as is done in the mixed-sensitivity framework, the objectives are represented as LMIs in their native form. Using a single Lyapunov function for all the objectives ensures the problem is convex. Though somewhat conservative, this approach often yields a mathematically tractable single LMI optimization problem which can represent a physical system to be controlled with multiple norm and other closed-loop specifications. Incorporating robust pole placement techniques such as those described by Chilali et al. (1999) may be useful in enhancing the damping of rotor modes destabilized by the CCS. The computational burden of solving problems involving plants of relatively high order is greater for LMI methods than the two algebraic Riccati equation approach typically used for the $H_{\infty}$ optimization steps in D-K iteration.

\subsubsection{Rotating Machinery Applications}

Early use of AMBs as sources of excitation for rotor systems was reported on during the First International Symposium on Magnetic Bearings by Ulbrich (1988). In this work, a 
single radial AMB was placed at the midspan of a rotor supported by rolling element or journal bearings. The actuator was used to generate nonconservative forces, e.g., CCS, or standard test forces, e.g., impulse or step input, to evaluate system damping.

The complete removal of hydrodynamic bearings from the system and the exclusive use of the AMBs to investigate rotordynamic instability and the potentials using AMBs for active control of instability are an open area of research. Smith (1995) reported on aerodynamic loading of a hypothetical centrifugal compressor, and presented simulation results on the use of gain-scheduled Kalman filters and linear quadratic control laws. The aim of this work was to determine performance of optimal control algorithms versus local PID controllers. Modeling of the seal and impeller characteristics producing the instability was performed in detail and reaffirming numerical simulation results were obtained for a nominal plant model. For instance, using a Kalman filter permitted a $40 \%$ increase in the aerodynamic excitation level prior to performance degradation. However, the tolerance for modeling error, a known weakness of linear quadratic Gaussian control, was poor and no stability robustness or experimental validation was performed.

The application of adaptive control algorithms to detect and compensate for unknown aerodynamic CCS was first reported on a rigid rotor by a group at the Vienna University of Technology (Wurmsdobler et al., 1996; Wurmsdobler, 1997). In these works, the recursive prediction error method was used to estimate the states of the plant and parameters of a Kalman observer. With the application of a pole placement objective, a suitable controller was subsequently synthesized using the plant estimates. In simulation, the algorithm demonstrated the ability to track changes in the CCS magnitude and update control gains to damp the subsequent oscillations. For a rigid rotor-AMB plant represented by a dynamic model with an eight element state vector, four inputs and four outputs, a total of 96 parameters were estimated online. The large number of parameters to be estimated led to conclusions that numerical complexity and computational demands may complicate practical implementation of the algorithm. In an attempt to reduce the computational burden, other 
researchers substituted the pole placement design for LQR synthesis (Lang et al., 1996). While this simpler algorithm was more amenable to practical implementation, it yielded a reduced stability margin with respect to the magnitude of CCS than the pole placement design. Later experimental work combined the adaptive CCS compensation algorithm with a feedforward open-loop control to reduce the synchronous vibrations due to mass unbalance (Hirschmanner et al., 2002).

More recently, AMBs have become a favored tool for the evaluation of rotordynamic parameters for hydrodynamic bearings as well as seals (Kwanka, 2000; Nordmann, 2009). This unique advantage stems from the ability of AMBs to simultaneously serve as a rotor support, a source of dynamic excitation and a force measurement tool for the rotordynamic system. In an extensive study on the stability of rotor systems, Cloud (2007) used AMBs to generate cross-coupled stiffness and also harmonic excitation. The test rotor in his study was supported by tilting pad journal bearings and the goal was to evaluate several damping ratio estimation algorithms. The damping ratio is an indicator of the stability of a system. As part of a combined numerical and experimental study aimed at improving predictions of the rotordynamic coefficients of labyrinth seals, Wagner et al. (2009) constructed a full scale magnetic bearing test rig to support and perturb a rotor containing a test seal at midspan. The test rig operated to a maximum speed of 15,000 rpm, and had a test pressure limit of 70 bar. The AMB provided the means to dynamically excite the seal up to $400 \mathrm{~Hz}$ with both forward and backward whirl orbits. Using pressure measurements from the test seal as well as dynamic force measurements from the AMBs, experimental seal rotordynamic coefficients were obtained and compared with numerical analysis by CFD. Compressor manufacturers are beginning to use magnetic exciters temporarily attached to one end of a rotor during full-load testing to obtain unobtrusive measurements of the rotordynamics prior to customer delivery (Moore et al., 2002).

The University of Virginia's Rotating and Machinery Controls Laboratory has played a significant role in the development of prototype machines and control algorithms for AMB 
systems over the preceding three decades, some notable developments will be highlighted. The use of AMBs in a variety of practical applications from industrial canned pumps for caustic products to continuous flow artificial heart pumps was pioneered by Allaire et al. (1989, 1996). Yates \& Williams (1988); Williams et al. $(1990,1994)$ presented novel implementations of digital controllers with fault-tolerant capabilities for use with rotor-AMB systems. In Knospe et al. $(1995,1997)$ new results were presented on the use of adaptive open-loop methods to achieve robust minimization of the rotor unbalance response over a range of speeds in subcritical rotors. Noh (1996) worked on techniques to improve the self-sensing of the rotor position using information gleaned from the current feedback waveform of the switching power amplifier. Chatter, a self-excited vibration resulting from the interaction between an oscillating machine tool and a work piece, is an issue for high speed milling machines. In a prototype $67 \mathrm{~kW} 32,000 \mathrm{rpm}$ milling spindle supported by AMBs, $\mu$-synthesis control was used to minimize the compliance of the tool tip and thus reduce the chatter phenomenon (Stephens, 1995; Fittro, 1998; Chen \& Knospe, 2007). Robust multivariable control was applied to an energy storage flywheel rotor with structural flexibility to enable operation through the first bending natural frequency ( $\mathrm{Li}, 2006)$. Compressor surge is a destructive low frequency resonance produced by unstable flow in the compression system. A technique using a thrust AMB to modulate the tip clearance of a single unshrouded impeller in a centrifugal compressor thus actively controlling surge was proposed by Sanadgol (2006), and demonstrated successfully by Yoon et al. (2010). 


\section{Chapter 3}

\section{Rotordynamic Instability}

This chapter analyzes the fundamental mechanics and system theory of rotordynamic instability. From the viewpoint of mechanical vibrations, the self-excited instability of rotating systems presents a dynamically rich problem. Since the 1970s, solutions developed following cases of rotordynamic instability have resulted in analytical (Barrett et al., 1978) and empirical (Wachel \& von Nimitz, 1981) tools to improve the prediction of the onset of instability and/or re-design rotor-bearing system to attain close to optimal support damping. Active control using magnetic bearings holds promise of further improvements. However, several limitations do exist. Classical control systems theory provides vital insight to implicit limitations governing the achievable performance of any feedback controller. Feedback control is an interdisciplinary field and harmonizing the contributions from mechanics and systems theory is paramount in motivating new techniques to delay the onset of instability and understanding any limitations (Goodwin et al., 2000). 


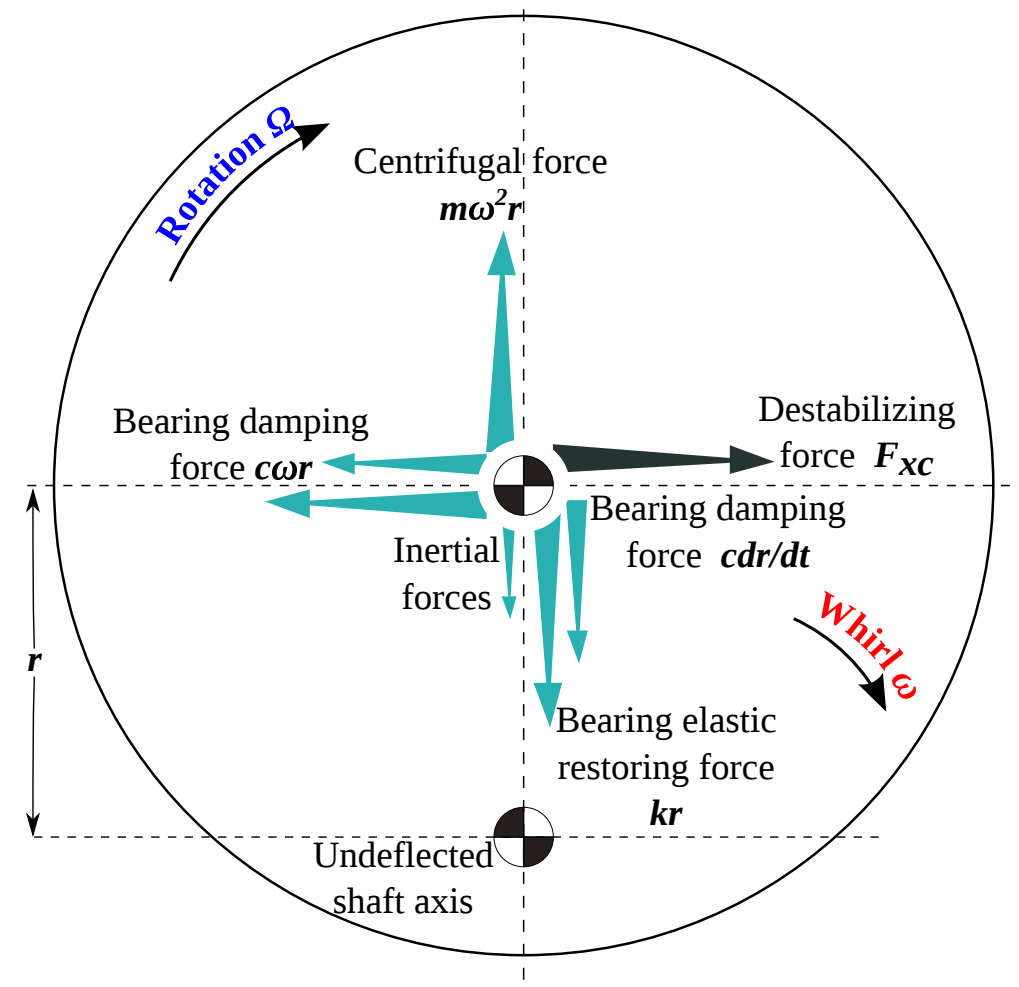

Figure 3.1: The force balance for a whirling shaft (Ehrich, 2004).

\subsection{A Mechanical Systems Perspective}

\subsubsection{Whirl Instability}

Rotordynamic instability is marked by asynchronous whirl at a frequency other than rotating speed $\Omega$ of the rotor. Whirl is the precession of the rotor orbit at a natural frequency of the rotor and denoted as either forward or backward whirl relative to the direction of rotation of the rotor (Ehrich, 2004). Asynchronous whirl arising from self-excited vibration is more destructive than synchronous whirl arising from rotor imbalance because of the buildup of stress within the rotor (Childs, 1993). Forward whirl is predominantly excited by both instability and imbalance. During whirl the destabilizing mechanism produces a tangential force $F_{\mathrm{xc}}$ on the rotor in response to a deflection $r$ of the rotor from its centerline. A force balance analysis of the radial and tangential forces acting in the rotor-bearing system is necessary to explain the dynamics of the instability (see Figure 3.1). The net tan- 
gential force encourages the rotor to whirl in the direction of rotation and is counteracted by other forces in the system. The most important of the restorative forces is the bearing damping, which irreversibly dissipates vibrational kinetic energy of the rotor. Above a particular rotor speed, $\Omega$, the tangential force may exceed the effective system damping and the rotor will whirl. The whirling motion increases the deflection of the rotor, which further increases the amplitude of the tangential destabilizing force leading to the self-excitation phenomenon. Considering an ideal rotor-bearing system represented by a lumped mass $m$, mounted on bearings with stiffness $k$ and damping $c$, undergoing a deflection $r$, the radial force balance when whirling at an angular frequency of $\omega \mathrm{rad} / \mathrm{s}$ is

$$
m \omega^{2} r=m \frac{d^{2} r}{d t^{2}}+c \frac{d r}{d t}+k r
$$

The left hand side of the above equation is the centrifugal force on the rotor, and the right hand side is the sum of the rotor inertia, bearing damping and elastic restoring forces. The cross-coupled stiffness mechanism results in a destabilizing force $F_{\mathrm{xc}}$ which is a function of the rotor radial deflection, $r$. This force was parametrized in Equation (2.2), hence the relationship with $r$ is of direct proportionality, i.e., $F_{\mathrm{xc}}=Q r$. The tangential force balance is

$$
2 m \omega \frac{d r}{d t}+c \omega r=F_{\mathrm{xc}}
$$

where the left hand side is the sum of the rotor inertial and bearing damping forces. The solution to the above system of differential equations has the form $r=r_{0} e^{(a+j b) t}$, where sign of $a$ reflects the system stability, and $r_{0}$ the initial vibration amplitude. If $a<0$, whirl amplitudes will decay exponentially with time, and if $a>0$, whirl amplitudes will increase with time. Operating conditions that produce the solution $a=0^{+}$, define the onset of instability (OSI). The condition for stability of the system may be expressed as

$$
a=\frac{Q-c \omega}{2 m \omega}<0,
$$


and restated in dimensionless form as

$$
\frac{Q}{\omega^{2} m} \leq 2 \frac{c}{\omega m} .
$$

This last equation reveals important properties of the self-excited vibration namely:

1. Since $Q$ is typically a function of rotating speed, as $\Omega$ increases the left-hand side may surpass the right-hand side leading to instability, i.e., $a>0$.

2. Increasing the level of system damping $c$ results in the OSI occurring at a higher speed.

3. The whirl frequency as the system instability is independent of the destabilizing force and is equal to

$$
\omega=\sqrt{\frac{k}{m}},
$$

which coincides with the first natural frequency of the rotor-bearing system, $\mathrm{Nc1}$.

Linear analysis of the system at OSI predicts an exponential growth in whirl orbit amplitudes once the stabilizing damping forces have been overcome (see Figure 3.2a). In actuality, unstable whirl motion in rotating machinery does not follow this exponential growth pattern. Large amplitude oscillations allow nonlinear mechanisms to dominate the motion and dissipate more energy than predicted by the linear model (Tondl, 1991; Ehrich, 2004). The result is a steady state limit cycle orbit, which increases with magnitude of the destabilizing force (see Figure 3.2b). The physical dimensions of the clearance between the rotor and stator components serves as the ultimate limit of the orbit amplitude, and once these limits are reached serious damage to the rotor and stator components occurs.

The plot in Figure 3.3 illustrates the vibration frequency spectrum of a rotor approaching the onset of instability. While rotating at 16,000 rpm, the presence of midspan crosscoupled stiffness of $1600 \mathrm{~N} / \mathrm{mm}$ triggers forward whirl at the first critical speed, Nc1. However, the restorative damping forces of the bearings have limited the whirl amplitude 

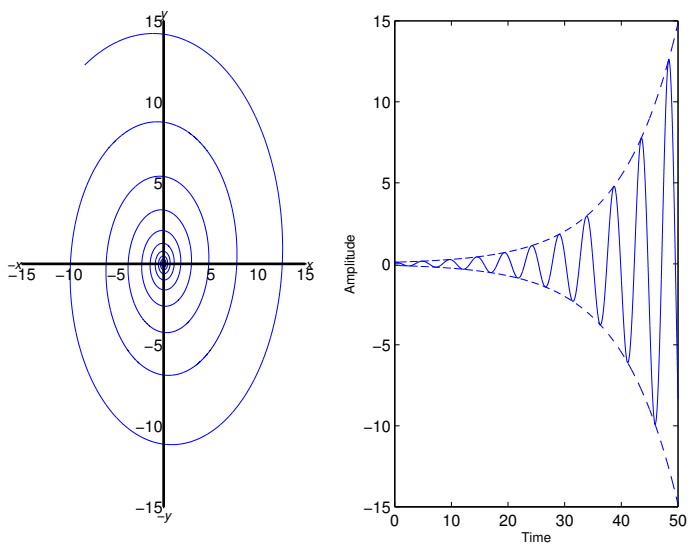

(a) Linear system model.
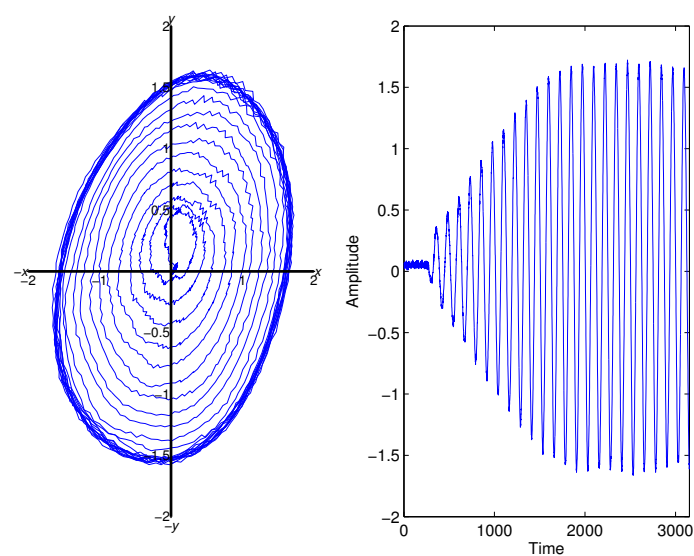

(b) Nonlinear system model.

Figure 3.2: Predicted shaft motion at the onset of instability.

to a quarter of the synchronous response. Increasing the magnitude of CCS serves to increase the whirl amplitude at $\mathrm{Nc1}$ and push the rotor-bearing system closer to the point of instability. The waterfall plot is used to compare the vibration frequency spectra of a rotorbearing system as the rotating speed is varied. Figure 3.4a shows the vibration spectra in the absence of CCS. The plot is dominated by the synchronous unbalance response, which peaks as the rotor crosses three critical speeds before reaching 18,000 rpm. Figure 3.4b shows the vibration spectra in the presence of a constant $1800 \mathrm{~N} / \mathrm{mm}$ CCS up to 16,000 rpm. Across the operating speed range, Nc1 has been solely excited by the CCS. This plot differs slightly from the behavior of practical compressors encountering instability where the fluid-induced CCS is a function of operating speed and other parameters. In this plot, a constant destabilizing CCS force is generated by a magnetic actuator.

\subsubsection{Experimental Stability Measurement}

This dissertation is concerned with determining the conditions under which a rotor-bearing system is either stable or unstable and not with the behavior following the onset of instability, which was shown earlier to enter a nonlinear regime. Accordingly, stability is 


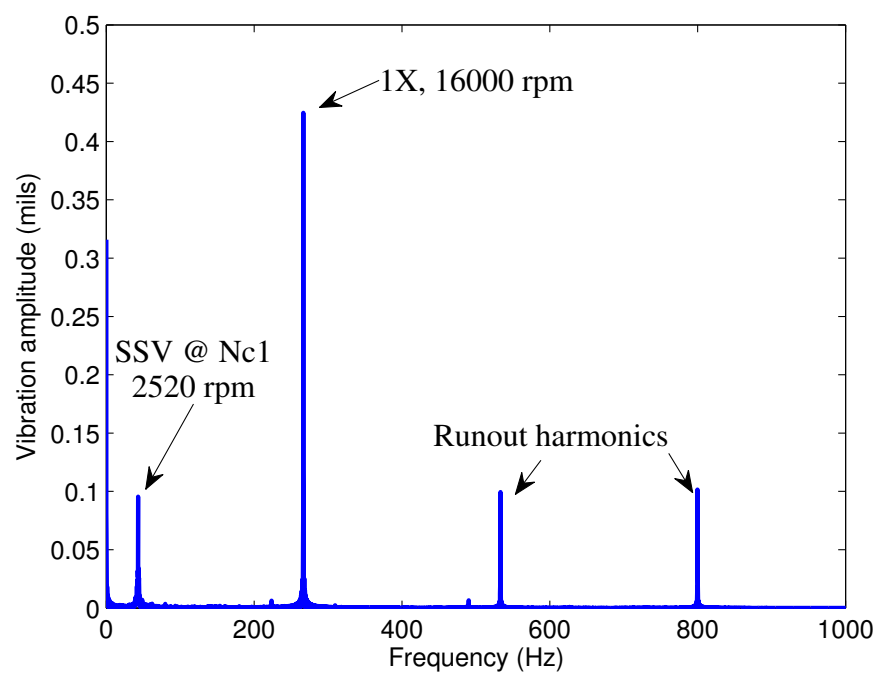

Figure 3.3: Vibration amplitude spectrum of magnetic bearing supported flexible rotor operating at 16,000 rpm. A CCS of magnitude $1600 \mathrm{~N} / \mathrm{mm}$ is acting at the midspan leading to a SSV at $2250 \mathrm{rpm}$.

considered in the exponential sense, i.e., the minimum rate of decay of vibration is specified by the real part of the system eigenvalues. This is a special case of asymptotic stability as linear models of the rotor-bearing system are used for analysis (Chen, 1998). Stability analysis involves the computation or measurement of the complex eigenvalues $\lambda_{i}$ of the rotor-bearing system, each of which may be represented by the expression

$$
\lambda_{i}=-\zeta_{i} \omega_{i, n} \pm j \omega_{i, n} \sqrt{1-\zeta_{i}^{2}}
$$

where $\zeta_{i}$ is the damping ratio, and $\omega_{i, n}$ is the undamped natural frequency of the $i$-th mode (Inman, 2006). A negative real part of the eigenvalue, i.e., $\zeta_{i}>0$, denotes stability of the associated vibration mode. In the machine vibration community the logarithmic decrement $\delta_{i}($ or log dec) is commonly used as a parameter for stability of a mode

$$
\delta_{i}=-2 \pi \frac{\operatorname{Real}\left(\lambda_{i}\right)}{\left|\operatorname{Imag}\left(\lambda_{i}\right)\right|}=2 \pi \frac{\zeta_{i}}{\sqrt{\left(1-\zeta_{i}^{2}\right.}}
$$




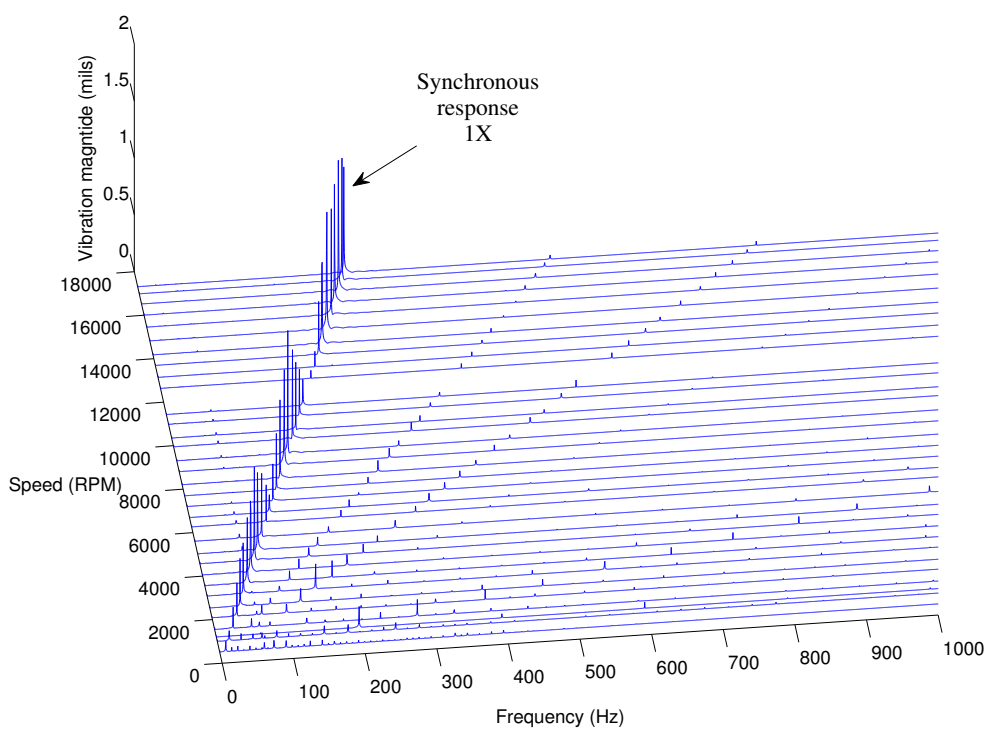

(a) $\mathrm{Q}=0 \mathrm{MN} / \mathrm{m}$

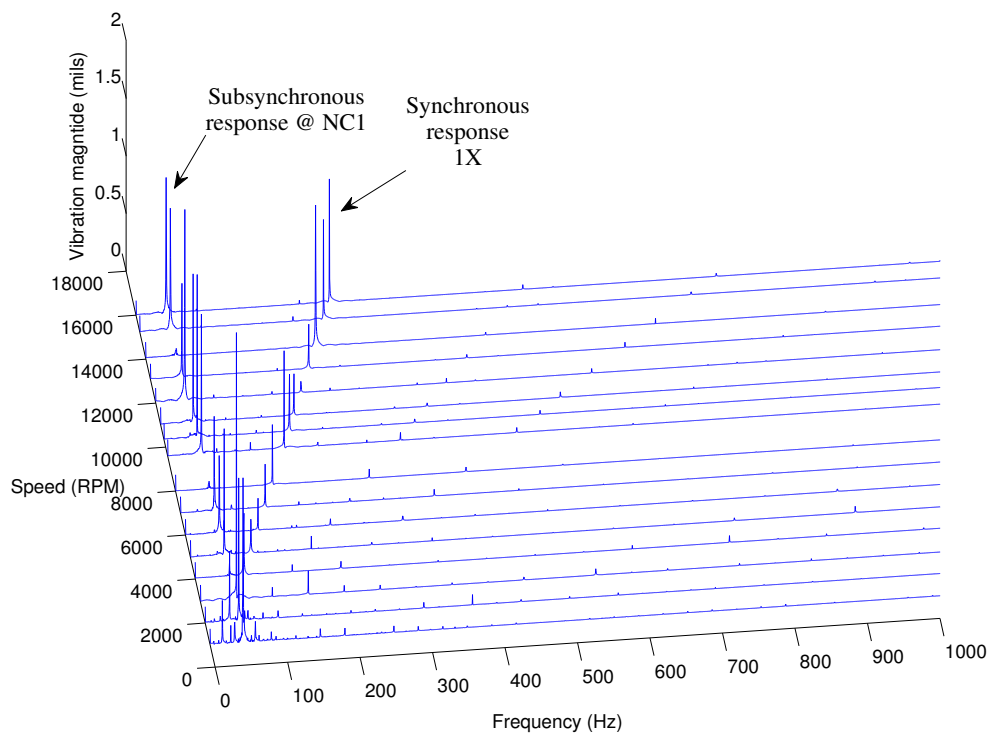

(b) $Q=1600 \mathrm{~N} / \mathrm{mm}$

Figure 3.4: Waterfall plots of vibration spectra from MBTRI rotor with and without midspan CCS showing peaks due to the subsynchronous vibrations at first rotor mode $\mathrm{Nc1}$ and the synchronous rotor response. 
and the minimum log dec specified by the API standards for centrifugal compressors in oil and gas service is 0.1 , corresponding to a damping ratio of 0.0159 (API 684, 2005; Vance et al., 2010). This margin of safety is necessary to account for uncertainties in the modeling and stability analysis.

For typical rotor-bearing systems the mode corresponding to the lowest system natural frequency (Nc1) is affected by the destabilizing mechanism (Lund, 1975). The goal is to accurately identify $\zeta_{i}$ for $\mathrm{Nc1}$ while the rotor is spinning, i.e., in the presence of damped and undamped sinusoids and random noise. By measuring the free decay of the rotor vibration following the removal of excitation, e.g., an impulse, the damping ratio may be obtained using output only time-domain measurements. Estimation techniques based on backward auto-regression (Kumaresan \& Tufts, 1982) using single sensor measurements are known as single degree of freedom (SDOF) and are commonly used for experimental rotor stability testing (Tasker \& Chopra, 1990). The modal selectivity and signal-to-noise ratio can be further improved by using blocking tests, which can isolate $\mathrm{Nc1}$ by providing forward circular excitation at its precise natural frequency until a suitable amplitude response is obtained. Following the termination of blocking, the free decay of the vibrations can be captured by the position sensors and fed into the offline auto-regression algorithm to estimate $\zeta_{1}$ with minimal variance. In cases where the damping ratio is higher, SNR is low, or multiple closed spaced modes are present SDOF estimation techniques have reduced accuracy. Cloud et al. (2009) describe a multiple degree of freedom technique (utilizing more than one sensor channel) based on vector backward auto-regression of time domain measurements to estimate $\zeta_{i}$ of multiple modes following termination of excitation. Figure 3.5 shows the free decay of vibrations following the termination of forward circular blocking excitation at $42 \mathrm{~Hz}$, while the rotor is spinning at 7,000 rpm. The dominant damped frequency during free decay was $44.7 \mathrm{~Hz}$ and damping ratio and log decrement were estimated to be $2.9 \%$ and 0.18 , respectively. 


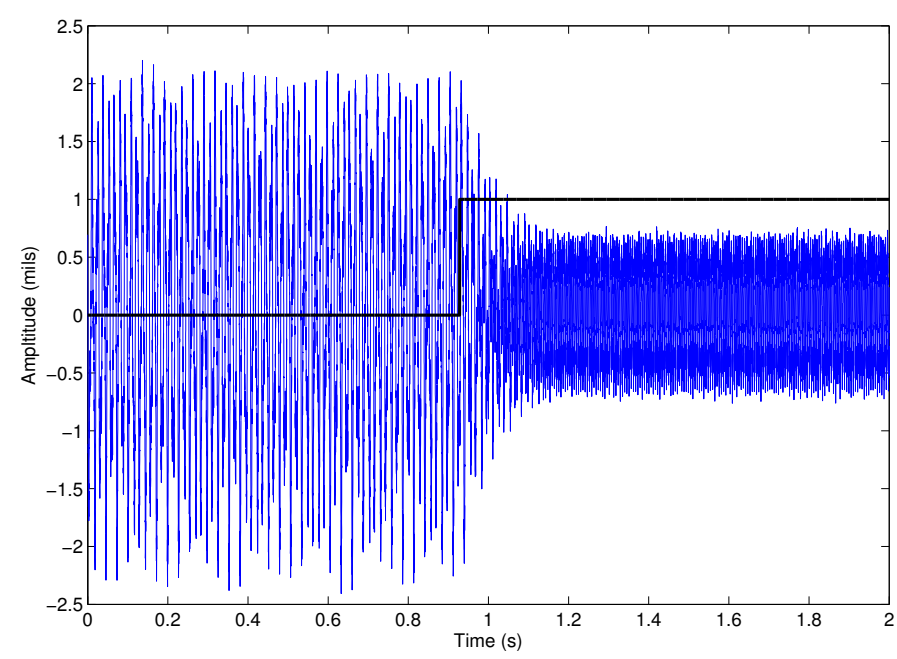

Figure 3.5: The free decay of rotor vibrations following termination (indicated by the rising edge trigger signal) of forward circular blocking excitation while rotor spins at 7,000 rpm.

\subsubsection{Factors Affecting the Onset of Instability}

Studies on the stability of turbomachinery with fluid film journal bearings found that an optimum level of effective damping $\hat{c}$ at a given operating point, i.e., rotating speed, torque, discharge pressure, must exist given that each operating point has a maximum allowable value of destabilizing aerodynamic excitation before OSI (Barrett, 1979). By parametrically varying the damping $c$ provided by the bearings, and performing a stability analysis, a solution $\hat{c}$ which maximizes the log decrement of $\mathrm{Nc} 1$ can be found (Vance et al., 2010). An important factor governing rotordynamic performance is the bearing-shaft stiffness ratio $K_{\mathrm{r}}$, which is the ratio of the total support stiffness in a single plane $k_{\mathrm{b}}$ to the shaft stiffness $k_{\mathrm{s}}$

$$
K_{\mathrm{r}}=\frac{2 k_{\mathrm{b}}}{k_{\mathrm{s}}}
$$

In the case of mechanical bearings, $k_{\mathrm{b}}$ is a function of geometry and lubricant properties, while for magnetic bearings, $k_{\mathrm{b}}$ is a largely a function of the control law. The choice of $k_{\mathrm{b}}$ depends on the magnitude of the operating loads of the rotor, while the choice of $k_{\mathrm{S}}$, which is a function of the rotor material and dimensions, is governed by considerations for the driving torque applied by the motor or engine. Elastic beam theory predicts a lateral 
stiffness for a rotor of uniform cross section

$$
k_{\mathrm{s}}=\frac{48 E I}{L^{3}},
$$

where $E$ is Young's modulus, $I$ is the area moment of inertia and $L$ is the shaft length (Inman, 2006). A flexible rotor with respect to its bearings may be characterized with $K_{\mathrm{r}}$ greater unity, indicating a fair degree of shaft deflection due to the greater bearing stiffness. Such motion may be highly desired as it reflects the possibility of larger effective damping of vibrations (as $c$ is proportional to velocity). Assuming symmetric support properties, the optimum damping can be estimated knowing the modal mass of the rotor $M_{\mathrm{m}}$, rigid bearing critical speed $\omega_{c r}$, and $K_{\mathrm{r}}$ as

$$
\hat{c}=M_{\mathrm{m}} \omega_{\mathrm{cr}}\left(\frac{1+K_{\mathrm{r}}}{2}\right) .
$$

Assuming the presence of optimum damping in the rotor-bearing system, Barrett derived the maximum allowable aerodynamic excitation $Q_{\max }$ as a function of $M_{\mathrm{m}}$ and $\omega_{\mathrm{cr}}$ and $K_{\mathrm{r}}$

$$
Q_{\max }=\frac{M \omega_{\mathrm{cr}}^{2}}{2\left(1+K_{\mathrm{r}}\right)} .
$$

Therefore, $Q_{\max }$ may be raised by either increasing the rotor mass, increasing $\omega_{\mathrm{cr}}$, or reducing $K_{\mathrm{r}}$. Current trends in the design of high-pressure compressors are producing lighter weight shafts with higher $K_{\mathrm{r}}$ leading to lower stability threshold and machines which are more susceptible to aerodynamic excitation. If suboptimal damping $c<\hat{c}$ is present in the rotor-bearing system then the onset of instability begins at CCS values less than $Q_{\max }$.

The effect of bearing stiffness anisotropy on the stability threshold has been studied in fluid-film bearings by several workers (Childs, 1993; Ehrich, 2004). The accepted observation is that increasing the difference between the vertical and horizontal principle direct support stiffness $\left(k_{x x}\right.$ and $k_{y y}$ ) leads to asymmetric whirl orbits. Compared to circular whirl orbits, asymmetric orbits are less effective at destabilizing Nc1. The result is a higher insta- 
bility threshold. However, the drawback is a larger synchronous response amplitude along the axis with reduced stiffness (Vance et al., 2010). Black (1976) also pointed out that for smaller machines, often with tighter margins for bearing load capacity, the pursuit of optimum damping should precede the introduction of bearing stiffness anisotropy.

A major advantage of AMBs over passive mechanical bearings is their ability to produce customized stiffness and damping characteristic and exert direct control over several aspects of the dynamics of the rotor-bearing system (Habermann \& Brunet, 1984). Furthermore, the damping and stiffness properties may be modified on-the-fly according to an available (either measured or estimated) scheduling parameter, e.g., operating speed, or as needed over the lifetime of the machine. The ideas of optimum damping and support anisotropy can be integrated into the existing design methodology for optimizing the performance of the rotor-bearing system.

\subsection{A Control Systems Perspective}

\subsubsection{Rotor-AMB System Description}

The rotor-AMB system without a feedback controller is an open-loop unstable plant as a result of the negative stiffness of the electromagnetic actuator. The real poles representing the rigid body modes of the rotor are shifted from the origin to the right half-plane (RHP) and the frequency of these unstable poles is proportional to the AMB negative stiffness. The lightly damped flexible rotor modes are represented by alternating pairs of complex poles and zeros close to the imaginary axis. Assuming a general proper SISO transfer function of the form

$$
G(s)=\frac{N(s)}{D(s)}=k \frac{\prod_{i=1}^{m}\left(s-\beta_{i}\right)}{\prod_{j=1}^{n}\left(s-\alpha_{j}\right)},
$$


where $n \geq m$, the poles are the real or complex roots $\alpha_{j}$ of the denominator polynomial $D(s)$, and the zeros are the real or complex roots $\beta_{i}$ of the numerator polynomial $N(s){ }^{1}$. The poles and zeros of the open-loop plant play an important role in the achievable stability and transient response of the closed-loop system regardless of the control algorithm used (Goodwin et al., 2000). The bending modes are stable and Figure 3.6a shows the poles and zeros of a typical MIMO rotor-AMB system with a flexible rotor. Higher frequency poles and zeros due to the power amplifiers and additional filters may be ignored without loss of generality. The addition of destabilizing CCS to the rotor system has the following effects on the plant model as observed in Figure 3.6b:

1. The real unstable pole corresponding to the first rigid body mode ( $\mathrm{Nc} 1)$ splits into a pair of complex conjugate poles and moves rightwards with increasing CCS magnitude.

2. The complex pole-zero pairs belonging to the first bending mode ( $\mathrm{Nc} 3$ ) each split into poles and zeros that move to the left and right of their original location.

At zero rotation speed and prior to the introduction of the CCS, the lateral motion of the rotor in the $x$ and $y$ directions can be considered largely decoupled. The pole-zero splitting described above arises from the interaction introduced between the lateral $x$ and $y$ motion of the rotor. A similar phenomenon occurs with the speed dependent gyroscopic coupling of the rotor at high rotation speeds. Whilst the gyroscopics usually have some affect on all the rotor modes depending on the construction of the rotor, the CCS overwhelmingly affects the first rigid body and first bending modes.

As Figure 3.7a shows, the addition of stabilizing feedback has a profound effect on the open-loop poles. In Figure 3.7a the control law stabilizes the unstable rigid body modes

\footnotetext{
${ }^{1}$ The definition holds true only if $N(s)$ and $D(s)$ are coprime. For an extension to MIMO systems, we introduce the rational transfer function matrix $G(s)$. If $\lambda_{p}$ is a pole of any entry within $G(s)$, then it must be a pole of $G(s)$. Multivariable zeros are grouped into transmission zeros or blocking zeros. $\lambda_{z b}$ is a blocking zero of $G(s)$ if it is a zero of every nonzero element of $G(s)$, i.e. $G\left(\lambda_{b z}\right)=0 . \lambda_{t z}$ is a transmission zero if it is a zero of at least one element of $G(s)$, i.e. $G\left(\lambda_{t z}\right) \neq 0$ (Chen, 1998).
} 
and supplements the damping of the flexible modes. As a result, all the poles are moved into the left half-plane (LHP). In some special cases, the controller may use RHP zeros to shift the phase in the vicinity of bending modes for damping purposes ( $\mathrm{Li}, 2006)$. As expected, the zeros of the plant are not affected by feedback and remain in the LHP.

The effect of increasing magnitudes of destabilizing CCS acting on the closed-loop plant is shown in Figure 3.7b. From the pole-zero map it is evident that for low levels of CCS the closed-loop system remains stable with all poles in the LHP and only the controller zeros in the RHP. Increasing levels of CCS have the effect of:

1. Causing the complex pole pair belonging to $\mathrm{Nc} 1$ to separate into one locus moving further into the LHP (increased stability of the backward mode) and another locus moving into the RHP (decreased stability of the forward mode).

2. Causing the complex zero pair belonging to $\mathrm{Nc} 3$ to separate into one locus moving further into the LHP and another locus moving into the RHP. Note that Nc3 zero crosses into the RHP before the Nc1 pole.

3. To a much lesser extent, poles and zeros belonging to other rotor modes also split. However, none of the loci enter the RHP.

This picture agrees with the mechanical perspective of a rotor-bearing system approaching instability namely, the frequency of the forward mode of $\mathrm{Nc1}$ increases and its damping ratio falls, while the frequency of the backward mode of $\mathrm{Nc} 1$ falls and its damping ratio rises all with increasing destabilizing force. The onset of instability is marked by minimum level of CCS required for departure of the Nc1 pole from the LHP. Changing the feedback control law may alter the $\mathrm{Nc1}$ pole zero trajectory and the stability threshold by delaying OSI. However, feedback is limited by its inability to alter the trajectory of the Nc3 zeros. 


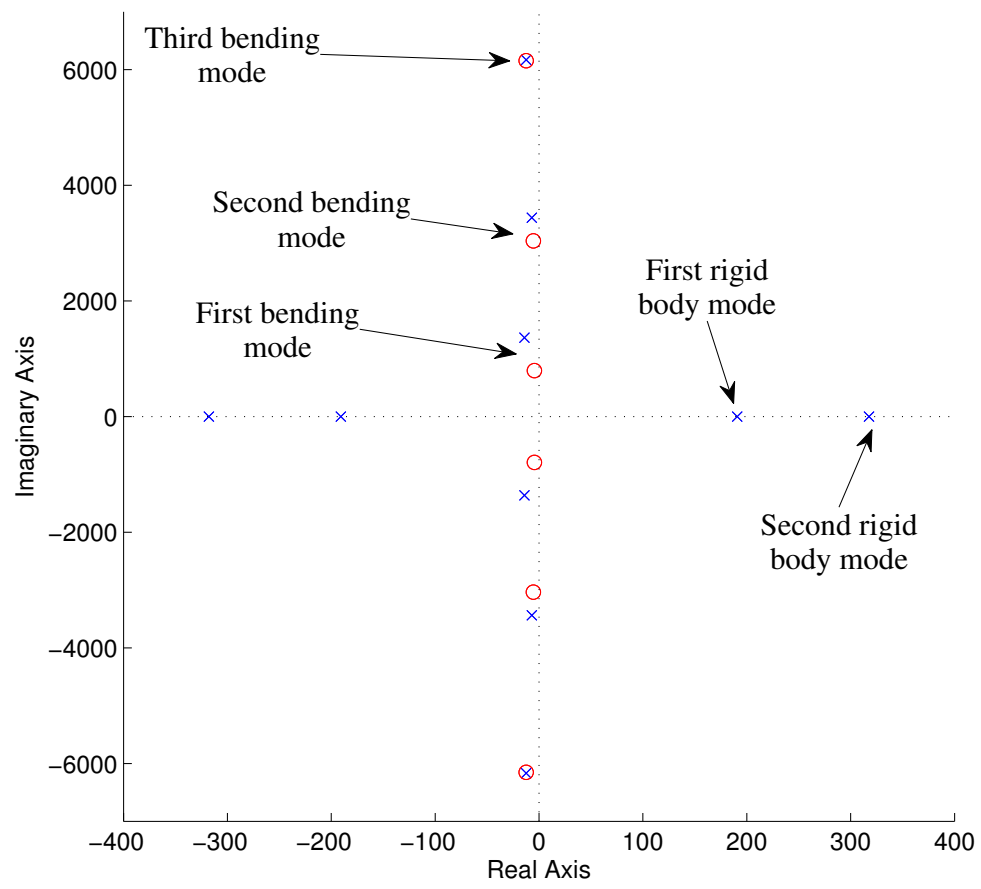

(a) Without any CCS.

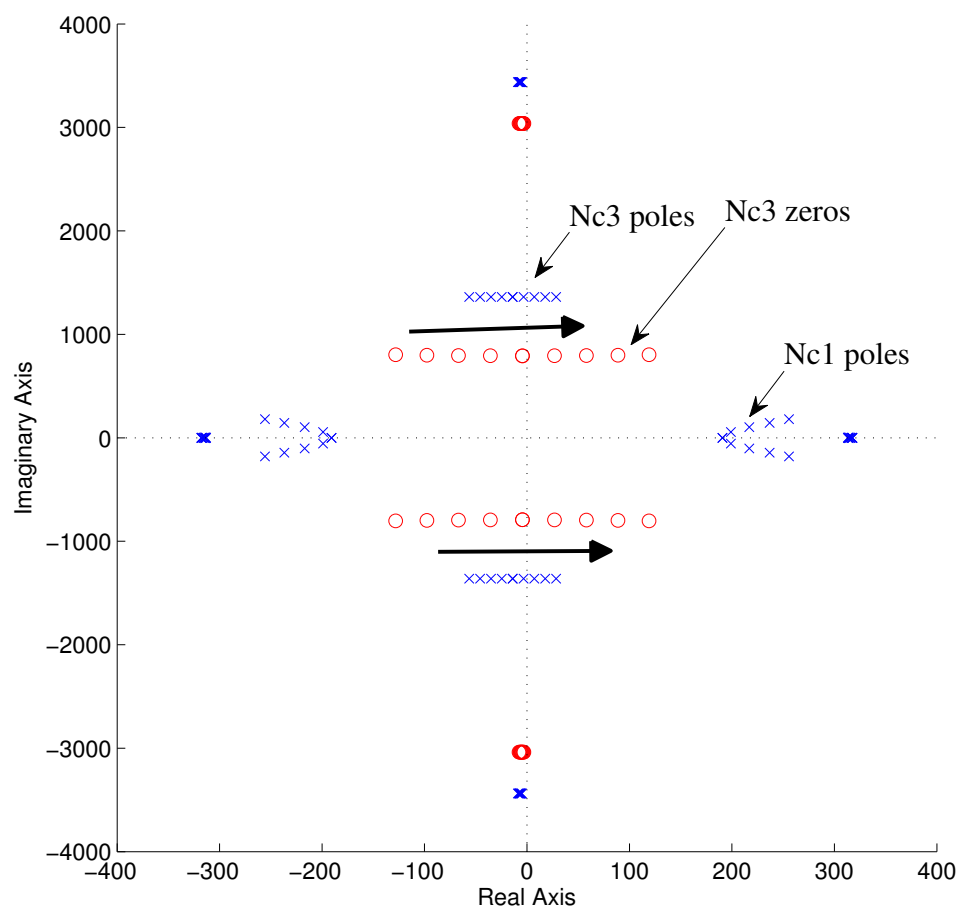

(b) Pole-zero trajectory with destabilizing CCS (0 to $2400 \mathrm{~N} / \mathrm{mm}$ ).

Figure 3.6: Pole-zero map of open-loop rotor-AMB system at $0 \mathrm{rpm}$ with and without CCS. 


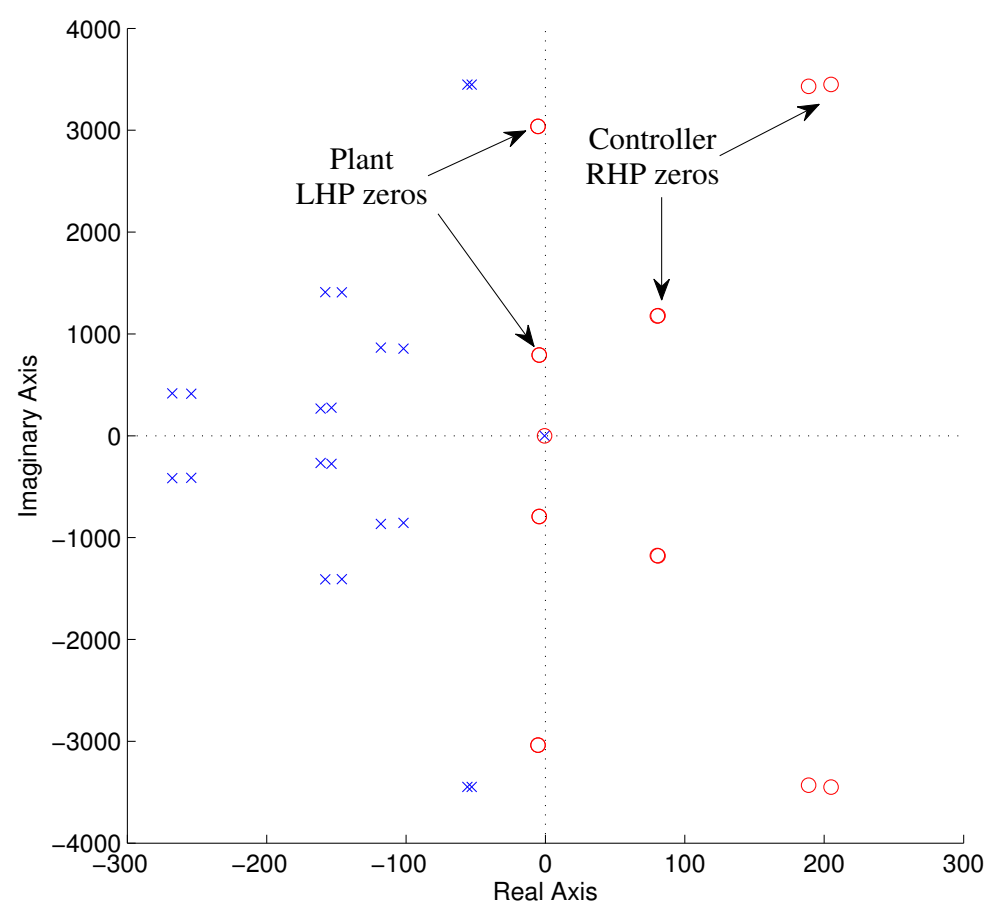

(a) Without CCS.

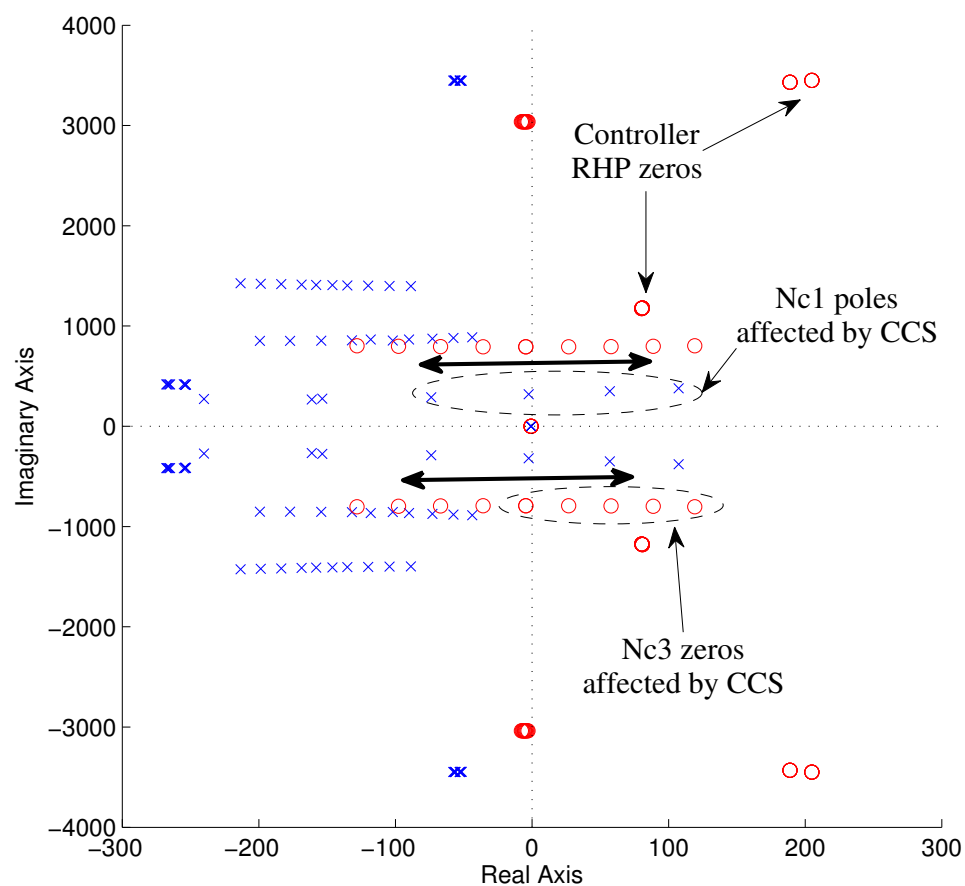

(b) Pole-zero trajectory with destabilizing CCS (0 to $2400 \mathrm{~N} / \mathrm{mm}$ ).

Figure 3.7: Pole-zero map of closed loop rotor-AMB system at $0 \mathrm{rpm}$ with and without CCS. 


\subsubsection{Fundamental Control Limitations}

The pole and zero loci in the open-loop and closed-loop models of the rotor-AMB system provide critical information for the analysis of fundamental control limitations facing the system. Bandwidth limitations arising from the presence of poles and zeros in the RHP are discussed in this section from a classical viewpoint. The frequency domain provides a powerful tool to study these limitations in the form of numerous closed-loop system sensitivity functions. Results pertaining to SISO systems are introduced for clarity before a generalization to MIMO systems.

Some examples of systems with RHP poles by design include several modern military fighter aircraft with the property of relaxed aerodynamic stability such as the X-29 prototype, F-16, F-117, F-22 and Eurofighter Typhoon. Relaxes stability entails that under certain flight conditions the aircraft's center of gravity is behind the center of aerodynamic pressure and in the absence of a suitable control input will oscillate with increasing amplitude (Abzug \& Larrabee, 2002). A digital flight control system is mandatory for such aircraft as the human pilot simply cannot respond fast enough to provide this stabilizing control input. RHP zeros are frequently encountered in boost DC-DC converters where their presence reduces the maximum gain bandwidth of the converter (Mitchell, 2001).

\subsubsection{Unstable plants (Limitations due to RHP poles)}

The fundamental conservation law in control system analysis and design takes the form of the Bode sensitivity integral (Bode, 1945). Through feedback, the reduction of sensitivity function ${ }^{2}$ magnitude $(|S(j \omega)|<1)$ in a given frequency band is accompanied by enhanced disturbance attenuation in that band and an increase in sensitivity or reduction of performance $(|S(j \omega)|>1)$ in another frequency band (see Figure 3.8). It is not possible to achieve arbitrary disturbance rejection across at all frequencies, therefore a trade-off is necessary. Furthermore, the extent of achievable disturbance attenuation and closed-loop performance

\footnotetext{
${ }^{2}$ For a SISO system $G(s)$ stabilized by feedback control $K(s)$, the sensitivity function $S(s)=1 /(1+G(s) K(s))$.
} 


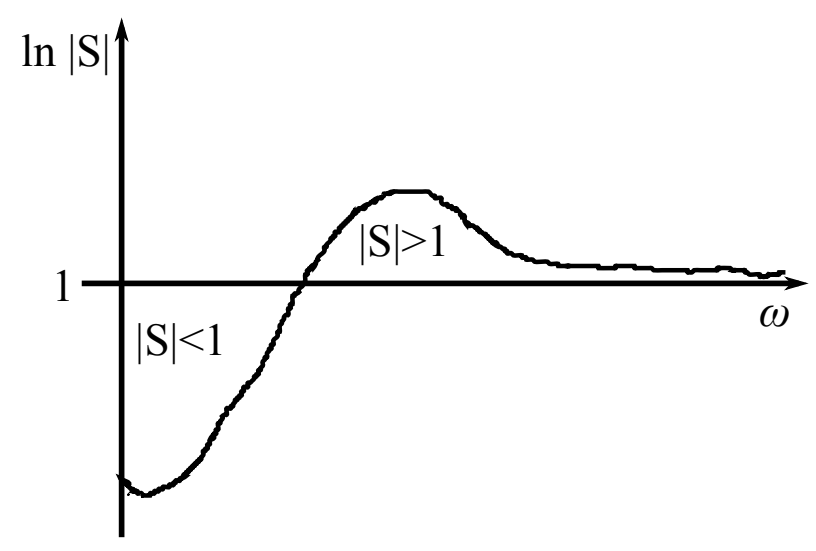

Figure 3.8: Magnitude plot of a typical sensitivity function illustrating the trade-off in disturbance rejection due to the conservation of the sensitivity integral.

improvement depends intimately on the properties of the plant. For open-loop stable plants the conservation law states that the infinite integral of the log magnitude of the sensitivity function is zero

$$
\int_{0}^{\infty} \ln |S(j \omega)| d \omega=0,
$$

This equation reveals that the net area subtended by the logarithm of the sensitivity function and the horizontal axis is zero for stable plants. Therefore, the area of sensitivity reduction (i.e. improved disturbance rejection, is exactly balanced by the area of sensitivity increase, i.e., deterioration in performance). Freudenberg \& Looze (1985) extended this result to cover open-loop unstable plants successfully stabilized by feedback control. The net area of the integral of log sensitivity is equal to a positive constant

$$
\int_{0}^{\infty} \ln |S(j \omega)| d \omega=\pi \sum_{i=1}^{n_{p}} \operatorname{Re}\left(p_{i}\right)
$$

where $n_{p}$ is the number of RHP poles in the plant and controller. In this case, the area of sensitivity increase exceeds the area of sensitivity reduction resulting in a net positive value. Therefore, the achievable sensitivity reduction in unstable systems is limited by 
the the multiplicity and magnitude of the RHP poles (Sidi, 2001). In practical systems, the control bandwidth (read, frequency over which sensitivity can be affected) is finite and denoted as the available bandwidth $\Omega_{a}$. Notably, $\Omega_{a}$ is not related to the structure of control law or design methodology followed, but an a priori restriction due to the available hardware, including but not limited to sensor bandwidth, actuator small signal and power bandwidths, and unmodeled plant dynamics (Stein, 2003). The resulting restatement of Bode sensitivity integral for practical open-loop stable and open-loop unstable plants with finite control bandwidth is

$$
\int_{0}^{\Omega_{a}} \ln |S(j \omega)| d \omega=\delta
$$

and

$$
\int_{0}^{\Omega_{a}} \ln |S(j \omega)| d \omega=\pi \sum_{i=1}^{n_{p}} \operatorname{Re}\left(p_{i}\right)+\delta,
$$

where $\delta$ is the remaining sensitivity which cannot be reduced using feedback as $\omega>\Omega_{a}$. As mentioned before, the location of the RHP poles in a rotor-AMB system depends on the rotor mass and the negative stiffness of the AMB actuator. As was shown in Figure 3.6b, the presence of CCS further displaces the unstable poles into the RHP with the effect of:

1. increasing the minimum achievable peak sensitivity $s_{\min }$,

2. increasing the minimum control bandwidth for stability, $\omega_{c}$.

The effect of $\Omega_{\mathrm{a}}$ on the $s_{\min }$ for an unstable plant with a single real pole at $p_{1} \mathrm{rad} / \mathrm{s}$ can be demonstrated using a simple sensitivity function prototype in the form of a trapezoid shown in Figure 3.9. The sensitivity function at low frequencies has a slope of +1 and peaks at a frequency of $\Omega_{m} \mathrm{rad} / \mathrm{s}$ and a minimum peak sensitivity of $s_{\min }$. This minimum can be 


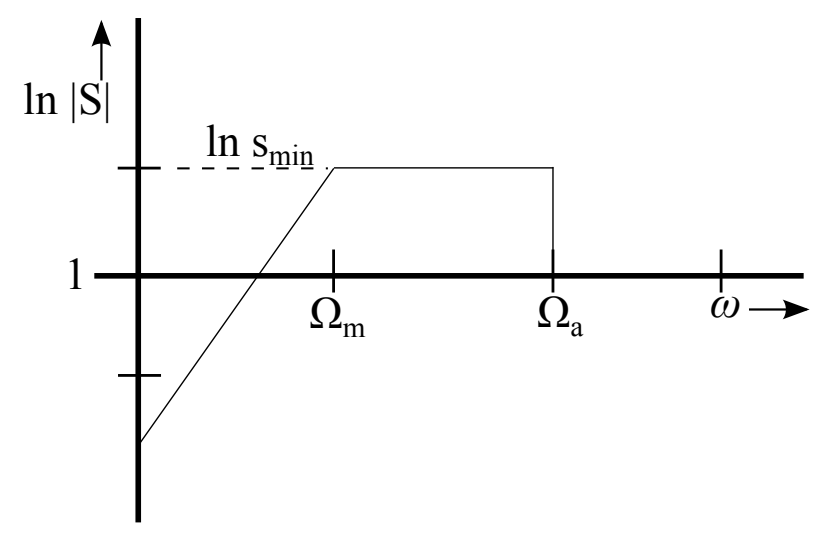

Figure 3.9: A sensitivity function template for a plant with an unstable pole at $p_{1} \mathrm{rad} / \mathrm{s}$ and achievable bandwidth of $\Omega_{\mathrm{a}} \mathrm{rad} / \mathrm{s}$.

derived by applying the Bode sensitivity integral

$$
\begin{aligned}
\int_{0}^{\Omega_{a}} \ln |S(j \omega)| d \omega & =\pi p_{1}+\delta, \\
\int_{0}^{\Omega_{\mathrm{m}}} \ln \left(\frac{\omega s_{\min }}{\Omega_{\mathrm{m}}}\right) d \omega+\left(\Omega_{\mathrm{a}}-\Omega_{\mathrm{m}}\right) \ln \left(s_{\min }\right) & =\pi p_{1}+\delta, \\
\Omega_{\mathrm{a}} \ln \left(s_{\mathrm{min}}\right)+\Omega_{\mathrm{m}} & =\pi p_{1}+\delta,
\end{aligned}
$$

and

$$
s_{\min }=\exp \left(\frac{\Omega_{\mathrm{m}}+\pi p_{1}+\delta}{\Omega_{\mathrm{a}}}\right) .
$$

If closed-loop robustness specifications are provided in the form of gain and phase margins (as is often the case in aircraft flight control systems, for example). $s_{\min }$ can provide expressions for the achievable gain and phase margins ${ }^{3}$. If the achievable bandwidth is known, these expressions provide quick tests to evaluate the feasibility of any control law meeting the specification (Stein, 2003). The ISO stability specification for AMB systems specifies minimum peak sensitivity values for machines (ISO 14839-3, 2006).

\footnotetext{
${ }^{3}$ Gain Margin $\geq \frac{s_{\min }}{s_{\min }-1}$, and Phase Margin $\geq 2 \sin ^{-1}\left(\frac{1}{2 s_{\min }}\right)$ (Sidi, 2001).
} 
A second profound limitation on the benefits of feedback due to presence of an RHP pole is revealed through the analysis of the loop transmission or open-loop gain transfer function using the Bode gain-phase theorem. The theorem states that for any stable, minimum-phase ${ }^{4}$ system $G(j \omega)$, the phase $\angle G(j \omega)$ is uniquely related to the magnitude $|G(j \omega)|$ (Bode, 1945). The loop gain for an arbitrary stable minimum-phase system is shown in Figure 3.10. The loop gain $(L(s)=G(s) K(s)$ where $K(s)$ and $G(s)$ are the controller and plant transfer functions, respectively) reveals the extent to which feedback can improve the stability and performance of a given system (Franklin et al., 1994). Note that the output sensitivity function $S(s)=1 /(1+L(s))$. Therefore, a large magnitude loop gain corresponds to a good sensitivity reduction. A significant simplification for rotor-AMB systems with flexible rotors since each flexible mode adds a peak to the loop gain which results in multiple $0 \mathrm{~dB}$ crossings, and multiple GMs and PMs. Nevertheless, $\omega_{\mathrm{co}}$ relates to the bandwidth of the controller, each PM determines the level of damping for each mode, and the low frequency gain of $L(j \omega)$ corresponds to the steady state disturbance rejection, i.e., stiffness of the closed loop system. The loop gain $L_{1}(s)$ of a typical rotor-AMB system with a single real unstable pole at $a \mathrm{rad} / \mathrm{s}$ can be decomposed into a stable minimum phase component $L_{\mathrm{MP}}(s)$ through the following steps

$$
L_{1(s)}=\frac{L_{2}(s)}{s-a}=\frac{L_{2}}{s+a} \frac{s+a}{s-a}=L_{\mathrm{MP}}(s) \frac{s+a}{s-a} .
$$

where it is apparent that $\left|L_{1}(j \omega)\right|=\left|L_{\mathrm{MP}}(j \omega)\right|$. However, from the Bode gain-phase relationship

$$
\angle\left(L_{1}(j \omega)\right)=\angle\left(L_{\mathrm{MP}}(j \omega)\right)-\pi+2 \arctan (\omega / a) .
$$

The presence of the RHP introduces phase lag of up to $180^{\circ}$ at low frequencies because $\arctan (x)$ tends to 0 with falling $x$. This large phase lag requires an increased control effort

\footnotetext{
${ }^{4}$ Minimum-phase (MP) implies that a system displays the least possible phase lag for a given magnitude response $|L(j \omega)|$. A non minimum-phase (NMP) system exhibits more phase lag than a minimum-phase system with an identical magnitude response $|L(j \omega)|$. The extra lags are due to the presence of RHP zeros or time delays (Skogestad \& Postlethwaite, 2005).
} 


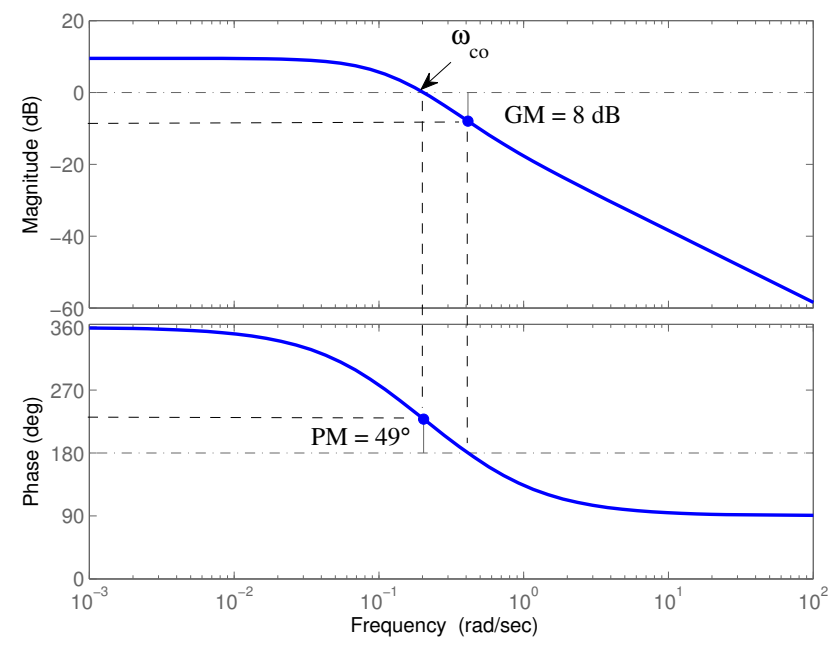

Figure 3.10: The loop gain of an arbitrary stable, minimum phase system showing the crossover frequency and the stability margins.

and specifically a larger crossover frequency $\omega_{\text {co }}$ before the system can be stabilized. On the other hand, at high frequencies the phase lag disappears as $\arctan (x)$ tends to $\pi / 2$ with rising $x$. With an increased $\omega_{\text {co }}$, a higher minimum control bandwidth can lead to problems with unmodeled dynamics and amplification of measurement noise. In conclusion, the effect of the RHP pole on the closed-loop system is to introduce minimum achievable control bandwidth restrictions and minimum peak sensitivity limitations.

\subsubsection{Non Minimum-Phase Plant (Limitations due to RHP Zeros)}

The effect of a complex RHP zero $(z=a \pm j b)$ on the sensitivity is particularly obvious when considered in a plant containing $n_{p}$ RHP poles $p_{i}$. For the resulting stable closedloop system the ideal weighted sensitivity integral is

$$
\int_{0}^{\infty} \ln |S(j \omega)| \cdot w(z, \omega) d \omega=\pi \cdot \prod_{i=1}^{n_{p}}\left|\frac{p_{i}+z}{\bar{p}_{i}-z}\right|
$$

where $w(z, \omega)=\frac{a}{a^{2}+(b-w)^{2}}+\frac{a}{a^{2}+(b+w)^{2}}$ (Skogestad \& Postlethwaite, 2005). The weighting function $w(z, \omega)$ takes into account the rising performance penalty introduced as the loca- 
tion of the zero coincides with the frequency range where high performance is desired. Two conclusions can be made from Equation (3.23). First the presence of a RHP zero increases the minimum achievable sensitivity. Second, increased proximity of the RHP zero to the RHP poles leads to the RHS becoming very large, reflecting the fact that the cancellation of a RHP pole by a RHP zero leads to an system that feedback cannot stabilize.

In the same manner as the plant with a RHP pole, the loop gain $L_{\mathrm{NMP}}(s)$ for a plant with a RHP zero at $\lambda_{1} \mathrm{rad} / \mathrm{s}$ may be decomposed into its minimum phase components $L_{\mathrm{MP}}(s)$

$$
L_{\mathrm{NMP}}(s)=L_{3}(s)\left(s-\lambda_{1}\right)=L_{3}(s)(s+b) \frac{s-\lambda_{1}}{s+\lambda_{1}}=L_{\mathrm{MP}}(s) \frac{s-\lambda_{1}}{s+\lambda_{1}}
$$

where it is apparent that $\left|L_{\mathrm{NMP}}(j \omega)\right|=\left|L_{\mathrm{MP}}(j \omega)\right|$ (Franklin et al., 1994). However, the Bode gain-phase relation reveals that

$$
\angle\left(L_{\mathrm{NMP}}(j \omega)\right)=\angle\left(L_{\mathrm{MP}}(j \omega)\right)-2 \arctan \left(\frac{\omega}{a}\right) .
$$

At high frequencies, the phase lag on the loop gain approaches $180^{\circ}$, while at low frequencies there is a minimal phase lag since $\arctan (x)$ tends to zero. It can be concluded that the presence of RHP zeros introduces an upper limit on the crossover frequency, beyond this limit the benefits of feedback are no longer enjoyed.

The location of system zeros is intimately tied to the quality of the observation of its internal states as reflected by the number and location of sensors. Therefore, by adding more sensors to the plant one may eliminate some RHP zeros. Implementing a state observer to recover state information, however, will not suffice (Astrom \& Wittenmark, 1997).

\subsubsection{Unique Limitations of AMBs}

A complete AMB actuator consists of one or more power amplifiers supplying current to stator windings (with a ferromagnetic core) which attract the rotor. The power amplifier and AMB rotor/stator properties must be matched to maximize performance of the overall 
actuator. The actuator has several unique force output limitations arising from electromagnetic effects as well as requirements for accurate sensor feedback of the rotor position (Schweitzer, 2002). These limiting effects are particularly important as their onset is accompanied by deterioration of the expected actuator stiffness and damping properties regardless of the type of control algorithm used (Maslen et al., 1988).

Considering a pair of magnetic actuators around a rotor as depicted in Figure 3.12, and assuming bias current linearizaton, the static force output as a function of current and position of the rotor is

$$
F_{x}=\alpha \frac{\mu_{0} A_{\mathrm{p}} N_{\mathrm{t}}}{4}\left(\frac{\left(I_{\mathrm{b}}+i_{\mathrm{p}}\right)}{(g-\alpha x)^{2}}-\frac{\left(I_{\mathrm{b}}-i_{\mathrm{p}}\right)}{(g+\alpha x)^{2}}\right) \approx K_{\mathrm{i}} i_{p}-K_{\mathrm{x}} x
$$

where $I_{\mathrm{b}}$ is the bias current chosen, $i_{\mathrm{p}}$ is the perturbation current, $N_{\mathrm{t}}$ is the number of windings in a quadrant, $A_{\mathrm{p}}$ is the quadrant pole face area, and $x$ is a small displacement about the equilibrium radial air gap $g$ (Schweitzer et al., 1994). The maximum static force available from the actuator is determined by $\min \left(I_{\max }, I_{\mathrm{sat}}\right)$ where $I_{\max }$ is the peak output current supported by the power amplifier and $I_{\text {sat }}$ is the current in the AMB which results in the saturation flux density $B_{\text {sat }}$ limit of the magnetic material. Typically, the power amplifier is selected to have $I_{\max } \leq I_{\text {sat }}$. The flux density produced within a ferromagnetic material subject to a magnetomotive force $(\mathrm{MMF})$, i.e., product of current and winding turns, follows a magnetization curve that is an intrinsic property of the magnetic material (Allaire et al., 1994). The magnetization curve, shown in Figure 3.11 for silicon-iron, has a linear region where flux density $B$ is approximately proportional to MMF. This is the desired operating region of the AMB. Outside this region, the curve tapers off so a successively larger MMF, i.e., more current, is required per unit increase in $B$. Assuming $I_{\max }<I_{\mathrm{sat}}$, the dimensions of the AMB stator and rotor are sized to deliver a maximum static force

$$
F_{\text {max }}=\alpha \frac{B_{\mathrm{sat}}^{2} A_{\mathrm{p}}}{\mu_{0} A_{\mathrm{g}}}
$$




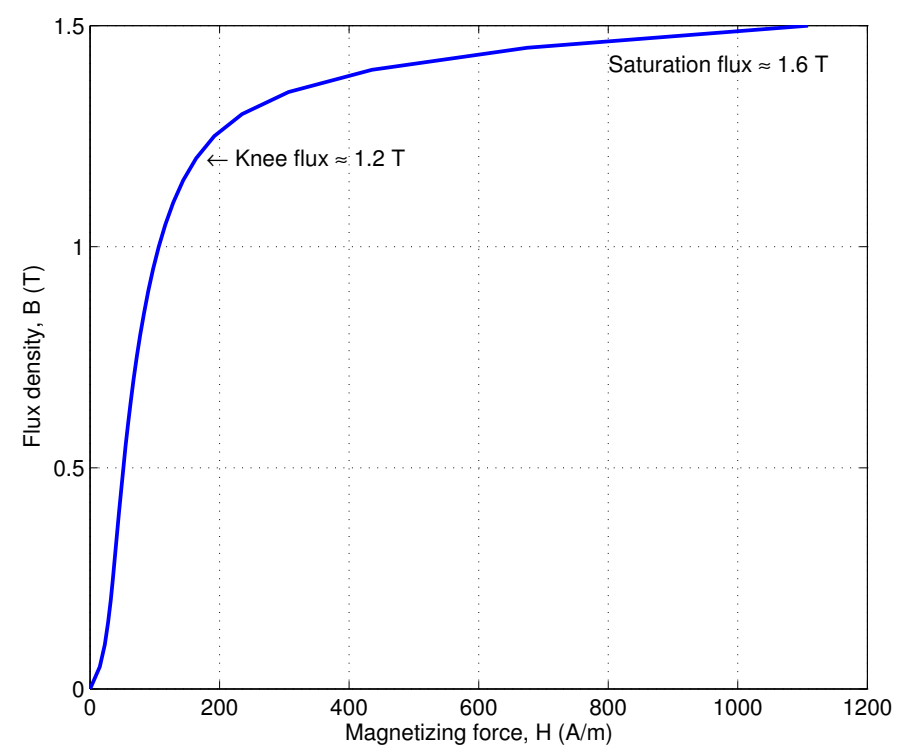

Figure 3.11: A magnetization curve for silicon-iron showing the knee flux density of approximately $1.2 \mathrm{~T}$ (Meeker, 2009).

where $A_{\mathrm{g}}$ is the area of the air gap normal to the rotor-stator flux path. If the suspended rotor is subject to a load exceeding $F_{\max }$, the AMB will not be able to maintain the rotorstator clearance and contact will occur between the rotor and backup mechanical bearing regardless of the action of the control algorithm. As a result, prudent sizing of the AMB with respect to worst-case operating loads is of paramount of importance (Bornstein, 1991; Swanson et al., 2008). A priori knowledge of transient loads is difficult for new applications, resulting in over design of the load capacity to provide a suitable margin.

While the above maximum force limit holds true for static force delivered by the AMB in response to constant current commands from the controller and location of the rotor at the center of the magnetic gap, practical operation requires the dynamic response of the actuator to be evaluated in terms of varying current signals and displacement of the rotor approaching maximum clearance. The power amplifier can be considered a current-limited voltage source driving a primarily inductive load (Antila, 1998). The effects of finite power and small-signal bandwidths of the amplifier-winding pair, and variation in the AMB spring rate with position are considered.

Switching amplifiers have the advantage of higher efficiency and reduced power loss 


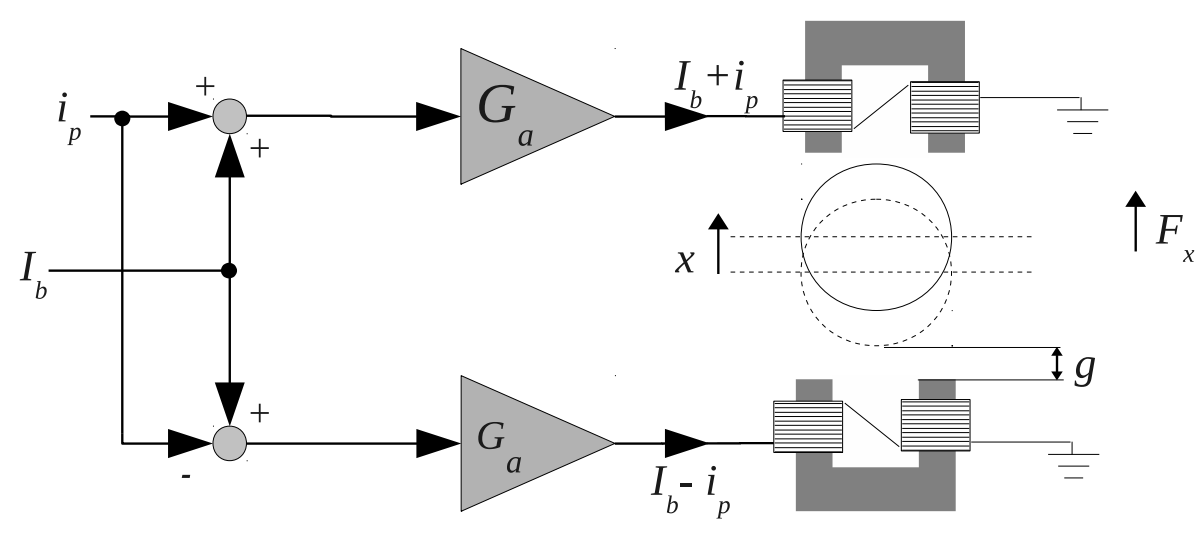

Figure 3.12: Differential amplifier-AMB driving mode producing bias current linearization of the AMB.

over their linear counterparts and are used extensively in AMB applications (Schroder, 1995). Without loss of generality, pulse-width modulation is used to command power switches to setup a potential difference $V_{\mathrm{w}}$ across the AMB windings in-order to drive the desired current $\left(I_{\mathrm{b}}+i_{\mathrm{p}}\right.$ or $\left.I_{\mathrm{b}}-i_{\mathrm{p}}\right)$ through the windings from a fixed supply voltage bus $V_{\mathrm{DC}}$. The impedance of the copper windings is dominated by a moderate to high inductance $L_{\mathrm{w}}$ while the winding resistance $R_{\mathrm{w}}$ is typically very low. The instantaneous voltage across the top windings in Figure 3.12 is

$$
V_{\mathrm{w}}=\left(I_{\mathrm{b}}+i_{\mathrm{p}}\right) R_{\mathrm{w}}+L_{\mathrm{w}} \frac{d i_{\mathrm{p}}}{d t}
$$

where $\frac{d i_{\mathrm{p}}}{d t}$ is the current slew rate. So long as $V_{\mathrm{w}}<V_{\mathrm{DC}}$, i.e., the power supply voltage exceeds the back EMF, the current slew rate limit of the amplifier is not exceeded and the actual output current tracks the current requested by the controller. In this scenario the AMB delivers the requested stiffness and damping effects and exhibits a force slew rate derived from Equation (3.26)

$$
\left.\frac{d F_{x}}{d t}\right|_{x=0}=K_{\mathrm{i}} \frac{d i_{\mathrm{p}}}{d t} .
$$

As the controller demands the current slew faster to respond to higher frequency vibrations 
eventually $V_{\mathrm{w}}>V_{\mathrm{DC}}$ and the amplifier can no longer overcome the back-EMF to allow the actual current to track the requested current. At this frequency, the current slew rate limit and corresponding force slew rate limits have been reached

$$
\begin{aligned}
\left.\frac{d i_{\mathrm{p}}}{d t}\right|_{\max } & \approx \frac{V_{\mathrm{DC}}-\left(I_{\mathrm{b}}+i_{\mathrm{p}}\right) R_{w}}{L_{\mathrm{w}}} \\
\left.\frac{d F_{x}}{d t}\right|_{\max } & \approx K_{\mathrm{i}}\left(\frac{V_{\mathrm{DC}}-\left(I_{\mathrm{b}}+i_{\mathrm{p}}\right) R_{w}}{L_{\mathrm{w}}}\right) .
\end{aligned}
$$

The resulting distortion of the current output signal introduces phase lag into the control loop which will result in the loss of stability. For a given maximum amplifier voltage, the maximum undistorted current delivered to the load can be plotted as a function of frequency. The area below the curve indicates operating points within the current slew rate limit. From this plot the power bandwidth $\omega_{\mathrm{pbw}}$, or the maximum frequency at which the amplifier can output its peak dynamic current can be found. $\omega_{\mathrm{pbw}}$ is not to be confused with the small signal bandwidth of the amplifier which is related to the current feedback loop within the amplifier and is typically several multiples higher than the power bandwidth. As the bias current is typically set to half the the maximum current, leaving the remainder for dynamic stiffness, the intersection of $0.5 I_{\max }$ with the curve marks the $\omega_{\mathrm{pbw}}$ as shown in Figure 3.13. The power bandwidth may only be expanded by reducing $L_{\mathrm{w}}$ or increasing the maximum amplifier power $\left(V_{\mathrm{DC}}\right.$ or $\left.I_{\max }\right)$. The power bandwidth defines the dynamic force capacity of the AMB and it should at least match the worst case dynamic loads, e.g., rotor imbalance at maximum speed of the machine. Note that other bandwidth limiting effects such eddy current losses in the rotor have been ignored (Zhu \& Knospe, 2010). In the case of radial AMBs, the laminated construction of the rotor and stator components greatly reduces losses due to electrodynamic effects. Hence, the treatment of eddy current losses may be safely ignored.

Displacement sensitivity is characterized by the reduction of bearing effective spring rate during large amplitude vibrations approaching bearing clearance (Maslen et al., 1988). 


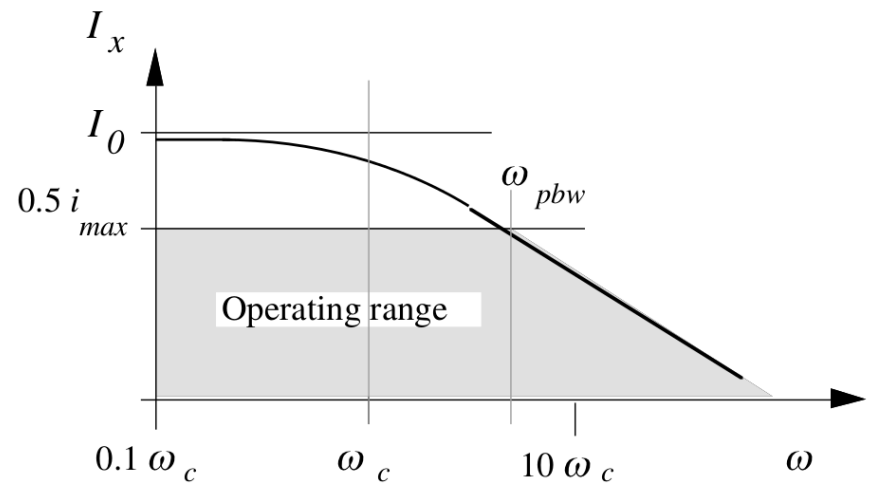

Figure 3.13: Magnitude plot illustrating the power bandwidth of an AMB-amplifier pair (Schweitzer, 2002).

This limitation provides impetus for improved control algorithms to better constrain the rotor to a tight orbit about its equilibrium position to avoid this nonlinear behavior.

\subsubsection{Stability Analysis}

Linear lateral stability analysis is important to expose problems which may lead to rotordynamic instability during design phases of new turbomachines. The current edition of the API 617 standard includes criteria for the stability analysis with respect to CCS arising from fluid-structure interactions at the bearings, impellers and seals in centrifugal compressors (API 617, 2002). The stability analysis of rotor-AMB systems differs from the typical analysis performed for rotors supported by passive mechanical bearings largely because the MIMO feedback controller has additional degrees of freedom and can produce a response that is a function of rotor displacement and velocity at multiple sensed locations. For instance, the response of mechanical bearing systems is strictly local, i.e., a function of the actuator rotor displacement and velocity at the bearing. Furthermore, passive mechanical rotor-bearing systems require stability to be confirmed only for modes within the operating speed range of the machine as the shaft rotation speed determines the response of the bearings. Thus supersynchronous modes are often ignored with passive mechanical supports. In strong contrast, the feedback controller in rotor-AMB systems has the ability 
to respond to any vibration detected by the sensors, and can potentially excite any mode within the actuator bandwidth (Williams et al., 1990). The actuator small-signal bandwidth is on the order of $1-3 \mathrm{kHz}$ and well above typical rotating speeds for industrial machinery. Therefore, supersynchronous modes have to be considered in the stability analysis so long as potential exists for the controller to destabilize them. Also, whereas the effects of passive mechanical bearings can be represented as frequency dependent stiffness and damping coefficients, generating similar coefficients for AMB system is almost guaranteed to produce incorrect results for stability analysis (Swanson et al., 2008). Factors such as sensor-actuator noncolocation, signal conditioning filters with steep transitions, sampling and computational delay, and control outputs produced by a multiplicity of measurement inputs cannot be represented by equivalent stiffness and damping coefficients.

To satisfy the above concerns, a simple model for the lateral stability analysis of a flexible rotor supported on AMBs based on a state-space description of the plant and a feedback controller is presented (Maslen \& Bielk, 1992). The results of the stability analysis are the damped natural frequencies (eigenvalues) and associated mode shapes (eigenvectors) of the rotor (Lund, 1974). The rotor state-space model developed from finite element analysis consists of mass $M$, stiffness $K$, damping $C$ matrices respectively. The speed dependent gyroscopic terms are included within $C$. The bearing force $F_{\text {amb }}$ acts on the rotor at the bearing locations $B_{\mathrm{amb}}$. The first-order state equation representing the Newtonian dynamics is

$$
\left[\begin{array}{cc}
I & 0 \\
0 & M
\end{array}\right]\left[\begin{array}{c}
\dot{q} \\
\ddot{q}
\end{array}\right]=\left[\begin{array}{cc}
0 & I \\
-K & -C
\end{array}\right]\left[\begin{array}{c}
q \\
\dot{q}
\end{array}\right]+\left[\begin{array}{c}
0 \\
B_{\mathrm{amb}}
\end{array}\right] F_{\mathrm{amb}}
$$

where the state vector $q$ represents lateral displacement of the rotor in two orthogonal planes. A destabilizing effect such as CCS may be introduced by adding appropriate entries to the matrices $K$ and $C$. The full coupled MIMO response of the feedback control law together with the dynamics of the transconductance power amplifier, displacement sensors, signal conditioning filters, and associated sampling and computational delay can be 
represented without loss of generality in the form

$$
\begin{aligned}
\dot{h} & =A_{\mathrm{h}} h+B_{\mathrm{h}} B_{\mathrm{s}} q \\
i & =C_{\mathrm{h}} h+D_{\mathrm{h}} B_{\mathrm{s}} q
\end{aligned}
$$

where $h$ is the state space for the control law, the matrix $B_{\mathrm{S}}$ represents the location of the displacement sensors in the state space $q$, and $i$ is the vector of output currents from the power amplifiers. Sensor-actuator noncolocation may be introduced if $B_{\mathrm{s}} \neq B_{\mathrm{amb}}$. The linearized force from the bearings is then

$$
\begin{aligned}
F_{\mathrm{amb}} & =-K_{\mathrm{i}} i-K_{\mathrm{x}} B_{\mathrm{amb}} q \\
& =-K_{\mathrm{i}} C_{\mathrm{h}} h-\left(K_{\mathrm{i}}+D_{\mathrm{h}} B_{\mathrm{s}}+K_{\mathrm{x}} B_{\mathrm{amb}}\right) q
\end{aligned}
$$

where the magnetic bearing parameters are $K_{\mathrm{i}}$ and $K_{\mathrm{x}}$. The complete system can be represented

$$
\left[\begin{array}{ccc}
I & 0 & 0 \\
0 & M & 0 \\
0 & 0 & I
\end{array}\right]\left[\begin{array}{c}
\dot{q} \\
\ddot{q} \\
\dot{h}
\end{array}\right]=\left[\begin{array}{ccc}
0 & I & 0 \\
-K-B_{\mathrm{amb}}\left(K_{\mathrm{x}} B_{\mathrm{amb}}-K_{\mathrm{i}} D_{\mathrm{h}} B_{\mathrm{s}}\right) & -C & -B_{\mathrm{amb}} K_{\mathrm{i}} C_{\mathrm{h}} \\
B_{\mathrm{h}} B_{\mathrm{s}} & 0 & A_{h}
\end{array}\right]\left[\begin{array}{c}
q \\
\dot{q} \\
h
\end{array}\right]
$$

The problem can be represented in the standard form of the generalized eigenvalue problem

$$
T^{-1} V \psi=\lambda \psi
$$

where $T$ is the LHS matrix from Equation (3.35) and $V$ is the RHS matrix. The solution found using standard eigensolvers allows the appropriate complex eigenvalues $\lambda$ and associated complex eigenvectors $\psi$ pertaining to the rotor physical displacement $q$ to be extracted. The eigenvalues reveal the damping ratio and natural frequency of modes in the physical coordinate space, while the eigenvectors reveal the rotor mode shape magnitude 
and phase information. This information can be used to generate two-dimensional and three-dimensional plots of the mode differentiating between forward and backward modes whirl modes as shown in Figure 3.14.

\subsection{Summary}

The preceding sections reveal the rich dynamics of a rotor-AMB experiencing rotordynamic instability. Optimum damping permits the estimation of the maximum destabilizing force and its parametrization in terms of constants such as the modal rotor mass, rigid bearing critical speed and the ratio of bearing stiffness to shaft stiffness. The predicted optimum damping can serve as a guide during the analysis of robust controllers, informing us whether room for improvement of a particular control algorithm exists. The effect of support anisotropy will also be investigated during the synthesis of robust controllers.

The classical results on the effects of RHP poles and RHP zeros on the achievable closed-loop performance take the form of limitations of lower and upper bounds on crossover frequency. The achievable bandwidth is another system property independent of the control law, which defines the minimum attainable peak sensitivity.The effects of RHP poles and zeros on achievable performance described in this chapter pertain to SISO systems. The presence of dynamics coupled through multiple input and output directions make the analysis of MIMO systems more complex. However, by carefully taking these directions into account, one may continue to successfully apply the SISO results with minor modifications to MIMO problems. One such generalization to extend the Bode sensitivity integral to MIMO systems, is the use of the maximum singular value of the sensitivity function in place of the magnitude of the sensitivity function (Zhou et al., 1996).

Bode's classical results suggest a successful control design should have a high control gain at low frequencies (where tracking performance and disturbance rejection is critical) and a low control gain at high frequencies (to prevent excitation of unmodeled dynamics). 


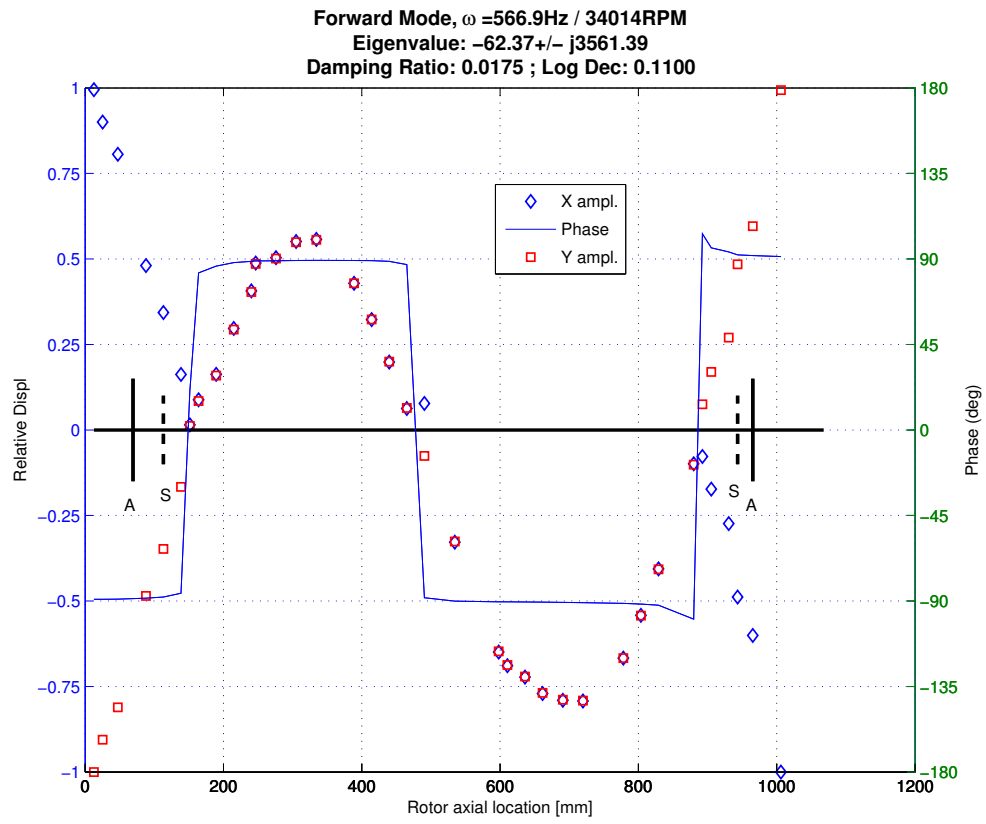

(a) 2D mode shape.

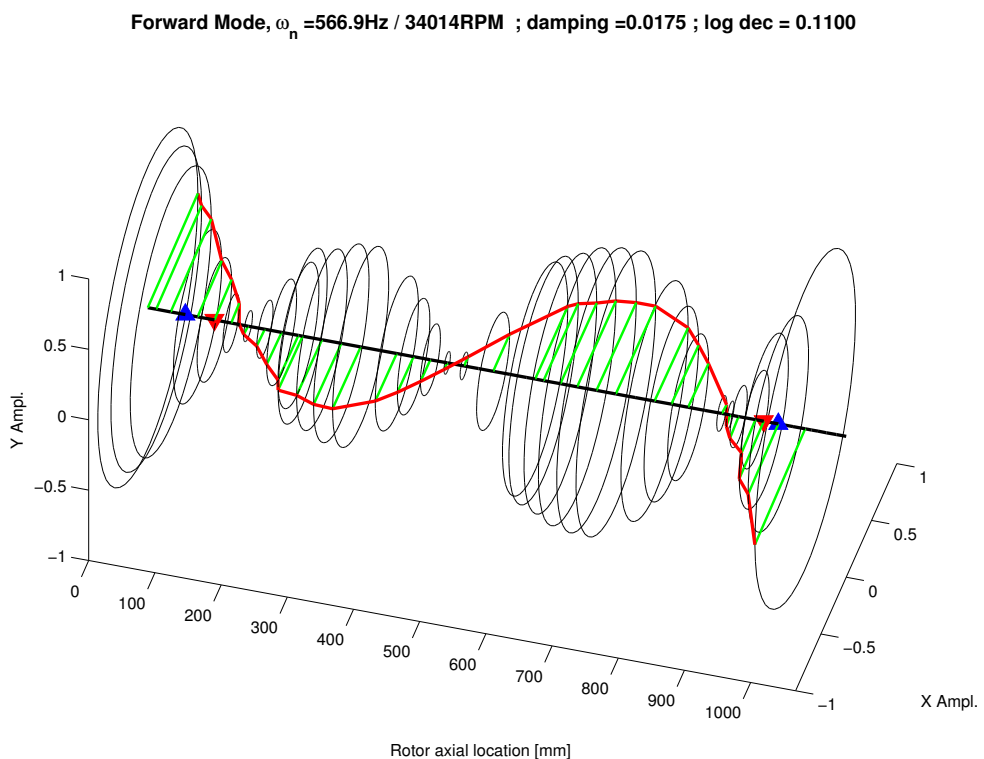

(b) 3D mode shape.

Figure 3.14: Mode shape plots generated during the rotor-AMB system stability analysis. 
The challenge that has hitherto remained unresolved is orchestrating a suitable trade-off between performance and robustness that must occur in the mid-range frequencies to meet the desired criteria (Franchek, 1996). In this chapter the effect of uncertainty in system parameters and the disturbance mechanism on closed-loop stability and performance has not been considered. The development of a suitable uncertainty model will be performed after the complete description and validation of the nominal plant dynamics. 


\section{Chapter 4}

\section{MBTRI Description and Modeling}

This chapter contains details on the MBTRI hardware design and documents the modeling and subsequent steps taken to experiment validate the nominal plant model. An initial design of the MBTRI experiment has been previously documented (Mushi, 2008). However, due to difficulties in commissioning this first design, the rotor and components of the test rig were modified and in some cases completely replaced. A complete description of the current MBTRI experiment is given.

\subsection{Test Rig Design}

\subsubsection{Overview}

The primary goal of the test rig was to emulate relevant rotordynamic characteristics of a single or multistage industrial centrifugal compressor typified by the Frame 25M in Elliott's EDGE product line, for example. The Frame 25M compressor has a nominal speed of 13,100 rpm and rotor length between $1.15 \mathrm{~m}$ and $2.29 \mathrm{~m}$ (Elliott, 2010). The maximum rotor length for the MBTRI was limited by the dimensions of the concrete foundation block in our test cell, while the requirement to have a first bending critical speed close to $12,000 \mathrm{rpm}$ limited the mass and diameter of the rotor. To capture the gyroscopic effects 
of a compressor impeller, a single large disc would be added onto the rotor. A motor with a nominal speed at least $120 \%$ of the first bending critical speed was required. The power and torque requirements of this motor would depend on the mass moment of inertia of the rotating assembly and the rotating power losses due to the bearings.

Four radial AMBs were required for the MBTRI experiment: a pair of radial AMBs at the non-drive end (NDE) and drive end (DE) of the rotor served as supports to stabilize the rotor under the guidance of a feedback control law, and a pair of disturbance AMBs perturbed the rotor with simulated aerodynamic cross-coupled stiffness forces or other static or dynamic loads. The axial position of the support AMBs and the rotor span between them will strongly affect the rotor bending natural frequencies. One exciter was located at the rotor mid-span and the other at the rotor quarterspan to emulate the effects of impellers, balance piston seals and other components in a practical compressor. This combination of four radial AMBs allows for either between-bearing or overhung rotor configurations, representing different compressor designs, to be realized. As four radial AMB stators were available from a previous study (Kasarda et al., 1999), the power amplifier specification was readily established from the calculated maximum static and dynamic bearing loads. The static load requirement for each bearing was at least twice the rotor weight, and the dynamic load requirement was to be estimated from the worst case rotor imbalance and CCS force to be generated.

Use of AMBs mandates the inclusion of backup rolling element bearing adjacent to each support $\mathrm{AMB}$ to protect the rotor and $\mathrm{AMB}$ components in the event of loss of electrical power, failure of one or more amplifiers, or an transient force overload event. A useful design feature was to allow for the removal and replacement of these backup bearings with minimal disruption to other test rig components.

Figure 4.1 illustrates the translation of a sketch of the components of a typical multistage compressor to the functional components required for the MBTRI experiment. 


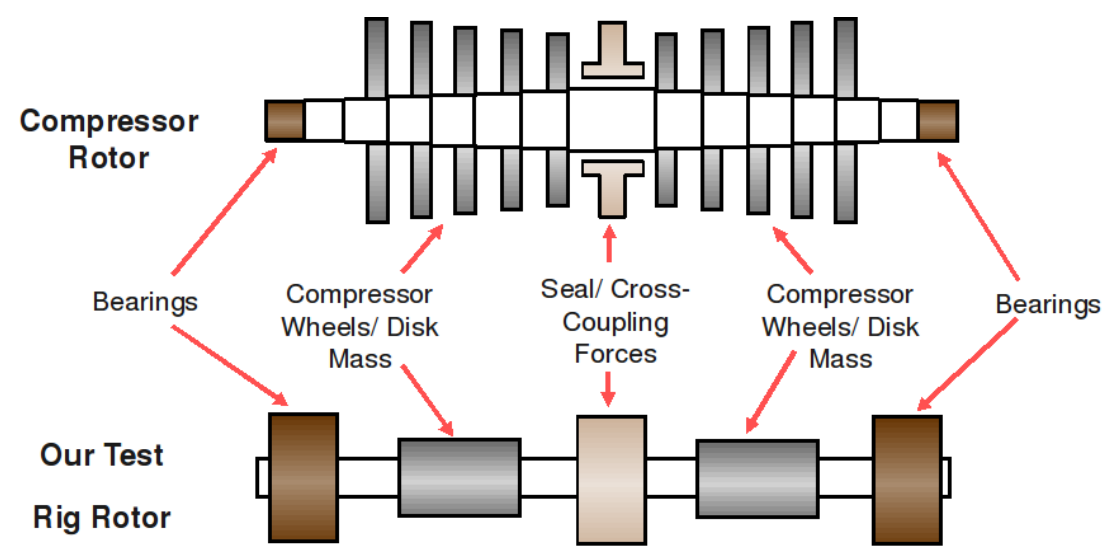

Figure 4.1: A schematic showing the derivation of the test rig rotor design from a back-toback centrifugal compressor.

\subsubsection{Rotor Construction}

The rotor design was a $1068 \mathrm{~mm}$ long solid carbon steel (AISI 1018) shaft with a bearing span of $918 \mathrm{~mm}$ and a predicted bending natural frequency of $220 \mathrm{~Hz}(13,200 \mathrm{rpm})$. The rotor was machined as a single piece with the discs and silicon-iron AMB rotor laminations to be mounted at a later stage (see Figure $4.2 \mathrm{a}$ and $4.2 \mathrm{~b}$ ). The overall weight of the shaft and the added components was $440 \mathrm{~N}$. The hashed regions on the drawing demarcate target areas for the eddy current displacement sensors. In these locations supplemental outer diameter grinding was performed to reduce mechanical runout observed by the sensors. A tolerance of $7 \mu \mathrm{m}$ total indicated runout (TIR) was achieved with respect to the rotation axis $\mathrm{R}$ and support locations $\mathbf{a}$ and $\mathbf{h}$. A minimum surface finish of $0.4 \mu \mathrm{m}$ was attained in these locations. Two discs (labeled $\mathbf{c}$ and $\mathbf{e}$ ) of different diameters were mounted on the rotor to emulate the mass and inertia of an impeller arrangement. Disc c, which imparted a significant gyroscopic character to the rotor, had a length of $25.4 \mathrm{~mm}$, diameter of 241.3 $\mathrm{mm}$ and weighed $82.8 \mathrm{~N}$. While disc e was $101 \mathrm{~mm}$ long $90 \mathrm{~mm}$ in diameter and weighed 27.5 N. The discs also served as balancing planes by drilling several threaded holes into their periphery to accommodate balancing weights. Each disc was mounted with a Class FN1 light drive interference fit to prevent detachment during high speed operation. The design lacked an active thrust magnetic bearing due the absence of significant axial loads. 
To help, the reluctance centering effect of the two radial AMBs acts to attenuate minimal axial disturbances. Disc $\mathbf{c}$ is made from magnetic steel and can serve the additional purpose of a thrust collar, if necessary.

Four laminated silicon-iron journals (b, $\mathbf{d}, \mathbf{f}, \mathbf{g}$ in Figure $4.2 b)$ were mounted with a locational interference fit onto the shaft for attraction by the non-drive end (NDE) and drive end (DE) radial support AMB stators and the two radial disturbance AMB stators. The outer diameter of each lamination stack was varied relative to the inner diameter of the available AMB stators to ensure similar nominal air gaps for all four bearings. The outer surface of the lamination stacks was subject to precision outer diameter grinding to achieve a runout tolerance of $7 \mu \mathrm{m}$.

A pair of NPB 6011 ball bearings ( $55 \mathrm{~mm}$ bore, $90 \mathrm{~mm}$ outer diameter) were used as backup bearings for the support AMBs at locations $\mathbf{a}$ and $\mathbf{h}$ in Figure 4.2b. The rotor rests on this pair of ball bearings when the AMBs are not active. Acetal bushings were used at the mid-span and quarter span AMB locations to protect the bearings in case of severe rotor elastic deflection or failure of the ball bearings. The acetal bushings were vertically split for simple replacement. As shown in Figure 4.3, the shaft, AMBs, and bearing pedestals are mounted on a thick steel plate over a large concrete block to provide a rigid foundation.

\subsubsection{Electrical Components}

Each AMB is driven by four separate analog switchmode power amplifiers. The pair of support AMBs are driven by a total of eight Copley Controls 422 amplifiers operating from a 150V DC supply with a 10 A continuous current rating. The mid-span disturbance AMB is driven by four Copley Controls 413 amplifiers operating from a $75 \mathrm{~V} \mathrm{DC}$ supply and 15 A continuous current rating. The quarter span exciter AMB is driven by four Copley Controls 4212 amplifiers operated from a 75 V DC supply and 6 A continuous current rating. The power bandwidth, small-signal bandwidth and load capacity of the amplifier and AMB pairs are described in more detail in upcoming sections. 

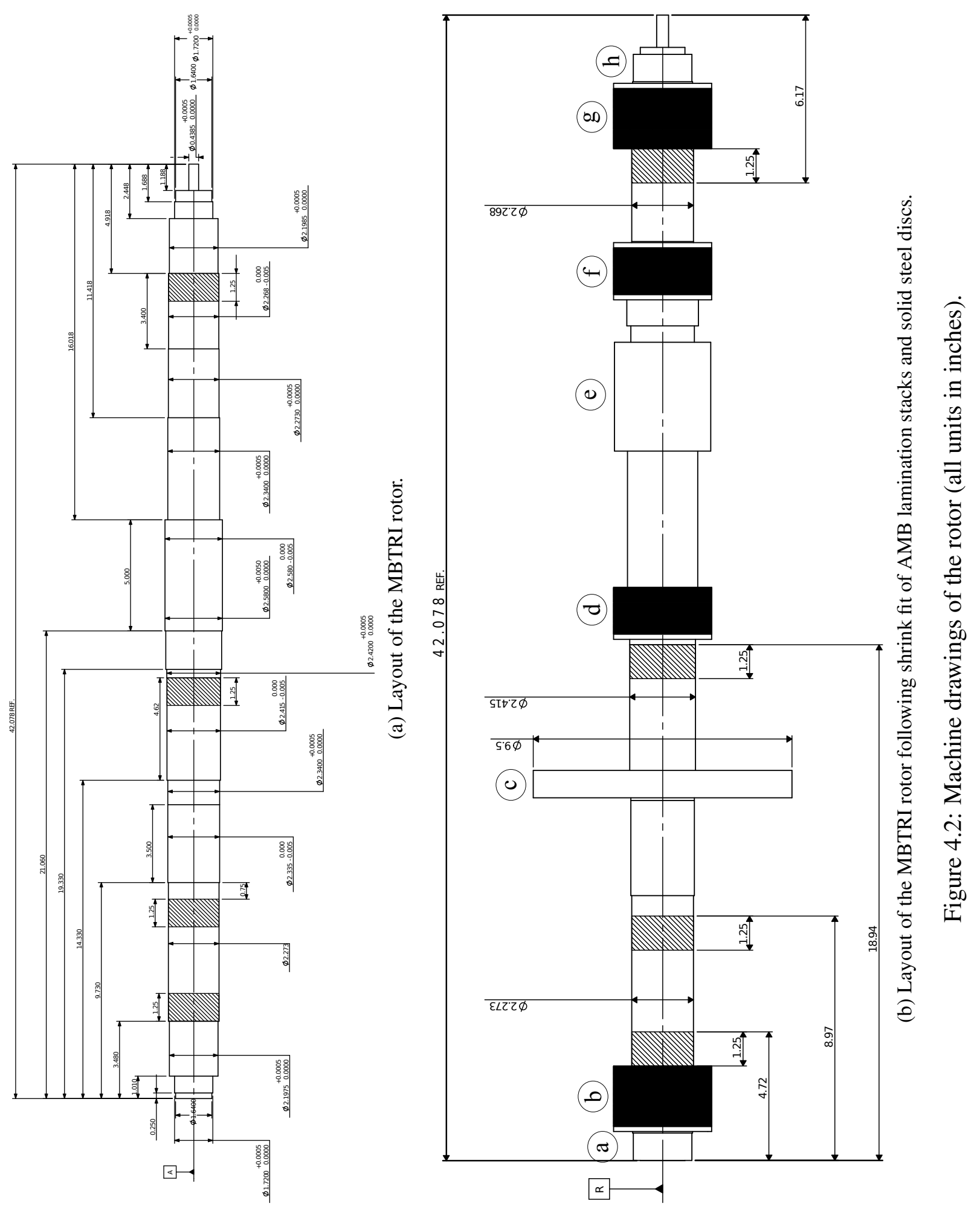
The initial design of the test rig utilized a Toshiba $3.7 \mathrm{~kW}$ inverter-duty AC motor connected to the MBTRI rotor via a trapezoidal tooth timing belt drive with a speed increasing ratio of approximately two. Difficulties in the precision alignment of the side-by-side power transmission system coupled with high vibration levels prevented successful operation of the rotor above 3,000 rpm. An alternative direct drive transmission capable of operating to higher speeds with minimal vibration was sought. The $3.7 \mathrm{~kW}$ Colombo RS-90/2 electric fan cooled, high-speed spindle and accompanying variable frequency drive (VFD) was procured as a suitable replacement. The maximum operating speed of the new motor was $18,000 \mathrm{rpm}$, and a constant motoring torque of $2.2 \mathrm{Nm}$ was available up to this speed. The motor can be configured to run up to $24,000 \mathrm{rpm}$ by altering the base frequency of the VFD from $300 \mathrm{~Hz}$ to $400 \mathrm{~Hz}$, though less torque will be available. The motor is connected to one end of a flexible disc coupling (Rexnord 75CC140140) using a custom shaft extension into the collet nose of the spindle. The other end of the coupling is mated to the MBTRI rotor.

A Kaman eddy current probe measuring system was used to detect rotor motion at the support AMB locations. A pair of Kaman $15 \mathrm{~N}$ probes were setup (four per bearing) in a differential arrangement to measure displacement along each control axis. A pair of Bently Nevada 7200 series eddy current probes were used at the midspan and quarterspan exciter AMB locations. The signals from all eight displacement measurement channels, i.e., $x$ and $y$ at four radial bearings, were fed through custom anti-aliasing filter prior to sampling. Table 4.1 provides a summary of the test rig properties.

The heart of the digital control system was the Innovative Integration M6713 PCI board which is very well suited for real-time servo algorithms. The controller contains a Texas Instruments C6713B 32-bit floating point digital signal processing (DSP) chip, and sixteen channels of simultaneously sampled analog input and output to interface with the sensors and actuators with 16-bit precision, and 32 digital input/output lines. A variety of control algorithms, e.g., PID, LQG, $H_{\infty}$ and $\mu$-synthesis, were implemented on the DSP and 
executed at a sampling rate of $12 \mathrm{kHz}$. A custom graphical user interface was written to interact with the real-time controller. Additional data acquisition channels for real-time vibration analysis were designed around a National Instruments cDAQ-9172 USB chassis in which three NI 9234 modules provided analog input and one NI 9401 module provided digital input/output for event triggering. Data was acquired at a sample rate of $6.4 \mathrm{kHz}$ and an interactive power spectrum analysis was performed to reveal the frequency content of the vibration and control signals.

Table 4.1: Summary of MBTRI experiment properties.

\begin{tabular}{|c|c|c|}
\hline Property & Value & Units \\
\hline \hline Total rotor weight & 440 & $\mathrm{~N}$ \\
\hline Rotor length & 1068 & $\mathrm{~mm}$ \\
\hline Bearing span & 918 & $\mathrm{~mm}$ \\
\hline Average shaft diameter & 57.7 & $\mathrm{~mm}$ \\
\hline Area moment of inertia & $5.43 \times 10^{-7}$ & $\mathrm{~m}^{4}$ \\
\hline Mass moment of inertia & 0.0880 & $\mathrm{~kg} \cdot \mathrm{m}^{2}$ \\
\hline 1st free-free lateral bending mode & 13,200 & $\mathrm{rpm}$ \\
\hline Maximum speed & 18,000 & $\mathrm{rpm}$ \\
\hline Motor power & 3.7 & $\mathrm{~kW}$ \\
\hline AMB radial clearance (average) & 550 & $\mu \mathrm{m}$ \\
\hline Backup bearing clearance & 250 & $\mu \mathrm{m}$ \\
\hline Support AMB stator length & 43.6 & $\mathrm{~mm}$ \\
\hline Support AMB bias current & 4.0 & $\mathrm{~A}$ \\
\hline Support AMB negative stiffness & $0.6-0.9$ & $\mathrm{MN} / \mathrm{m}$ \\
\hline Support AMB current gain & $90-100$ & $\mathrm{~N} / \mathrm{A}$ \\
\hline Power amplifier DC bus & 150 & $\mathrm{~V}$ \\
\hline Controller sampling rate & 12 & $\mathrm{kHz}$ \\
\hline
\end{tabular}



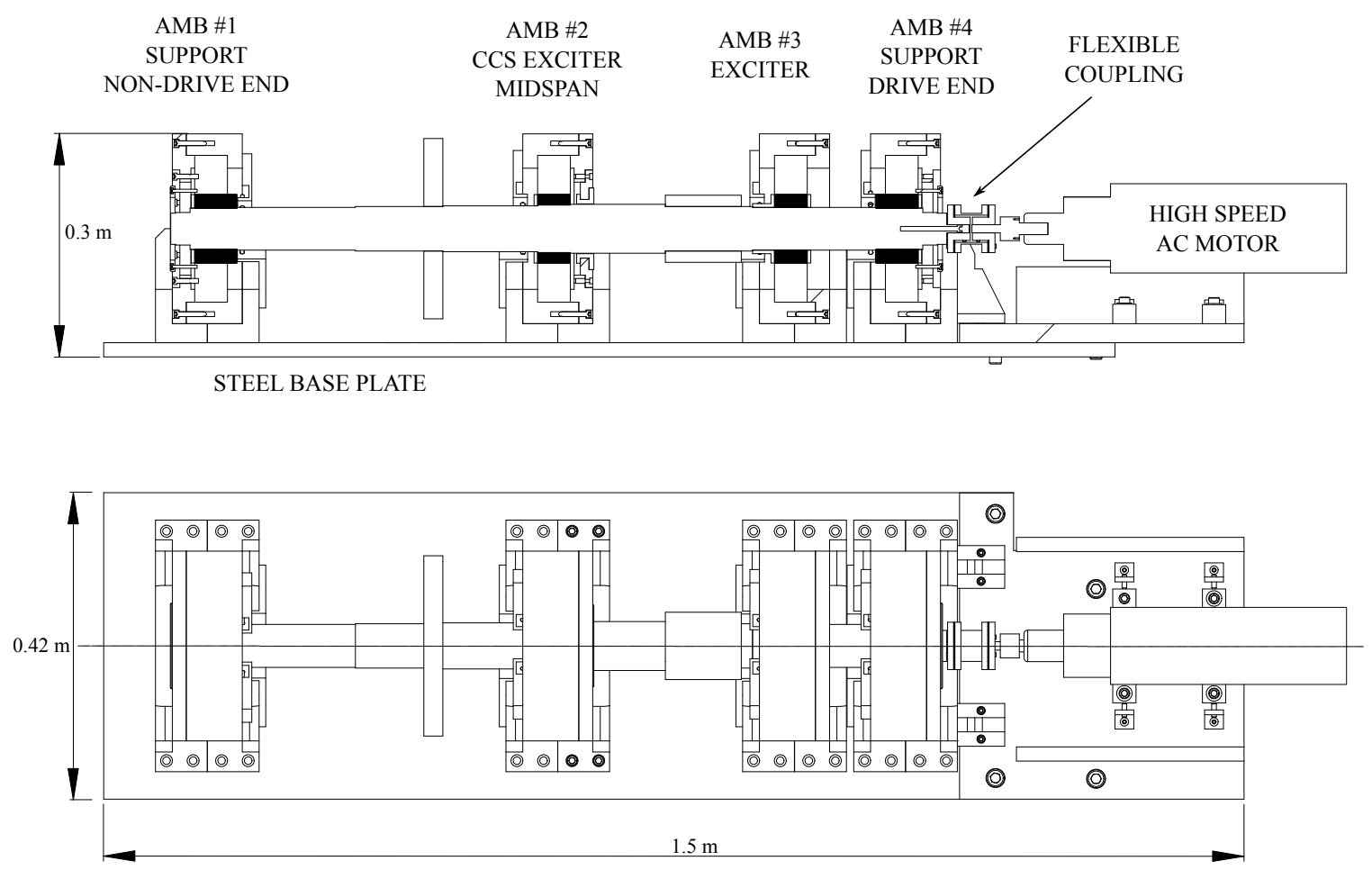

Figure 4.3: Drawing of the test rig assembly.

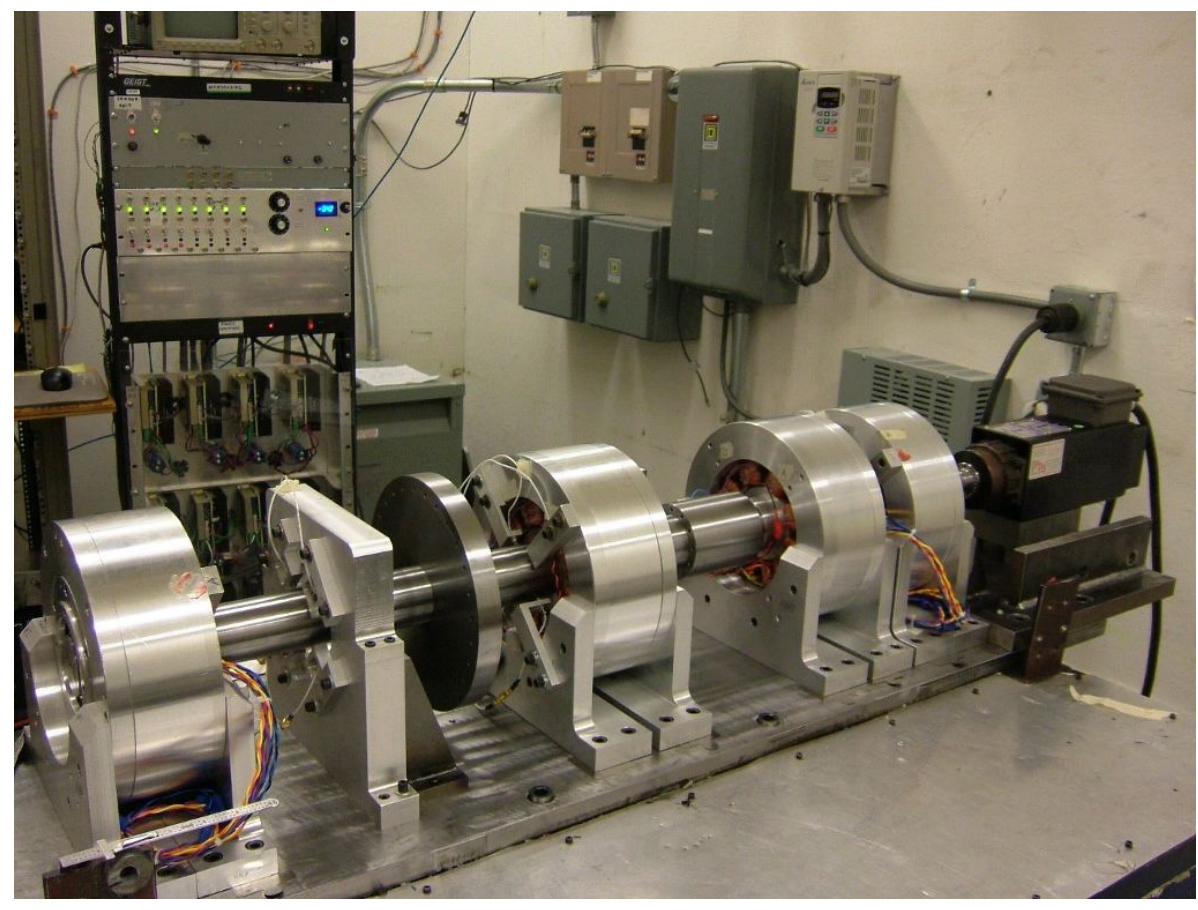

Figure 4.4: Photograph of the completed test rig. 


\subsection{Component Models}

The major components of the digital control system model for a rotor-AMB system are shown in Figure 4.5. The subsequent sections elaborate extensively on first principles modeling of the rotor, magnetic bearings, power amplifiers and additional filters present in the system. For a comprehensive overview of the field, the reader is referred to (Schweitzer \& Maslen, 2009) and references therein.

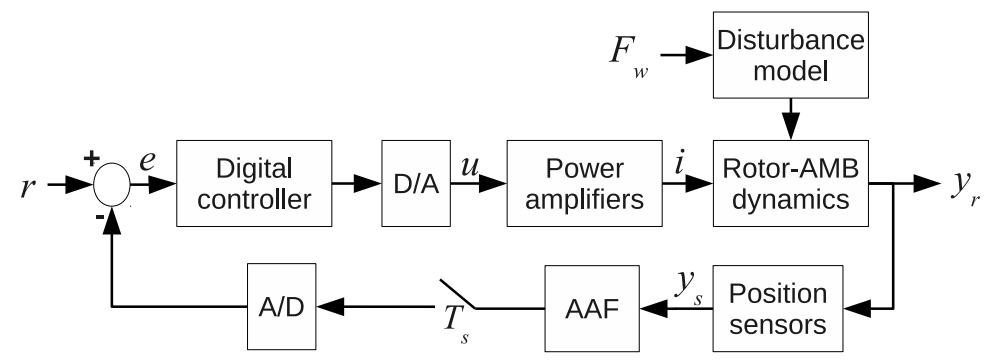

Figure 4.5: A block diagram overview of rotor-AMB control system.

\subsubsection{Rotor Modeling}

A two-dimensional finite-element model of the rotor was obtained by dividing its length into 50 stations for the lateral rotordynamic analysis. The rotor stations were modeled as lumped mass-stiffness elements and the discs and AMB rotor laminations were represented by mass-inertia elements added to a particular station. The bearings were modeled as elastic connections between ground and a particular rotor station. The number of stations in the model was selected based on convergence of the critical speed calculations and to ensure a length/diameter ratio of less than 1 for each station. A custom ROMAC code was used to perform a four degree of freedom per node (4-DOF) lateral analysis. The code generated global entries for the mass $(M)$, internal shaft stiffness $\left(K_{\mathrm{s}}\right)$, internal shaft damping $(D)$, and speed-dependent gyroscopic $(G)$ matrices (Chaudhry \& Sheth, 2008). These matrices are all symmetric and positive definite, except for the gyroscopic matrix which is skew- 
symmetric. The dynamics can be expressed as a second order system

$$
\begin{aligned}
M \ddot{q}+(D+\Omega G) \dot{q}+K_{\mathrm{s}} q & =B_{\mathrm{mag}} F_{\mathrm{mag}}+B_{\mathrm{w}} F_{\mathrm{w}}, \\
y_{\mathrm{r}} & =C q,
\end{aligned}
$$

where the generalized displacement vector $q=\left[x_{1}, \theta_{\mathrm{y} 1}, \ldots, x_{50}, \theta_{\mathrm{y} 50}, y_{1}, \theta_{\mathrm{x} 1}, \ldots, y_{50}, \theta_{\mathrm{x} 50}\right]^{\mathrm{T}}$ contains 200 elements representing translations in the $x$ and $y$ directions, and angular displacements about the $y$ and $x$ axes, denoted as $\theta_{\mathrm{x}}$ and $\theta_{\mathrm{y}}$, respectively. The $4 \times 1$ vector $F_{\text {mag }}$ represents the force provided by the support AMB actuators at the journal locations specified by the matrix $B_{\mathrm{mag}}$, the vector $F_{\mathrm{w}}$ lumps together all external forces acting on the rotor at locations specified by matrix $B_{\mathrm{w}}$, and $\Omega$ is the speed of rotation. Examples of these external forces are rotor weight, destabilizing CCS forces at rotor mid-span, and residual rotor imbalance. The $4 \times 1$ vector $y_{\mathrm{r}}$ represents the rotor displacement at the position sensor locations specified by the coefficient distribution matrix $C$. We do not consider axial motion of the rotor and assume it will not affect the radial displacements.

Equations (4.1) and (4.2) can then be represented in the state space form

$$
\begin{aligned}
\dot{x}_{\mathrm{r}}= & {\left[\begin{array}{cc}
\dot{q} & \ddot{q}
\end{array}\right]^{\mathrm{T}} } \\
= & {\left[\begin{array}{cc}
0 & I \\
-M^{-1} K_{\mathrm{s}} & -M^{-1}(D+\Omega G)
\end{array}\right] x_{\mathrm{r}}+\left[\begin{array}{c}
0 \\
M^{-1} B_{\mathrm{mag}}
\end{array}\right] F_{\mathrm{mag}} } \\
& +\left[\begin{array}{c}
0 \\
M^{-1} B_{\mathrm{w}}
\end{array}\right] F_{\mathrm{W}}:=A_{\mathrm{r}} x_{\mathrm{r}}+B_{1} F_{\mathrm{mag}}+B_{2} F_{\mathrm{w}}, \\
y_{\mathrm{r}}= & {\left[\begin{array}{cc}
C & 0
\end{array}\right] x_{\mathrm{r}}:=C_{\mathrm{r}} x_{\mathrm{r}}, }
\end{aligned}
$$

The system described by Equations (4.3) and (4.4) has 400 states, four control inputs, two exogenous disturbance inputs, and four displacement outputs. Such a large model is unwieldy for control design, and contains a multitude of high order rotor modes beyond 
the AMB controller bandwidth. These modes have small amplitudes and are unlikely to be strongly excited. Modal truncation provides a simple and efficient way to obtain an accurate reduced order model (Bucher, 1985; Losch, 2002). The eigenvalue problem for single plane undamped rotor model is solved at standstill, i.e. $D=0$ and $\Omega G=0$, which completely decouples the $x$ and $y$ dynamics. The mass normalized eigenvector matrix $\Phi$ is partitioned into $\left[\begin{array}{lll}\Phi_{\mathrm{m}} & \mid & \Phi_{s}\end{array}\right]$, where $\Phi_{\mathrm{m}}$ represents the mode shapes of the 5 dominant modes to be retained. The mass and stiffness matrices are simultaneously diagonalized and truncated

$$
\begin{aligned}
& \Phi_{\mathrm{m}}^{\mathrm{T}} M \Phi_{\mathrm{m}}=I \\
& \Phi_{\mathrm{m}}^{\mathrm{T}} K \Phi_{\mathrm{m}}=\Lambda^{2}
\end{aligned}
$$

where $\Lambda$ is a diagonal matrix with entries equal to the natural frequencies of the first five rotor modes in increasing order. The coordinate transformation between modal space and physical space is represented by $q=\Phi_{\mathrm{m}} z$. To better reflect the observed behavior of physical rotors low levels of modal damping $(0.1$ - $0.2 \%)$ were added (Maslen et al., 1996). The resulting modal system matrix for the dynamics in a single plane is

$$
A_{\mathrm{m}}=\left[\begin{array}{cc}
0 & I \\
-\Lambda^{2} & -2 \zeta \Lambda
\end{array}\right]
$$

where the modal damping coefficient $\zeta=0.001$ to 0.002 . Considering the dynamics in two planes, and similarly modally truncating the gyroscopic matrix, i.e., $G_{\mathrm{m}}=\Phi_{\mathrm{m}}^{\mathrm{T}} G \Phi_{\mathrm{m}}$,

$$
\begin{aligned}
& \dot{x}_{\mathrm{m}}=\left[\begin{array}{c}
\dot{z}_{x} \\
\dot{z}_{y}
\end{array}\right]=\left[\begin{array}{cc}
A_{\mathrm{m}} & \Omega G_{\mathrm{m}} \\
-\Omega G_{\mathrm{m}} & A_{\mathrm{m}}
\end{array}\right]\left[\begin{array}{c}
z_{x} \\
z_{y}
\end{array}\right]+\left[\begin{array}{cc}
\Phi_{\mathrm{m}}^{\mathrm{T}} B_{\mathrm{mag}} & \\
& \Phi_{m}^{\mathrm{T}} B_{\mathrm{mag}}
\end{array}\right] F_{\mathrm{mag}}+\left[\begin{array}{cc}
\Phi_{\mathrm{m}}^{\mathrm{T}} B_{\mathrm{w}} & \\
& \Phi_{\mathrm{m}}^{\mathrm{T}} B_{\mathrm{w}}
\end{array}\right] F_{\mathrm{w}}(4.8) \\
& y_{\mathrm{r}}=\left[\begin{array}{cc}
C_{x} \Phi_{\mathrm{m}} & \\
& C_{y} \Phi_{\mathrm{m}}
\end{array}\right]\left[\begin{array}{c}
z_{x} \\
z_{y}
\end{array}\right] \text {. }
\end{aligned}
$$


The absence of damping $D$ in this final system representation comes as little surprise as the majority of the overall damping in rotor-AMB systems comes from the feedback algorithm (an actual AMB supported compressor will have a non-zero $D$ matrix as a result of contributions from the seals). A strong justification for the truncation of the model beyond the third bending mode is the relative magnitude of the Hankel singular values of a system. The distribution of the magnitude of these singular values indicate the internal modes that dominate energy transfer in a system (Bleuler et al., 1994; Skogestad \& Postlethwaite, 2005). The Hankel singular values for the first five rotor modes, in ascending order from $\mathrm{Nc} 1$ to $\mathrm{Nc} 5$, are 10.1, 10.1, 9.35, 9.32 and approximately 0.1 with the remaining higher order modes several orders of magnitude lower (see Figure 4.6). Therefore, truncation of the rotor model beyond the third bending mode is justified as the higher modes have negligible contributions, in an input-output sense, to the system dynamics. Furthermore, as the fourth bending mode (located at $1504 \mathrm{~Hz}$ ) is in the roll-off region of the controller, it was truncated leaving behind the first three bending modes plus the two rigid body modes in the model. The resulting modal state space rotor model has 20 states and is described as

$$
\begin{aligned}
\dot{x}_{\mathrm{m}} & =A_{\mathrm{m}} x_{\mathrm{m}}+B_{1 \mathrm{~m}} F_{\mathrm{mag}}+B_{2 \mathrm{~m}} F_{\mathrm{w}}, \\
y_{\mathrm{r}} & =C_{\mathrm{m}} x_{\mathrm{m}} .
\end{aligned}
$$

\subsubsection{Rotordynamic Analysis}

The primary objectives of rotordynamic analysis are to (Vance et al., 2010):

1. Predict critical speeds of the rotor-bearing system, i.e., speeds at which the rotor's unbalance distribution leads to excitation of a natural frequency,

2. Predict the mode shapes (relative displacements at sensors and actuators) of the rotor,

3. Predict natural frequencies of torsional vibration, 


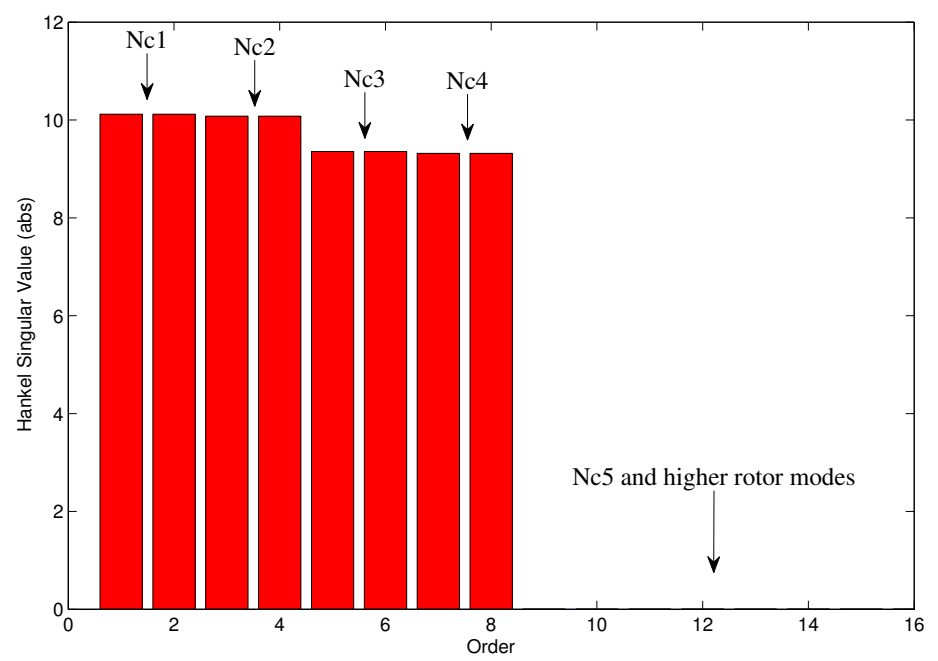

Figure 4.6: Hankel singular values of rotor-AMB model in modal space.

4. Predict amplitude of synchronous vibration response to rotor imbalance,

5. Predict the threshold speeds and destabilizing forces leading the onset of rotordynamic instability,

6. Determine design modifications to suppress the effects of the above resonances during the operation of the rotor.

For the MBTRI experiment, the torsional excitation and resulting vibrations are minimal. As a result torsional vibration is completely ignored. The first five lateral critical speeds of the rotor are calculated for the free-free and $2.5 \mathrm{MN} / \mathrm{m}$ bearing stiffness cases as shown in Table 4.2. A critical speed map with the closed-loop actuator stiffness of an exemplary controller superimposed is given in Figure 4.7. The closed-loop actuator stiffness (in N/m) is computed for the diagonal control axes, i.e., NDEX $\rightarrow$ NDEX. This gives us a good idea of the where the critical speeds may lie early in the design process by superimposing the stiffness plot over the critical speed map (see Figure 4.2). Assuming a controller frequency response $K(s)$ the frequency-dependent closed-loop stiffness of the AMB is

$$
K_{\mathrm{cl}}(s)=K_{\mathrm{i}} G_{\mathrm{a}} G_{\mathrm{s}} K(s)
$$


where $K_{\mathrm{i}}, G_{\mathrm{a}}$ and $G_{\mathrm{s}}$ are the $\mathrm{AMB}$ current gain in N/A, amplifier DC transconductance in A/V, and position sensor sensitivity in V/m, respectively (Allaire et al., 1994). Exclusive use of this closed-loop stiffness plot in determining the critical speeds paints an incomplete picture of the rotordynamics for several reasons:

1. Using MIMO controllers allows the bearing stiffness to be a function of displacement sensed at multiple locations, where the plot only shows the SISO control response used to compute the closed-loop stiffness.

2. The control gains and by extension, the bearing stiffness properties are often anisotropic, i.e., have different gains in vertical and horizontal directions, creating multiple closely spaced critical speeds.

3. Damping, which has a tendency to increase the frequency of the critical speed, has been ignored in this analysis (sources of damping include not only the bearings, but the fluid seals in a practical compressor).

A damped eigenvalue analysis using the rotor model and MIMO controller model provides a more accurate means of computing critical speeds, which takes into account the system damping as well as interactions occurring along multiple axes. However, the utility of the critical speed map remains as the primary means of obtaining approximate critical speed locations as a function of support stiffness.

During the early stages of the design of rotating machinery with flexible rotors, an undamped mode shape analysis is critical to determining whether the axial locations of the position sensors and AMB actuators are suitable to enable control of the modes within the control bandwidth (Schmied et al., 1999). The free-free rotor bending mode shapes Nc3, Nc4 and Nc5 are shown in Figure 4.8, together with the sensor and AMB locations. The inclusion of damping may alter the shape (eigenvector) and frequency (eigenvalue) of the modes shown, hence a complete picture of the rotor mode behavior is not available until the controller has been synthesized. 
At rest $(0 \mathrm{rpm})$ the eigenvalues for the dynamics of the rotor-bearing system in the $x$ and $y$ - directions are identical (assuming symmetric support stiffness). The gyroscopic effect causes the eigenvalues to split into forward and backward components with different natural frequencies as a function of rotor speed, as shown in the Campbell diagram of Figure 4.9. The forward mode is predominantly excited by rotor imbalance at speeds where the $1 \mathrm{X}$ line intersects the forward mode (Ehrich, 2004). The $2 \mathrm{X}$ line is often included in the diagram since harmonics arising from drive-line misalignment, the motor electronics, or sensor runout may be present during operation.

The control law, which has yet to be designed, provides the bulk of the damping in this rotor-bearing system. As a result further rotordynamic analysis to predict the unbalance response and rotor stability (damped mode shapes) will have to wait until the controller was been designed (see Chapter 5.7).

Rotordynamic instability predominantly affects the lowest natural frequency of the rotor-bearing system, i.e., $\mathrm{Nc1}$. The natural frequency of $\mathrm{Nc} 1$ is approximately $50 \mathrm{~Hz}$ depending on support stiffness levels and running speed. Using Equations (3.9)-(3.11), we can estimate the optimum damping that will maximize the stability threshold.

From Equation 3.9, effective shaft stiffness $k_{\mathrm{s}}$ is $4.28 \times 10^{6} \mathrm{~N} / \mathrm{m}$. Assuming a typical support (bearing) stiffness $k_{\mathrm{b}}$ of $2 \mathrm{MN} / \mathrm{m}$ (in vicinity of $\mathrm{Nc} 1$ ), the stiffness ratio $K_{\mathrm{r}}$ is 0.70 . The rigid bearing critical speed $\omega_{\text {cr }}$ is $735 \mathrm{rad} / \mathrm{s}$ or 7,030 rpm (see Figure 4.7), the modal rotor mass $M_{m}$ is approximately half the rotor mass or $22.3 \mathrm{~kg}$. From Equation (3.10), the estimated optimal damping $\hat{c}$ is $14,100 \mathrm{~N} \mathrm{~s} / \mathrm{m}$, which results in a maximum allowable aerodynamic excitation of $3540 \mathrm{~N} / \mathrm{mm}$ from Equation (3.11).

Table 4.2: The first six rotor critical speeds.

\begin{tabular}{|c|c|c|}
\hline Mode & Free-Free & $2.5 \mathrm{MN} / \mathrm{m}$ support stiffness \\
\hline \hline $\mathrm{Nc} 1$ & 0 & $52.2 \mathrm{~Hz}(3,130 \mathrm{rpm})$ \\
\hline $\mathrm{Nc} 2$ & 0 & $88.8 \mathrm{~Hz}(5,327 \mathrm{rpm})$ \\
\hline $\mathrm{Nc} 3$ & $224 \mathrm{~Hz}(13,433 \mathrm{rpm})$ & $243 \mathrm{~Hz}(14,596 \mathrm{rpm})$ \\
\hline Nc4 & $549 \mathrm{~Hz}(32,915 \mathrm{rpm})$ & $552 \mathrm{~Hz}(33,110 \mathrm{rpm})$ \\
\hline Nc5 & $982 \mathrm{~Hz}(58,920 \mathrm{rpm})$ & $982 \mathrm{~Hz}(58,920 \mathrm{rpm})$ \\
\hline
\end{tabular}




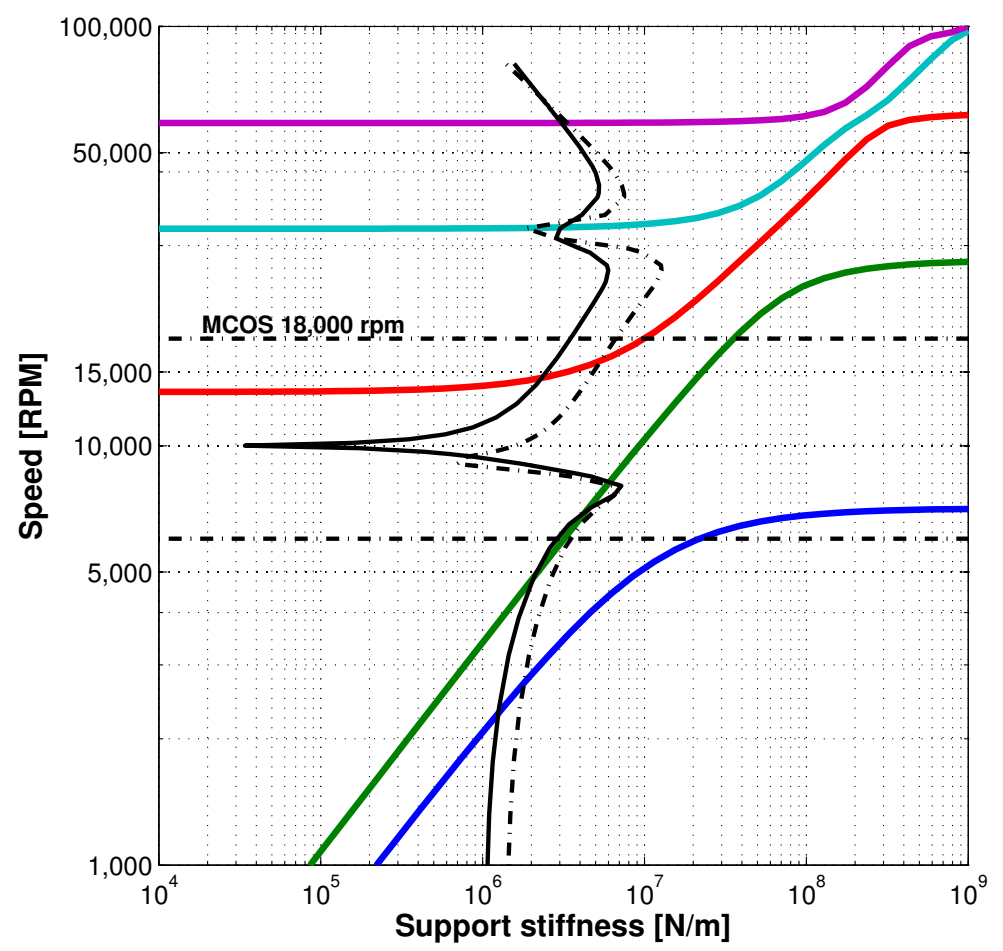

Figure 4.7: Undamped critical speed map showing eigenvalues as a function of support stiffness. The closed-loop actuator stiffness for an exemplary controller is overlaid. 


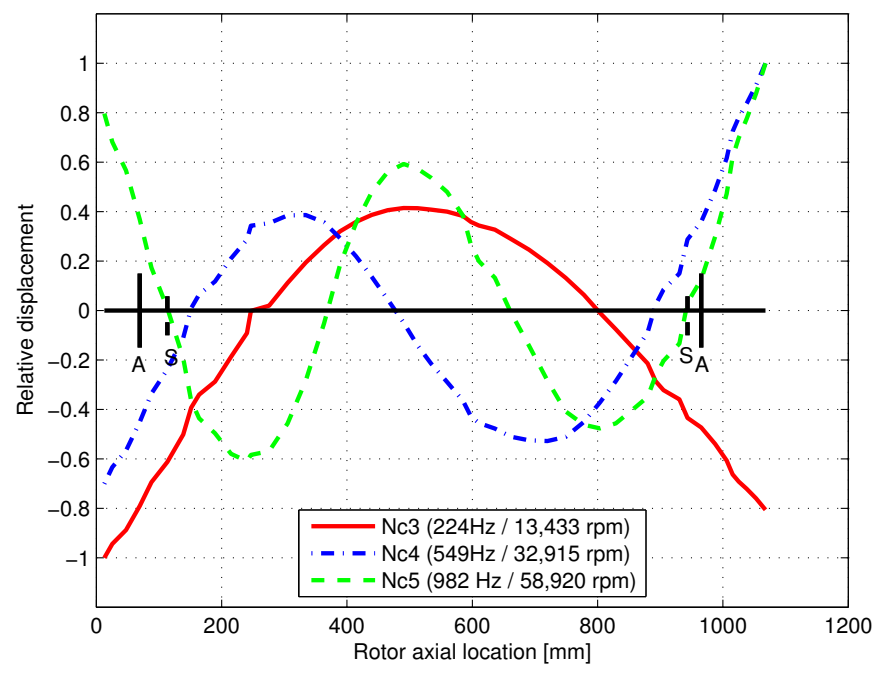

Figure 4.8: Free-free rotor bending mode shapes shown with respect to the sensor and actuator locations.

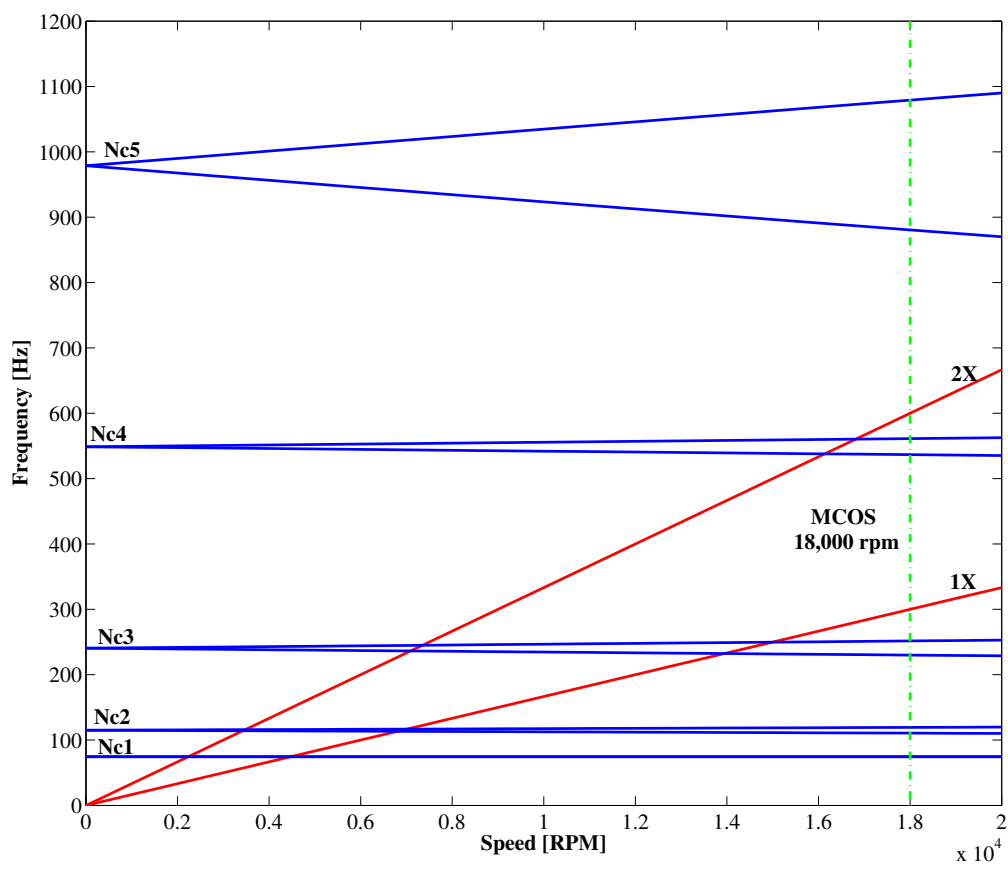

Figure 4.9: Campbell diagram with $5 \mathrm{MN} / \mathrm{m}$ support stiffness showing the variation of rotor eigenvalues with operating speed. 


\subsubsection{Actuator Modeling}

\subsubsection{AMB Specifications}

The support AMB stators are 16-pole heteropolar bearings with laminated 26 gauge M-15 silicon-iron stators, while the disturbance AMBs are 8-pole stators of the same composition. A single control quadrant consists of a series connection of four adjacent poles (or two in the case of the 8-pole stators). The four control quadrants of the support AMBs, shown in different colors in Figure 4.16a, consist of series connected windings 1A-1B-1C1D, 2A-2B-2C-2D, 3A-3B-3C-3D, and 4A-4B-4C-4D. Each disturbance AMB (see Figure 4.16b) consists of series connected windings 1A-1B, 2A-2B, 3A-3B, 4A-4B. Each quadrant formed one leg of a center grounded star configuration, requiring a total of 4 power amplifiers per radial bearing. A conventional N-S-S-N magnetic polarity scheme, indicated by the direction of current flow in the above figures, was used for each quadrant. A single control channel consists of a single pair of opposing quadrants. The orientation of the quadrants, offset $45^{\circ}$ from vertical, allows the rotor weight to be distributed equally between the two control channels. This ensures the $x$ and $y$ dynamics will be nearly identical. Table 4.3 summarizes the physical properties of both types of radial AMB actuators used in the test rig. 
Table 4.3: Support and disturbance AMB specifications.

\begin{tabular}{|c|c|c|c|c|c|}
\hline Property & NDE & $\mathrm{DE}$ & MID & QTR & Units \\
\hline Number of poles, $n$ & \multicolumn{2}{|c|}{$\overline{16}$} & \multicolumn{2}{|c|}{8} & - \\
\hline Stator axial length, $l$ & \multicolumn{2}{|c|}{43.6} & \multicolumn{2}{|c|}{43.6} & $\mathrm{~mm}$ \\
\hline Stator outer diameter, $D_{\mathrm{o}}$ & \multicolumn{2}{|c|}{196} & \multicolumn{2}{|c|}{196} & $\mathrm{~mm}$ \\
\hline Stator inner diameter, $D_{\mathrm{i}}$ & 92.412 & 92.424 & 92.418 & 92.358 & $\mathrm{~mm}$ \\
\hline Rotor lamination diameter, $D$ & 91.377 & 91.237 & 91.269 & 91.377 & $\mathrm{~mm}$ \\
\hline Projected pole area, $A_{\mathrm{p}} \dagger$ & \multicolumn{2}{|c|}{348} & \multicolumn{2}{|c|}{700} & $\mathrm{~mm}^{2}$ \\
\hline Back iron thickness, $T_{\mathrm{b}}$ & \multicolumn{2}{|c|}{0.0171} & \multicolumn{2}{|c|}{0.0201} & $\mathrm{~mm}$ \\
\hline Nominal air gap, $g_{0}$ & 0.518 & 0.593 & 0.575 & 0.491 & $\mathrm{~mm}$ \\
\hline Copper turns, $N$ & \multicolumn{2}{|c|}{48} & \multicolumn{2}{|c|}{94} & - \\
\hline Copper wire gauge & \multicolumn{2}{|c|}{$\# 18$} & \multicolumn{2}{|c|}{$\# 18$} & AWG \\
\hline Quadrant inductance, $L_{\mathrm{W}}$ & \multicolumn{2}{|c|}{12} & \multicolumn{2}{|c|}{36} & $\mathrm{mH}$ \\
\hline Quadrant resistance, $R_{\mathrm{W}}$ & \multicolumn{2}{|c|}{0.35} & \multicolumn{2}{|c|}{0.34} & $\Omega$ \\
\hline Stator weight & \multicolumn{2}{|c|}{35.6} & \multicolumn{2}{|c|}{53.4} & $\mathrm{~N}$ \\
\hline
\end{tabular}

$\dagger$ For a single pole in a quadrant

\subsubsection{Linearized AMB Model}

The force generated by an AMB has a nonlinear dependence on the rotor-stator air gap and current flowing through the stator windings. The following equation expresses the net force in the $x$-direction produced by, for instance, currents $I_{1}$ and $I_{3}$ flowing in quadrants 1 and 3 of the support AMB

$$
F_{\text {mag }, x}=\alpha \mu_{0} N^{2} A_{\mathrm{p}}\left(\frac{I_{1}^{2}}{x_{1}^{2}}-\frac{I_{3}^{2}}{x_{3}^{2}}\right),
$$

where the constant $\alpha$ is the product of the number of pole pairs per quadrant and cosine of the pole face angle $\left(22.5^{\circ}\right.$ for support $\mathrm{AMB}$ and $45^{\circ}$ for disturbance AMBs), and $x_{1}$ and $x_{3}$ are the distances of the rotor from the face of each quadrant. $\alpha=1.85$ for the support AMBs and $\alpha=0.707$ for the disturbance AMBs. By replacing $I_{1}$ and $I_{3}$ with the sum and difference of a constant bias current $I_{\mathrm{b}}$ and variable perturbation current $i_{\mathrm{px} 1}$, and then limiting the rotor displacement to small motions $x_{\mathrm{px} 1}$ about its nominal center, we arrive at an approximate linear relationship for the force delivered by the opposing quadrants

$$
F_{\mathrm{mag}, \mathrm{x} 1} \approx 4 \frac{k I_{\mathrm{b}}}{g_{0}^{2}} i_{\mathrm{px} 1}+4 \frac{k I_{\mathrm{b}}^{2}}{g_{0}^{3}} x_{\mathrm{px} 1}=K_{\mathrm{i}} i_{\mathrm{px} 1}-K_{\mathrm{x}} x_{\mathrm{px} 1}
$$


where $k=\alpha \mu_{0} N^{2} A_{\mathrm{p}}, K_{\mathrm{x}}$ and $K_{\mathrm{i}}$ represent the open-loop stiffness and current gain of the AMB actuator (Schweitzer \& Maslen, 2009). This magnetic circuit model assumes infinite permeability of the silicon-iron, and negligible flux leakage (Meeker et al., 1995). In accordance with Equation (4.3), the $4 \times 1$ vector of the AMB force acting on the rotor in the $x$ and $y$-directions at the two support bearing planes is constructed as $F_{\text {mag }}=$ $\left[F_{\mathrm{mag}, \mathrm{x} 1} F_{\mathrm{mag}, \mathrm{x} 2} F_{\mathrm{mag}, \mathrm{y} 1} F_{\mathrm{mag}, \mathrm{y} 2}\right]^{\mathrm{T}}$. The perturbation current vector $i_{\mathrm{p}}=\left[\begin{array}{ll}i_{\mathrm{p} x 1} i_{\mathrm{px} 2} i_{\mathrm{py} 1} i_{\mathrm{py} 2}\end{array}\right]^{\mathrm{T}}$, while the rotor displacements from the equilibrium position measured at the bearing planes are given by the $4 \times 1$ vector $x_{\mathrm{p}}=\left[\begin{array}{llll}x_{\mathrm{px} 1} & x_{\mathrm{px} 2} & x_{\mathrm{py} 1} & x_{\mathrm{py} 2}\end{array}\right]^{\mathrm{T}}$, defined such that $x_{\mathrm{p}}=\left[\begin{array}{ll}B_{\mathrm{mag}} & 0\end{array}\right] x_{\mathrm{r}}$. Table 4.4 summarizes the linear AMB actuator properties derived from the aforementioned magnetic circuit model.

Table 4.4: Linearized actuator properties for support and disturbance AMBs.

\begin{tabular}{|c|c|c|c|c|}
\hline Property & \multicolumn{2}{|c|}{ Support AMB } & Exciter AMB & Units \\
\hline & NDE & DE & MID & \\
\hline \hline Bias current, $I_{\mathrm{b}}$ & 4.0 & 4.0 & 1.0 & $\mathrm{~A}$ \\
\hline Estimated air gap flux density & 0.55 & 0.5 & 0.27 & $\mathrm{~T}$ \\
\hline Current gain, $K_{\mathrm{i}}$ & 130 & 100 & 94 & N/A \\
\hline Negative stiffness, $K_{\mathrm{x}}$ & 900 & 600 & 165 & $\mathrm{kN} / \mathrm{m}$ \\
\hline
\end{tabular}

By combining the rotor model from (4.10) with the linear AMB actuator model we arrive at the description

$$
\begin{aligned}
\dot{x}_{\mathrm{m}} & =A_{\mathrm{m}} x_{\mathrm{m}}+B_{1 \mathrm{~m}}\left(-K_{\mathrm{x}}\left[\begin{array}{ll}
B_{\mathrm{mag}} & 0
\end{array}\right] x_{\mathrm{m}}+K_{\mathrm{i}} i_{\mathrm{p}}\right)+B_{2 \mathrm{~m}} F_{\mathrm{w}} \\
& =\left(A_{\mathrm{m}} x_{\mathrm{m}}-B_{1 \mathrm{~m}} K_{\mathrm{x}}\left[\begin{array}{ll}
B_{\mathrm{mag}} & 0
\end{array}\right]\right) x_{\mathrm{m}}+B_{1 \mathrm{~m}} K_{\mathrm{i}} i_{\mathrm{p}}+B_{2 \mathrm{~m}} F_{\mathrm{w}} \\
& :=\hat{A}_{\mathrm{m}} x_{\mathrm{m}}+B_{1 \mathrm{~m}} K_{\mathrm{i}} i_{\mathrm{p}}+B_{2 \mathrm{~m}} F_{\mathrm{w}} . \\
y_{\mathrm{r}} & =C x_{\mathrm{m}} .
\end{aligned}
$$

\subsubsection{Actuator Capacity}

The linear magnetic circuit model is useful for the initial development of linearized AMB parameters. However, the computation of the maximum static and dynamic loads of the 
$\mathrm{AMB}$ violates the assumptions in the ideal magnetic circuit model requiring a finite element based analysis. A two-dimensional finite element magnetostatic analysis of the rotor and stator geometry using the program FEMM (Meeker, 2009) was carried out. The error between Equation (4.13) and the peak static force calculated by the field solution based on Maxwell's stress tensor for the NDE support AMB is shown as function of position and current in Figure 4.10. During controller operation the rotor position is typically restricted to within than $0.05 \mathrm{~mm}$ of the center of the air gap. A linearization error of less than 5 percent suggests the actuators are linear across the full range of perturbation currents. However, as the rotor moves away from the center and perturbation current increases to the maximum, the linear approximation under-predicted the actual force produced by up to $35 \%$.

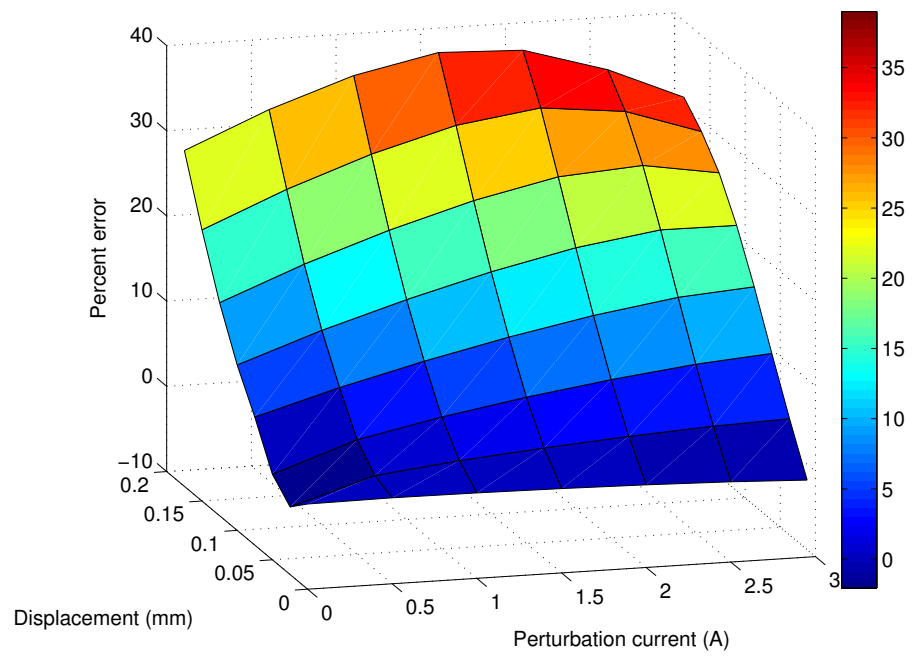

Figure 4.10: Plot of the difference between actuator force outputs determined using a linear magnetic circuit model and a two-dimensional finite element magnetostatic analysis for the NDE AMB.

An finite element analysis (FEA) of the equilibrium state of the support AMB actuator was performed with the rotor centered in the air gap and total current equal to the bias current $(4 \mathrm{~A})$ flowing through all the four quadrants. Figure 4.11 shows the magnetic field lines passing from the NDE AMB stator, through the air gap, through the rotor laminations and back into the stator. There is minimal cross-talk between adjacent poles and more importantly adjacent quadrants. This is important for the control algorithm assumes the 
only coupling between axes takes place through the rotor model. Figure 4.12 illustrates the flux density through a section of the AMB under the equilibrium conditions. Only minute regions of the stator are showing flux densities exceeding the knee flux of the material (1.2 T). The air gap and stator legs have flux densities between 0.4 and $0.6 \mathrm{~T}$, indicating that operation in the vicinity of this equilibrium will be almost linear.

To calculate the maximum static load of the actuator with the rotor centered, the top two quadrants of the NDE AMB are supplied with $8 \mathrm{~A}\left(I_{b}=4 \mathrm{~A}\right.$ and $\left.i_{p}=4 \mathrm{~A}\right)$, whilst the bottom two quadrants are turned off. Figure 4.13 shows the resulting magnetic flux lines, and Figure 4.14 shows the resulting magnetic flux density. The air gap has a flux density between 0.8 and $1.0 \mathrm{~T}$ indicating that perhaps more load may be carried. However, the stator legs have flux densities which in places exceed $1.2 \mathrm{~T}$ suggesting that the magnetic material is beginning to saturate. Linear operation close to this point is not possible. The maximum static force delivered by the NDE and DE support AMBs in this condition is indicated in Table 4.5, along with the estimated air gap flux density. The combined static force capacity of the support AMBs is $1336 \mathrm{~N}$, which is approximately three times the weight of the rotor.
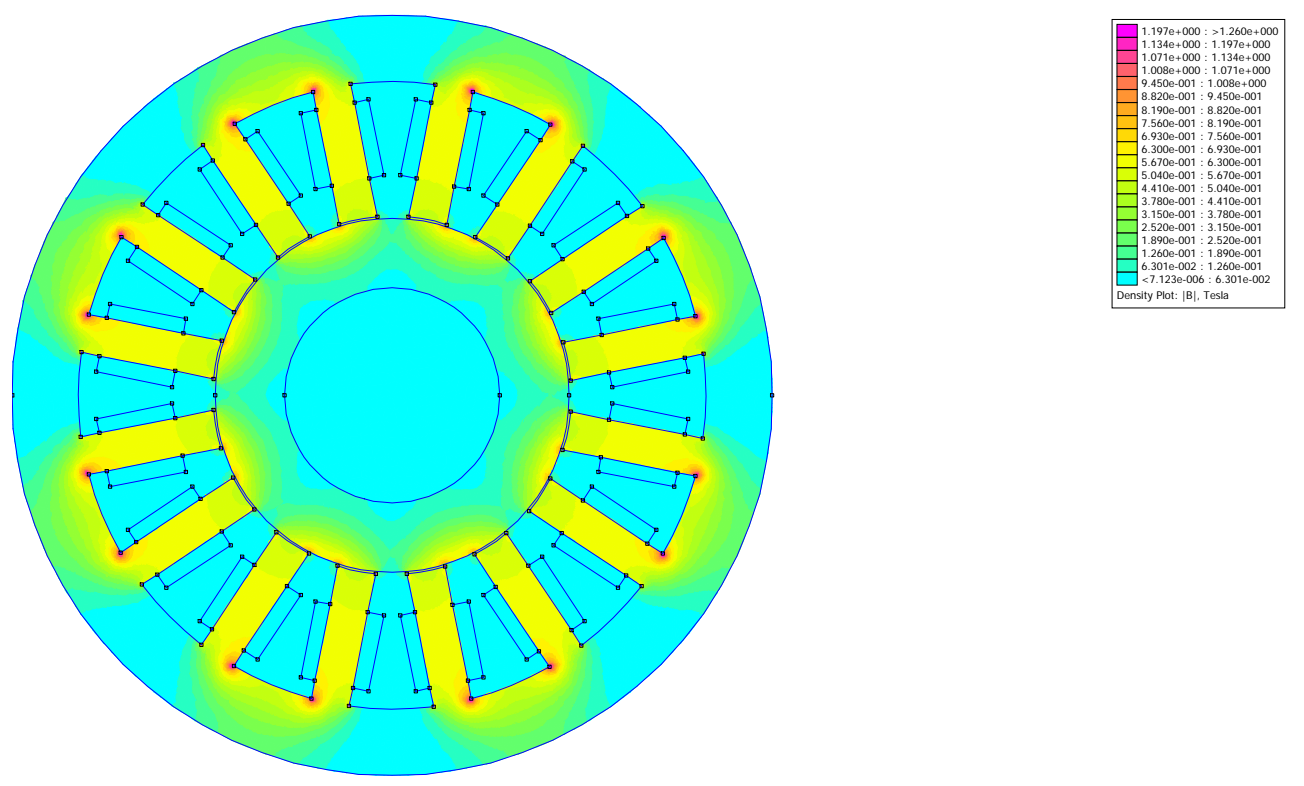

Figure 4.12: Equilibrium flux density in NDE AMB rotor, stator and air gap when only bias current $(4 \mathrm{~A})$ is flowing through all four quadrants. 


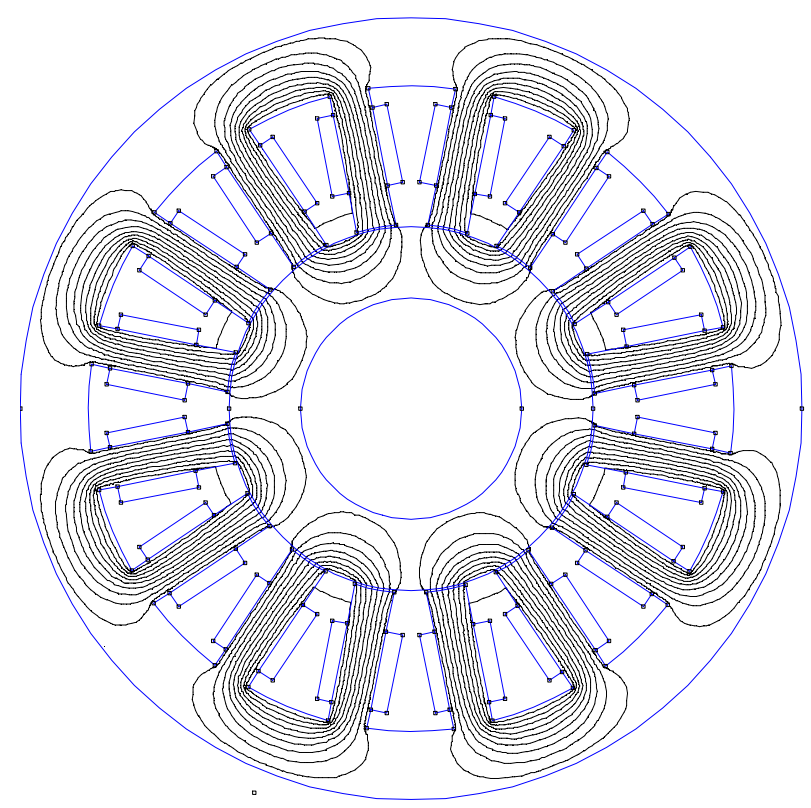

Figure 4.11: Equilibrium field pattern developed in the NDE AMB stator and rotor when only the bias current (4 A) is flowing through each quadrant, and the rotor is centered in the air gap.

Table 4.5: Maximum static force calculated for the support and disturbance AMBs.

\begin{tabular}{|c|c|c|}
\hline Property & \multicolumn{2}{|c|}{ Support AMB } \\
\hline & NDE & DE \\
\hline \hline Maximum current, $I_{\max }$ & $8 \mathrm{~A}$ & $8 \mathrm{~A}$ \\
\hline Estimated air gap flux density & $0.9 \mathrm{~T}$ & $0.77 \mathrm{~T}$ \\
\hline Maximum static force, $F_{\max }$ & $752 \mathrm{~N}$ & $584 \mathrm{~N}$ \\
\hline
\end{tabular}




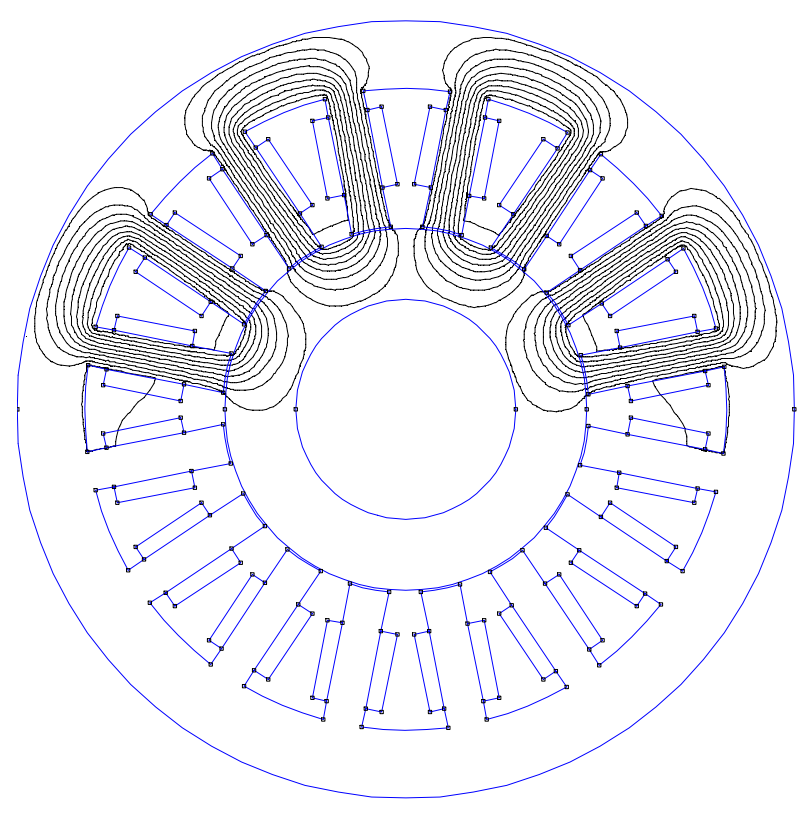

Figure 4.13: Field pattern developed in the NDE AMB stator and rotor with $I_{b}=4 \mathrm{~A}$ and $i_{p}=4 \mathrm{~A}$ through the top quadrants, and the rotor is centered in the air gap.
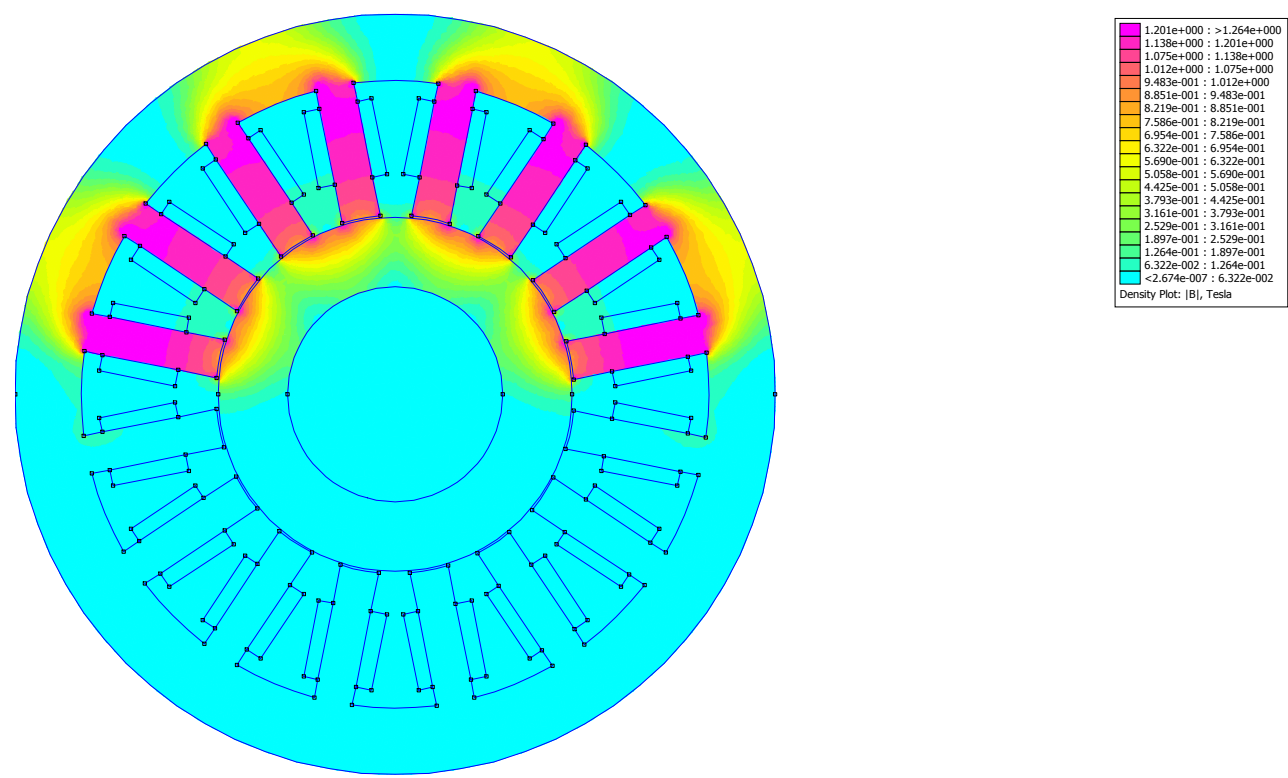

Figure 4.14: Flux density in NDE AMB rotor, stator and air gap computed using 2-D finite elements. The top two quadrants are driven at maximum current $\left(I_{b}=4 \mathrm{~A}\right.$ and $\left.i_{p}=4 \mathrm{~A}\right)$ to calculate the static load capacity at this operating point.

As explained in the previous chapter, the dynamic force capacity of an AMB actuator is a function of several physical parameters, such as the air gap, bias current, existing static bearing load, maximum bearing current, amplifier bus voltage, and the magnetic properties 
of the materials. At higher frequencies (typically one or multiples of the running speed) the AMB winding inductance can generate a back-EMF large enough to begin to overcome the amplifier bus voltage. This leads to a reduction in the dynamic force output of the actuator. The frequency at which this gain roll-off begins is called the knee frequency or power bandwidth $\omega_{\mathrm{pb}}$, and for a well matched combination of power amplifier and AMB actuator $\omega_{\mathrm{pb}}$ should be above the maximum operating speed of the rotor (Maslen et al., 1996; Swanson et al., 2008). Figure 4.15 shows the dynamic force capacity computed for the NDE and DE AMBs assuming a nominal bias current of $4 \mathrm{~A}$ and static load (rotor weight) shared equally between the bearings. The power bandwidths of each bearing are indicated in Table 4.6.

Table 4.6: Dynamic characteristics of the NDE and DE AMBs.

\begin{tabular}{|c|c|c|}
\hline & NDE & DE \\
\hline \hline Low frequency dynamic capacity & $532 \mathrm{~N}$ & $364 \mathrm{~N}$ \\
\hline Power bandwidth, $\omega_{\mathrm{pb}}$ & $486 \mathrm{~Hz}$ & $527 \mathrm{~Hz}$ \\
\hline
\end{tabular}

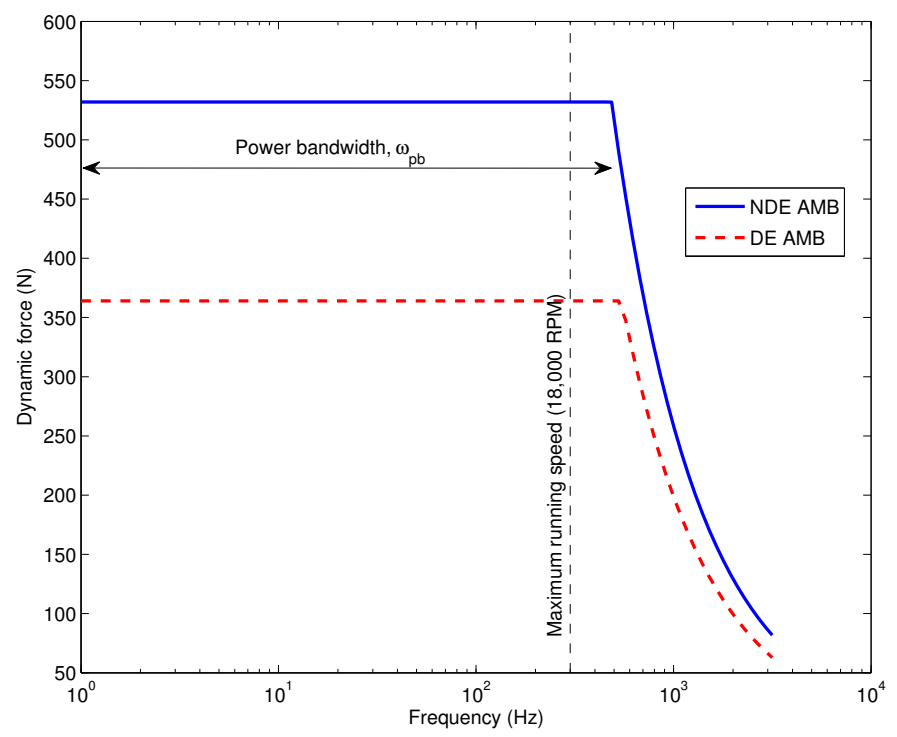

Figure 4.15: Dynamic force capacity as a function of frequency for the NDE and DE AMBs. 

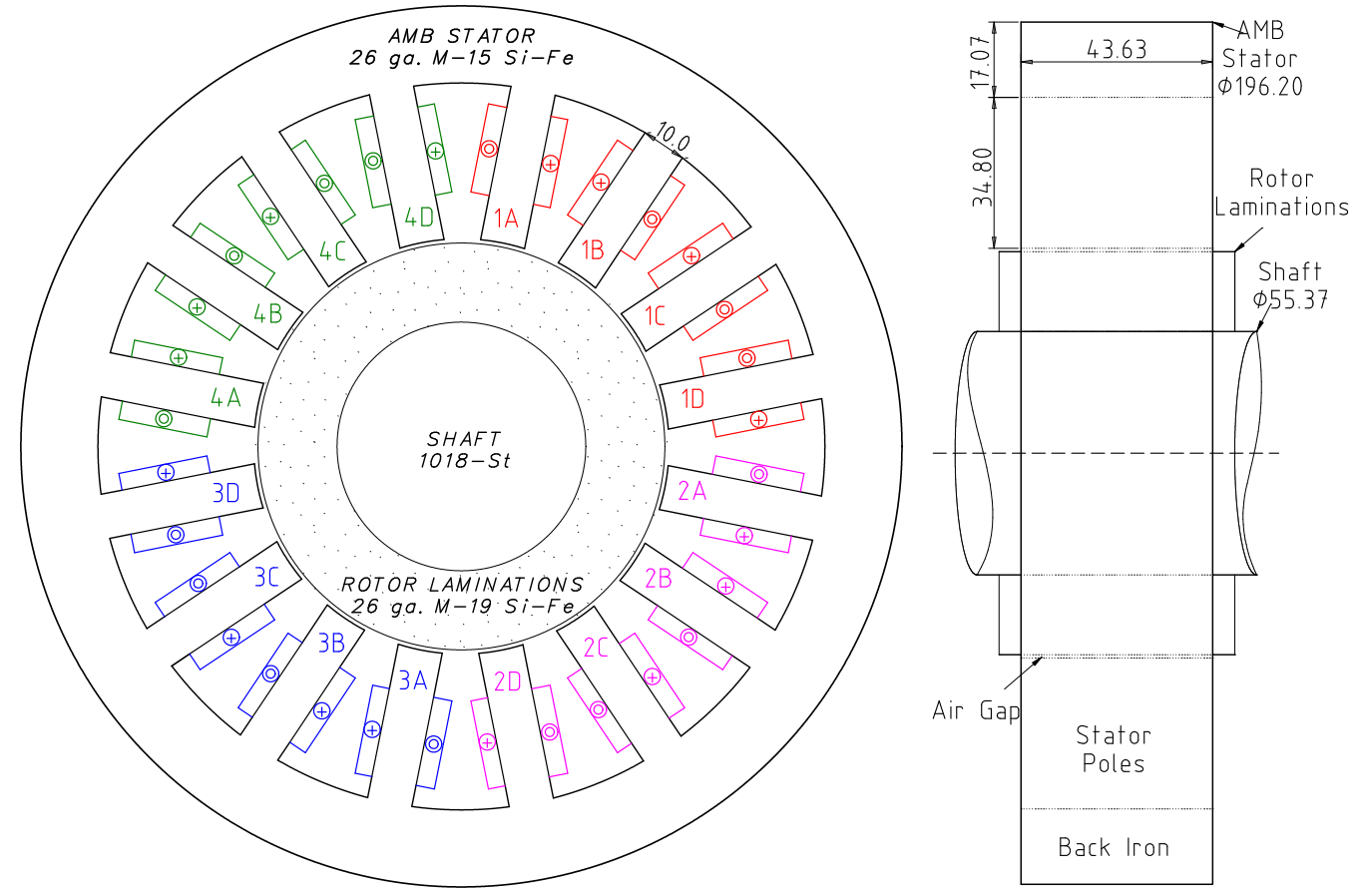

(a) Support AMB.
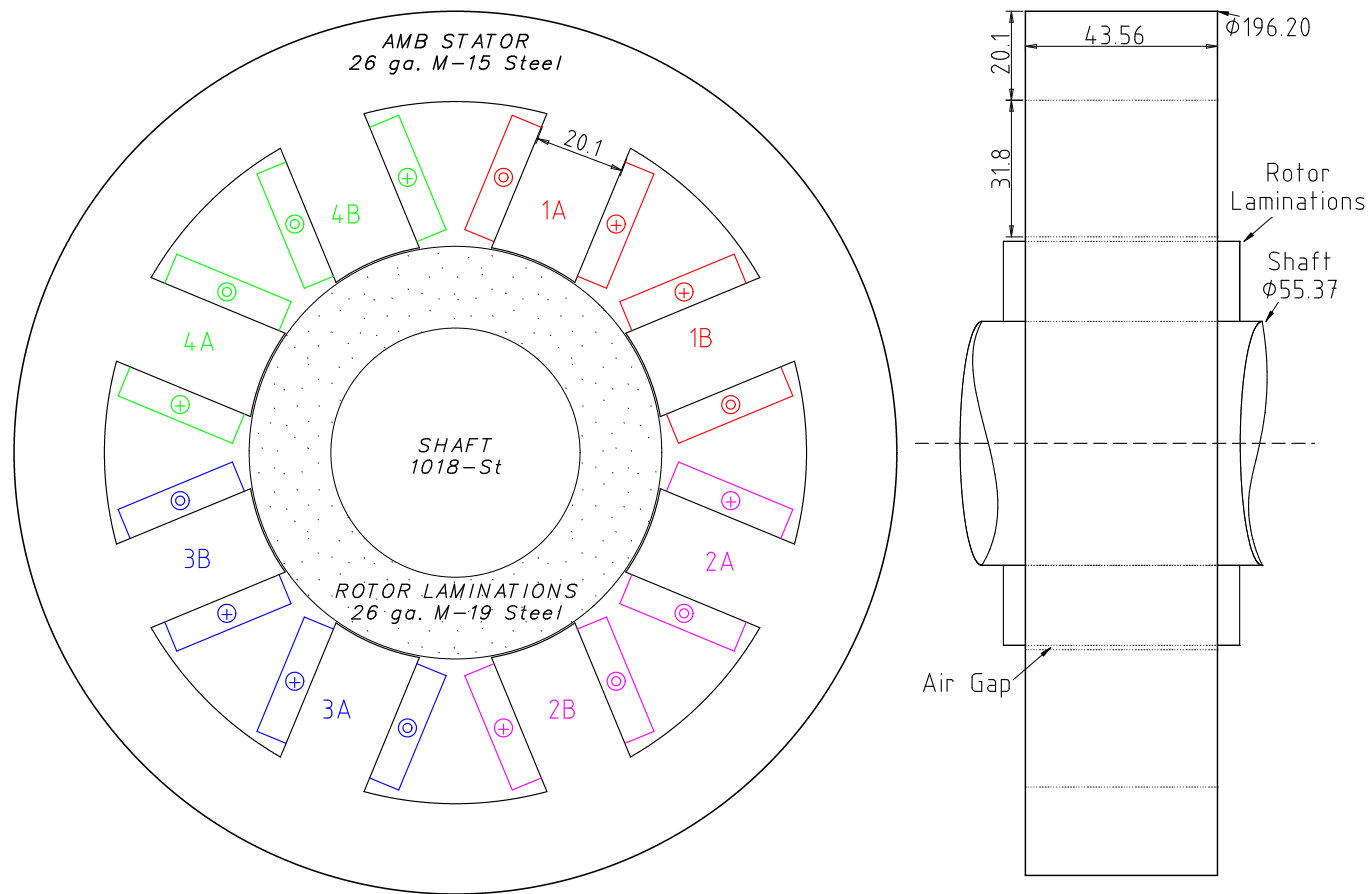

(b) Disturbance AMB.

Figure 4.16: Front and side views of the radial AMBs showing materials and dimensions (air gap is not drawn to scale). Control quadrants are labeled 1-4 and shown in different colors. 


\subsubsection{Modeling of Additional Components}

\subsubsection{Power Amplifiers}

The power amplifier dynamics are strongly influenced by the load impedance, i.e., resistance and inductance of AMB windings, in addition to back-EMF and eddy current effects due to translation and rotation of the shaft (Maslen et al., 2006). The first two effects can be modeled and verified with experimental measurements, whilst the third is more challenging to quantify. Through the use of laminated AMB rotor and stator components eddy current effects are significantly minimized and this can largely be ignored in radial AMBs. The internal circuit model of a power amplifier was available from the vendor. However, this model was based on several parameters which were manually adjusted with trim pots and are as a result difficult to obtain exactly. The small-signal actuator response was obtained experimentally using a system analyzer to supply a swept sinusoid to the amplifier while the rotor was centered in the air gap using plastic shims. The output current in the windings was measured using a current transducer. The following transconductance transfer function was fit to the experimental frequency response presented in Figure 4.17a,

$$
G_{\mathrm{as}}(s)=\frac{\mathrm{DC} \text { gain }}{\left(s+p_{\mathrm{a} 1}\right)\left(s+p_{\mathrm{a} 2}\right)\left(s^{2}+2 \xi_{\mathrm{a}} \omega_{\mathrm{a}} s+\omega_{\mathrm{a}}^{2}\right)} \mathrm{A} / \mathrm{V}
$$

where $p_{\mathrm{a} 1}=13820 \mathrm{rad} / \mathrm{s}, p_{\mathrm{a} 2}=28270 \mathrm{rad} / \mathrm{s}, \omega_{\mathrm{a}}=16210 \mathrm{rad} / \mathrm{s}$, and $\xi_{\mathrm{a}}=0.55$. The Copley 422 amplifiers for NDE and DE support AMBs were configured for a DC gain of $2.5 \mathrm{~A} / \mathrm{V}$, and internally compensated for a $-3 \mathrm{~dB}$ bandwidth of $2.5 \mathrm{kHz}$ and $10^{\circ}$ phase lag at $255 \mathrm{~Hz}$. Four instances of the transfer function $G_{\text {as }}(s)$ were combined into a $4 \times 4$ MIMO state space model $\left(A_{\mathrm{a}}, B_{\mathrm{a}}, C_{\mathrm{a}}\right)$ with a total of 16 states $x_{\mathrm{a}}=\left[\begin{array}{llll}x_{\mathrm{a} 1}, & x_{\mathrm{a} 2}, & \ldots, & x_{\mathrm{a} 16}\end{array}\right]^{\mathrm{T}}$. The controller output voltage vector $u$ serves as the input and the output is the perturbation current vector $i_{\mathrm{p}}$. Combining this description into the model (4.14) and (4.15) increases the number of 
states by twelve producing

$$
\begin{aligned}
{\left[\begin{array}{c}
\dot{x}_{\mathrm{m}} \\
\dot{x}_{\mathrm{a}}
\end{array}\right] } & =\left[\begin{array}{cc}
\hat{A}_{\mathrm{m}} & B_{1 \mathrm{~m}} K_{\mathrm{i}} C_{\mathrm{a}} \\
0 & A_{\mathrm{a}}
\end{array}\right]\left[\begin{array}{c}
x_{\mathrm{m}} \\
x_{\mathrm{a}}
\end{array}\right]+\left[\begin{array}{c}
0 \\
B_{\mathrm{a}}
\end{array}\right] u+\left[\begin{array}{c}
B_{2 \mathrm{~m}} \\
0
\end{array}\right] F_{\mathrm{w}} \\
y_{\mathrm{s}} & =C_{\mathrm{s}} x_{\mathrm{s}} .
\end{aligned}
$$

The use of different power amplifiers (Copley 413) and a higher load impedance resulted in the mid-span AMB actuator amplifier having a lower small-signal bandwidth than the support AMBs. Using a third-order transfer function template, the small-signal dynamics of the amplifier is

$$
G_{\mathrm{ad}}(s)=\frac{\text { DC gain }}{\left(s+p_{\mathrm{a} 1}\right)\left(s^{2}+2 \xi_{\mathrm{a}} \omega_{\mathrm{a}} s+\omega_{\mathrm{a}}^{2}\right)} \mathrm{A} / \mathrm{V},
$$

where $p_{\mathrm{a} 1}=10,460 \mathrm{rad} / \mathrm{s}, \omega_{\mathrm{a}}=9,425 \mathrm{rad} / \mathrm{s}$, and $\xi_{\mathrm{a}}=0.66$. The Copley 413 amplifiers for MID disturbance AMBs were configured for a DC gain of $2.5 \mathrm{~A} / \mathrm{V}$, and internally compensated for a $-3 \mathrm{~dB}$ bandwidth of $1.5 \mathrm{kHz}$ and $10^{\circ}$ phase lag at $155 \mathrm{~Hz}$. The normalized frequency response is shown in Figure 4.17b.

\subsubsection{Position Sensors and Anti-Aliasing Filters}

Four differential eddy current type displacement probes per AMB were used to measure shaft motion in the direction of the AMB control quadrants. The sensors were distanced 8 $\mathrm{mm}$ from the AMB lamination stacks to avoid noncolocation and their dynamics modeled by a constant gain of $8 \mathrm{mV} / \mu \mathrm{m}$. The analog signal conditioning circuitry included an eighth order inverse Chebychev low-pass anti-aliasing filter with a $4 \mathrm{kHz}$ stop band and $-30 \mathrm{~dB}$ minimum attenuation specification. While this filter has flat magnitude response up to $3 \mathrm{kHz}$, it contributes a $40^{\circ}$ phase lag at $1 \mathrm{kHz}$. The sensor and anti-aliasing filter was represented by a state space model with four-entry voltage output vector $y_{\mathrm{s}}$, and a state space model $\left(A_{\mathrm{s}}, B_{\mathrm{s}}, C_{\mathrm{s}}\right)$ containing a 32 element state vector $x_{\mathrm{s}}$. Combining this model 


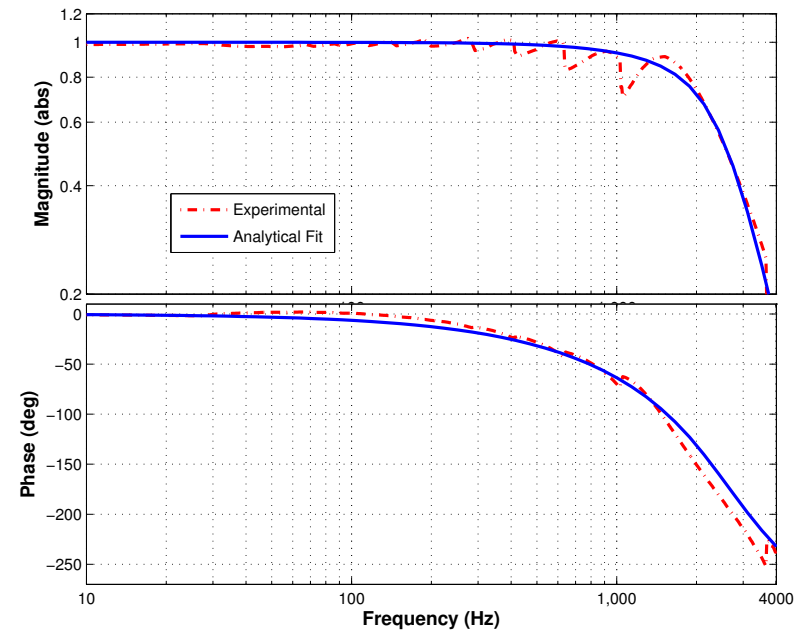

(a) Copley 422 amplifier and DE AMB.

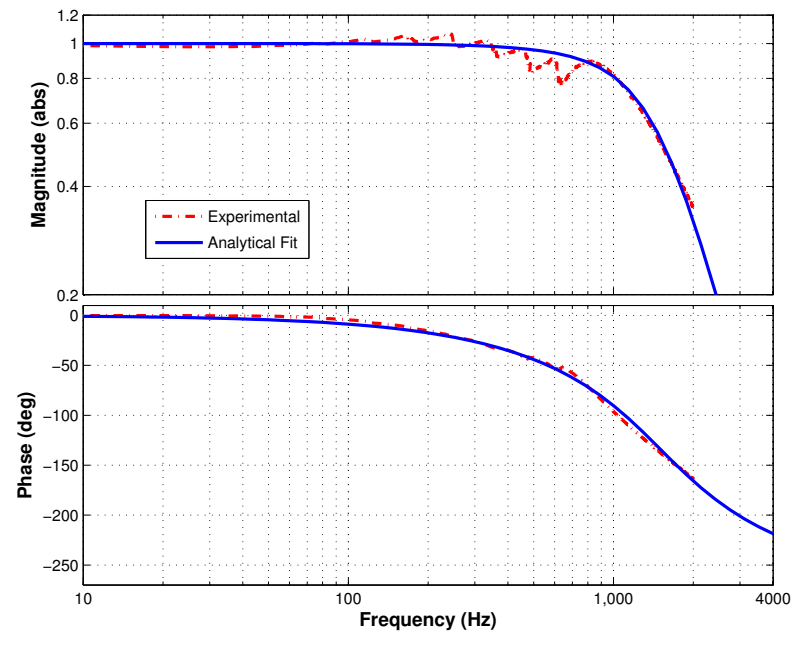

(b) Copley 413 amplifier and MID AMB.

Figure 4.17: Normalized Bode plots of power amplifier response from command voltage input to AMB coil current output. The dash-dotted line is from the experiment and the solid line is from the model.

with the linearized rotor-AMB models gives

$$
\begin{aligned}
{\left[\begin{array}{c}
\dot{x}_{\mathrm{m}} \\
\dot{x}_{\mathrm{s}} \\
\dot{x}_{\mathrm{a}}
\end{array}\right] } & =\left[\begin{array}{ccc}
\hat{A}_{\mathrm{m}} & 0 & B_{1 \mathrm{~m}} K_{\mathrm{i}} C_{\mathrm{a}} \\
B_{\mathrm{s}} C_{\mathrm{m}} & A_{\mathrm{s}} & 0 \\
0 & 0 & A_{\mathrm{a}}
\end{array}\right]\left[\begin{array}{c}
x_{\mathrm{m}} \\
x_{\mathrm{s}} \\
x_{\mathrm{a}}
\end{array}\right]+\left[\begin{array}{c}
0 \\
0 \\
B_{\mathrm{a}}
\end{array}\right] u+\left[\begin{array}{c}
B_{2 \mathrm{~m}} \\
0 \\
0
\end{array}\right] C_{\mathrm{s}} x_{\mathrm{s}} .
\end{aligned}
$$

\subsubsection{Digital Signal Chain}

Several additional filters are part of the signal chain that interfaces the digital controller with the rotor-AMB system. Any filter that contributes phase lag to the plant response within the controller bandwidth must be included in the plant model (Brown et al., 2005). The analog front-end of DSP board includes a fourth order $30 \mathrm{kHz}$ elliptical anti-aliasing filter on all the inputs and a single pole $50 \mathrm{kHz}$ output reconstruction filter on all the outputs. The magnitude response of these two filters is flat over the bandwidth considered for the MBTRI system, and their phase lags at $1 \mathrm{kHz}$ are less than $5^{\circ}$ (see Figure $4.19 \mathrm{a}$ ). The sampled 


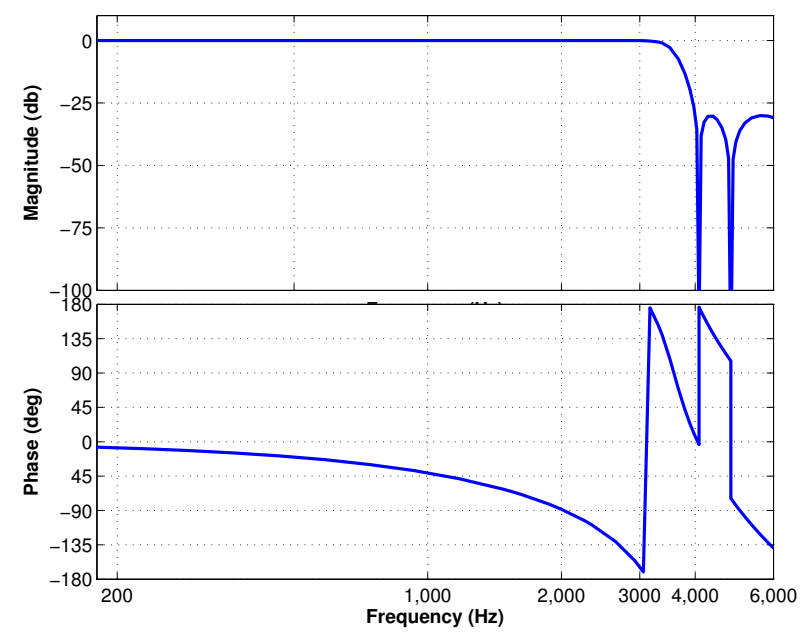

Figure 4.18: Bode plots of the frequency response of the displacement sensor an eighth order inverse Chebychev anti-aliasing filter.

nature of digital control systems causes outputs to be updated at discrete intervals equal to the sampling period, $T_{\mathrm{S}}=\frac{1}{12000 \mathrm{~Hz}}=83.3 \mu \mathrm{s}$. The delay between successive updates manifests itself as a phase lag in the frequency domain (Skogestad \& Postlethwaite, 2005). To model the contribution of this DSP computational delay to the system dynamics, a second order Padé approximation is used to obtain a proper rational transfer function for the delay

$$
e^{-T_{\mathrm{S}} s} \approx\left(1-0.25 T_{\mathrm{S}} s\right)^{2} /\left(1+0.25 T_{\mathrm{S}} s\right)^{2}
$$

The above computational delay contributes the largest phase lag of the electronic signal path, $23^{\circ}$ at $1 \mathrm{kHz}$. The combined frequency response of all the filters in the signal chain from the sensors to the controller output waveform are shown in Figure 4.19b.

\subsection{Reduced Order Model}

The above sampling filters and the Padé time delay approximation contribute 28 additional states and are combined into a state space model $\left(A_{\mathrm{f}}, B_{\mathrm{f}}, C_{\mathrm{f}}\right)$. This model is placed in series with Equations (4.20) and (4.21), to give a complete analytical model of the rotor-AMB 


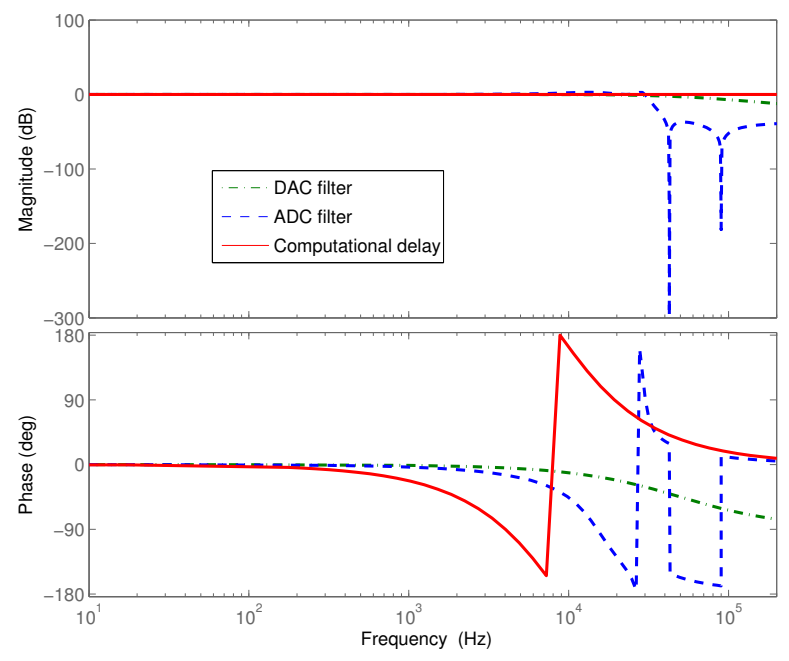

(a) Individual responses.

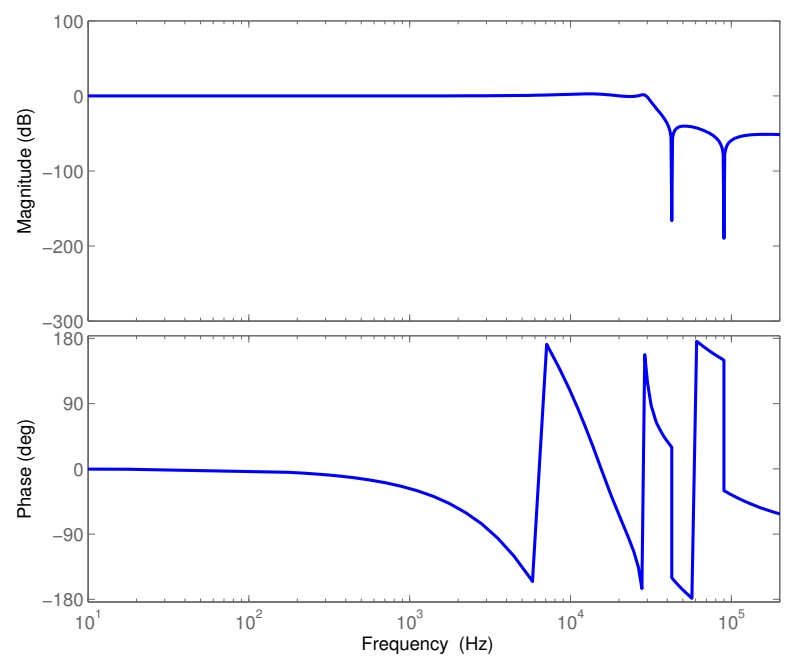

(b) Overall response of digital signal chain.

Figure 4.19: Frequency response of sampling filters and computational delay approximation.

system

$$
\begin{aligned}
{\left[\begin{array}{c}
\dot{x}_{\mathrm{m}} \\
\dot{x}_{\mathrm{s}} \\
\dot{x}_{\mathrm{a}} \\
\dot{x}_{\mathrm{f}}
\end{array}\right]=} & {\left[\begin{array}{cccc}
\hat{A}_{\mathrm{m}} & 0 & B_{1 \mathrm{~m}} K_{\mathrm{i}} C_{\mathrm{a}} & 0 \\
B_{\mathrm{s}} C_{\mathrm{m}} & A_{\mathrm{s}} & 0 & 0 \\
0 & 0 & A_{\mathrm{a}} & 0 \\
0 & B_{\mathrm{f}} C_{\mathrm{s}} & 0 & A_{\mathrm{f}}
\end{array}\right]\left[\begin{array}{c}
x_{\mathrm{m}} \\
x_{\mathrm{s}} \\
x_{\mathrm{a}} \\
x_{\mathrm{f}}
\end{array}\right] } \\
& +\left[\begin{array}{c}
0 \\
0 \\
B_{\mathrm{a}} \\
0
\end{array}\right] u+\left[\begin{array}{c}
B_{2 \mathrm{~m}} \\
0 \\
0 \\
0
\end{array}\right] F_{\mathrm{w}},
\end{aligned}
$$

The order of the overall model presented in Equation (4.23) is 92, which remains a relatively large number of states to retain for model-based control design. It is possible to further reduce the model order by combining the responses of all the electrical, electronic 
components into a single equivalent input-output model. The gain plus time delay model chosen for the electrical and electronic components has 16 states, $x_{d}$, and a state space description

$$
\begin{aligned}
\dot{x}_{d}(t) & =A_{\mathrm{d}} x_{\mathrm{d}}(t)+B_{\mathrm{d}} y_{\mathrm{r}}(t), \\
y(t) & =C_{\mathrm{d}} x_{\mathrm{d}}(t)+D_{\mathrm{d}} y_{\mathrm{r}}(t) .
\end{aligned}
$$

A fourth-order Padé approximation is used for each of the four channels and the delay time is tuned to match the overall phase response. The output of the rotor-AMB model is the displacement at the four sensing planes which forms the input to the the gain plus time delay model. The time delay models for each control channel are independent fourth-order Padé approximations that match the phase lag of the components in the signal path, i.e., power amplifiers, sensor signal conditioning filters, and DSP sampling and computational delay. The DC gain of each channel is set to be the product of individual gains of the components in the signal path. By inserting the delay model in series with the rotor-AMB model we arrive at the following compact representation of the system requiring only 36 states,

$$
G(s):=\left[\begin{array}{c}
\dot{x}_{\mathrm{r}}(t) \\
\dot{x}_{\mathrm{d}}(t) \\
y(t)
\end{array}\right]=\left[\begin{array}{cc}
\hat{A}_{\mathrm{r}} & 0 \\
B_{\mathrm{d}} C_{\mathrm{r}, \mathrm{s}} & A_{\mathrm{d}} \\
D_{\mathrm{d}} C_{\mathrm{r}, \mathrm{s}} & C_{\mathrm{d}}
\end{array}\right]\left[\begin{array}{c}
x_{\mathrm{r}}(t) \\
x_{\mathrm{d}}(t)
\end{array}\right]+\left[\begin{array}{c}
\hat{B}_{\mathrm{r}} \\
0 \\
0
\end{array}\right] u(t)
$$

The above approach allows for a lower order model with improved numerical conditioning than would have been achieved by considering the amplifier, sensor, AA filter, sampling and delay dynamics individually. The nominal plant represented by the state space model in Equation (4.27) has four rotor displacement outputs and four amplifier voltage inputs. For subsequent analysis and control design the inputs and outputs are similarly ordered 
NDE-X, DE-X, NDE-Y, and DE-Y. At zero RPM the $x-y$ dynamics are uncoupled and Bode plots of the two-input, two-output subsystem represented by selecting only the dynamics in the $x$-direction from Equation (4.27) are shown in Figure 4.20. An axisymmetric rotor design ensures that the rotordynamics the $x$ and $y$ directions are identical, with the only slight differences introduced due to variations in the sensor and amplifier gains. The two prominent peaks in all four Bode plots at $224 \mathrm{~Hz}$ and $540 \mathrm{~Hz}$, correspond to the first and second bending modes $\mathrm{Nc} 3$ and Nc4, respectively. The peak at $982 \mathrm{~Hz}$ corresponds to the third bending mode Nc5. The small response at Nc5 is expected from the free-free mode shape analysis (Figure 4.8). The off-diagonal Bode plots (b) and (c) represent the response from the DE-X amplifier input, to the NDE-X sensor, and NDE-X amplifier input to the DE-X sensor. Notably, the DC gain is lower than the diagonal channels, and there is a notch a low frequency corresponding to a LHP zero for Nc3. The singular value plot of the full four-input, four-output nominal plant model is shown in Figure 4.21.

\subsection{Validation using System Identification}

Before the model developed in the preceding sections may be used for control design, it is desirable to confirm its validity using system identification techniques. Though we are confident about the structure of the model, i.e., from the development of rotordynamic and magnetostatic models from first principles, there is a need to confirm the overall inputoutput response and the frequencies of the bending modes. Extensive discussion and comparison of identification methods used for rotor-AMB systems is available in the literature (Gahler et al., 1997; Losch, 2002). A comprehensive treatise on the theory and practice of system identification is recommended for a general background (Ljung, 1998).

As rotor-AMB systems are open-loop unstable, closed-loop system identification is required whereby a feedback controller (preferably with as low stiffness as possible) is used during the identification procedure. A straightforward decentralized PID-like com- 


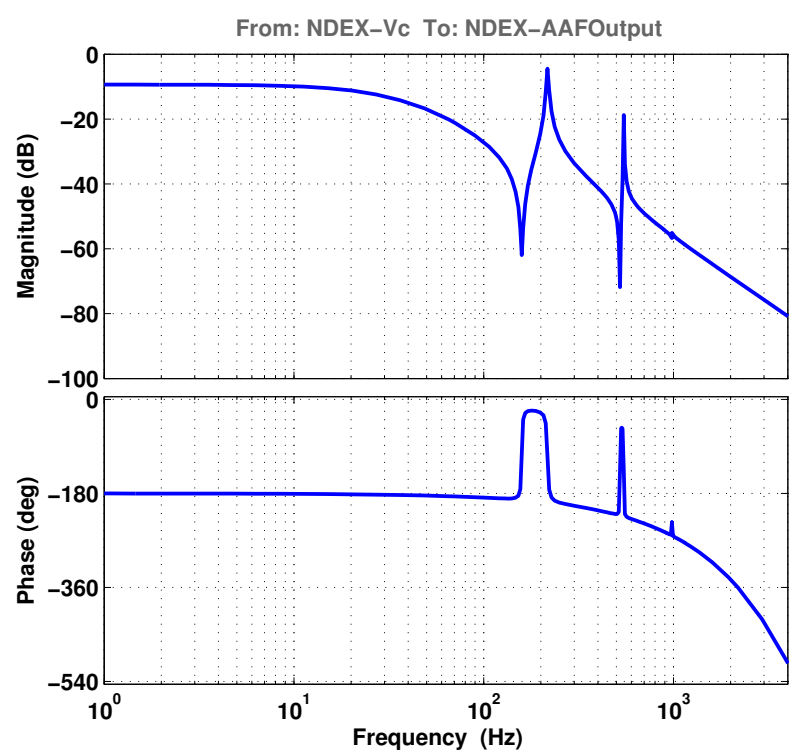

(a)

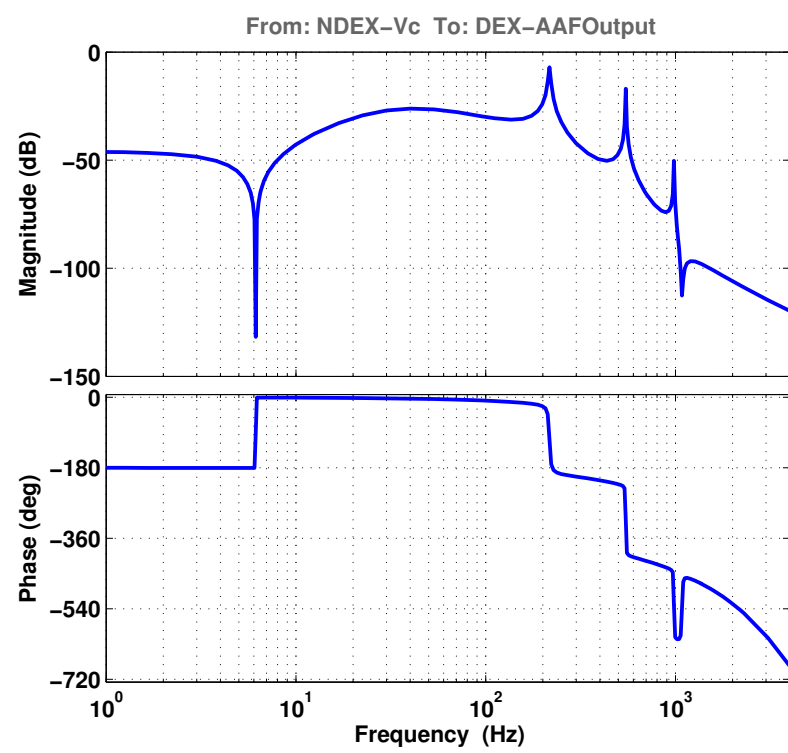

(c)

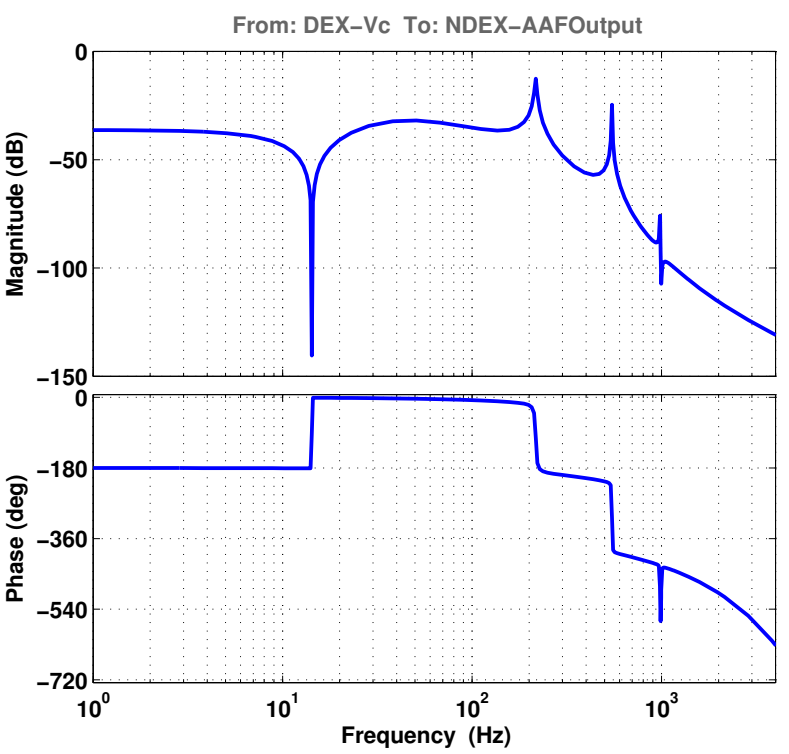

(b)

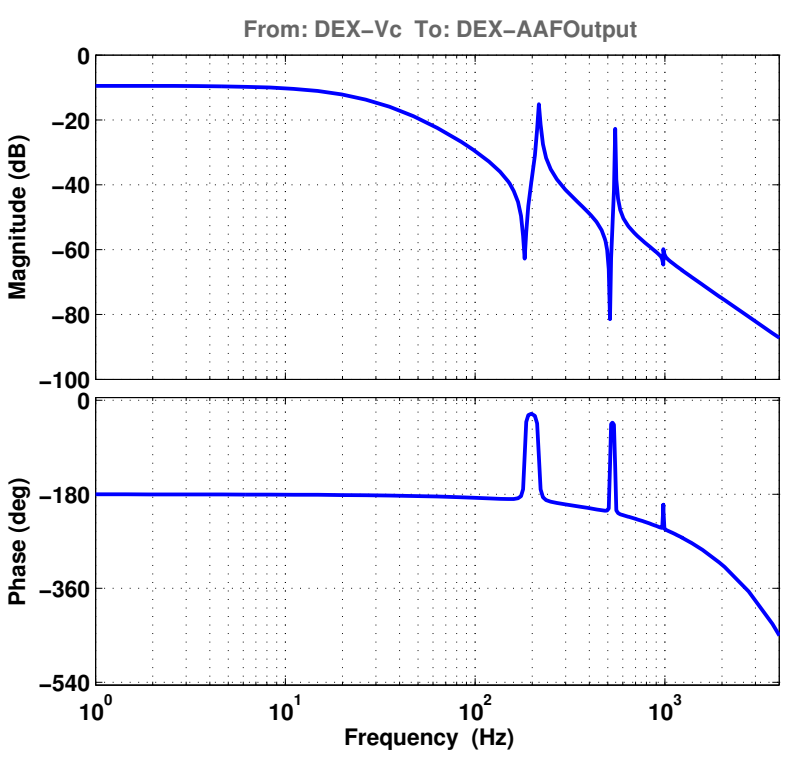

(d)

Figure 4.20: Bode plots of plant dynamics in the $x$-axis only. 


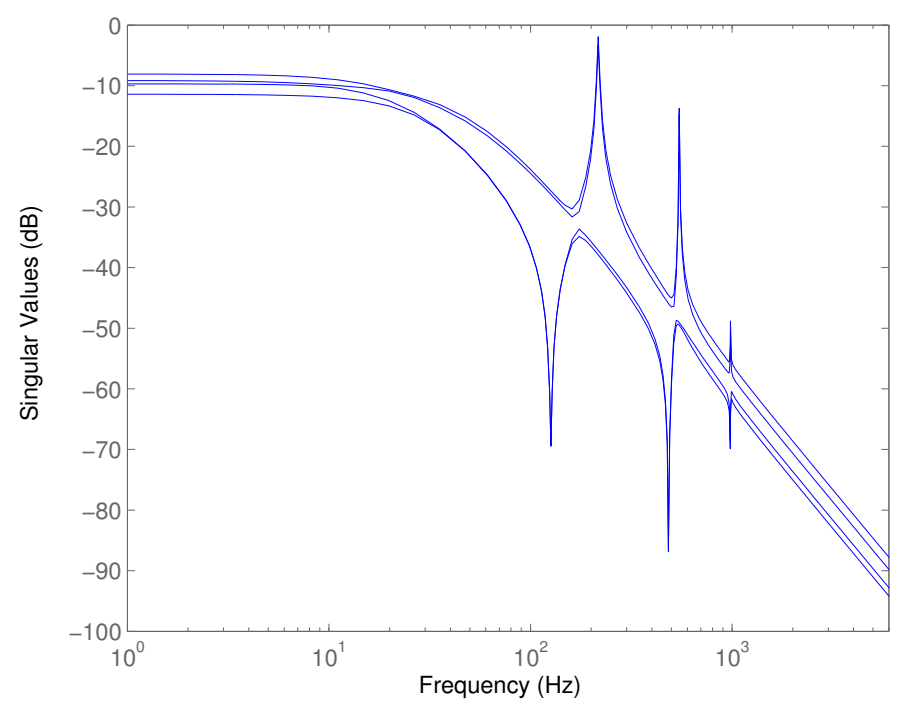

Figure 4.21: Singular value plot of nominal plant model.

pensation algorithm was adopted (Mushi et al., 2010). The kernel of the algorithm was a "velocity" type PID implementation with a second-order filtered derivative. The discrete time form of the algorithm

$$
u(k)=u(k-1)+K_{\mathrm{s}} K_{\mathrm{p}}\left[(e(k)-e(k-1))+\frac{T_{s}}{T_{\mathrm{i}}} e(k)+\frac{T_{\mathrm{d}}}{T_{S}}(e(k)-2 e(k-1)-e(k-2))\right]
$$

where the index $k$ reflects a single sample, error signal is $e(k), K_{\mathrm{s}}$ is the sensor gain, $K_{\mathrm{p}}$ is the proportional gain, $T_{\mathrm{i}}$ is the integrator time constant, $T_{\mathrm{d}}$ is the derivative time, and $T_{S}=1 / 12000$ is the sample time of the digital controller. As the PID algorithm alone cannot completely stabilize the system, added in series are a phase lead filter, low pass filters and several notch filters to provide compensation for the bending modes. The notches are typically placed approximately $10 \mathrm{~Hz}$ below the mode they are intended to attenuate. This takes advantage of the increase in phase for a few $\mathrm{Hz}$ after the notch frequency to provide robust damping (phase stabilization), as opposed to magnitude stabilization if the notch and the target mode were coincident in frequency. The notches are designed and implemented 
in discrete time as second-order infinite impulse response filters of the form

$$
G_{\mathrm{notch}}(z)=\frac{1+\alpha}{2}\left(\frac{1-2 \beta z^{-1}+z^{-2}}{1-\beta(1+\alpha) z^{-1}+\alpha z^{-1}}\right),
$$

where $0<\alpha<1$ and $-1 \leq-\beta=\cos \left(f_{n} T_{s}\right) \leq 1$. The width of the notch is determined by $\alpha$ while $f_{n}$ represents the notch frequency in rad/s. The phase lead ("bump") filter is crucial for the damping of the first bending mode $\mathrm{Nc} 3$ and has the continuous time representation

$$
G_{\mathrm{pbf}}(s)=\frac{1}{1-\varepsilon}\left(1-\frac{\varepsilon}{(\tau s)^{2}+2 \zeta \tau s+1}\right),
$$

where $\varepsilon$ sets the amount of phase lead, $\zeta$ determines the Q-factor, and $\tau$ controls the center frequency to achieve a maximum of $48^{\circ}$ of phase lead at $829 \mathrm{~Hz}$ with a penalty of $11 \mathrm{~dB}$ of added gain at high frequency. This particular filter is more efficient than the classical lead compensator which would have a larger high frequency gain for the same amount of phase lead. Figure 4.22 illustrates the frequency response of the phase lead filter described above. See Table 4.7 for a list of the parameters used. Bode plots of the magnitude and phase characteristics of NDE-X and DE-X channels are provided in Fig. 4.23.

Table 4.7: PID controller parameters for online system identification.

\begin{tabular}{|c|c|c|c|c|}
\hline & DE-X & DE-Y & NDE-X & NDE-Y \\
\hline \hline Sensor gain, $K_{\mathrm{S}}$ [mil/V] & 38.57 & 35.73 & 35.43 & 38.26 \\
\hline Proportional gain, $K_{\mathrm{p}}$ & \multicolumn{4}{|c|}{0.25} \\
\hline Integration time constant, $T_{\mathrm{i}}$ & \multicolumn{4}{|c|}{$0.01 \mathrm{~s}$} \\
\hline Derivative time constant, $T_{\mathrm{d}}$ & \multicolumn{4}{|c}{$0.003 \mathrm{~s}$} \\
\hline Notch 1 frequency & $534 \mathrm{~Hz}$ & $536 \mathrm{~Hz}$ & $536 \mathrm{~Hz}$ & $536 \mathrm{~Hz}$ \\
\hline Notch 2 frequency & $970 \mathrm{~Hz}$ & $972 \mathrm{~Hz}$ & $970 \mathrm{~Hz}$ & $970 \mathrm{~Hz}$ \\
\hline Notch 3 frequency & $1250 \mathrm{~Hz}$ & $1250 \mathrm{~Hz}$ & $1200 \mathrm{~Hz}$ & $1200 \mathrm{~Hz}$ \\
\hline First order low pass filter & \multicolumn{3}{|c|}{ First order Butterworth, $\omega_{\mathrm{c}}=600 \mathrm{~Hz}$} \\
\hline Phase lead filter & \multicolumn{5}{|c}{$\varepsilon=0.65 ; \tau=0.00012 ; \zeta=0.5$} \\
\hline Moving average filter & \multicolumn{5}{|c|}{$5 \mathrm{tap}$} \\
\hline
\end{tabular}




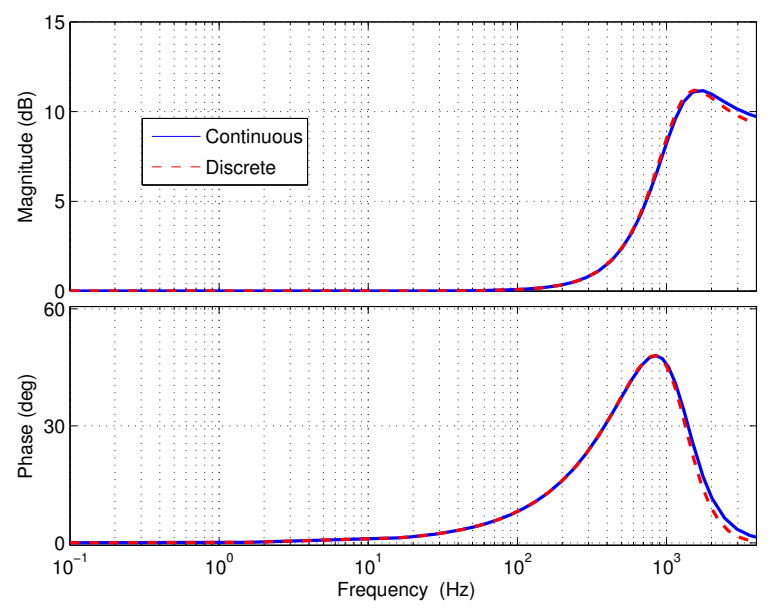

Figure 4.22: Bode plot of phase lead filter used in PID controller.

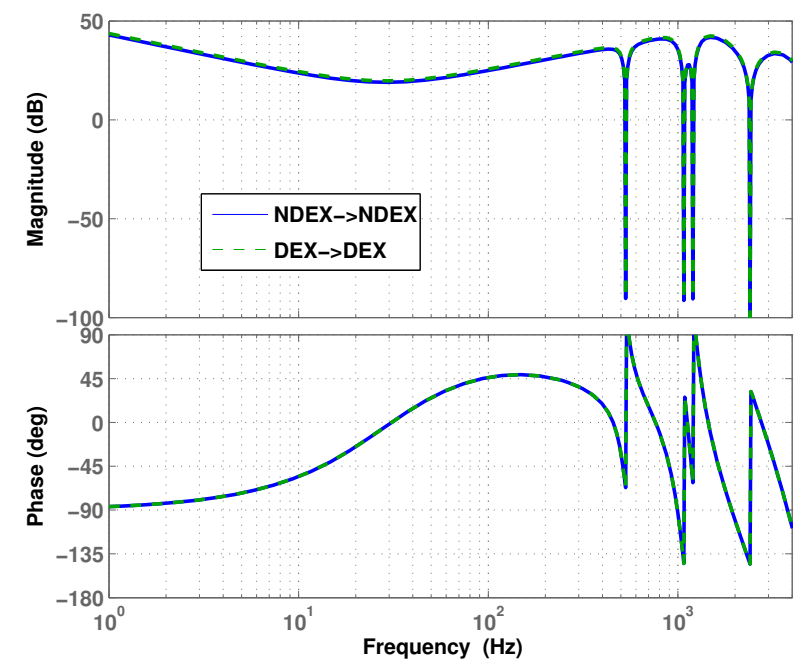

Figure 4.23: Bode plot of PID controller for NDE-X and DE-X channels.

Once the rotor has been suspended by a suitable controller, a system analyzer was used to superpose a fixed amplitude swept-sinusoid from $10 \mathrm{~Hz}$ to $1200 \mathrm{kHz}$ onto the controller output signal. The response from the desired sensor AAF filter was recorded. The stimulus and responses were measured one at a time for all four inputs and outputs, provided a total of sixteen frequency response functions. Key parameters of the model in the previous 
section were modified to reconcile differences observed. Figure 4.24 shows Bode plots of the input-output response along one of the four channels of the model and compares this to experimental transfer function measurements from the test rig. The close agreement of the magnitude and phase response over the range from $1 \mathrm{~Hz}$ to $1 \mathrm{kHz}$ confirmed the accuracy of the above model.

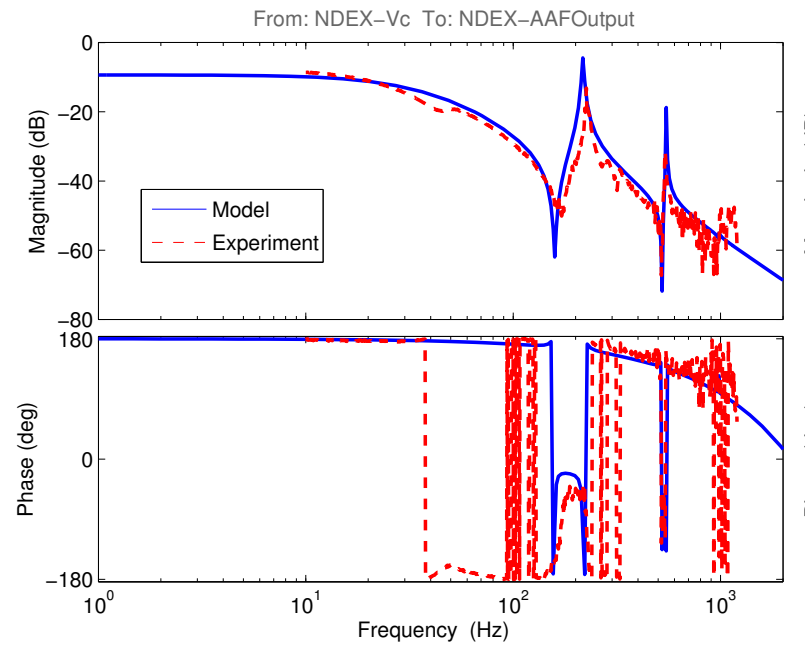

(a)

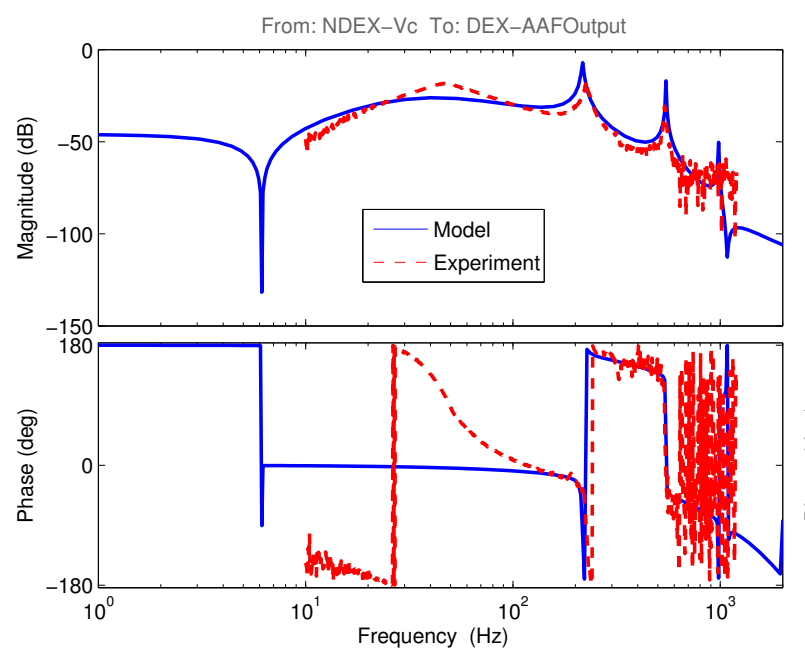

(c)

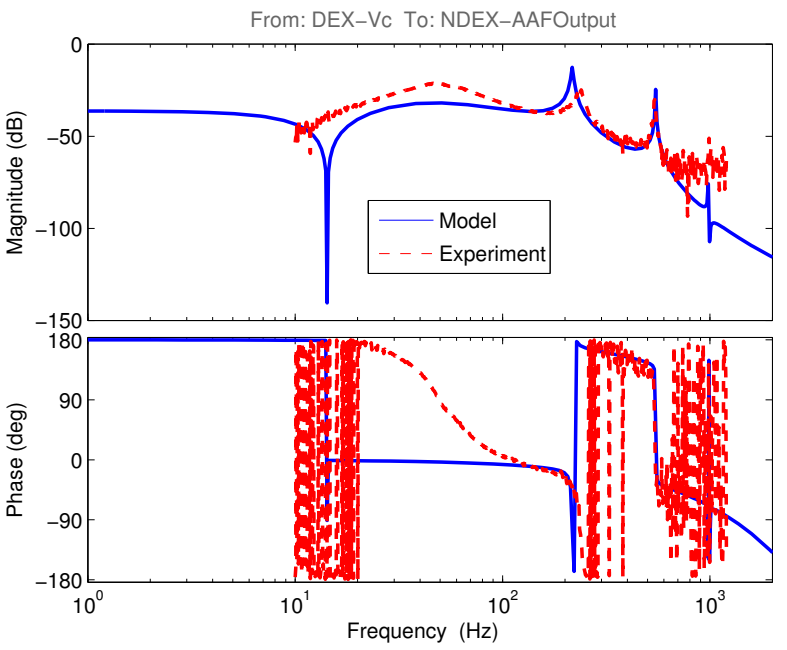

(b)

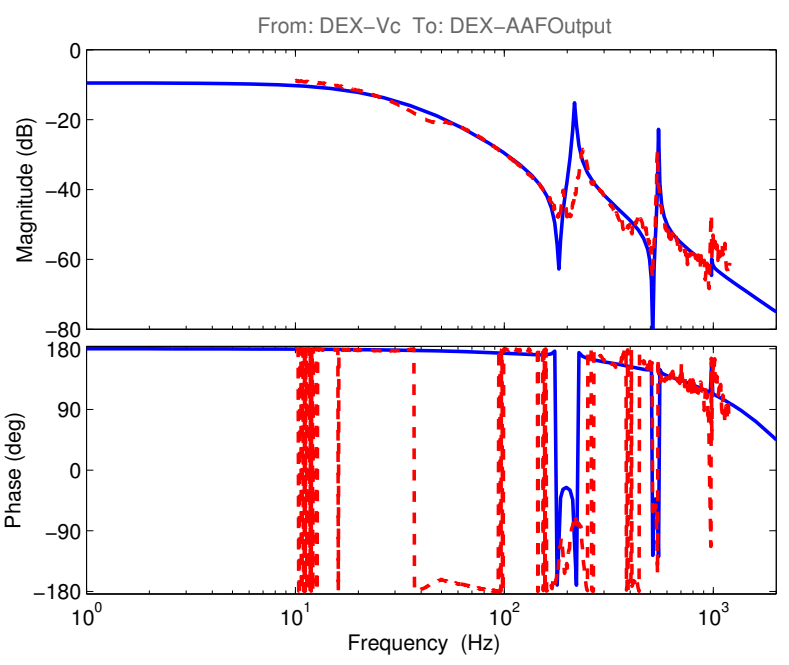

(d)

Figure 4.24: Bode plots comparing experimental frequency response to system model along the $x$ axis. The response is measured from power amplifier voltage input to sensor signal conditioning circuit output. 


\subsection{Bandwidth Analysis}

\subsubsection{Available Bandwidth}

According to Stein (2003), the knowledge of the available bandwidth $\Omega_{a}$ of the components in the feedback path is critical to evaluating the limits of achievable performance. The nominal rotor-AMB system has a pair of RHP poles at $191 \mathrm{rad} / \mathrm{s}(30.4 \mathrm{~Hz})$ and $318 \mathrm{rad} / \mathrm{s}$ $(50.8 \mathrm{~Hz})$ corresponding to the unstable rigid body rotor modes. To this end the bandwidths, in the 3-dB gain sense, of various components are considered:

- DSP: the sample rate is $12 \mathrm{kHz}$, allowing a control algorithm to respond to signals up to the Nyquist sampling limit of $6 \mathrm{kHz}$.

- Sensors and AAFs: displacement sensors have a flat response beyond $10 \mathrm{kHz}$, however, the AAF is the limiting components, with a bandwidth of $3.5 \mathrm{kHz}$.

- Actuator: The response of the power amplifier and AMB are combined. The smallsignal response of the pair is $2.5 \mathrm{kHz}$ for the support AMBs.

- Rotordynamics: The first three free-free bending modes of the rotor are at $224 \mathrm{~Hz}$, $540 \mathrm{~Hz}$ and $982 \mathrm{~Hz}$. Higher bending modes can be ignored so long as controller gain roll-off is enforced at high frequencies. Since we have endeavored to accurately model the impact of flexible modes on the dynamics, so long as they don't change significantly, they are not considered to have limiting effect on the achievable bandwidth.

From the above listing, we can state an achievable bandwidth $\Omega_{\mathrm{a}}$ of approximately $2 \mathrm{kHz}$. The estimated minimum peak sensitivity $s_{\min }$ for given a trapezoid sensitivity function template (see Figure 3.9) may be calculated using Equation (3.20). Using information about the two unstable poles, and assuming that the peak sensitivity occurs in the vicinity 
of the second rigid body mode, i.e., $\Omega_{\mathrm{m}}=320 \mathrm{rad} / \mathrm{s}$, therefore,

$$
\begin{aligned}
s_{\min } & \approx \exp \left(\frac{320+\pi(318+191)}{2200}\right) \\
& =2.39
\end{aligned}
$$

which corresponds to gain margin of at least $4.7 \mathrm{~dB}$ and a phase margin of at least $24^{\circ}$. These approximate calculations are encouraging and indicate that given the current hardware, stabilizing controllers can be built to meet the performance specifications. A measured output sensitivity function of the rotor-AMB system supported by the PID controller (see Figure 4.25) designed during system identification reveals the predicted $s_{\min }$ and assumptions made about the shape of the sensitivity function are relatively accurate.

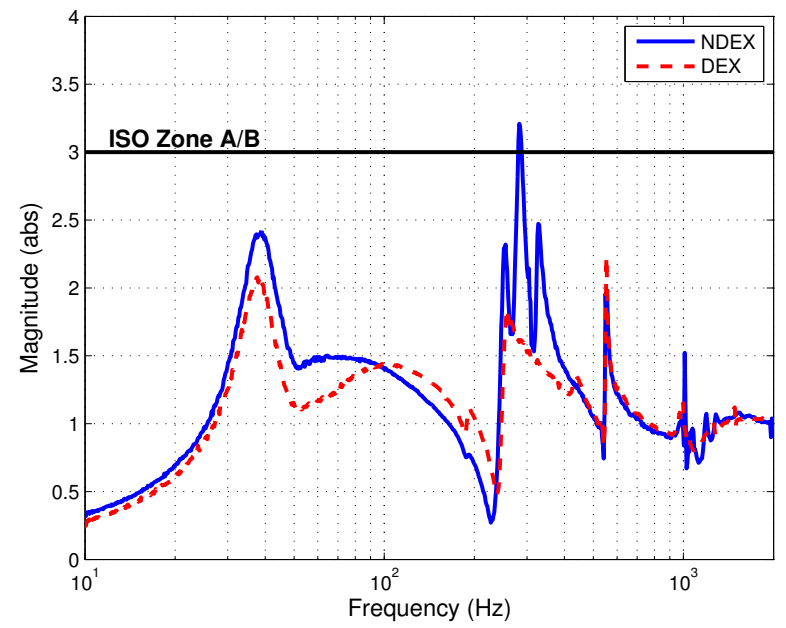

Figure 4.25: Output sensitivity of NDEX and DEX channels of collocated PID controller.

\subsubsection{Generating Cross-Coupled Stiffness}

Generating mechanical stiffness using an electromagnetic actuator requires real-time feedback of the rotor position for the controller to calculate the appropriate current and command the amplifiers (Ulbrich, 1988; Cloud, 2007). The CCS force produced by the midspan 
AMB is a linear function of the rotor position and is expressed as

$$
\begin{aligned}
F_{\mathrm{xc}} & =Q\left[\begin{array}{cc}
0 & 1 \\
-1 & 0
\end{array}\right]\left[\begin{array}{l}
x \\
y
\end{array}\right], \\
& =\left[\begin{array}{cc}
K_{\mathrm{i}, \text { mid }} & 0 \\
0 & K_{\mathrm{i}, \text { mid }}
\end{array}\right]\left[\begin{array}{c}
i_{\mathrm{p}, \mathrm{x}} \\
i_{\mathrm{p}, \mathrm{y}}
\end{array}\right]+\left[\begin{array}{cc}
K_{\mathrm{x}, \text { mid }} & 0 \\
0 & K_{\mathrm{x}, \text { mid }}
\end{array}\right]\left[\begin{array}{l}
x \\
y
\end{array}\right],
\end{aligned}
$$

where $Q$ is the level of CCS to be generated, $x$ and $y$ are the rotor displacements measured by the sensors, $i_{\mathrm{p}, \mathrm{x}}$ and $i_{\mathrm{p}, \mathrm{y}}$ are the perturbation currents supplied to the windings, and $K_{\mathrm{x}, \mathrm{mid}}$ and $K_{\mathrm{i}, \mathrm{mid}}$ are the negative stiffness and current gain of the midspan AMB. With Equation (4.32) two assumptions are made: the displacement sensor and actuator are sufficiently colocated, and the actuator dynamics along the $x$ and $y$ axes are not coupled. The first assumption is justified ass the sensor is directly adjacent to the side of the AMB. Finite element analysis predicted coupling between the axes to be on the order of $10 \%$ of the magnitude of the diagonal terms. For simplicity, these off diagonal terms are ignored. Selecting the current as the subject of the equation results in

$$
\left[\begin{array}{c}
i_{\mathrm{p}, \mathrm{x}} \\
i_{\mathrm{p}, \mathrm{y}}
\end{array}\right]=\left[\begin{array}{cc}
-\frac{K_{\mathrm{x}}}{K_{\mathrm{i}}} & \frac{Q}{K_{\mathrm{i}}} \\
\frac{-Q}{K_{\mathrm{i}}} & -\frac{K_{\mathrm{x}}}{K_{\mathrm{i}}}
\end{array}\right]\left[\begin{array}{l}
x \\
y
\end{array}\right],
$$

which ignores the dynamics of digital sampling, the small-signal bandwidths of the power amplifiers, sensors and anti-aliasing filters (AAF). A more complete model for the production of the CCS accounting for the frequency dependence of the above components is

$$
\begin{aligned}
{\left[\begin{array}{c}
v_{\mathrm{c}, \mathrm{x}}(s) \\
v_{\mathrm{c}, \mathrm{y}}(s)
\end{array}\right] } & =\left[\begin{array}{cc}
-\frac{K_{\mathrm{x}}}{K_{\mathrm{i}}} & \frac{Q}{K_{\mathrm{i}}} \\
\frac{-Q}{K_{\mathrm{i}}} & -\frac{K_{\mathrm{x}}}{K_{\mathrm{i}}}
\end{array}\right]\left[\begin{array}{cc}
\frac{1}{G_{\mathrm{ad}} G_{\mathrm{s}} G_{\mathrm{DSP}}(s)} & 0 \\
0 & \frac{G_{\mathrm{s}}(s)}{G_{\mathrm{ad}} G_{\mathrm{s}} G_{\mathrm{DSP}}(s)}
\end{array}\right]\left[\begin{array}{c}
x(s) \\
y(s)
\end{array}\right], \\
& =K_{\mathrm{q}} G_{\mathrm{xc}}(s),
\end{aligned}
$$


where $v_{\mathrm{c}}(s)$ is the voltage command to the power amplifier to generate the desired perturbation current, $G_{\mathrm{s}}(s)$ is the sensor and $\mathrm{AAF}$ response, $G_{\mathrm{ad}}(s)$ is the small-signal response of the Copley 413 amplifier and one quadrant of the MID AMB (see Figure 4.17b), and $G_{\mathrm{DSP}}(s)$ is the time delay as a result of sampling and computation. The overall gain is $K_{\mathrm{q}}$ and $G_{\mathrm{xc}}(s)$ is the normalized dynamic response of $G_{\mathrm{ad}}(s), G_{\mathrm{s}}(s)$ and $G_{\mathrm{DSP}}(s)$. Our aim to generate $\mathrm{CCS}$ over a bandwidth exceeding the first rigid body rotor mode $\mathrm{Nc} 1$ with a phase lag from the components in the feedback path $<10^{\circ}$. Therefore, we can safely ignore the dynamics in $G_{\mathrm{xc}}(s)$. Excessive phase lag leads to the generation of direct stiffness instead of cross-coupled stiffness, i.e., non-zero diagonal terms of LHS of Equation (4.32) (Cloud, 2007). Figure 4.26 shows that the amplifier bandwidth is the critical factor if the phase lag is to be further minimized, followed by the sensor AAF. The amplifier slew rate and small signal bandwidth may be increased by operating power amplifiers from a higher voltage DC bus. When Cloud encountered amplifier phase lag limitations during experiments with CCS generation, a decision was made to replace the analog PWM amplifiers operating from 48V DC bus with digital PWM amplifiers operating from a $170 \mathrm{~V}$ bus. The latter demonstrated a higher small signal bandwidth and increased slew rate limit.

For a bias current level of $1 \mathrm{~A}, K_{\mathrm{i}}=182 \mathrm{~N} / \mathrm{A}$ and $K_{\mathrm{x}}=-654 \mathrm{~N} / \mathrm{mm}$, so $K_{\mathrm{q}}$ can be defined as

$$
K_{\mathrm{q}}(Q):=\left[\begin{array}{cc}
3590 & \frac{Q}{182} \\
-\frac{Q}{182} & 3590
\end{array}\right]
$$

The bias current is kept low to ensure that any direct stiffness produced by the midspan AMB does not cause produce rotor deflection that the support bearings and control algorithm cannot compensate for. The goal for the midspan AMB is to provide up to $4 \mathrm{MN} / \mathrm{m}$ of destabilizing CCS to encourage whirl at the natural frequency $f_{1} \approx 48 \mathrm{~Hz}$ of the first rotor mode. The estimated actuator force slew rate to produce this may be estimated by the following:

1. Choosing the worst case midspan rotor peak-to-peak vibration, $q_{\mathrm{w}}=50 \mu \mathrm{m}$. 


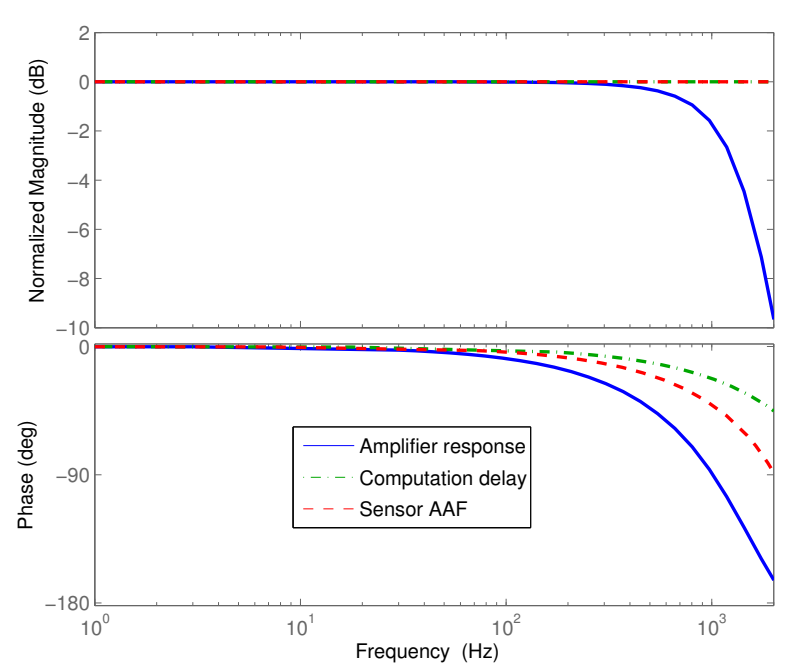

(a) Contribution of individual components to the response of the CCS feedback signal path.

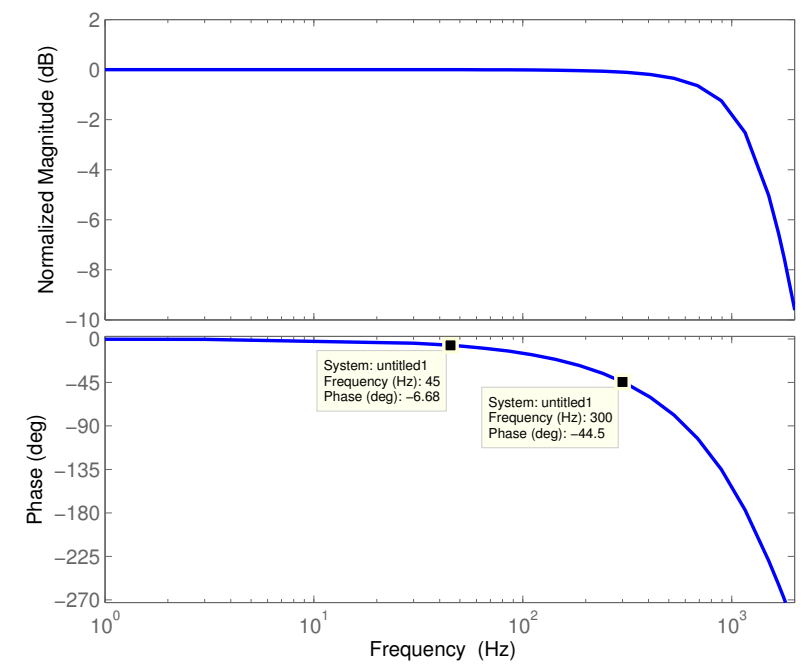

(b) Overall CCS dynamic response $G_{\mathrm{xc}}(s)$.

Figure 4.26: Dynamic response of the feedback loop necessary to generate CCS.

2. Multiplying $q_{\mathrm{w}}$ by maximum desired CCS to give the maximum force delivered $F_{\max }=$ $200 N$.

3. Multiply $F_{\max }$ by $1.5 f_{1}$ to give a slew rate of $14,400 \mathrm{~N} / \mathrm{s}$.

The estimated force slew rate of the mid-span actuator was predicted from the magnetic circuit model to exceed 50,000 N/s. Therefore, we expect not to have any issues producing the required CCS forces.

\subsection{Summary}

This chapter described the motivation and criteria for the design of the magnetic bearing test rig for rotordynamic instability (MBTRI). Using finite element rotordynamic analysis software a detailed rotor model was produced from the engineering drawings. The magnetic bearings were modeled with a combination of linear magnetic circuit analysis and finite element magnetostatics. The effects of power amplifier and signal conditioning circuit dynamics were also modeled and included in an overall state-space description of the 
rotor-AMB plant. The mechanism for generating the CCS force using the midspan AMB was discussed, and its was shown that sufficient bandwidth exists for this. Model reduction techniques were used to reduce the model order and simultaneously improve the numerical conditioning of the model. Differences between the dynamics of this compact analytical model were reconciled with experimental data from a closed-loop system identification procedure. This validated low-order plant model was now suitable for model-based control design. 


\section{Chapter 5}

\section{Robust Control Design and Analysis}

"Be a pessimist in analysis, then you can afford to be an optimist in design" -Ackermann (1993)

$\mu$-Synthesis is the only multivariable control design approach that directly addresses the robust performance problem, i.e., the design of a stabilizing control law that guarantees a performance specification for all plant model perturbations within a defined set (Honeywell \& Lockheed-Martin, 1996). The theory was introduced in the 1980s, and steadily improving commercial software tools have been available in the last decade. A few notable prototype industrial rotor-AMB systems utilizing $\mu$-synthesis have been documented in open literature (Losch et al., 1998; Fittro \& Knospe, 2002), but there is scant evidence the application of $\mu$-analysis techniques. These facts highlight the significant challenges which remain to be addressed in order for the full potential of these modern techniques to be realized. The most prominent challenge is the relatively high design complexity involved with these techniques and concerns regarding practical implementation and field troubleshooting. The last issue, field troubleshooting, is helped by the advent of remote diagnostic and communications capabilities of current AMB control systems that allow online system identification and auto-tuning (Losch, 2002; Swann, 2009). The second concern is less of an issue nowadays as the available computing power for executing real-time control algo- 
rithms continues to increase. The first issue mentioned looms large as the translation of engineering performance specifications into formal constraints in the form of weighting functions, uncertainty models, and other design criteria remain a largely heuristic process (Franchek, 1996). These issues are not unique to $\mu$-techniques, but symptomatic of the limited penetration of advanced multivariable control into industrial systems. One may argue why bother with an investment in $\mu$-synthesis if a hand-tuned compensator designed by a control practitioner has the potential to deliver similar performance (this is particularly relevant in the industrial control system community dominated by hand tuned PID-like compensators). Maslen \& Sawicki (2007) answer this question by suggesting that investments in $\mu$-techniques result in an improvement in the engineering process. The complexity and performance demanded of modern precision control systems is such that the notion when provided with a model and performance specification one can "turn a crank" and deliver a control law is naive (Garg, 2008). Assuming an accurate nominal plant model is available (a nontrivial task in many cases (Ogunnaike, 1996)), the remaining challenge is the development and continuous refinement of performance specifications and uncertainty models that yield controllers approaching the theoretical maximum achievable performance.

\subsection{Objectives}

The maintenance of a satisfactory steady state performance in response to residual rotor imbalance, electromagnetic disturbances affecting the measurement signals, and low frequency destabilizing forces at seal and impeller locations is the primary objective of AMB controllers in centrifugal compressor applications (Swanson et al., 2008). A satisfactory performance level in terms of allowable rotor displacement is quantified by existing turbomachinery industry standards (ISO 14839-2, 2004; API 617, 2002). In the process of meeting the vibration criteria, the controller must also ensure that the actuators are not driven to their slew rate or magnetic force saturation limits as this violates the linearity 
assumptions. Unless the controller has been designed to handle nonlinear behavior, no guarantees about the AMB performance can be made if these limits are exceeded. The stability and robustness of the closed-loop system to plant variations can be quantified by the damping ratio (or logarithmic decrement) of rotor modes and output sensitivity of the rotorAMB system (ISO 14839-3, 2006). The damping ratio of the rotor modes and the output sensitivity of the closed-loop system are measurable indicators of stability and robustness. For the MBTRI the requirements of the control system are formalized:

1. Stabilize all rotor modes within the controller bandwidth,

2. During run-up from 0 to $18,000 \mathrm{rpm}$ :

(a) Maintain rotor peak-peak vibration amplitudes within $30 \%$ of the auxiliary bearing clearance, i.e., within $75 \mu \mathrm{m}$.

(b) Maintain amplifier output current within $80 \%$ of the current limit of the maximum allowed, i.e., less than $6.4 \mathrm{~A}$.

3. Maximize the stability (damping ratio) of $\mathrm{Nc} 1$ with respect to CCS applied at the rotor midspan.

4. Enforce a controller gain roll-off beyond Nc5 to prevent excitation of unmodeled dynamics.

\subsection{Tools for Synthesis}

\subsubsection{Structured Singular Value Framework}

A key result in the robust stability analysis of linear and nonlinear dynamical systems and a foundation of the structured singular value framework is the small gain theorem, stated simply "if the product of the incremental gains of internally stable systems in a feedback loop is strictly less than unity, the feedback loop is also internally stable" (Green 


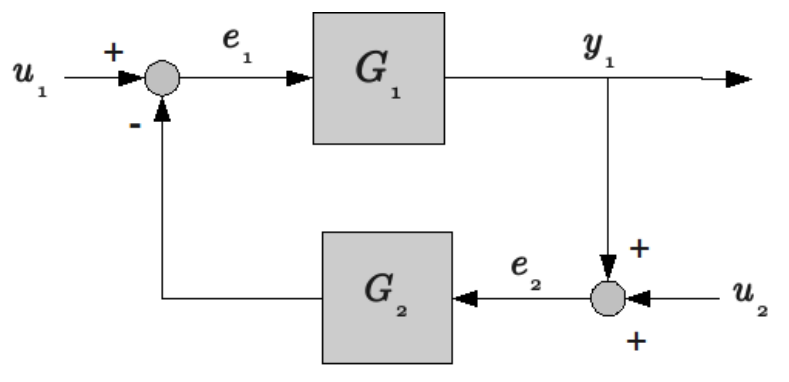

Figure 5.1: Feedback connection illustrating the small gain theorem.

\& Limebeer, 1995). A feedback connection of two input-output stable systems $G_{1}$ and $G_{2}$ is shown in Figure 5.1. We can consider the systems to have finite system gains $\gamma_{1}$ and $\gamma_{2}$, respectively. From the theorem a sufficient condition for the outputs $y_{1}$ and $y_{2}$ to be bounded for any pair of bounded inputs $u_{1}$ and $u_{2}$, is $\gamma_{1} \gamma_{2}<1$. The small gain theorem is useful in robust stability analysis as we are able to characterize $G_{1}$ as a nominal stabilized plant and $G_{2}$ as a feedback connection representing model uncertainty and quantify amount of uncertainty required to destabilize the system (Khalil, 2001).

The $H_{\infty}$ norm, denoted $\|\cdot\|_{\infty}$, provides a measure of the worst-case system gain with finite energy ( $L_{2}$-norm bounded) inputs and outputs and provides a natural framework for control problems involving model uncertainty. The robustness property follows directly from aim of the control law to minimize the worst-case system gain, and by so doing increase the robustness of the closed-loop system to changes in its parameters. $T_{z w}(j \omega)$ is the open-loop transfer function from the exogenous disturbance inputs $w$ to performance metrics $z$. Figure 5.2 shows the standard closed-loop feedback form representing a plant $P$ and controller $K$. In the figure, $u$ is the vector of control actuator signals and $y$ is a vector of measured outputs. The optimal $H_{\infty}$ control problem is to solve for all $K(j \omega)$ that will stabilize the closed-loop system and minimize $\left\|T_{z w}\right\|_{\infty}$. A state-space solution for the optimal $H_{\infty}$ controller was first presented by Doyle et al. (1989). In general this solution is not unique, and it is difficult to obtain the controller that gives the absolute minimum $\left\|T_{z w}\right\|_{\infty}$. However, the suboptimal solution can be specified to find the least upper bound, the supremum, $\gamma$. The supremum is often close to this minimum and the suboptimal $H_{\infty}$ 


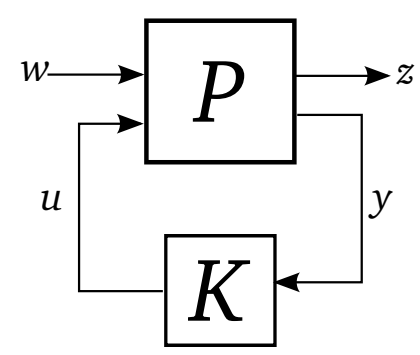

Figure 5.2: Standard plant-controller feedback connection for robust control.

cost can be represented as the maximum singular value of the transfer function matrix $T_{z w}$ for $\gamma>0$

$$
\left\|T_{z w}\right\|_{\infty}:=\bar{\sigma}\left(T_{z w}\right)=\sup _{\omega} \sigma_{\max }\left(T_{z w}(j \omega)\right)<\gamma
$$

With the introduction of unstructured uncertainty in the form of frequency domain weights augmenting the nominal plant model, finding the controller that minimizes the $H_{\infty}$ norm bound yields robust stability and performance. However, the bounded norm minimization is unable to guarantee robustness when structured uncertainty is used in the plant model (Doyle, 1982; Stein \& Doyle, 1991). To solve the structured uncertainty problem requires a new means of describing uncertainty in the plant model. The structured complex uncertainty matrix which defines the set of allowable permutations of the uncertain dynamics is

$$
\Delta_{m}:=\left\{\operatorname{diag}\left[\delta_{1}, \delta_{2}, \ldots, \delta_{k}\right]: \delta_{i} \in \mathbb{C}\right\}
$$

where the scalars $\delta_{i}$ are perturbation in the complex plane representing the parametric uncertainties to be defined in the next section. It is useful to define norm bounded sets of $B \Delta_{m}$ as

$$
B \Delta_{m}:=\left\{\Delta_{m} \in \Delta_{m}: \bar{\sigma}\left(\Delta_{m}\right) \leq 1\right\}
$$

Linear fractional transformations (LFT) provide a generalization of the feedback connection between dynamic elements such as components of the plant model, uncertainty descriptions, and the controller description. An upper-LFT $\left(\mathscr{F}_{u}\right)$ defines the feedback connection between the nominal plant dynamics $G(s)$, given by Equation (4.27), and the un- 


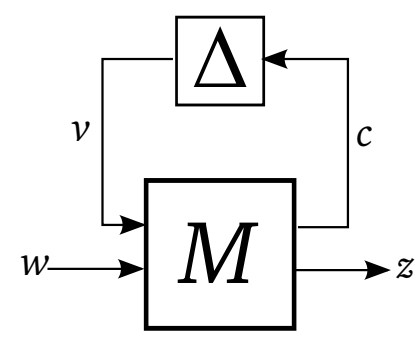

Figure 5.3: Standard form for robust control.

certainty description $\Delta$ as $P(s):=\mathscr{F}_{u}\left(G, \Delta_{m}\right)$. The system $P(s)$ is $2 \times 2$ block structure and contains a complete description of the uncertain plant model to be used for the synthesis of robustly stabilizing controllers. A lower-LFT is used to define the feedback connection between $G(s)$ and a candidate robust controller $K(s)$, as $M(s):=\mathscr{F}_{1}(G, K)$. The system $M(s)$ also has a $2 \times 2$ block structure and is used for the robustness analysis of a specified controller to perturbations within $\Delta$

$$
M \triangleq \mathcal{F}_{l}\left(G_{\mathrm{nom}}, K\right)=\left[\begin{array}{cc}
M_{11} & M_{12} \\
M_{21} & M_{22}
\end{array}\right]
$$

Figure 5.3 shows the interconnections associated with $\Delta$ and $M$, where $w$ and $z$ are exogenous disturbance and performance output signals, respectively, while $v$ and $c$ are signals that interact with the uncertainty description.

Before commencing the control design procedure, it is necessary to formalize the definitions of robustness with respect to stability and performance of the closed-loop system. Nominal stability (NS) is guaranteed by the small gain theorem as long as $M$ is internally stable, i.e., all eigenvalues of $M$ are strictly in the LHP (Zhou et al., 1996). Considering only norm bounded uncertainties, from the small gain theorem a necessary and sufficient condition for robust stability (RS) is the system gain from the bounded energy signals $v$ to $c$ which interact with the $\Delta$ is less than unity

$$
\left\|T_{c v}\right\|_{\infty}:=\left\|M_{11}(j \omega)\right\|_{\infty}<1 \forall \omega,\left\|\Delta_{m}\right\|_{\infty}<1 \text { and NS. }
$$


A necessary and sufficient condition for nominal performance (NP) is the system gain from the bounded energy disturbance input $w$ to the performance output $z$ being less than unity

$$
\left\|T_{z w}\right\|_{\infty}:=\left\|M_{22}(j \omega)\right\|_{\infty}<1 \forall \omega \text { and NS. }
$$

Synthesis of controllers satisfying the NS, NP and RS requirements is achieved using direct application of the sub-optimal $H_{\infty}$ solution given by Equation (5.1), which may be solved with the Matlab function hinfsyn. Solving the robust performance (RP) problem requires the introduction of a fictitious performance block $\Delta_{p}$, producing a combined uncertainty description of

$$
\Delta \in \Delta:=\left\{\operatorname{diag}\left\{\Delta_{m}, \Delta_{p}\right\}: \Delta_{m} \in B \Delta,\left\|\Delta_{p}\right\|_{\infty} \leq 1\right\}
$$

Therefore, a necessary and sufficient condition for RP is

$$
\mu_{\Delta}[M(j \omega)]<1 \forall \omega \text { and } \mathrm{NS}
$$

where the structured singular value for the specified uncertainty $\mu_{\Delta}$ is a positive real function with definition (Doyle, 1982)

$$
\mu_{\Delta}(M):=\left(\min _{\Delta \in \Delta}\{\bar{\sigma}(\Delta): \operatorname{det}(I-\Delta M)=0\}\right)^{-1}
$$

where the reciprocal of $\mu_{\Delta}(M)$ indicates the amount the uncertainty description can be scaled before the closed-loop system $M \Delta$ is no longer stable. The kernel of structured singular value theory is the determinant of the return difference matrix $\operatorname{det}(I-\Delta M)$ which originates from the multivariable Nyquist stability criterion (Bates \& Postlethwaite, 2002). 


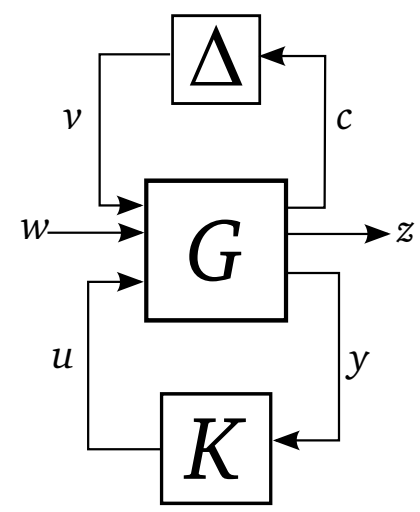

Figure 5.4: $\Delta-G-K$ feedback connection used during synthesis.

\subsubsection{D-K iteration (Complex $\mu-$ Synthesis)}

Finding the stabilizing controller $K$ to solve the RP problem requires the computation of $\mu_{\Delta}(M)$, which is a nonconvex optimization problem (Zhou et al., 1996)

$$
\inf _{K(s)} \sup _{\omega \in \mathcal{R}} \mu_{\Delta}[M(G, K)(j \omega)]<\gamma
$$

The realization that for a complex matrix $M, \mu(M)$ is bounded from below and above by the spectral radius and maximum singular value, respectively,

$$
\rho(M) \leq \mu(M) \leq \bar{\sigma}(M)
$$

The above lower and upper bounds are not useful for computation since they may be arbitrarily large (Gu et al., 2005). However, by introducing the set $\mathcal{U}$ of block-diagonal unitary matrices and the set $\mathcal{D}$ of block-diagonal complex matrices which commute with $\Delta$. Then for any $\Delta \in \Delta$

$$
\begin{aligned}
& U \in \mathcal{U} \rightarrow \bar{\sigma}(U \Delta)=\bar{\sigma}(\Delta), \\
& D \in \mathcal{D} \rightarrow D^{-1} \Delta D=\Delta,
\end{aligned}
$$


and from the definition of $\mu$

$$
\begin{aligned}
& U \in \mathcal{U} \rightarrow \mu(M U)=\mu(M), \\
& D \in \mathcal{D} \rightarrow \mu\left(D M D^{-1}\right)=\mu(M)
\end{aligned}
$$

The bounds in Equation (5.11) can be restated taking advantage of a stable, minimum phase scaling matrix $D$ to provide a more computationally appropriate form

$$
\sup _{U \in \mathcal{U}} \rho(M U) \leq \mu(M) \leq \inf _{D \in \mathcal{D}} \bar{\sigma}\left(D M D^{-1}\right)
$$

Finding a tight upper bound to $\mu(M)$ is then reduced to an optimal diagonal scaling problem performed over a defined grid of frequencies $\omega \in \mathbb{R}$

$$
\inf _{\mathbf{K}(\mathbf{s})} \sup _{\omega \in \mathbb{R}} \inf _{D \in \mathcal{D}} \bar{\sigma}\left\|D M D^{-1}(j \omega)\right\|_{\infty}<\gamma
$$

which can be evaluated by an iterative process known as D-K iteration.

1. Initialization: $D=\mathrm{I}$.

2. $K$-iteration : Find the $H_{\infty}$ controller $K(j \omega)$ to minimize $\left\|D M D^{-1}(j \omega)\right\|_{\infty}$ for a fixed $D$. If $\left\|D M D^{-1}(j \omega)\right\|_{\infty}<1$ or no longer decreases from one iteration to the next, halt the iteration and use the controller with the lowest norm.

3. D-iteration: Find $D(j \omega)$ to minimize over all $\omega$ the value of $\bar{\sigma}\left(D M D^{-1}(j \omega)\right)$ for a fixed $K(j \omega)$ ( $\mu$-analysis step).

4. Fit the magnitude of elements of $D(j \omega)$ to a minimum-phase stable transfer function through interpolation and continue to Step 2.

Steps 2 and 3 of the D-K iteration are each convex operations as they involve the computation of the $H_{\infty}$ norm, for instance using the algebraic Riccati equation approach (Doyle 
et al., 1989). However, the steps are not jointly convex so it is possible that no global optimum exists. In the majority of practical applications convergence is typically achieved. Convergence may not occur in instances of high model order or poor numerical conditioning of the plant, uncertainty models or performance weights.

\subsection{Uncertainty Models}

The mathematical models used for control system analysis and design are, by construction, simplified images of the physical reality (Mackenroth, 2004). Whether a plant model is developed from first principles or through system identification, assumptions are introduced to provide a model which can be analyzed using the tools available to the controls practitioner. The primary benefit of feedback control is the reduction of the effect of plant model uncertainty on closed-loop performance. Large enough deviations in plant parameters will result in a deterioration of the closed-loop performance. In the case of open-loop unstable

plants such as rotor-AMB systems, the controller is both locally stable and operationally critical (Stein, 2003). Therefore, plant parameter deviations need to be scrutinized in more detail to ensure safe operation. Furthermore, the multivariable parameter varying nonlinear nature of rotor-AMB systems makes stabilization more difficult. The primary nonlinearity arises from the square and inverse-square dependence of the magnetic actuator force on the actuator current and air gap, respectively. Bias current linearization and opposingly driven actuator pairs are typically used to overcome this nonlinear effect by considering motion of the rotor about a single equilibrium position (Traxler \& Maslen, 2009). Parameter variations may arise from the speed-dependent gyroscopic effects or load-dependent cross-coupled stiffness effects which both cause system eigenvalues to change. The MIMO nature of the system introduces coupling in the dynamics of the control channels making the task of producing a stabilizing controller a challenge. The development of the plant model should include a nominal plant characterized by its proximity to the equilibrium points of 
the linearized system or average values of its parameters. The $v$-gap metric (see Appendix B) is introduced to quantify the distance between two open-loop plant models based on their closed-loop behavior with the same controller. To complete the description of the system for robust control design this nominal plant model can then be augmented with a description of the perturbations from the nominal model. This augmentation may take several forms which are elaborated in the next section. It is important to produce as concise uncertainty model as possible, to avoid conservatism and maximize the performance of the closed-loop system.

\subsubsection{Structured or Unstructured?}

Model uncertainty may be divided into either structured (parametric) or unstructured (nonparametric) descriptions (Skogestad \& Postlethwaite, 2005). Structured uncertainties are useful when the variation of a known parameter in the model is understood. For example mass variations in a spring-mass-damper system defined, e.g., by a nominal value and $\pm 20 \%$ variation. Unstructured uncertainties are used to characterize the presence of unmodeled or poorly modeled dynamics in the input-output response of a plant. High frequency modes in mechanical systems, e.g., are often deliberately neglected to maintain low model order. Another common example are time delays introduced by a digital controller or which are an intrinsic part of the physics of the plant. As the delays are difficult to explicitly model, a frequency bound may be used to denote phase lags are high frequency.

To include the effects of unstructured uncertainties on a system, complex frequencydependent perturbations $\Delta(s)$ are defined and augmented to the nominal plant $G_{\text {nom }}(s)$ to represent the range of possible responses. Examples such as additive and multiplicative uncertainties are defined by $G_{p}=G_{\mathrm{nom}}(s)+\Delta(s)$ and $G_{p}(s)=G_{\mathrm{nom}}(s)(1+\Delta(s))$, respectively. The lack of specificity is one drawback of modeling using unstructured uncertainty and in many cases this leads to conservative controller performance. Furthermore, using unstructured uncertainty to bound variations in multiple natural frequencies of a system 
will require high order uncertainty weighting functions (Steinbuch et al., 1998).

For the MBTRI plant, an extensive modeling and model validation effort preceded control design, thus we were confident with the accuracy of the nominal model (see Section 4.4 on page 82) for low-speed operation in the absence of CCS. Exciting high frequency unmodeled dynamics can be avoided through suitable control gain roll-off above the third bending mode $\mathrm{Nc} 3(980 \mathrm{~Hz})$. Low frequency neglected dynamics can be an issue as the MBTRI test rig has foundation dynamics, i.e., vibration of the bearing pedestals and concrete foundation, visible from the experimental transfer function plots (artifacts around 350 $\mathrm{Hz}$ in Figure 4.24). However, so long as these modes are far from the bending modes, or beyond the gain crossover frequency, or sufficiently damped they do not pose a problem. Therefore, unstructured uncertainty descriptions were not used. Li (2006) listed several parameters in rotor-AMB systems which when perturbed will strongly affect the closed-loop stability and performance. Uncertainty in the AMB parameters, $K_{x}$ and $K_{i}$, is well managed by feedback so long as the minimum controller bandwidth exceeds the unstable poles. The effect of destabilizing cross-coupled stiffness and the high speed operation of the test rig each produced large enough deviations from the nominal plant description that stability could no longer be guaranteed. Therefore, structured uncertainty models are exclusively adopted.

\subsubsection{Uncertainty Model For Supercritical Operation}

The closed-loop performance of flexible structures, such as AMBs supporting flexible rotors, is most sensitive to variations in the frequency of the lightly damped bending modes. Though the modal damping levels are also uncertain, variations in modal damping have a smaller effect on the closed-loop performance (Balas \& Young, 1995). The Campbell diagram in Figure 4.9 plots the rotor eigenvalues as a function of rotating speed and was discussed during the rotordynamic analysis of the MBTRI rotor (see Section 4.2.1.1 on page 61). From Equation (4.8), the effect of a non-zero operating speed $\Omega$ is to couple the 
dynamics of the $x$ and $y$ channels and cause each rotor mode to split into a forward and backward component. This uncertainty is modeled in two ways:

1. by defining $\Omega$ as an uncertain real parameter varying between 0 and $1885 \mathrm{rad} / \mathrm{s}$ (18,000 rpm) denoted Speed Model 1, or

2. by defining the eigenvalues of rotor modes affected by the gyroscopics with uncertain natural frequencies indicated by trajectories in the Campbell diagram and denoted Speed Model 2.

Speed Model 1 represents a direct way to capture the effect of operating speed on the rotordynamics. Limitations of the Matlab ureal function require the nominal value of $\Omega$ to be close to the the midpoint of the upper and lower limits of the uncertainty range, i.e., 943 $\mathrm{rad} / \mathrm{s}$. The uncertain state-space model (uss) created is fully coupled along all four control axes. Since the model validation focused on building an accurate model at rest (blockdiagonal model since $x \leftrightarrow y$ cross-coupled gains are small, but $x \leftrightarrow x$ and $y \leftrightarrow y$ are larger), having full $4 \times 4$ coupling produced a nominal model that was difficult to experimentally validate. Modeling the speed variation directly increased the complexity of the model, preventing the synthesis of a high performance robust controller as we shall see later on. However, this was not expected as the v-gap of Speed Model 1 is reasonable as compared to the other plant models shown in Table 5.1.

Speed Model 2 models the effect of the speed variation on the rotor model, i.e., using eigenvalue perturbations, rather than the speed variation itself. This phenomenological approach is advantageous since the already validated nominal plant model can be extended to represent the dynamics over an arbitrary speed range without introducing the coupling between channels. The first and second rotor bending modes (Nc3 and Nc4) exhibit a stronger gyroscopic splitting than the rigid body modes, and real-valued parametric uncertainty was used to capture this variation. The nominal frequency for Nc3 is $223 \mathrm{~Hz}$ and range of variation is $213-230 \mathrm{~Hz}$, while the nominal frequency for $\mathrm{Nc} 4$ is $548 \mathrm{~Hz}$, and the modeled variation is $540-552 \mathrm{~Hz}$. This modeling was accomplished using the Matlab uncertain elements, i.e., ureal ('f_Nc3',223,'Range', [213 230]), and ureal ('f_Nc4', 548,'Range', [540 
552]).

Uncertainty in the natural frequency of the third bending mode Nc5 was ignored as it beyond the operating speed. Furthermore, since Nc5 is outside the controller bandwidth it can be stabilized by gain roll-off. The Campbell diagram was used as a starting point for the uncertainty ranges which were refined experimentally. The uncertain modal state matrix is based on the realization of the second-order transfer function response for the $i$-th bending mode in a single plane can be expressed as

$$
\hat{A}_{i}=\left[\begin{array}{cc}
0 & 1 \\
-\omega_{i}^{2} & -2 \zeta \omega_{i}
\end{array}\right]+\left[\begin{array}{l}
0 \\
1
\end{array}\right] \delta_{i}\left[\begin{array}{cc}
2 \omega_{i}^{2} & 2 \zeta \omega_{i}
\end{array}\right]
$$

where $\omega_{i}$ is the natural frequency, $\zeta_{i}$ is the damping of the mode. The result holds as long as the uncertainty $\delta_{i}$ in the two natural frequencies is small (Balas \& Doyle, 1994). When assembling a complete two-plane rotor model, i.e., dynamics along both $x$ and $y$ axes, the same uncertainties $\delta_{1}$ and $\delta_{2}$ are used for both axes. The use of repeated uncertainty blocks serves to reduce conservatism in the model by eliminating the duplicate uncertainty descriptions. Figure 5.5 shows the uncertain plant dynamics in the form of singular value plot. The portion of the frequency close first and second bending modes has denser lines indicating the effect of the uncertainty. As intended, the low frequency region and the overall magnitude of the singular values are hardly different from the nominal dynamics presented in Figure 4.21. As a result, we can be sure that the diagonal dominance of the plant model persists. The v-gap metric for Speed Model 2 is 0.656 which is slightly greater than the other uncertainty candidate but still reasonable. However, in the upcoming synthesis section Speed Model 2, by virtues of its reduced conservatism, will greatly exceed the robust performance is Speed Model 1. 
Table 5.1: v-gap metric of uncertainty models for supercritical operation with respect to the nominal plant model.

\begin{tabular}{|c|c|}
\hline & v-gap \\
\hline \hline Nominal model w/ $\Omega=9,000 \mathrm{rpm}$ & 0.596 \\
\hline Nominal model w/ $\Omega=18,000 \mathrm{rpm}$ & 0.608 \\
\hline Speed Model 1 & 0.608 \\
\hline Speed Model 2 & 0.656 \\
\hline
\end{tabular}

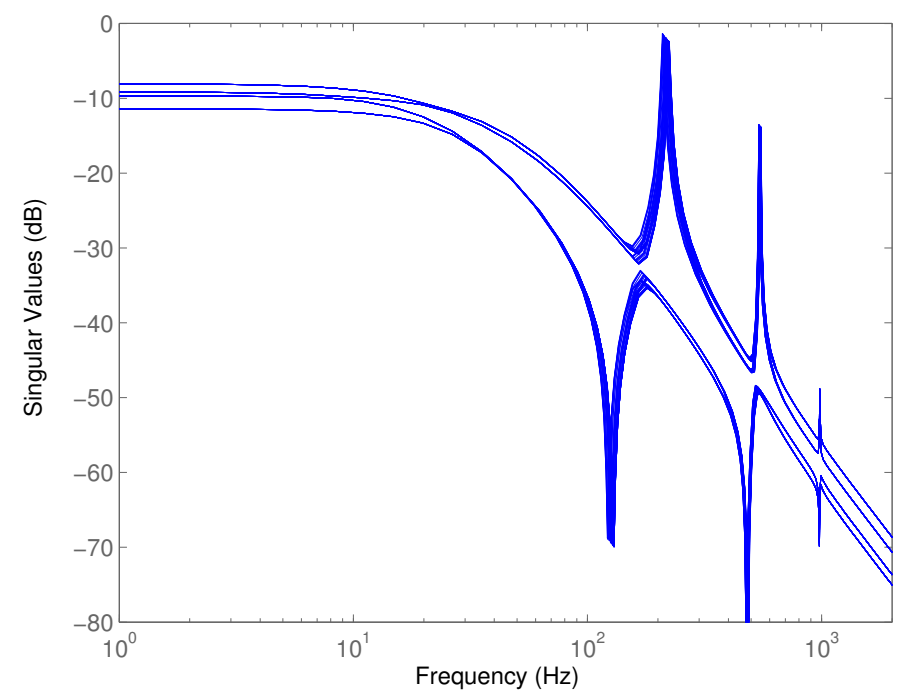

Figure 5.5: Singular value plot of uncertain plant model illustrating variation in Nc3 and $\mathrm{Nc} 4$ natural frequencies. Compare with nominal plant model in Figure 4.21. 


\subsubsection{Uncertainty Model For Enhancing Stability Threshold}

As with the gyroscopic effect, modeling of the impact of CCS on the open-loop plant dynamics can be carried out directly from first principles or phenomenologically. In the case of the former, uncertain CCS is modeled directly as a real-valued stiffness varying between 0 and $4 \mathrm{MN} / \mathrm{m}$ acting at the rotor midspan. Rotor station \#23 in the finite element model corresponds to the midspan location. Therefore, the associated rotor displacements as shown in Equation (4.1), are $x_{23}$ and $y_{23}$. Assuming the CCS is the sole exogenous disturbance in the system

$$
B_{\mathrm{w}} F_{\mathrm{w}}=Q\left[\begin{array}{cc}
0 & 1 \\
-1 & 0
\end{array}\right]\left[\begin{array}{l}
q_{x_{23}} \\
q_{y_{23}}
\end{array}\right]
$$

where the stiffness $Q$ can be modeled in two ways:

1. as a real parameter varying from 0 to $1.8 \mathrm{MN} / \mathrm{m}$ with a nominal value of $0.9 \mathrm{MN} / \mathrm{m}$ denoted CCS Model 1, or

2. as a real parameter varying from 0 to $2.4 \mathrm{MN} / \mathrm{m}$ with a nominal value of $1.2 \mathrm{MN} / \mathrm{m}$ denoted CCS Model 2.

The resulting model shows, as expected, increased coupling between the $x$ and $y$ lateral rotor displacements at all rotating speeds. Figures 5.6 and 5.7 demonstrate the coupling with four Bode plots and a singular value plot of the nominal plant augmented with the uncertainty defined by CCS Model 1. While the demonstrated coupling between the axes is mathematically correct, the additional dynamics complicate the model and presents a open-loop plant that is more of a challenge to validate and control. This observation was echoed by a $v$-gap metric of unity between the nominal plant and a plant augmented by CCS Model 1 (see Table 5.3). Increased conservatism arises as $Q$ affects several parameters within the plant model. While the effect on the Nc1 pole is important and the most desired to be captured by this uncertainty definition, several collateral effects occur such as the 


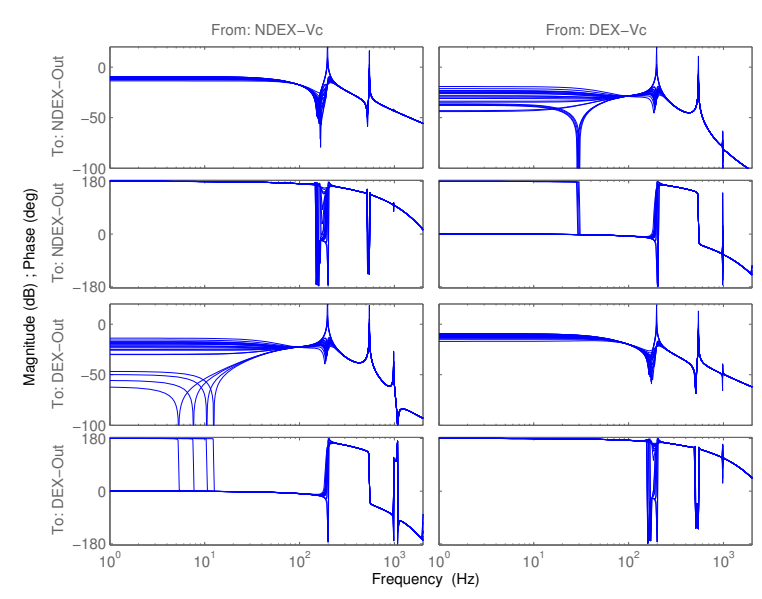

(a) $12-12$

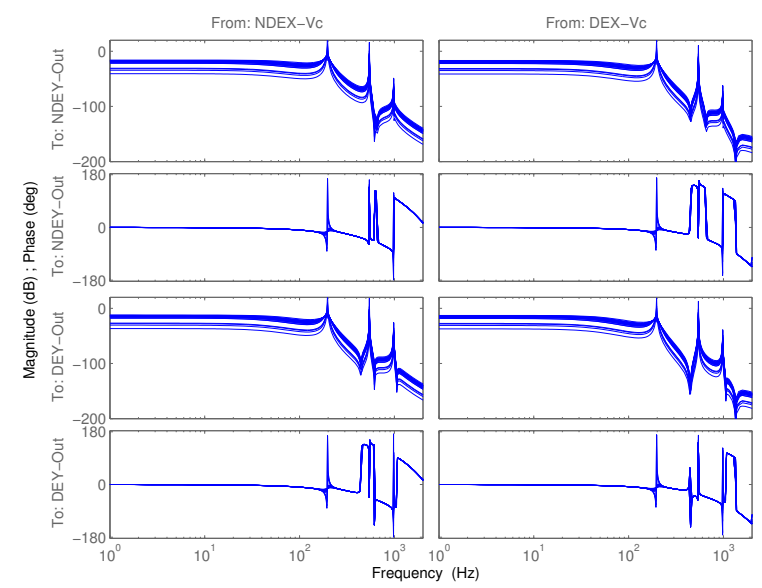

(c) $34-12$

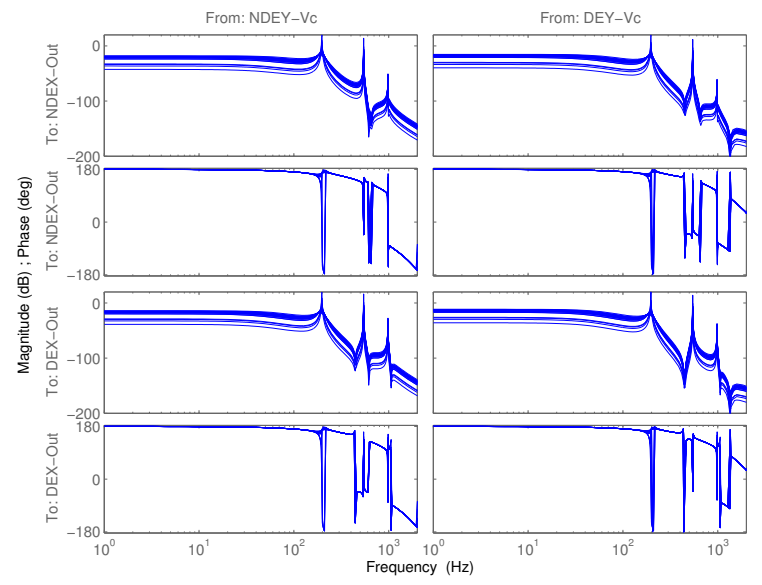

(b) $12-34$

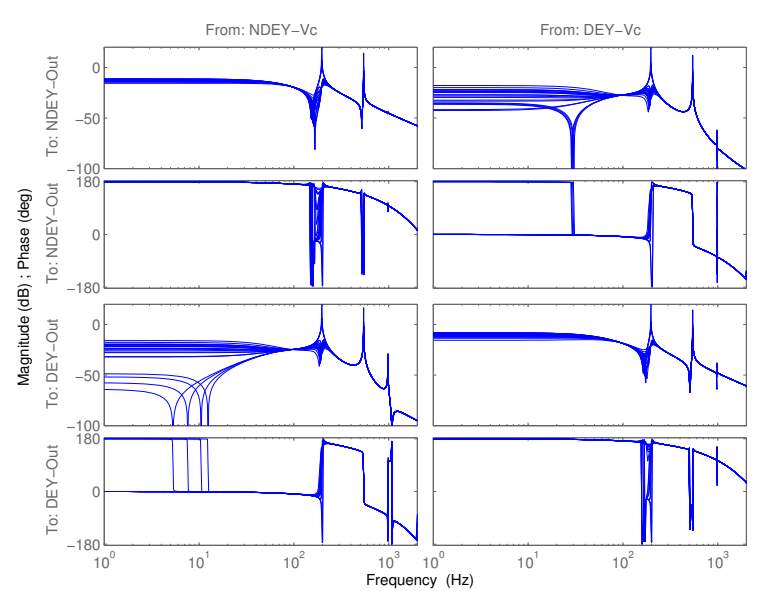

(d) 34-34

Figure 5.6: Bode plots of plant with uncertainty defined by CCS Model 1.

perturbation of $\mathrm{Nc} 3$ and $\mathrm{Nc} 4$ poles and zeros are not desired. CCS Model 2 has an identical structure to CCS Model 1 with the only difference being an increased nominal value and upper limit of $Q$. The $v$-gap of CCS Model 2 is also unity.

Phenomenological modeling of the uncertainty attempts to model the empirical effect of the CCS on the plant rather than the intrinsic dynamics of the rotor-AMB system which have been shown to be highly conservative by CCS Models 1 and 2. By far the most significant effect of CCS on the plant is the trajectory of the RHP pole of Nc1 as CCS is varied (see Figure 3.6b and Table 5.2). Increasing the CCS has the effect of splitting the 


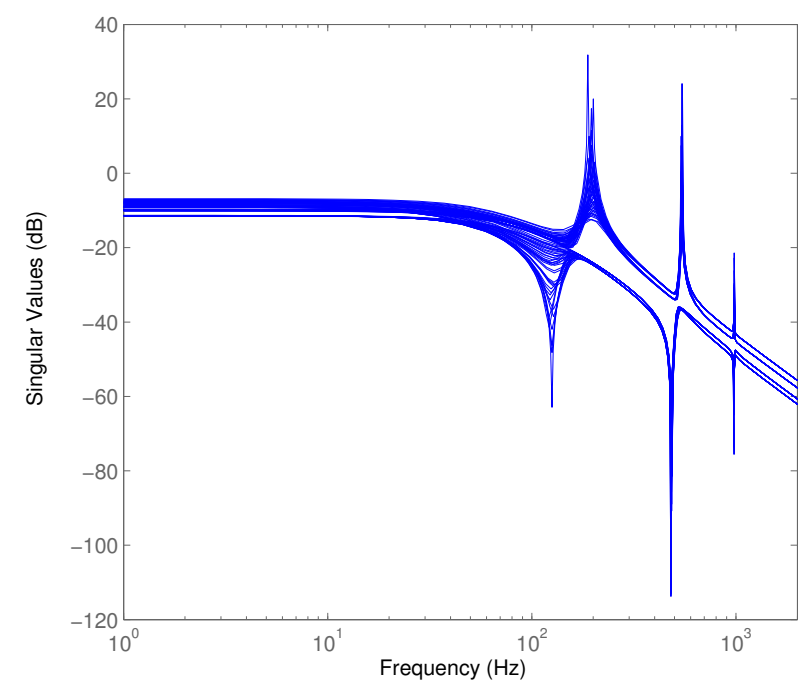

Figure 5.7: Singular value plot of plant with uncertainty defined by CCS Model 1.

real RHP pole due to $\mathrm{Nc} 1$ into a complex pole that moves further to the right of the complex plane. Assuming the Nc1 complex eigenvalue (an RHP pole) of the form $\sigma \pm j \omega$, the effect of CCS on $\sigma$ can be modeled either:

1. as a real-valued uncertainty varying from 200 to $240 \mathrm{rad} / \mathrm{s}$ with a nominal value of $220 \mathrm{rad} / \mathrm{s}$ denoted CCS Model 3, or

2. as a real-valued uncertainty varying from 200 to $260 \mathrm{rad} / \mathrm{s}$ with a nominal value of $230 \mathrm{rad} / \mathrm{s}$ denoted CCS Model 4.

An eigenvalue perturbation description similar to Equation (5.18) was used to represent the variations above, and combine into the state-space description of the rotor-AMB system. In both cases, no additional $x, y$ coupling is generated in the model (as evidenced by empty off-diagonal response in Figure 5.8), even though the same destabilizing effect on Nc1 is produced as CCS Model 1. The advantages of the phenomenological approach are that $x, y$ coupling (off-diagonal gain) is minimized, allowing the nominal model developed and validated in Chapter 4 to be extended for use. The singular value plot of the uncertain plant with CCS Model 3 is shown in Figure 5.9. From this plot it is again clear that effects of 
Table 5.2: The effect of varying CCS magnitude on the location of Nc1 eigenvalue.

\begin{tabular}{|c|c|c|c|}
\hline $\mathrm{Q}(\mathrm{N} / \mathrm{m})$ & Eigenvalue $^{\dagger}$ & Natural frequency & Damping ratio \\
\hline \hline 0 & 195 & $31 \mathrm{~Hz}$ & -1 \\
\hline $5 \mathrm{e} 5$ & $+199 \pm j 55.7$ & $32.9 \mathrm{~Hz}$ & 0.963 \\
\hline $10 \mathrm{e} 5$ & $+199 \pm j 55.7$ & $32.9 \mathrm{~Hz}$ & -0.963 \\
\hline $15 \mathrm{e} 5$ & $+207 \pm j 80.7$ & $35.5 \mathrm{~Hz}$ & -0.928 \\
\hline $20 \mathrm{e} 5$ & $+217 \pm j 104$ & $38.2 \mathrm{~Hz}$ & -0.904 \\
\hline $25 \mathrm{e} 5$ & $+227 \pm j 125$ & $39.8 \mathrm{~Hz}$ & -0.908 \\
\hline $30 \mathrm{e} 5$ & $+237 \pm j 144$ & $44.1 \mathrm{~Hz}$ & -0.855 \\
\hline $40 \mathrm{e} 5$ & $-256 \pm j 180$ & $49.9 \mathrm{~Hz}$ & -0.818 \\
\hline
\end{tabular}

$\dagger$ Only RHP pole shown, a conjugate exists on LHP

Table 5.3: v-gap metric of uncertainty models for cross-coupled stiffness variation with respect to the nominal plant model.

\begin{tabular}{|c|c|}
\hline Model compared with nominal plant & v-gap \\
\hline \hline CCS Model 1 & 1 \\
\hline CCS Model 2 & 1 \\
\hline CCS Model 3 & 0.624 \\
\hline CCS Model 4 & 0.624 \\
\hline
\end{tabular}

the uncertain CCS modeled through a single eigenvalue perturbation remain largely in the low frequency band around Nc1. The uncertainty set described by CCS Model 3 and 4 is thus more compact than that of CCS Model 1 and 2 for the equivalent physical responses. The gap metrics of CCS Model 3 and CCS Model 4 are identically 0.624 indicating a not unreasonable distance from the nominal plant description.

\subsection{Weighting Function Selection}

Performance specifications are characterized in the frequency domain as typical rotor-AMB systems lack specific time domain specifications, e.g., overshoot and settling time and these do not fit directly into the frequency based robust $H_{\infty}$ and $\mu$ synthesis frameworks (Franchek, 1996). The loop-shaping approach is preferred over the signal based approach as it does not require implementation specific information on the magnitudes of signals in the closed-loop system (van de Wal et al., 2002). Sensitivity functions serve as useful 


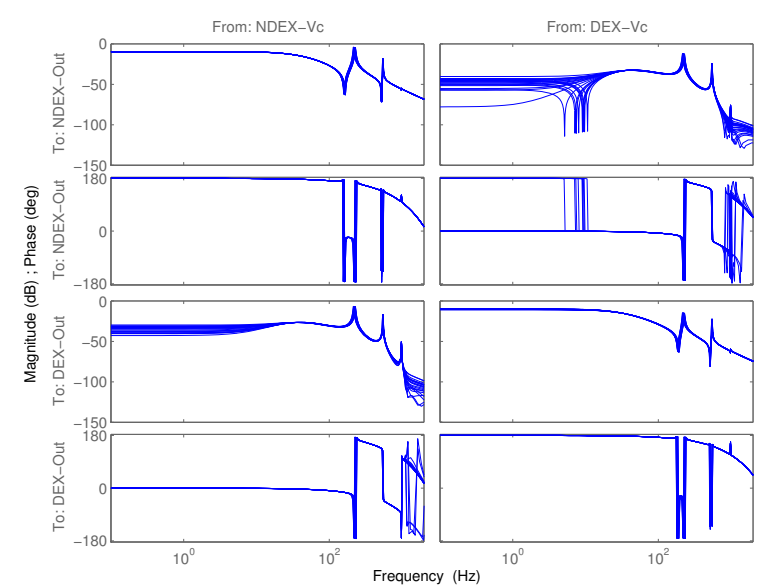

(a) $12-12$

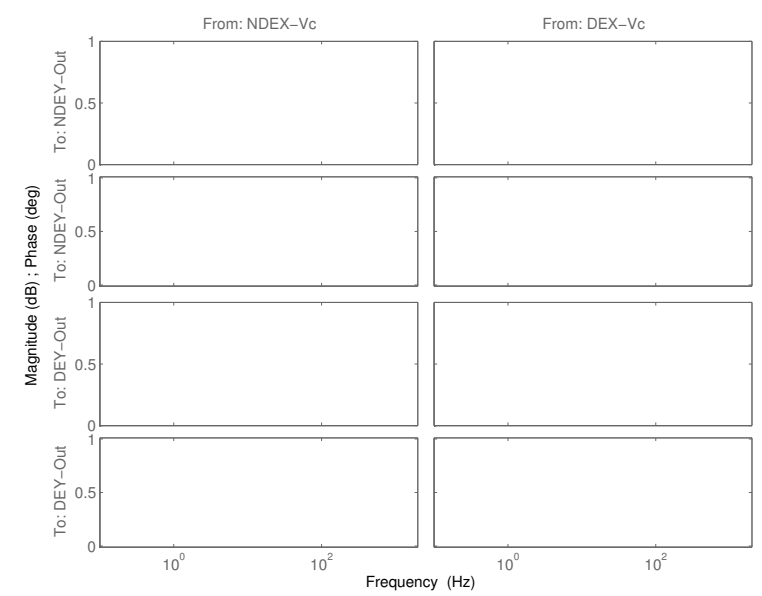

(c) $34-12$

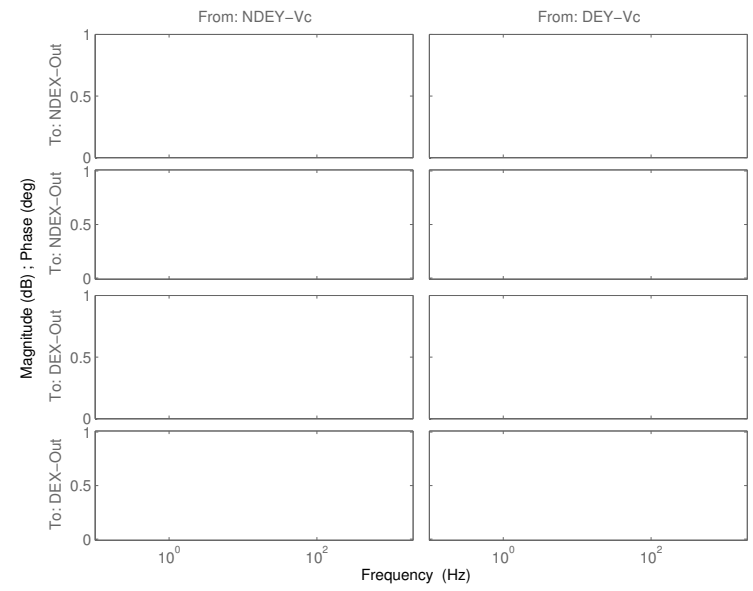

(b) $12-34$

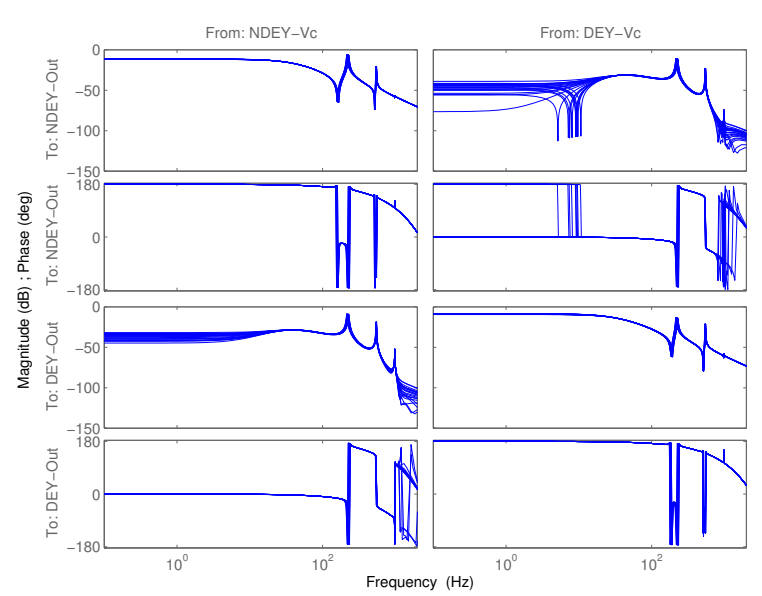

(d) 34-34

Figure 5.8: Bode plots of plant with uncertainty defined by CCS Model 3. 


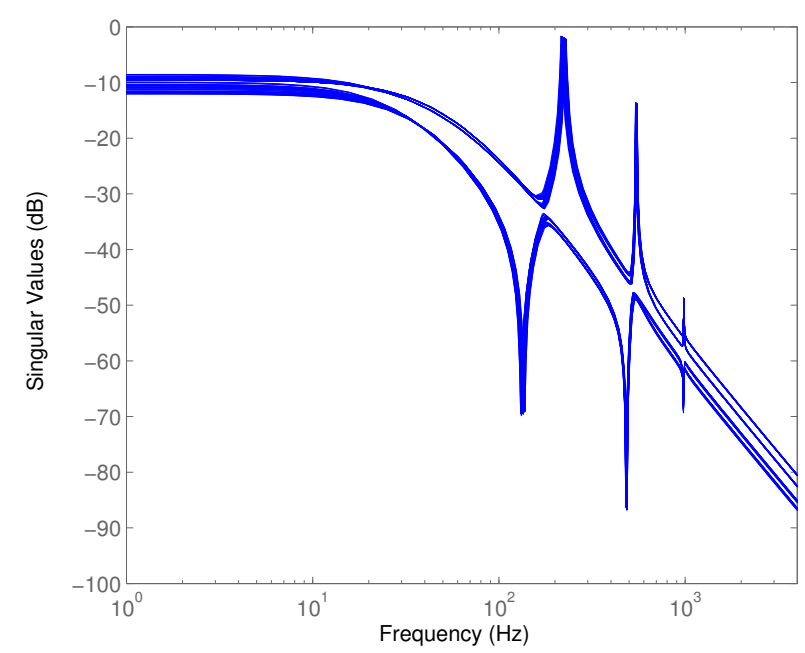

Figure 5.9: Singular value plot of plant with uncertainty defined by CCS Model 3, compare with Figure 5.7.

measures of control performance in the frequency domain. Considering a unity feedback connection of a plant $G(s)$, controller $K(s)$, with exogenous disturbance forces acting on the plant input and output $d_{i}$ and $d_{o}$ respectively, sensor noise $n$ and control effort $u$, controlled variable output $y$ and controller reference input signal $r$. The output variable and the control signal can be represented by

$$
\begin{aligned}
& y(s)=T_{i}(s) r(s)+S_{o}(s) d_{o}(s)+G S_{o}(s) d_{i}(s)-T_{o}(s) n(s), \\
& u(s)=K S_{o}(s) r(s)-K S_{o}(s) d_{o}(s)-S_{i}(s) d_{i}(s)-K S_{o}(s) n(s),
\end{aligned}
$$

where several sensitivity functions can be defined (Larsonneur, 2009):

- Input Sensitivity Function $\left(S_{\mathrm{i}} \triangleq(I+K G)^{-1}\right)$ : a measure of system rejection of disturbances at plant inputs;

- Complementary Input Sensitivity Function $\left(T_{\mathrm{i}} \triangleq K S_{\mathrm{o}} G\right)$ : a measure of the effect of disturbance at the reference input on the control signal;

- Output Sensitivity Function $\left(S_{\mathrm{o}} \triangleq(I+G K)^{-1}\right)$ : a measure of the noise rejection and closed-loop command tracking; 
- Complementary Output Sensitivity Function $\left(T_{\mathrm{o}} \triangleq G S_{\mathrm{i}} K\right)$ : a measure of the effect of noise on the control signal;

- Process Sensitivity Function $\left(G S_{\mathrm{i}}\right)$ : a measure of the closed loop mechanical compliance (reciprocal of stiffness) of the rotor-bearing;

- Control Sensitivity Function $\left(K S_{\mathrm{o}}\right)$ : a measure of the control effort.

A bound on a single sensitivity function, such as the output sensitivity takes the form of $\left\|W_{s}(s) S(s)\right\|_{\infty}<1$, where $W_{s}(s)$ is a stable, proper transfer function. If the inequality is satisfied, the inverse weighting function $W_{s}^{-1}(s)$ represents the upper bound on $S(s)$. A typical inverse weighting function to achieve low frequency disturbance attenuation, for example, is shown in Figure 5.10. For high performance motion control systems typified by rotor-AMB applications, a single sensitivity provides insufficient degrees of freedom to simultaneously specify disturbance rejection region while minimizing controller bandwidth and as well as reference tracking performance (Schonhoff et al., 2002). By combining several weighted sensitivity functions, i.e., $\left\|W_{s}(s) S(s)\right\|_{\infty}<1,\left\|W_{u}(s) K S(s)\right\|_{\infty}<1$, and $\left\|W_{r}(s) T(s)\right\|_{\infty}<1$, into a stacked objective

$$
\min _{K}\left\|\begin{array}{c}
W_{s}(s) S(s) \\
W_{u}(s) K S(s) \\
W_{r}(s) T(s)
\end{array}\right\|_{\infty}<\gamma
$$

and letting the $H_{\infty}$ synthesis process handle the trade-offs between competing goals so that maximum performance may be attained.

The four-block problem provides a means for any three non-complimentary functions to be weighted individually. The advantages of the four-block scheme over the two or three block problems are its well-posedness and avoidance of pole-zero cancellation by the synthesized controller (Englehart \& Smith, 1990; Smith, 1990). 


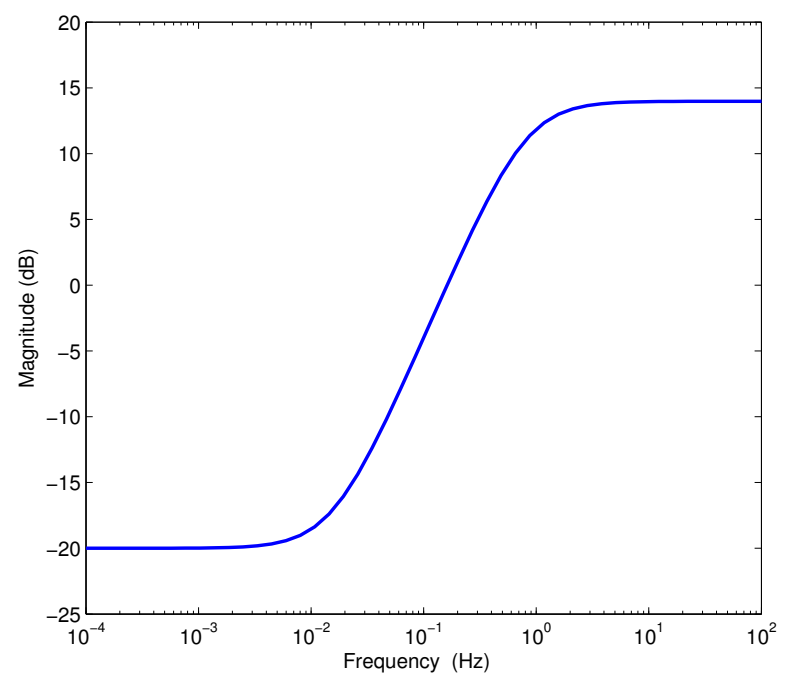

Figure 5.10: Typical inverse performance weighting function, $W_{p}^{-1}(s)$.

Figure 5.11 illustrates the feedback connection of the weighting functions to the plant and controller blocks. The reference input $r$ and disturbance input $d$ are new signals introduced. Shaping the control sensitivity function $K S(s)$ is necessary to effect a controller gain roll-off at high frequencies to avoid exciting unmodeled dynamics or amplifying sensor noise. Shaping the process sensitivity function $G S(s)$ affects the closed-loop damping, while the shaping the output sensitivity $S(s)$ translates directly to modifying the disturbance rejection properties. The complimentary sensitivity $T(s)$ cannot be shaped independently of the aforementioned functions as a result of the complimentary nature of the four sensitivity functions.

The system gain from disturbance inputs to performance outputs is

$$
\left[\begin{array}{l}
z_{1} \\
z_{2}
\end{array}\right]=T_{z w}\left[\begin{array}{l}
d \\
r
\end{array}\right],
$$




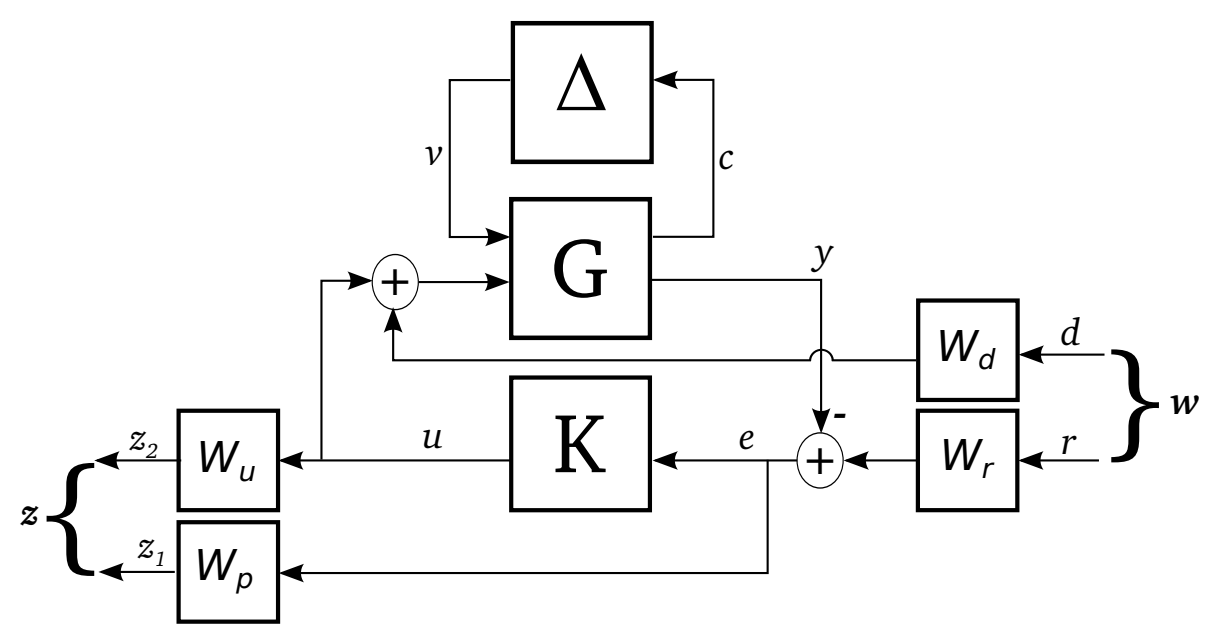

Figure 5.11: Interconnection of performance weights with plant, uncertainty description and controller.

and to guarantee the desired nominal performance specified by the above sensitivity functions, the cost $\gamma$ to be minimized by the controller $K$ must be less than unity

$$
\begin{array}{r}
\min _{K}\left\|T_{z w}\right\|_{\infty}<\gamma, \\
\left\|\left[\begin{array}{rr}
W_{p}(I+G K)^{-1} W_{r} & W_{p} G(I+G K)^{-1} W_{d} \\
W_{u} K(I+G K)^{-1} W_{r} & W_{u} G K(I+G K)^{-1} W_{d}
\end{array}\right]\right\|_{\infty}<\gamma, \\
\left\|\left[\begin{array}{cc}
W_{p} S W_{r} & W_{p} G S W_{d} \\
W_{u} K S W_{r} & W_{u} T W_{d}
\end{array}\right]\right\|_{\infty}<\gamma .
\end{array}
$$

The resulting upper bounds on the $S, G S, K S$ and $T$, are $\left(W_{p} W_{r}\right)^{-1},\left(W_{p} W_{d}\right)^{-1},\left(W_{u} W_{r}\right)^{-1}$ and $\left(W_{u} W_{d}\right)^{-1}$, respectively. The above cost function solves the nominal performance problem. Since $\Delta$ has been defined in block diagonal form, the straightforward extension of Equation (5.23) to solve the robust performance problem is carried out by defining the structured uncertainty set $\Delta$ and finding the controller that minimizes $\mu_{\Delta}(M)$ using D-K iteration. 


\subsubsection{Weighting Function Template}

To enforce a suitable trade-off between the four sensitivity functions whilst maintaining a connection to the physical behavior of the system, a simple template for the weighting functions was adopted (Schonhoff, 2002). During the process of iterating over weighting functions one is liable to lose this connection and create arbitrary weighting functions that mask the reasons behind the success of a given weighting function, or lack thereof. The template is composed of parameters: sensitivity constant $M_{S}$, compliance constant $M_{G S}$, control gain constant $M_{K S}$, control bandwidth constant $\omega_{\mathrm{b}}$, integrator constant $\omega_{\mathrm{i}}$, and control gain roll-off constant $\omega_{L}$. The two dynamic (frequency dependent) weighting functions parametrized by some of the above constants are

$$
\begin{gathered}
W_{p}(s)=K_{1} \frac{0.4 s+\omega_{\mathrm{i}}}{s+K_{2} \omega_{\mathrm{i}}}, \text { and } \\
W_{u}(s)=\frac{M_{S}}{M_{K S}} \cdot \frac{\omega_{1}^{2}}{\omega_{L}^{2}} \cdot \frac{s^{2}+\sqrt{2} \omega_{L}+\omega_{L}^{2}}{s^{2}+\sqrt{2} \omega_{1}+\omega_{1}^{2}},
\end{gathered}
$$

where $\omega_{1}=15 \omega_{L}$ is an additional high-frequency pole included to ensure the inverse weighting function is proper and $K_{1}$ and $K_{2}$ are scalars to tune the gain and low frequency integration produced by $W_{p}$. Most motion control applications have requirements for high static stiffness to minimize steady state error. This objective can be enforced by a $W_{p}(s)$ with a low pass characteristic with high pass-band gain and stop-band attenuation resulting in a significant integrator gain at low frequencies. In contrast, the MBTRI uses a fairly flat proper first-order filter, chosen for simplicity. An optional integrator was placed in parallel with the $\mu$-synthesis for fine grained control over the integration action and support for antiwindup (Iqbal et al., 2010). Increasing $\omega_{1}$ has the added effect of dropping the gain floor of the control signal at high frequency, i.e., enforcing a sharper controller gain roll-off. The remaining weighting functions are scalar gains

$$
W_{r}(s)=\frac{1}{M_{S}}, \text { and }
$$




$$
W_{d}(s)=\frac{1}{M_{G S}} .
$$

Bode magnitude plots of the above functions are shown in Figure 5.12. The weighting functions defined above are either SISO transfer functions or scalar gains. However, the weights in optimization problem of Equation (5.23) are transfer function matrices or constant matrices. The performance requirements and dynamics of the four channels of the rotor-AMB system are similar (minor differences arise from variations in the displacement sensor sensitivity and observed displacement of the bending modes). Therefore, the same weighting functions may be used for each channel giving a weighting function matrix that is typically diagonal. The following weighting function matrices are defined for direct interconnection with the plant model

$$
\begin{gathered}
W_{p}(s)=\operatorname{blkdiag}\left[W_{p, N D E}, W_{p, D E}, W_{p, N D E}, W_{p, D E}\right]:=\left[\begin{array}{c|c}
A_{w, p} & B_{w, p} \\
\hline C_{w, p} & D_{w, p}
\end{array}\right], \\
W_{u}(s)=\operatorname{blkdiag}\left[W_{u, N D E}, W_{u, D E}, W_{u, N D E}, W_{u, D E}\right]:=\left[\begin{array}{c|c}
A_{w, u} & B_{w, u} \\
\hline C_{w, u} & D_{w, u}
\end{array}\right], \\
W_{r}(s):=\operatorname{blkdiag}\left[W_{r, N D E}, W_{r, D E}, W_{r, N D E}, W_{r, D E}\right], \\
W_{d}(s):=\operatorname{blkdiag}\left[W_{d, N D E}, W_{d, D E}, W_{d, N D E}, W_{d, D E}\right] .
\end{gathered}
$$

$W_{p}(s)$ and $W_{u}(s)$ are converted into state-space form to improve the numerical conditioning prior to assembly with the plant model. $W_{r}(s)$ and $W_{d}(s)$ are in the form of scalar matrices. A complete state-space description for the uncertain plant with including the dynamic contribution of performance weighting functions and the modeled uncertainty from the bending modes is 


$$
P(s):=\left\{\begin{array}{c}
{\left[\begin{array}{c}
\dot{x}(t) \\
c(t) \\
z(t) \\
y(t)
\end{array}\right]=\left[\begin{array}{cccc}
A & B_{\Delta} & B_{1} & B_{2} \\
C_{\Delta} & 0 & 0 & 0 \\
C_{1} & 0 & D_{11} & D_{12} \\
C_{2} & 0 & 0 & 0
\end{array}\right]\left[\begin{array}{c}
x(t) \\
v(t) \\
w(t) \\
u(t)
\end{array}\right],} \\
=\Delta c(t)
\end{array}\right.
$$

where

$$
\begin{aligned}
& A=\left[\begin{array}{cccc}
\hat{A}_{r} & 0 & 0 & 0 \\
B_{d, u} C_{r, s} & A_{d} & 0 & 0 \\
-B_{w, p} D_{d} C_{r, s} & -B_{w, p} C_{d} & A_{w, p} & 0 \\
0 & 0 & 0 & A_{w, u}
\end{array}\right] \\
& B_{\Delta}=\left[\begin{array}{lllll}
0 & I_{2} & 0 & I_{2} & 0
\end{array}\right]^{\top} \\
& c_{\Delta}=\left[\begin{array}{ccccc}
0 & -2 \omega_{3}^{2} & 0 & -2 \zeta \omega_{3} & 0 \\
0 & -2 \omega_{4}^{2} & 0 & -2 \zeta \omega_{4} & 0
\end{array}\right] \\
& C_{\Delta}=\left[\begin{array}{ll} 
& \\
c_{\Delta} & c_{\Delta}
\end{array}\right] \\
& B_{1}=\left[\begin{array}{cccc}
\hat{B}_{r} W_{d} & 0 & 0 & 0 \\
0 & 0 & B_{w, p} W_{r} & 0
\end{array}\right]^{\top} \\
& B_{2}=\left[\begin{array}{cccc}
\hat{B}_{r} & 0 & 0 & B_{w, u}
\end{array}\right]^{\mathrm{T}} \\
& C_{1}=\left[\begin{array}{cccc}
-D_{w, p} D_{d} C_{r, s} & -D_{w, p} C_{d} & C_{w, p} & 0 \\
0 & 0 & 0 & C_{w, u}
\end{array}\right] \\
& C_{2}=\left[\begin{array}{llll}
D_{d} C_{r, s} & C_{d} & 0 & 0
\end{array}\right] \\
& D_{11}=\left[\begin{array}{cc}
0 & W_{r} D_{w, p} \\
0 & 0
\end{array}\right], D_{12}=\left[\begin{array}{c}
0 \\
D_{w, u}
\end{array}\right],
\end{aligned}
$$




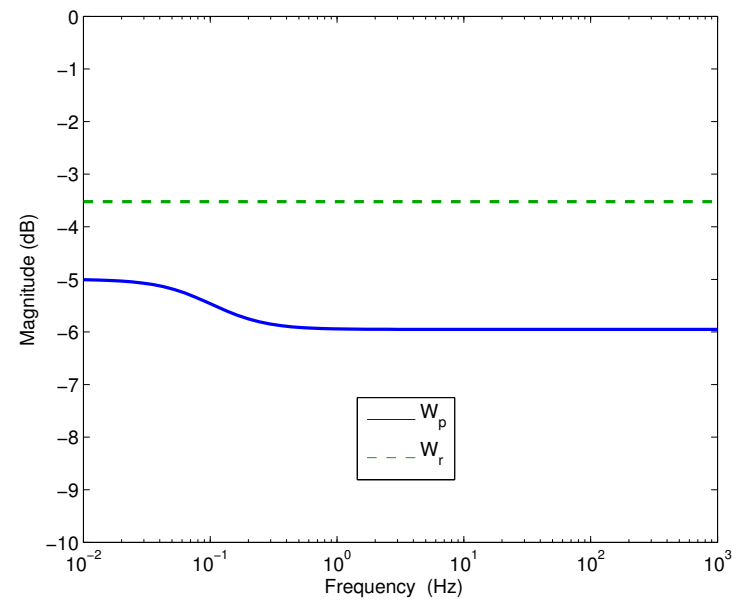

(a) $W_{p}$ and $W_{r}$.

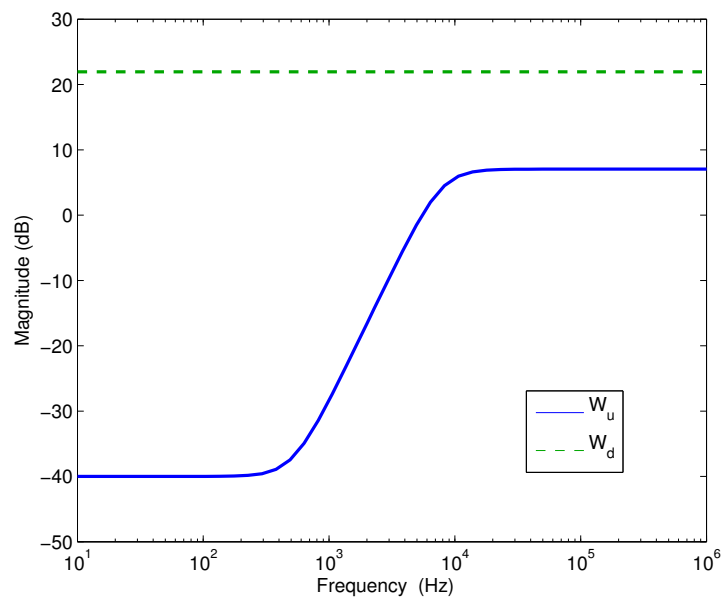

(b) $W_{u}$ and $W_{d}$.

Figure 5.12: Weighting functions used in design template.

and where $\omega_{3}$ and $\omega_{4}$ are the nominal natural frequencies of the first two bending modes $\mathrm{Nc} 3$ and Nc4, and $\zeta=0.2 \%$ is the modal damping. The uncertain state space model $P$ contains 48 states, a contribution of 36 states from rotor-AMB model $G$, and 12 states from the dynamics of $W_{p}(s)$ and $W_{u}(s)$ repeated across four control channels. Balancing using a diagonal similarity transformation was used to improve the numerical conditioning of state-space representation of $P$.

\subsubsection{Benchmark Controller}

Table 5.4 presents the weighting function parameters which were the result of several manual iterations to optimize the performance of the rotor-AMB system. The benchmark controller provided a base from which other controllers may be derived. By changing a single property at a time, we may develop a better understanding of the role of the weighting function parameters in determining the closed-loop performance. The resulting sensitivity bounds are shown in Figure 5.13. 
Table 5.4: Weighting function parameters for the benchmark controller.

\begin{tabular}{|c|c|c|}
\hline & NDE & DE \\
\hline \hline$M_{S}$ & \multicolumn{2}{|c|}{1.5} \\
\hline$M_{G S}$ & 0.13 & 0.08 \\
\hline$M_{K S}$ & $10 / M_{G S}$ & $12 / M_{G S}$ \\
\hline$\omega_{B}$ & \multicolumn{2}{|c|}{$130 \pi$} \\
\hline$\omega_{L}$ & \multicolumn{2}{|c|}{$8 \omega_{B}$} \\
\hline$K_{1}$ & \multicolumn{2}{|c|}{1.26} \\
\hline$K_{2}$ & \multicolumn{2}{|c|}{2.24} \\
\hline
\end{tabular}

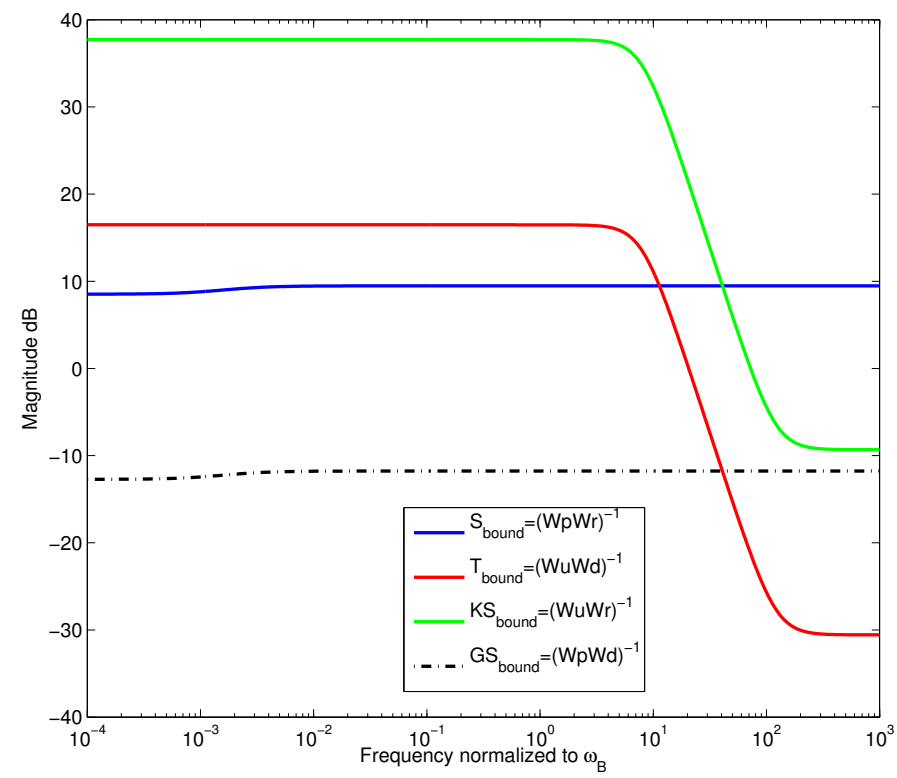

Figure 5.13: Sensitivity function bounds defined for the benchmark controller. 
Table 5.5: Weighting function parameters for family of controllers with support stiffness anisotropy $\chi$.

\begin{tabular}{|c|c|c|c|c|}
\hline & NDE-X & NDE-Y & DE-X & DE-Y \\
\hline \hline$M_{S}$ & \multicolumn{3}{|c|}{1.0} \\
\hline$M_{G S}$ & \multicolumn{2}{|c|}{0.13} & \multicolumn{2}{|c|}{0.08} \\
\hline$M_{K S}$ & $12 / M_{G S}$ & $12 \chi / M_{G S}$ & $15 / M_{G S}$ & $15 \chi / M_{G S}$ \\
\hline$\omega_{B}$ & \multicolumn{3}{|c|}{$130 \pi$} \\
\hline$\omega_{L}$ & \multicolumn{3}{|c|}{$8 \omega_{B}$} \\
\hline$K_{1}$ & \multicolumn{3}{|c}{1.26} \\
\hline$K_{2}$ & \multicolumn{3}{|c}{2.24} \\
\hline
\end{tabular}

\subsubsection{Introducing Support Anisotropy}

Support stiffness anisotropy, i.e., different AMB stiffness in the $x$ - and $y$-directions, was discussed in Chapter 3 as a strategy to increase the stability threshold. Anisotropy $\chi$ was introduced by weighting the control sensitivity function $K S(s)$ for one axis, e.g., $x$ more heavily to the other. Two different levels of stiffness anisotropy namely $\chi=50 \%$ and $\chi=$ $70 \%$ were studied. The stiffness in the $x$-direction remained the same as the benchmark case, whilst the stiffness in the $y$-direction was scaled by $\chi$. The new weighting function parameters are indicated in Table 5.5. The change in $M_{K S}$ only affects the weight on the controller output $W_{u}$. All the other weighting functions are identical to the benchmark case.

\subsubsection{Introducing Nondiagonal Performance Weights}

Nondiagonal performance weights have been discussed and show promise as means of maximally exploiting the available degrees of freedom during control synthesis (Boerlage, 2008). The MBTRI plant is largely diagonally dominant at low frequencies in the absence of significant cross-coupling. Enforcing a similar diagonal dominance in the control algorithm is akin to synthesizing a centralized controller. In the presence of significant crosscoupling the plant becomes less diagonally dominant at these frequencies. This suggests that a controller synthesized with nondiagonal weighting functions may have additional degrees of freedom to optimize the overall closed-loop sensitivity leading to performance 
improvements. Finding a suitable form of the performance weight matrix is complicated given the large parameter search space. Therefore, a heuristic approach was followed to narrow the search. The first step was the development of four prototype nondiagonal scalings to systematically evaluate the effects of coupling and directionality between multiple control channels:

1. Full block performance weight

$$
W_{p, 1}(s)=\left[\begin{array}{cccc}
1 & 1 & 1 & 1 \\
1 & 1 & 1 & 1 \\
1 & 1 & 1 & 1 \\
1 & 1 & 1 & 1
\end{array}\right] \text { blkdiag }\left[W_{p, N D E}, W_{p, D E}, W_{p, N D E}, W_{p, D E}\right]
$$

2. Cross-coupled performance weight

$$
W_{p, 2}(s)=\left[\begin{array}{cccc}
1 & 0 & 0.5 & 0 \\
0 & 1 & 0 & 0.5 \\
0.5 & 0 & 1 & 0 \\
0 & 0.5 & 0 & 1
\end{array}\right] \text { blkdiag }\left[W_{p, N D E}, W_{p, D E}, W_{p, N D E}, W_{p, D E}\right]
$$

3. Simple block-diagonal

$$
W_{p, 3}(s)=\left[\begin{array}{cccc}
1 & 1 & 0 & 0 \\
1 & 1 & 0 & 0 \\
0 & 0 & 1 & 1 \\
0 & 0 & 1 & 1
\end{array}\right] \text { blkdiag }\left[W_{p, N D E}, W_{p, D E}, W_{p, N D E}, W_{p, D E}\right]
$$

4. Block-diagonal with scaled off-diagonal terms 


$$
W_{p, 4}(s)=\left[\begin{array}{cccc}
1 & 0.5 & 0 & 0 \\
0.5 & 1 & 0 & 0 \\
0 & 0 & 1 & 0.5 \\
0 & 0 & 0.5 & 1
\end{array}\right] \text { blkdiag }\left[W_{p, N D E}, W_{p, D E}, W_{p, N D E}, W_{p, D E}\right]
$$

5. Block-diagonal with negative scaled off-diagonal terms

$$
W_{p, 5}(s)=\left[\begin{array}{cccc}
1 & -0.5 & 0 & 0 \\
-0.5 & 1 & 0 & 0 \\
0 & 0 & 1 & -0.5 \\
0 & 0 & -0.5 & 1
\end{array}\right] \text { blkdiag }\left[W_{p, N D E}, W_{p, D E}, W_{p, N D E}, W_{p, D E}\right]
$$

The five controllers synthesized kept the same parameters for $W_{u}, W_{d}$ and $W_{r}$ as the benchmark case.

\subsection{Synthesis Results}

A total of seventeen different controllers were synthesized using a combination of the weighting functions and uncertainty models presented. In all cases, the D-K iteration procedure converged within 5 steps to a stabilizing controller of order ranging from 48 and 56. The iteration sequence for the benchmark controller is presented in Table 5.8 and $\mu$-upper bound is shown in Figure 5.14. A result of $\mu_{\Delta}(M) \approx 1$ is desired, for it reflects that the closed-loop performance defined by the weighting functions can be achieved for all plant perturbations in the defined uncertainty set. A result of $\mu_{\Delta}(M)<1$ indicates that controller can either guarantee performance exceeding the definition, or a superset of the uncertainty description or both. Therefore, the actual $\mu$ value is only of relative performance, as it's reciprocal specifies the extent the performance specification and uncertainty set may be scaled to meet the RP objective. Identifying the individual contributions of stability, performance and uncertainty to the overall value of $\mu$ is performed in the next section on 
analysis.

The details on controllers synthesized using either Speed Model 1 or Speed Model 2 for supercritical operation are summarized in Table 5.6. Table 5.7 summarizes the details on controllers synthesized with both Speed Model 2 and one of the four CCS uncertainty models. Almost all of the synthesized controllers were able to levitate rotor-AMB system. Controllers that failed to do so employed either Speed Model 1, or CCS Models 1 or 2. In the case of Speed Model 1 the default D-K iteration initially did not converge due to numerical issues. However, by manually specifying the frequency grid over a smaller band, the D-K iteration was able to proceed through two iterations before halting with a 206 state controller (Kdk_206_17Aug11z). The size of this controller was reduced to 60 states prior to discretization (using a reduction based on the Hankel norm approximation) to enable execution within the fixed sample time of DSP. However, the rotor could not be suspended stably by this control law. This result was indicative of the conservatism of the direct modeling approach used to capture the variation in rotating speed as an uncertain parameter. As a result Speed Model 2 is used exclusively to represent the speed uncertainty. The closed-loop instability in the case of CCS Models 1 and 2 was unsurprising as the unity gap metric predicted significant differences between the closed-loop behavior of the nominal plants based upon these CCS uncertainty descriptions when compared to the nominal plant model without CCS.

\subsubsection{Evaluation of the Benchmark Controller}

As mentioned previously, the benchmark controller (Kdk_48_17Aug11a) was developed after several iterations of the weighting function modification and control synthesis. The benchmark serves as the root from which other controllers were developed. Key properties of the benchmark controller are evaluated below to highlight the details of its construction. The analysis largely focuses on the control response, and the Bode plots and the singular value plots are used to show the magnitude and phase, MIMO response, and closed-loop 


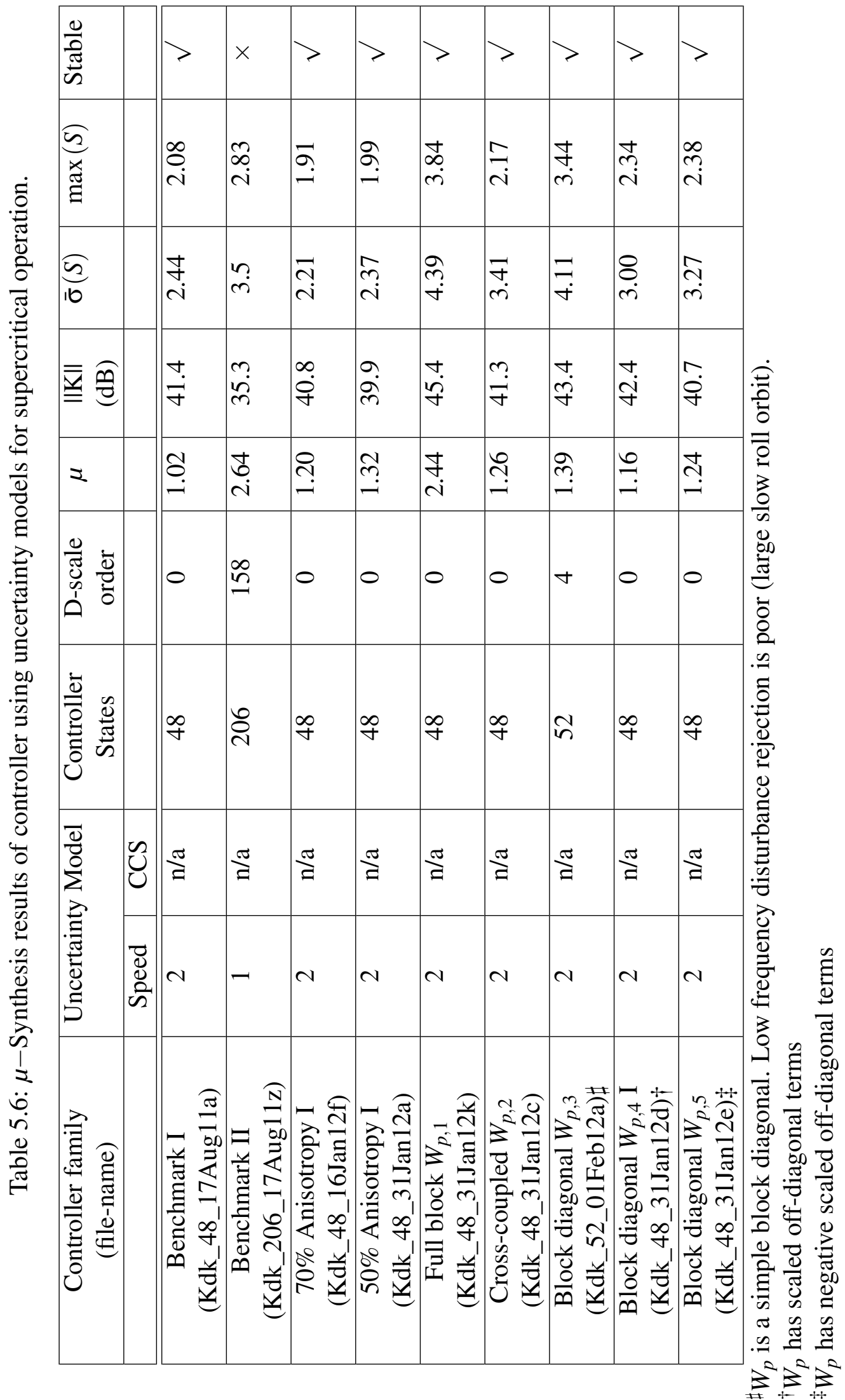




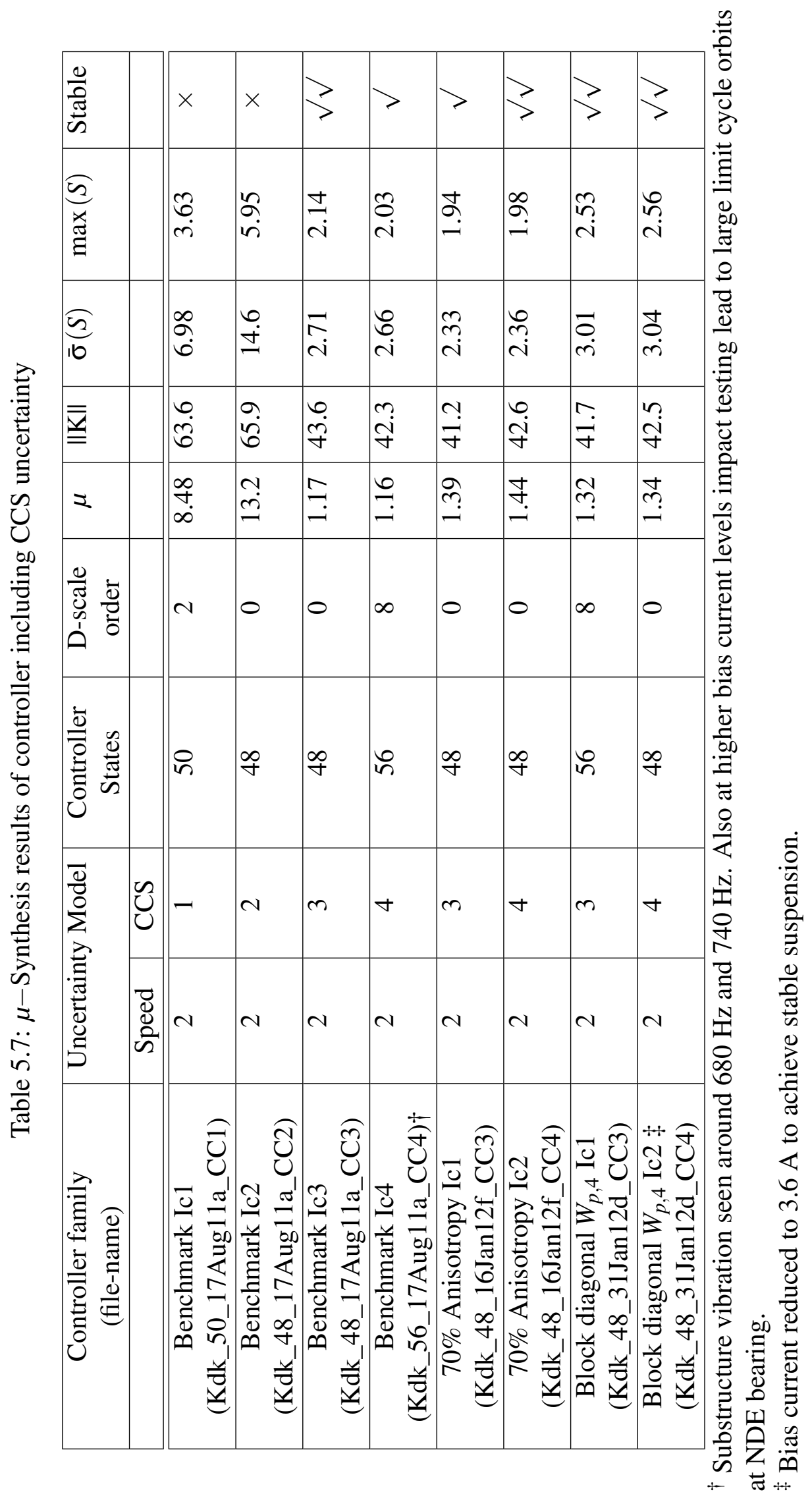


actuator stiffness of the controller.

The D-K iteration steps during the synthesis of the benchmark controller are tabulated in Table 5.8. Convergence of the $\mu$-upper bound occurred within 4 iterations. A zeroth order D-scaling matrix was generated during each iteration. This results in the minimum achievable controller order of 48. A discrete-time implementation of this control law sampled at $12 \mathrm{kHz}$ executes within $52 \mu$ s on the DSP hardware. This represents $62 \%$ of the available interrupt (Mushi et al., 2011). The evolution of the $\mu$-upper bound as a function of frequency during the D-K iterations is shown in Figure 5.14 and the minimum peak value of $\mu$ is 1.02 .

Bode plots for the magnitude and phase response along all the controller channels are shown in Figure 5.16. A total of 16 transfer functions represent the dynamics from the four sensor inputs to the four amplifier inputs. The diagonal gains, i.e., $\mathrm{X} \rightarrow \mathrm{X}$ and $\mathrm{X} \rightarrow \mathrm{Y}$, dominate the controller response. The phase behavior of the controller reveals that the rigid body modes ( $\mathrm{Nc} 1$ and $\mathrm{Nc} 2$ ) and the first bending mode (Nc3) are well damped by the positive phase lead in their vicinity. The second bending mode Nc4 is gain stabilized by notch filters, while the third bending mode is gain stabilized by the controller gain roll-off. The singular value plot (Figure 5.15) generalizes the multivariable response and is shown together with the singular value plot of the nominal plant augmented with Speed Model 2.

Analyzing the pole and zero locations of the controller is important to examine whether pole-zero cancellation has occurred between the controller and plant. Pole-zero cancellation is at best highly undesirable due to the absence of robust stabilization, and at worst likely to produce unstable controllers if the plant has RHP zeros. The poles and zeros of the benchmark controller are shown in Figure 5.17, where it is confirmed no cancellation with plant poles or zeros occurs. This is a byproduct of using the four-block mixed sensitivity framework for specifying the controller performance. Interestingly, the controller contained four pairs of complex RHP zeros at $205 \pm j 3449,189 \pm j 3431,81 \pm j 1180$, and $80 \pm j 1175$. These non minimum-phase zeros introduce phase lag in the vicinity of the 
Table 5.8: D-K iteration results for Benchmark I controller (Kdk_48_17Aug11a).

\begin{tabular}{|c|c|c|c|c|c|}
\hline & 1 & 2 & 3 & $4^{*}$ & 5 \\
\hline \hline States & 48 & 48 & 48 & 48 & 48 \\
\hline$D$-scale order & 0 & 0 & 0 & 0 & 0 \\
\hline$\gamma$ & 1.879 & 1.039 & 1.030 & 1.02 & 1.02 \\
\hline$\mu$ & 1.45 & 1.04 & 1.03 & 1.02 & 1.02 \\
\hline
\end{tabular}

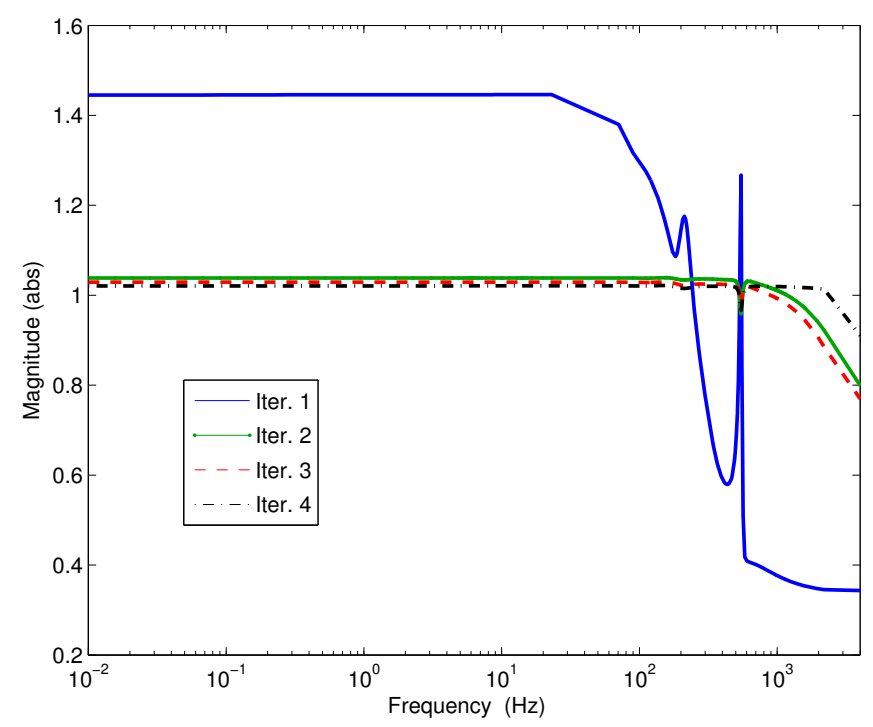

Figure 5.14: Changes in $\mu$ during D-K iteration steps for the Benchmark I controller.

bending modes and serve to augment their damping (Li, 2006).

The equivalent closed-loop mechanical stiffness of AMB actuator as a function of frequency (Williams et al., 1990) is

$$
K_{\mathrm{eq}_{i j}}(j \omega)=K_{i} G_{s} G_{\mathrm{as}} \operatorname{Re}\left(K_{i j}(j \omega)\right)
$$

where $G_{S}$ is the sensor gain, $G_{\text {as }}$ is the amplifier gain, $K_{i}$ is the AMB current gain and $K_{i j}(j \omega)$ is the frequency response of the $i, j$ controller channel. This equivalent stiffness is plotted in Figure 5.18 can be compared to similar plots for mechanical bearings. The plots show that a similar stiffness is apparent along the $x$ and $y$ axes of each bearing, while the driven-end bearing has an overall higher low-frequency stiffness than the non driven-end bearing. 


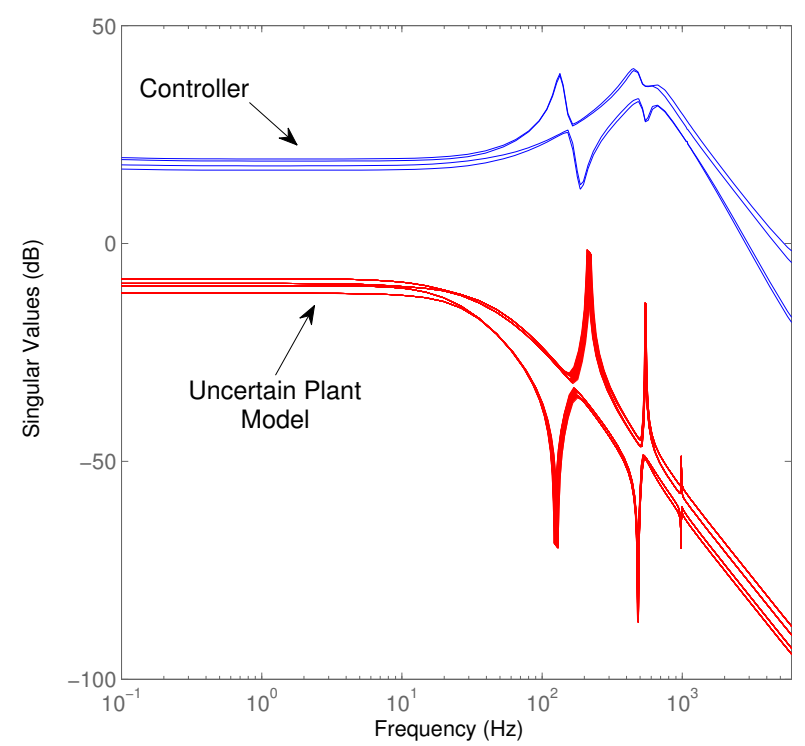

Figure 5.15: Singular value of Benchmark I controller and plant with Speed Model 2. 


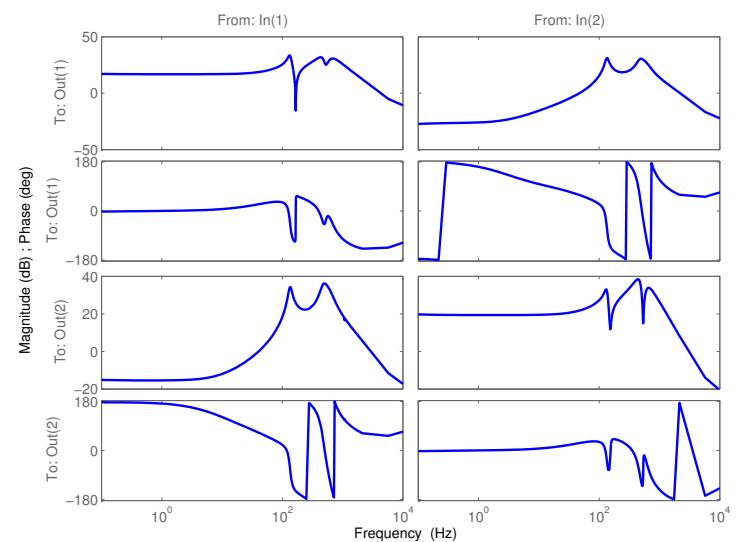

(a) $\mathrm{X} \rightarrow \mathrm{X}$

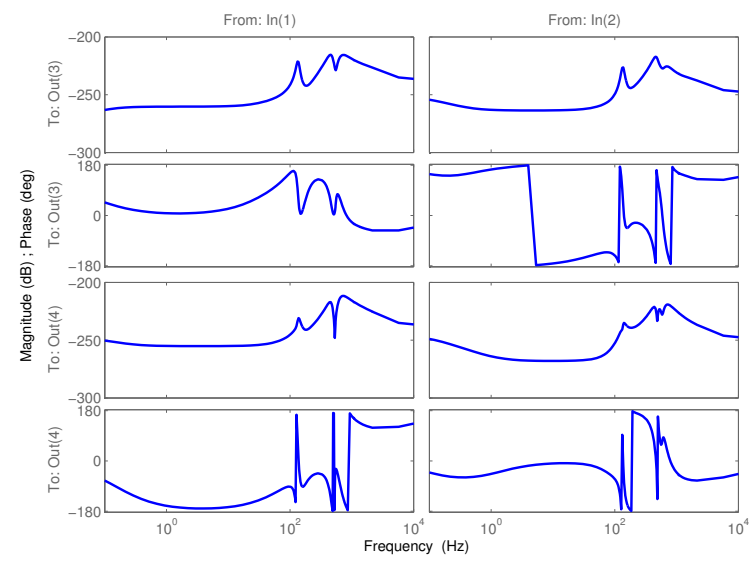

(c) $\mathrm{X} \rightarrow \mathrm{Y}$

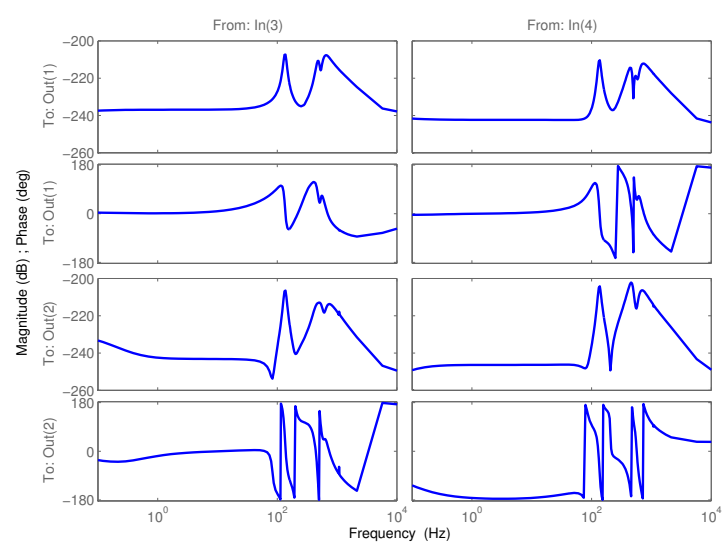

(b) $\mathrm{Y} \rightarrow \mathrm{X}$

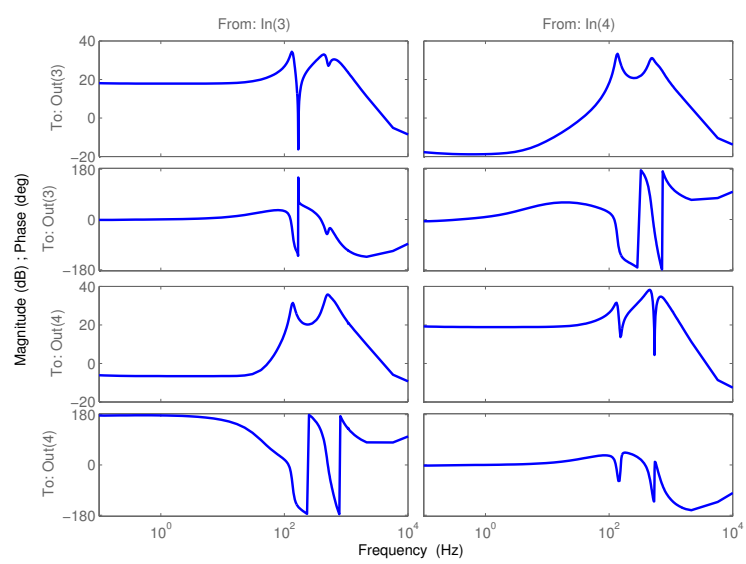

(d) $\mathrm{Y} \rightarrow \mathrm{Y}$

Figure 5.16: Bode plots of response of Benchmark I controller across all four channels. In and Out refer to input and output directions, while the indices 1, 2, 3 and 4 refer to NDE-X, DE-X, NDE_Y and DE-Y, respectively. 


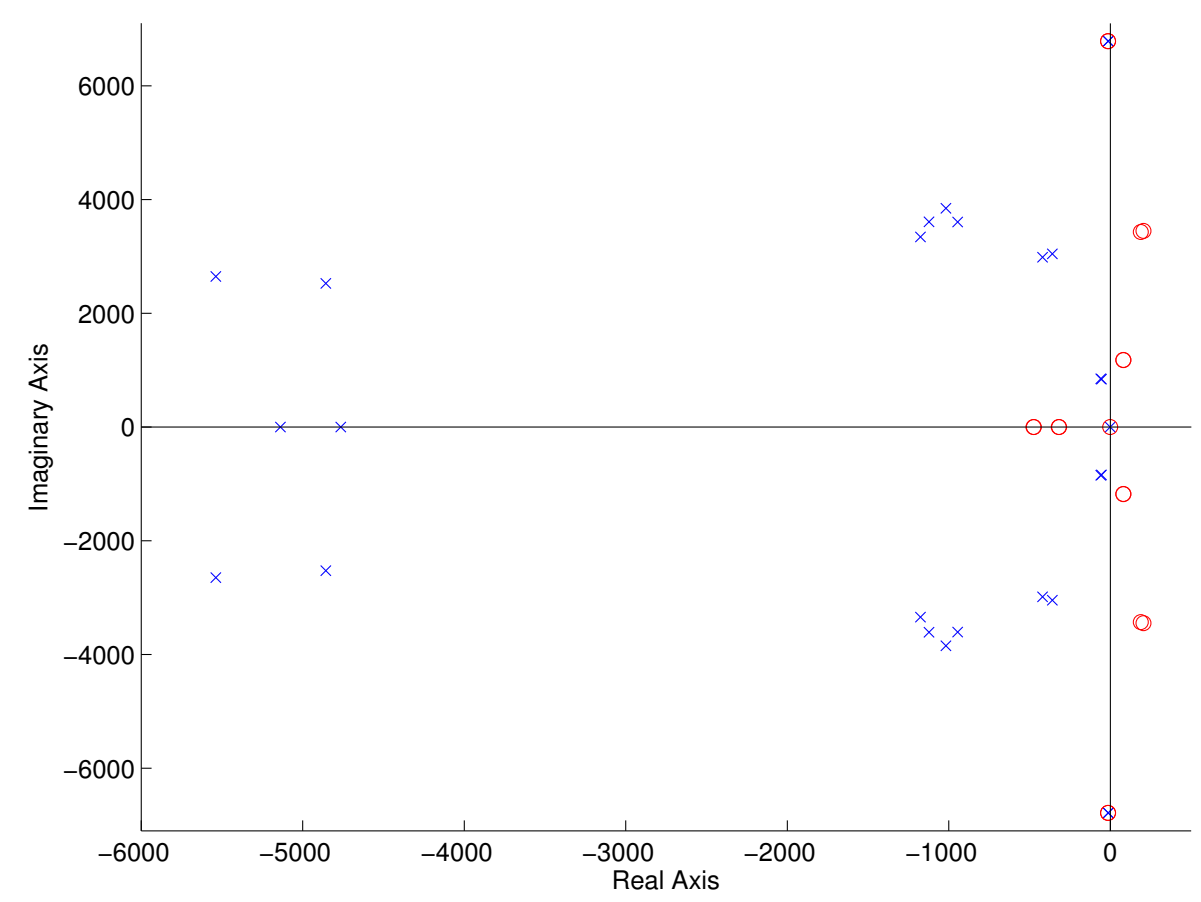

Figure 5.17: Pole-zero map of the Benchmark I controller (excludes 2 real poles at -242 $\mathrm{krad} / \mathrm{s}$ and $-56 \mathrm{krad} / \mathrm{s}$ and a complex zero pair at $34.6+j 34.7 \mathrm{krad} / \mathrm{s})$.
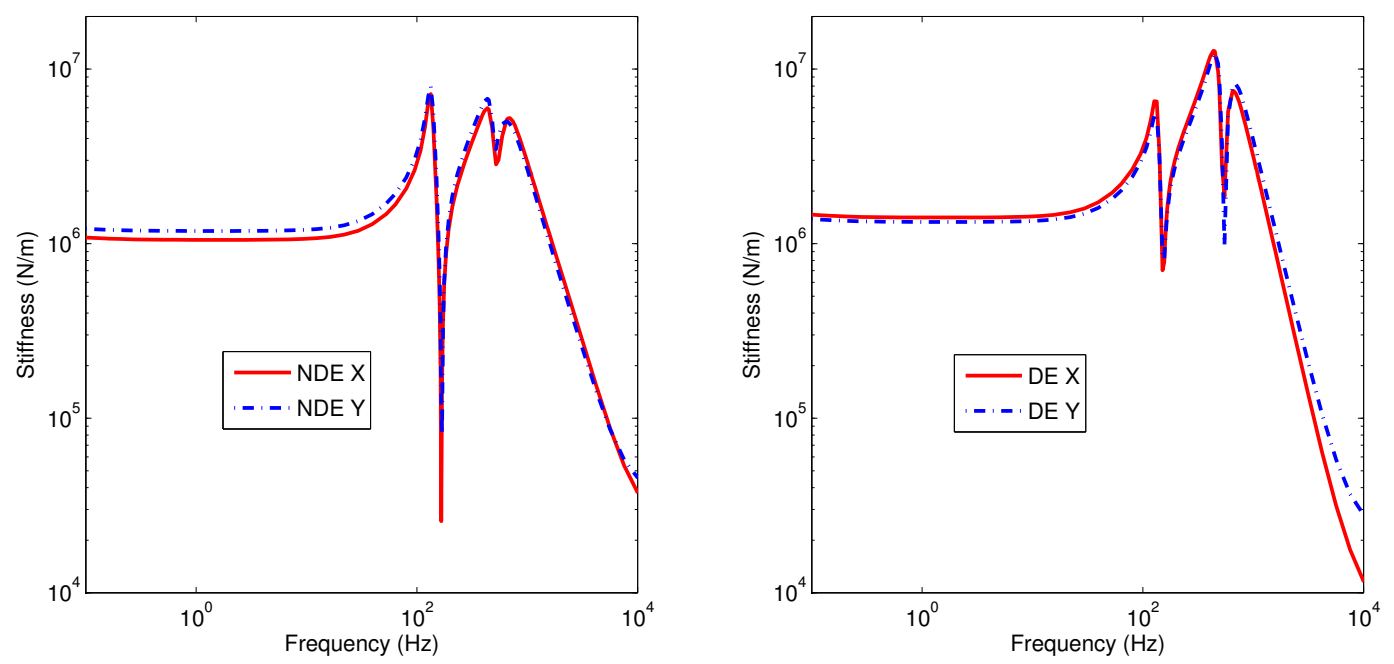

Figure 5.18: Predicted local closed-loop actuator stiffness of Benchmark I controller. 


\section{$5.6 \mu$-Analysis}

Recapping, the objective of $\mu$-synthesis is to find the controller $K$ which minimizes the structured singular value of the generalized plant $M$ with respect to the uncertainty definition $\Delta$ within the $\Delta$. If $\mu_{\Delta}(M) \leq 1 \forall \omega$, we can say with confidence that $K$ meets or exceeds the performance specification for worst case perturbation in $\Delta$. What can we say if $\mu_{\Delta}(M)>1$ ? Superficially, it is obvious that either the performance specifications are too stringent, or the uncertainty set is too broad. $\mu$-Analysis provides the mechanics to determine where the uncertainty or performance specifications are the limiting factors in the overall closed-loop behavior. Using LFTs, the nominal plant, performance specifications, and controller are combined into a $2 \times 2$ block transfer matrix $M$ (see Equation (5.4) and Figure 5.19). By performing analyses on components of $M$, we can separate the contributions of the nominal performance (NP) and robust stability (RS) specifications from the robust performance specification (RP). The maximum singular value of $M_{11}$, i.e., $\bar{\sigma}\left(M_{11}\right)$, is the system gain from the uncertainty description inputs $v$ to the uncertainty description outputs evaluated over a given frequency range and describes worst case gain interaction between the plant dynamics interaction uncertainty description. RS is guaranteed if $\bar{\sigma}\left(M_{11}\right)<1$. Similarly, $\bar{\sigma}\left(M_{22}\right)$ is the system gain from $w$ to the performance metric $z$ which consists of the weighted position errors $\left(z_{1}=W_{p} e\right)$ and the weighted controller output $\left(z_{2}=W_{u} u\right)$. NP is guaranteed if $\bar{\sigma}\left(M_{22}\right)$. Further, the transfer matrix $M_{22}$ may be partitioned to analyze the relative contribution of disturbance inputs $d$ and reference input signal $r$ to both the weighted error $z_{1}$ and the weighted control signal $z_{2}$ (Honeywell \& Lockheed-Martin, 1996). The solution to the RP is found by evaluating the $\mu$-upper bound $\mu_{\Delta}(M(j \omega))$ at distinct frequencies over a specified range. How close $\bar{\sigma}\left(M_{11}\right)$ and $\bar{\sigma}\left(M_{22}\right)$ are to $\mu_{\Delta}(M(j \omega))$ reveal the relative contributions of the composition of the uncertainty set and performance specifications to the overall $\mu$ upper bound. Balancing the controller's emphasis between nominal performance specifications and robust stability specs is very important from an engineering viewpoint (Bates \& Postlethwaite, 2002). In the following 


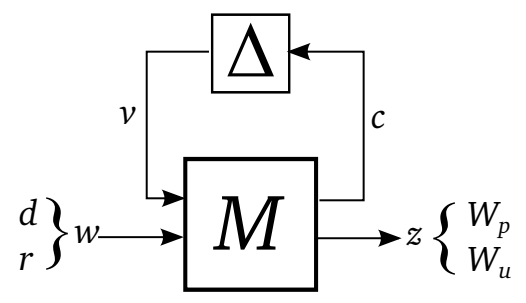

Figure 5.19: $M-\Delta$ analysis

sections controllers designed (see Tables 5.6 and 5.7) with various combinations of performance weighting functions and plant model uncertainty descriptions are compared to the benchmark controller to examine the nature of the trade offs made during the optimization process and reveal possible directions for improvement. The approach taken during the analysis, is similar to the paradigm followed in the synthesis section, i.e., stepwise modification a individual aspects of either the performance weight, or uncertainty model to reveal their relative importance and contribution to the overall control objectives.

\subsubsection{Controllers with Speed Uncertainty}

The two plant uncertainty models examined in this section are Speed Model 1 and Speed Model 2. They capture the changes in the plant dynamics as the operating speed is varied from 0 to $18,000 \mathrm{rpm}$. In the previous section it was noted that the benchmark controller designed with the Speed Model 2 surpassed the stability and performance of the same controller structure designed with Speed Model 1. This is because the latter was unable to levitate the rotor at $0 \mathrm{rpm}$. The reasons are pretty clear by comparing Figures 5.20 and 5.21. The RS bound in both Figure 5.20a and 5.21a are less than 0.4 for low frequencies suggesting that the uncertainty set could be expanded by $250 \%$ without sacrificing stability. However, as Speed Model 1 contains the gyroscopic effect of Nc5 (which has the largest split of all eigenvalues, see Figure 4.9) around $900 \mathrm{~Hz}$, the RS bound rises above unity. Furthermore, the nominal and robust performance of the controller designed with Speed Model 1 deteriorates significantly below $100 \mathrm{~Hz}$. This is due to the coupling introduced between the $x$ and $y$ axes by the full gyroscopic model. Speed Model 2 does not exhibit this 


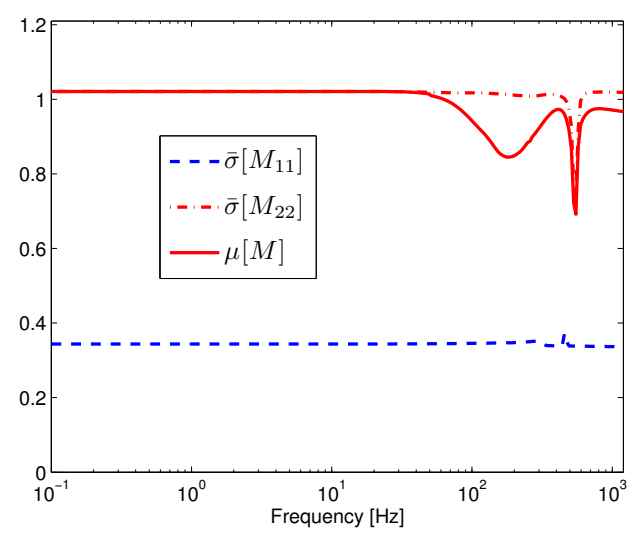

(a) NS, NP, RP

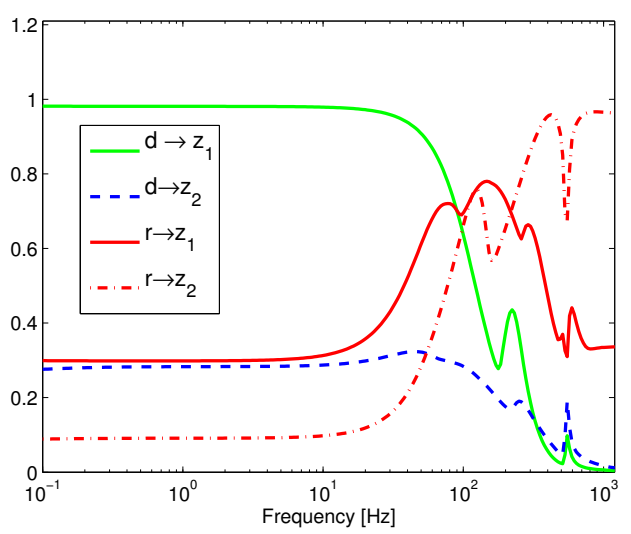

(b) Nominal performance breakdown

Figure 5.20: $\mu$-Analysis of Benchmark I controller designed with plant uncertainty including Speed Model 2.

coupling, and there has a NP and RP bound of at most 1.02 for the same frequencies. The major contribution to the NP bound in both systems (and hence RP since they are close) is the effect of disturbance inputs on the weighted position error, i.e., $d \rightarrow z_{1}$, at frequencies below $100 \mathrm{~Hz}$ (see Figure 5.20b and Figure 5.21b). This suggests that altering the weight $W_{p}$ will have the biggest affect on performance. At higher frequencies, the gain from $r \rightarrow z_{2}$ dominates to ensure the controller gain roll-off at high frequencies.

The next comparison examined is between the result of Figure 5.20 and two controllers with different performance weighting functions: Kdk_48_16Jan12f and Kdk_48_31Jan12d. The former incorporates 70\% support stiffness anisotropy, and the latter features a block diagonal $W_{p}$ with scaled off-diagonal terms. The $\mu$-upper bound increases from 1.02 for the benchmark to 1.20 and 1.16 for Kdk_48_16Jan12f and Kdk_48_31Jan12d, respectively as shown in Figures 5.22a and 5.23a. The RP bound of 0.35 is relatively unchanged among the three controllers which is expected as they are derived from plants with the same uncertainty description, i.e., Speed Model 2. While the NP and RP of these controllers are inferior to the benchmark at frequencies below about $100 \mathrm{~Hz}$, in the frequency range surrounding the Nc3 $(200 \mathrm{~Hz}) \mathrm{Kdk} \_48 \_31 \mathrm{Jan} 12 \mathrm{~d}$ demonstrates a $\mu$-bound of nearly 0.6 versus approximately 0.8 for the benchmark controller. The use of block diagonal performance 


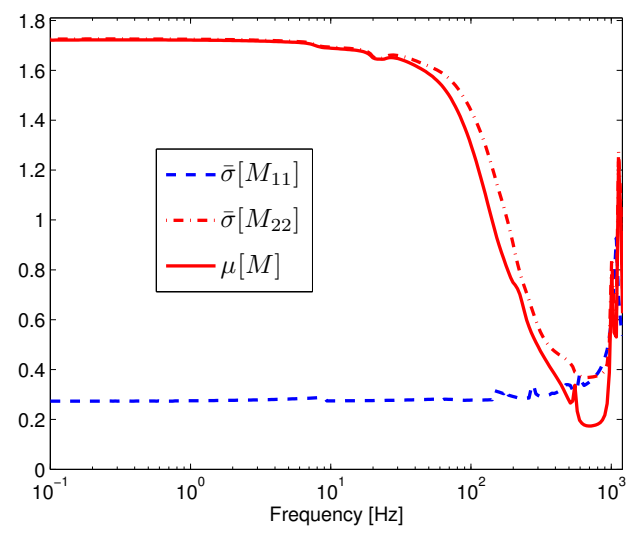

(a) NS, NP, RP bounds.

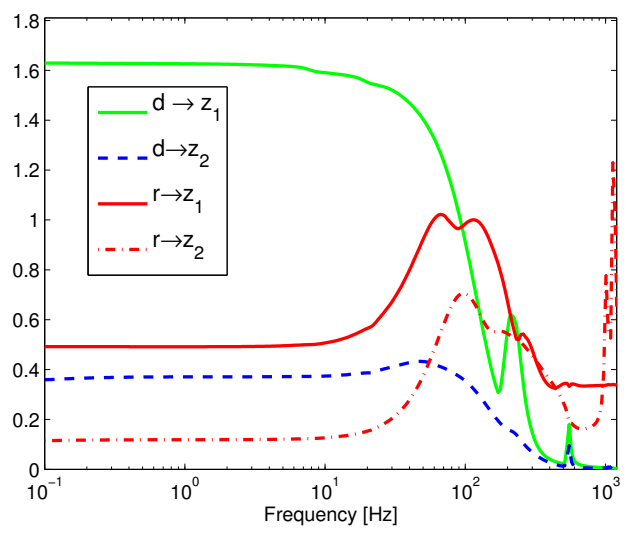

(b) Nominal performance breakdown

Figure 5.21: $\mu$-Analysis of benchmark controller designed with plant uncertainty including Speed Model 1.

weights provides the most significant improvement to the closed-loop disturbance rejection and reference tracking around the bending modes. This is confirmed in Figure 5.23b where the $d \rightarrow z_{1}$ and $r \rightarrow z_{1}$ plots reach lower than Figure 5.20b. A small penalty does remain in the form of reduced performance below $100 \mathrm{~Hz}$. However, as we shall see in the Section 6.1.3, both of the new controllers demonstrate a higher stability threshold with respect to CCS than the benchmark.

\subsubsection{Controllers with CCS Uncertainty}

The aim of this section is to examine the changes introduced by the addition of a CCS uncertainty description to a plant model already augmented with the Speed Model 2 uncertainty. Four different CCS uncertainties are considered in this section, i.e., CCS Models 1 through 4 .

First we compare the $\mu$-bounds of closed-loop systems stabilized by Benchmark controller I to those stabilized by Benchmark Ic1 (Figure 5.24). As we know from the synthesis results, Benchmark Ic1 (and Ic2) have $\mu \gg 1$ and are unable to achieve stable suspension of the rotor at $0 \mathrm{rpm}$. This is particularly evident by the peaks above unity for the RS plot in Figure 5.24a. These peaks are indicative of sensitivity of the system stability to 


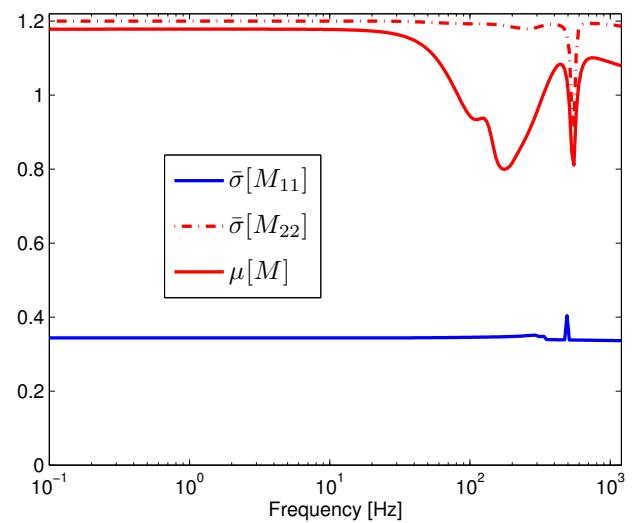

(a) NS, NP, RP bounds.

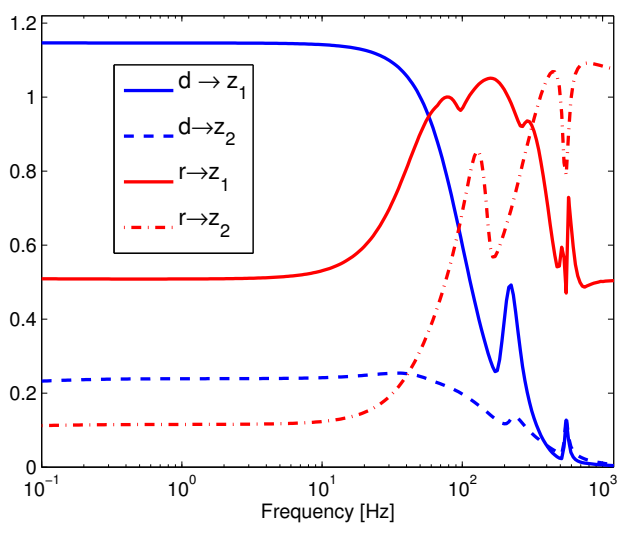

(b) Nominal performance breakdown

Figure 5.22: $\mu$-Analysis of $70 \%$ stiffness anisotropy designed with plant uncertainty including Speed Model 2.

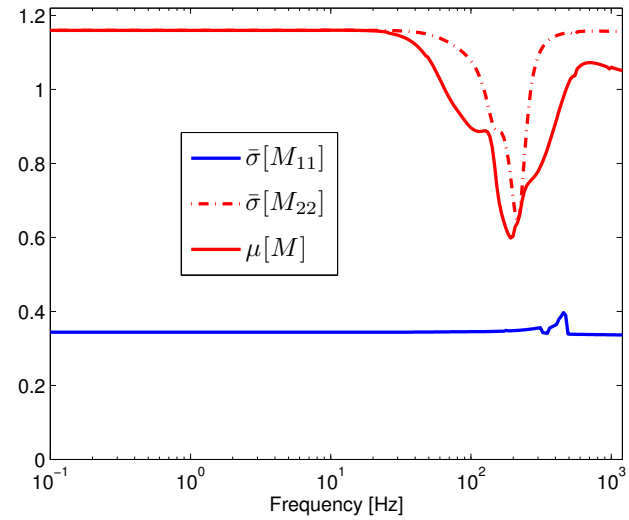

(a) NS, NP, RP bounds.

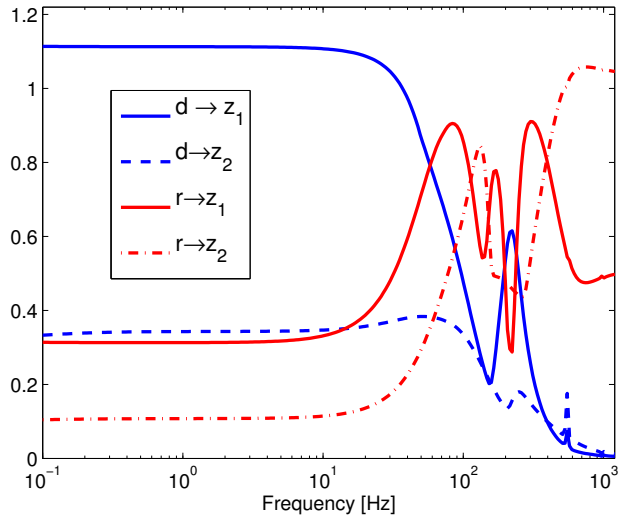

(b) Nominal performance breakdown

Figure 5.23: $\mu$-Analysis of nondiagonal performance weight $W_{p, 4}$ controller designed with plant uncertainty including Speed Model 2. 
small changes in the eigenvalue locations. Analysis of details of the NP in Figure 5.24b reveals that the system gain below $100 \mathrm{~Hz}$ from disturbance inputs to weighted position error $\left(d \rightarrow z_{1}\right)$ is nearly three times the value achieved with Benchmark I. Similarly, the system gain from reference input to weighted control output $\left(r \rightarrow z_{2}\right)$ has a value close to eight times the equivalent Benchmark I value at high frequencies. These results show that direct uncertainty modeling approach used in CCS Model 1 (and CCS Model 2) provides nominal plants and uncertainty descriptions that are not ideal for controller synthesis.

The $\mu$-bounds of Benchmark Ic3 (Figure 5.25) and Ic4 (Figure 5.26) are now compared to those of Benchmark I. For the most part the RS is unchanged by including CCS Model 3 or 4 into the synthesis, with the maximum value of $\bar{\sigma}\left(M_{11}\right)<0.4$ across the entire frequency range. The low frequency NP and RP bounds increase slightly to 1.16 and 1.17 , respectively, for the new controllers. This small reduction in performance was expected since the unstable $\mathrm{Nc1}$ eigenvalue has been moved deeper into the right half plane which impacts the allowable sensitivity reduction. The nominal performance breakdown in Figures 5.25b and 5.26b reveals very similar nominal performance to Benchmark I except the disturbance rejection at low frequencies which was a small penalty. Nominal performances similar to the Benchmark I are desirable, as they generally indicate that stable suspension of rotor by the candidate controller can be achieved at $0 \mathrm{rpm}$ with no external excitation, i.e., under nominal conditions.

The two controllers synthesized with block-diagonal performance weight $W_{p, 4}$ and either CCS Model 3 or CCS Model 4 yielded a smaller increase in $\mu$ from Benchmark Ic3 and Ic4 as compared to the controller designed with $70 \%$ support stiffness anisotropy. Therefore, the RS, NS and NP problems will be examined in detail for these two controllers. As shown in Figures 5.27a and 5.28b, the RS bounds are not very different from the Benchmark Ic3 case, except for a sharp peak close to $200 \mathrm{~Hz}$ seen with CCS Model 3. Breaking down the nominal performance of these two controllers again reveals similar system gain behavior with frequency to Benchmark Ic3. As was noted in Table 5.7, a lower bias current 


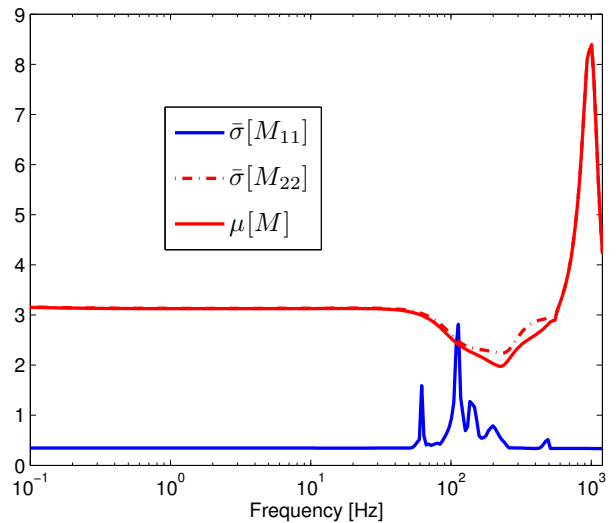

(a) NS, NP, RP bounds.

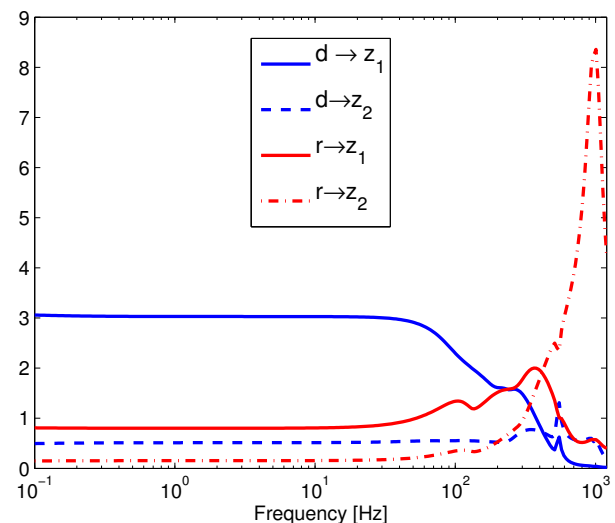

(b) Nominal performance breakdown

Figure 5.24: $\mu$-Analysis of Benchmark Ic1 controller designed with plant uncertainty including Speed Model 2 and CCS Model 1.

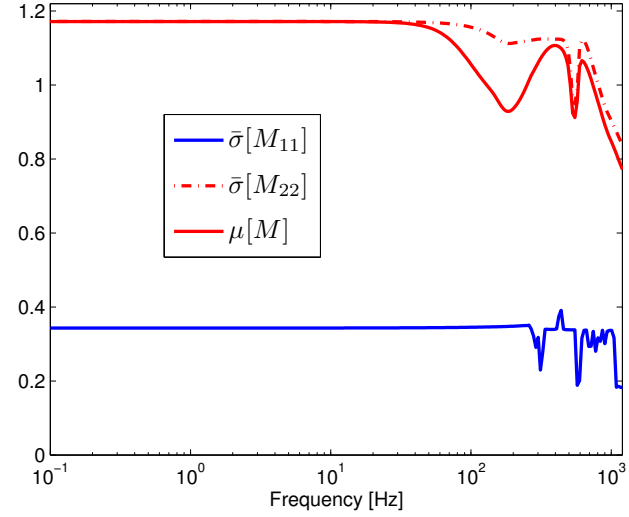

(a) NS, NP, RP bounds.

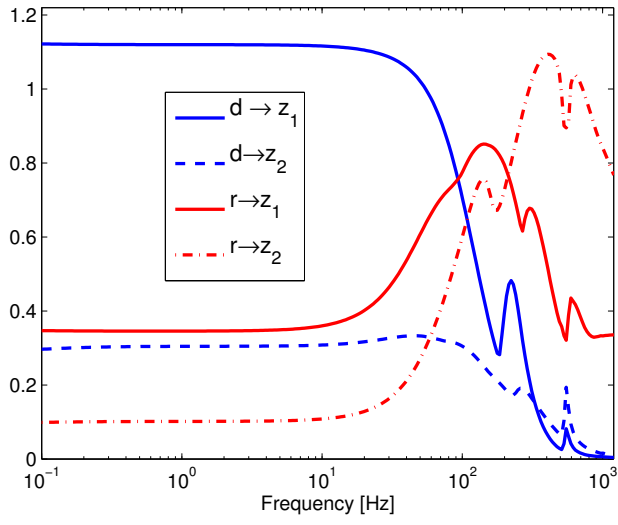

(b) Nominal performance breakdown

Figure 5.25: $\mu$-Analysis of Benchmark Ic3 controller designed with plant uncertainty including Speed Model 2 and CCS Model 3. 


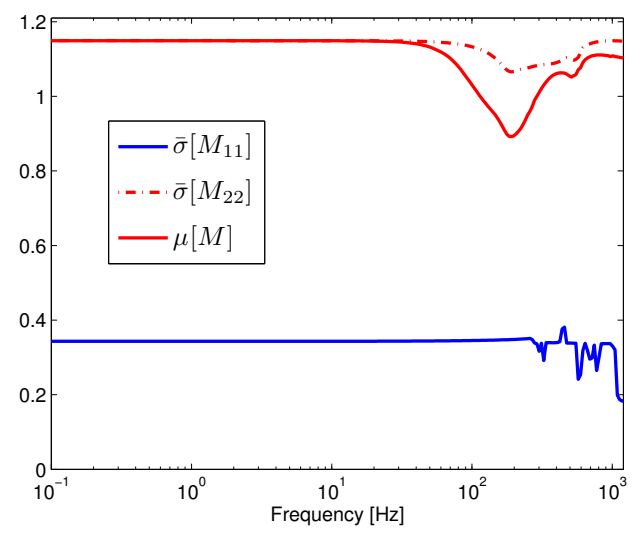

(a) NS, NP, RP bounds.

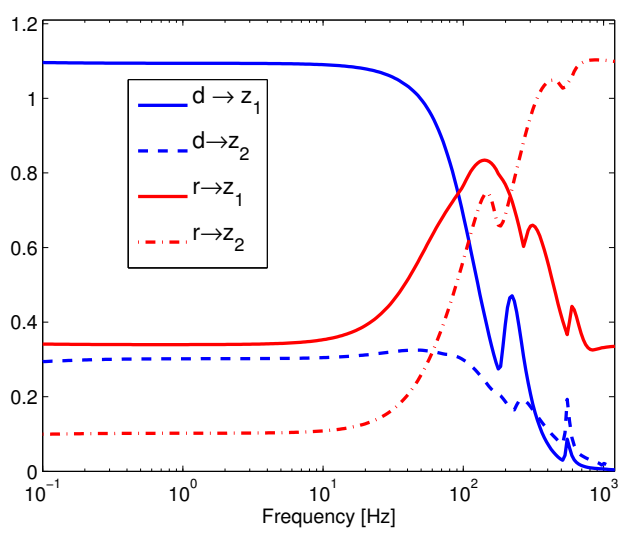

(b) Nominal performance breakdown

Figure 5.26: $\mu$-Analysis of Benchmark Ic4 controller designed with plant uncertainty including Speed Model 2 and CCS Model 4.

was necessary for block diagonal $W_{p, 4}$ Ic 2 controller to achieve stable suspension of the rotor. This is likely to contribute to slightly reduced performance with respect to CCS since a higher bias current is associated with higher values of the AMB current $K_{i}$.

\subsection{Rotordynamic Analysis}

As mentioned in Section 4.2.1.1, the damped rotordynamic analyses provide more complete picture of the rotor behavior by including the effects of support damping. However, such analysis may only be performed once a suitable AMB controller has been designed. In this section we evaluate the forced response and rotordynamic stability of the Benchmark I controller. The forced response to unbalance and the stability (damped mode shape) analyses are computations used to predict the likely performance of the rotor system over its operating speed range prior to experimental test. The seventh edition of the American Petroleum Industry Standard 617 concerning centrifugal compressors in petroleum, chemical or gas industry service is the most relevant standard for rotordynamic analysis, and provides a context for our analysis (API 617, 2002). 


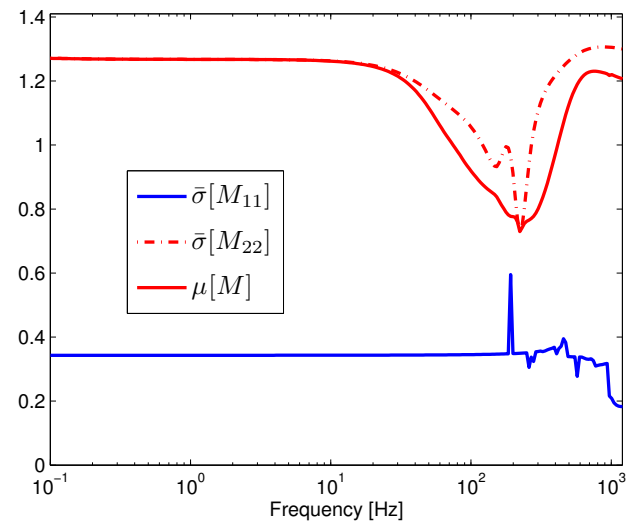

(a) NS, NP, RP bounds.

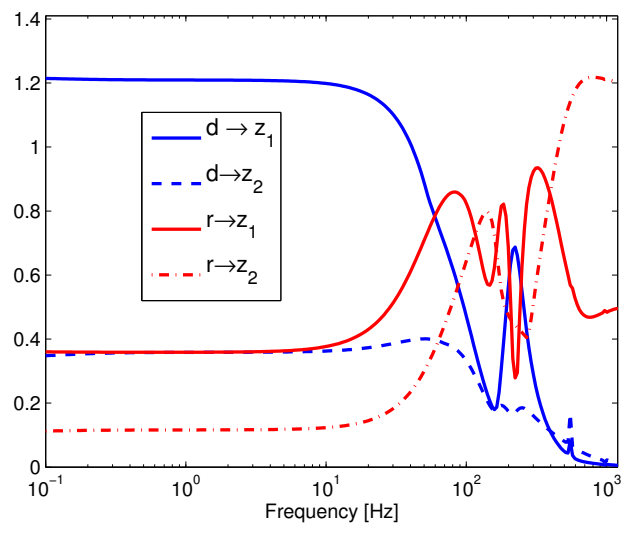

(b) Nominal performance breakdown

Figure 5.27: $\mu$-Analysis of nondiagonal performance weight $W_{p, 4}$ controller designed with plant uncertainty including Speed Model 2 and CCS Model 3.

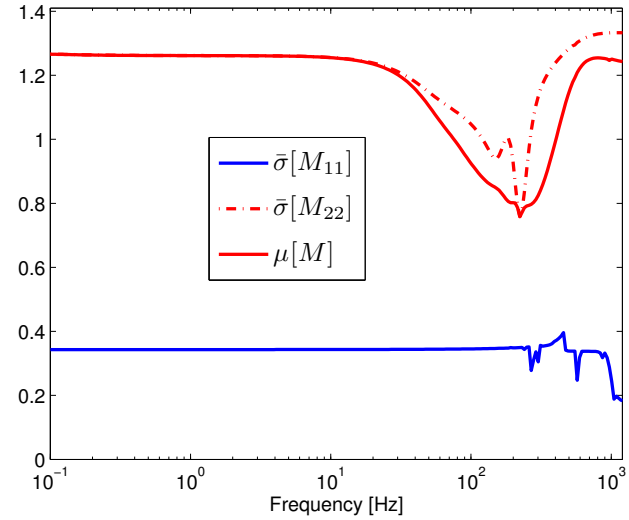

(a) NS, NP, RP bounds.

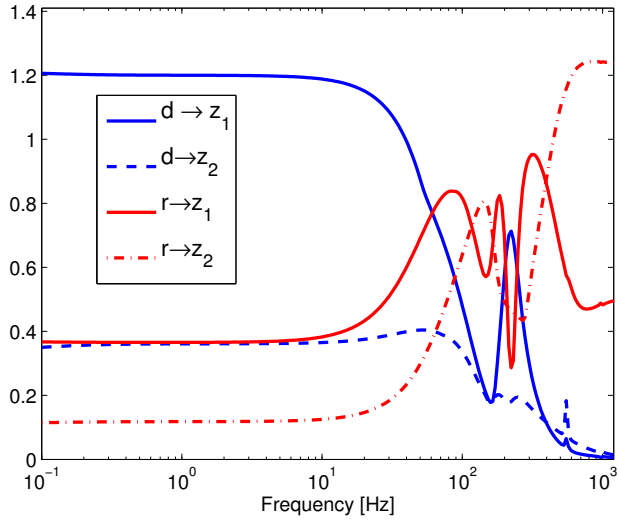

(b) Nominal performance breakdown

Figure 5.28: $\mu$-Analysis of nondiagonal performance weight $W_{p, 4}$ controller designed with plant uncertainty including Speed Model 2 and CCS Model 4. 


\subsubsection{Forced Response to Unbalance}

The objective of this simulation is to record the vibration response of the rotor over its operating speed range under various worst case imbalance conditions. The imbalance is emulated by adding known masses to different locations along the rotor. The resulting residual unbalance distributions excite different rotor modes during the run-up, and the objective is to maintain a satisfactory vibration amplitude and Q-factor (amplification factor during resonance) within the API guidelines. According to the API guidelines the unit unbalance eccentricity $u_{B}$ added to the rotor is

$$
u_{B}=6350 \mathrm{~W} / \mathrm{N}=15.9 \mathrm{~g}-\mathrm{mm}
$$

where the rotor mass $W=45 \mathrm{~kg}$ and maximum running speed $N=18,000 \mathrm{rpm}$. To simulate the worst case response the unbalance eccentricity is scaled by four to produce a unit unbalance eccentricity of $4 u_{B}$ which is placed at different axial and relative angular displacements in order to excite specific rotor modes. Two unbalance configurations are considered:

Case 1 place $4 u_{B}$ at NDE AMB location and $4 u_{B}$ at DE AMB location, both in phase to excite $\mathrm{Nc} 1$ and $\mathrm{Nc} 3$.

Case 2 place $4 u_{B}$ at NDE AMB location and $4 u_{B}$ at DE AMB location, $180^{\circ}$ out of phase to excite $\mathrm{Nc} 2$ and $\mathrm{Nc} 4$.

During the analysis the rotor displacement response at the NDE, DE and mid-span sensor locations as well as the perturbation current consumed by each bearing is plotted. The goal is to keep the rotor response within $30 \%$ of the air gap and the perturbation current less than $80 \%$ of the bias current over the entire range of operation. No specific guideline is provided by API or ISO with respect to the allowable current amplitude and bearing force during the unbalance response. The standards merely suggest that the worst case unbal- 
ance response remains within the power bandwidth of the actuator. Figures 5.29 and 5.30 provides the displacement at the sensor locations, amplifier current response and estimated bearing force delivered for both unbalance cases described above. The Benchmark I controller (Kdk_48_17Aug11a) was used to generate the responses shown. The displacement response in case 1 (see Figure 5.29a) the first bending mode Nc3 has been excited by the unbalance producing a response at the bearing locations that remains within the allowed clearance but exceeds the $30 \%$ recommendation while traversing the critical speed. The response due to unbalance distribution in case 2 (see Figure 5.30a) at Nc3 remains within the $30 \%$ clearance recommendation. Furthermore, the rigid body modes Nc1 and Nc2 all have well damped responses.

\subsubsection{Stability (Damped Mode Shape) Analysis}

During the stability analysis an eigenvalue analysis as outlined in Section 3.2.4 is carried out upon the closed-loop model consisting of the controller and nominal plant dynamics at the maximum operating speed. For this generalized eigenvalue problem, we cannot use the modally truncated rotor model as it lacks complete displacement information for rotor states. As in Equations (3.35) and (3.36), the solution produces complex eigenvalues (containing damping natural frequency information of the closed-loop system) and their associated eigenvectors (containing the relative displacement information of each station in the finite-element rotor model). The first 5 rotor modes $(\mathrm{Nc1}$ through $\mathrm{Nc5})$ and their forward and backward components were identified within the eigenvalue matrix, and associated lateral modal displacement in three or two dimensions computed. Figures 5.31 and 5.32 display the rotor mode shapes we are concerned about, as well as the damped natural frequency and logarithmic decrement. It is important that all rotor modes remain stable over the entire speed range, i.e., have a positive log decrement that indicates the presence of damping (Schmied et al., 1999). It is also important that the modal displacement occurring at the sensor and bearing locations shown in the figures, is sufficient to not only detect the 


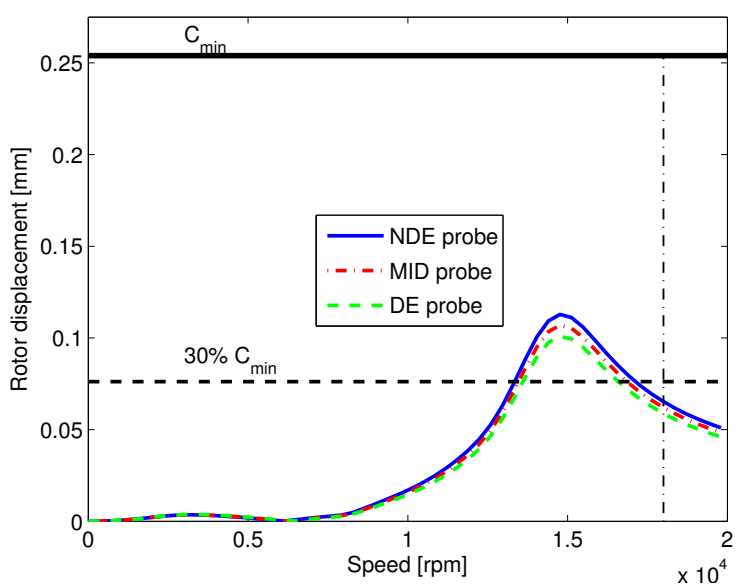

(a) Displacement at sensors.

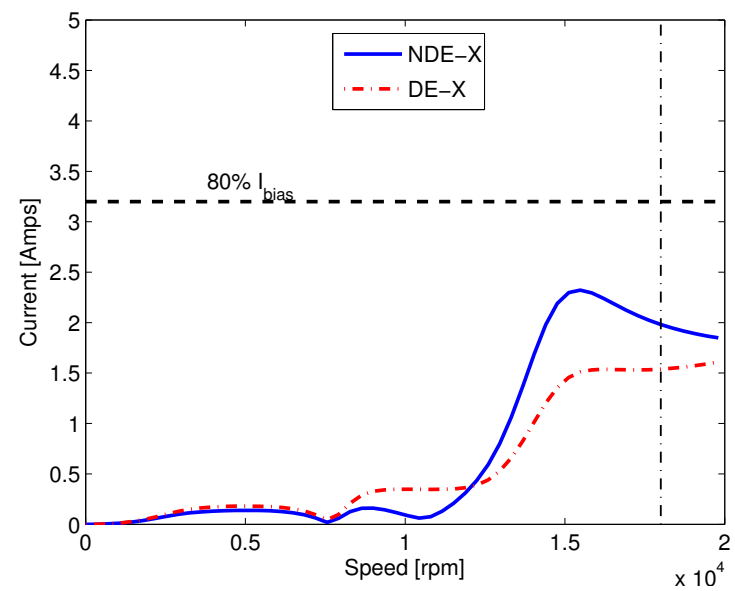

(b) Bearing current.

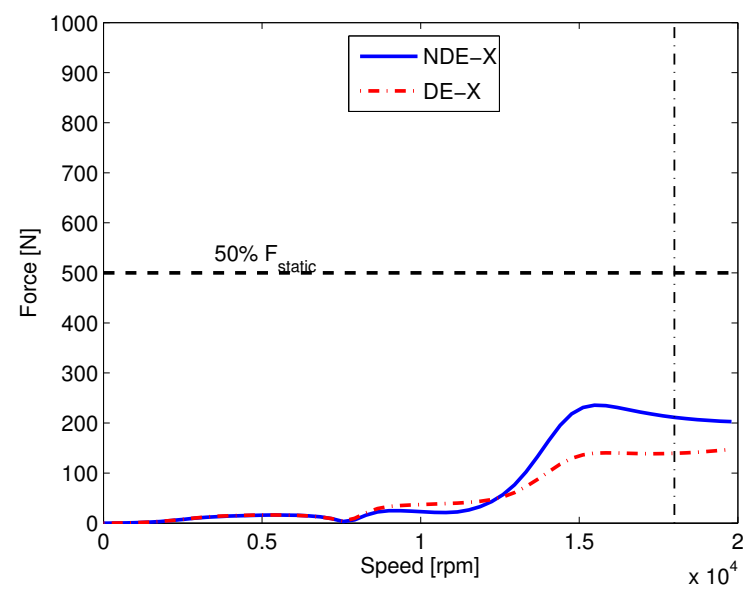

(c) Bearing force.

Figure 5.29: Unbalance response case 1 for Benchmark I controller 


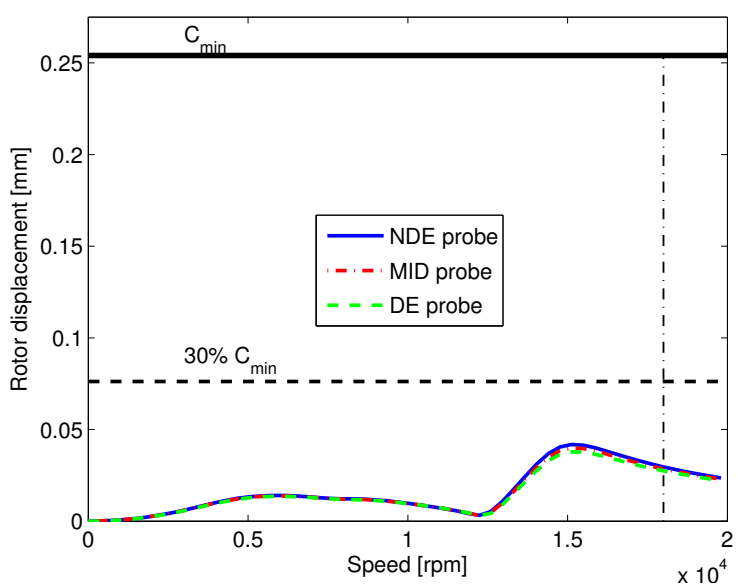

(a) Displacement at sensors.

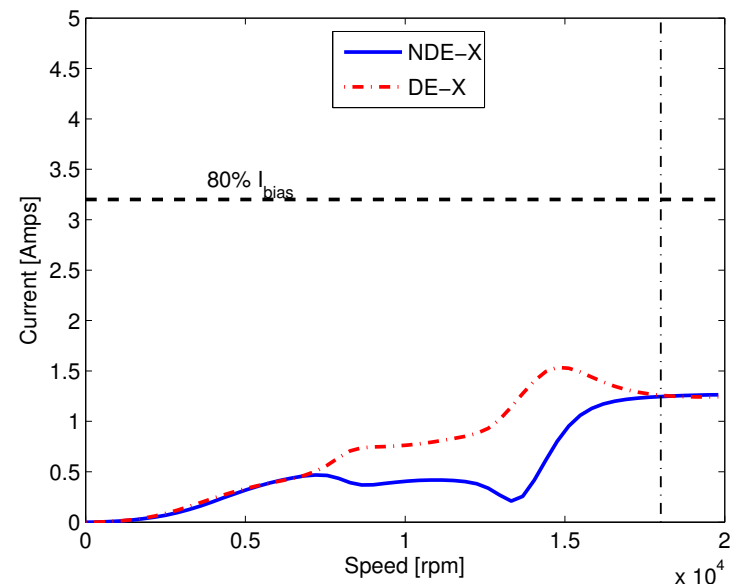

(b) Bearing current.

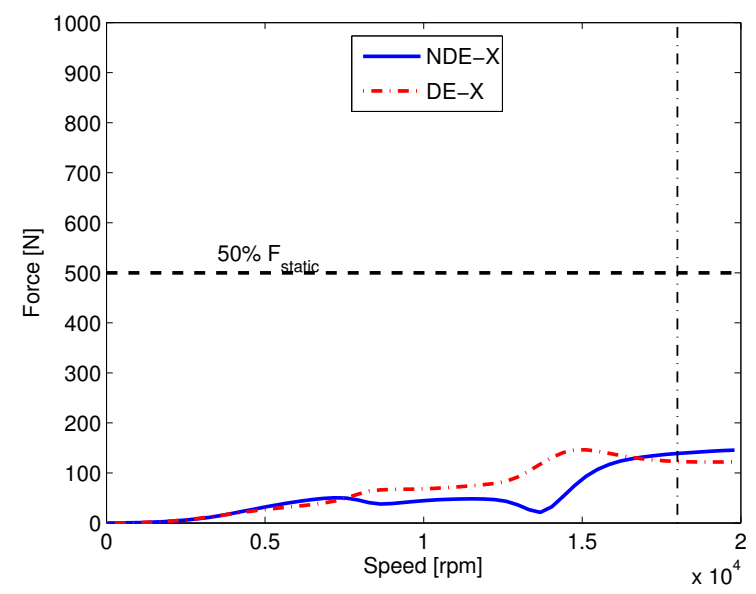

(c) Bearing force.

Figure 5.30: Unbalance response case 2 for Benchmark I controller 


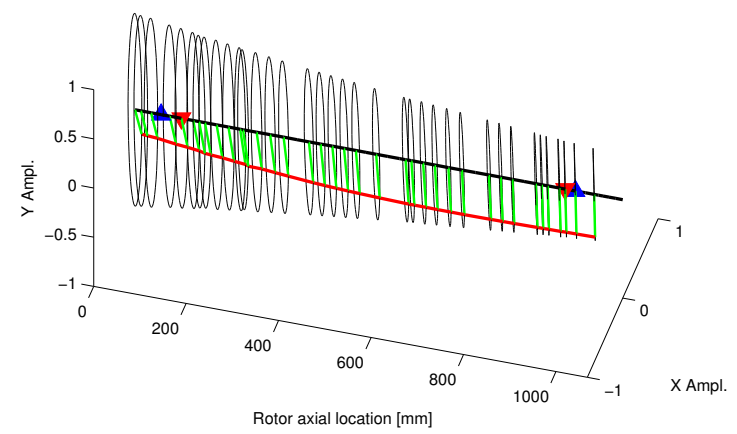

(a) Nc1 - Cylindrical - Backward.

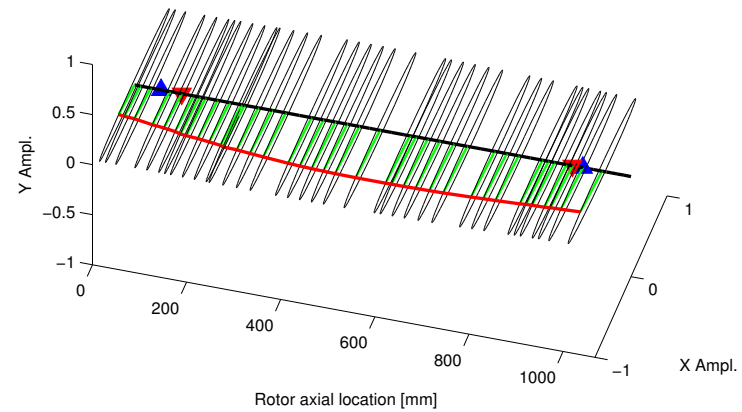

(b) Nc1 - Cylindrical - Forward.

Backward Mode, $\omega_{\mathrm{n}}=67.511 \mathrm{~Hz} / 4050.7 \mathrm{RPM} ;$ damping $=0.5514 ; \log$ dec $=4.15 ;$

Forward Mode, $\omega_{\mathrm{n}}=84.703 \mathrm{~Hz} / 5082.2 \mathrm{RPM} ;$ damping $=0.5434 ; \log$ dec $=4.067$

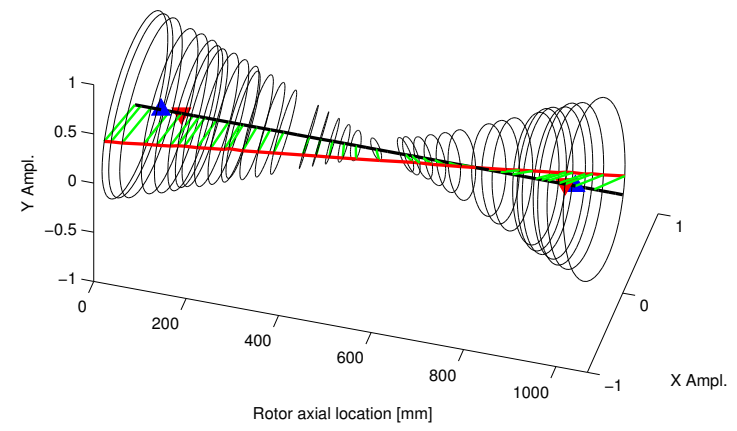

(c) Nc2 - Conical - Backward.

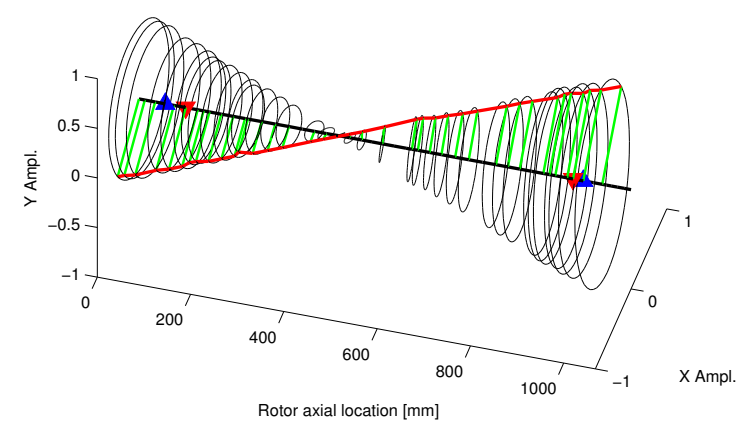

(d) Nc2 - Conical - Forward.

Figure 5.31: Predicted damped mode shapes of rotor rigid body modes with Benchmark I controller. The AMB locations are indicated by $\boldsymbol{\Lambda}$, and the displacement sensor locations are indicated by $\nabla$.

vibrations due to a resonant mode, but also damp them. The proximity of sensor or bearing to a nodal point for a bending mode significantly limits the observability and controllability of the mode.

\subsection{Summary}

In this chapter we have provided a motivation for the use of robust control to guarantee supercritical operation of the MBTRI experiment, and extend the stability threshold with respect to CCS. Identifying the uncertainties present and describing them in a form 


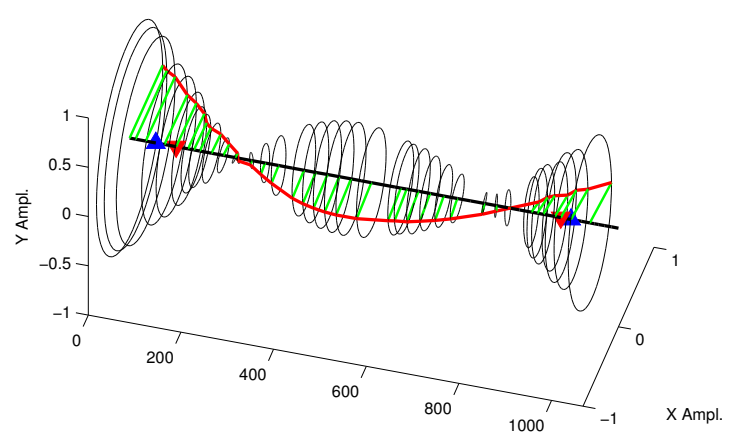

Rotor axial location [mm]

(a) Nc3 - First Bending - Backward.

Backward Mode, $\omega_{\mathrm{n}}=529.78 \mathrm{~Hz} / 31787 \mathrm{RPM} ;$ damping $=0.0321 ; \log$ dec $=0.202$

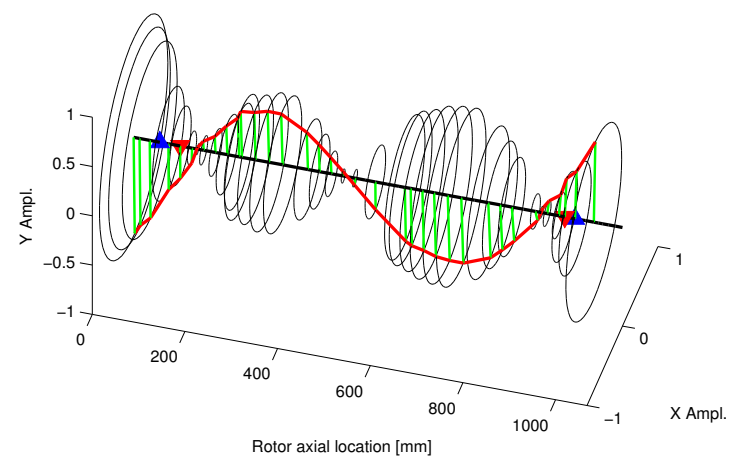

(c) Nc4 - Second Bending - Backward.

Mixed Mode, $\omega_{\mathrm{n}}=1080.1 \mathrm{~Hz} / 64804 \mathrm{RPM} ;$ damping $=0.0020 ; \log$ dec $=0.0123$

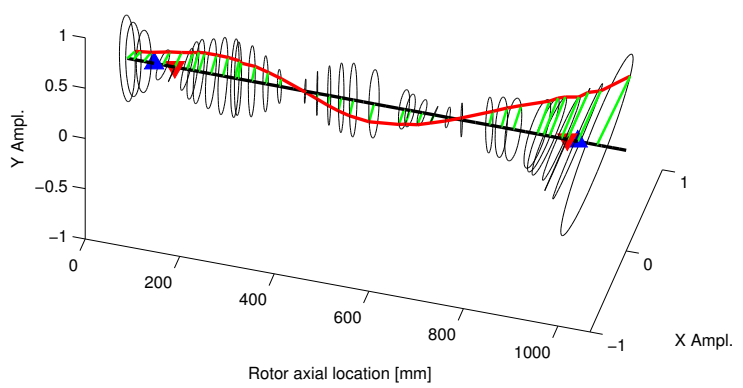

(e) Nc5 - Third Bending - Forward.

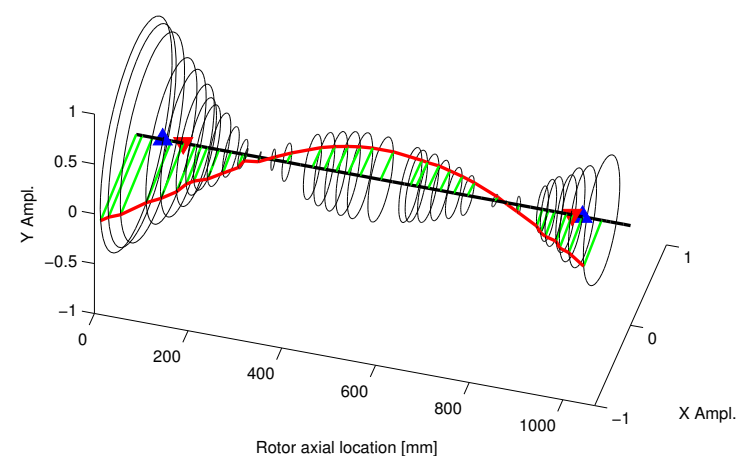

(b) Nc3 - First Bending - Forward.

Forward Mode, $\omega_{\mathrm{n}}=567.2 \mathrm{~Hz} / 34032 \mathrm{RPM} ;$ damping $=0.0203 ; \log$ dec $=0.1274$

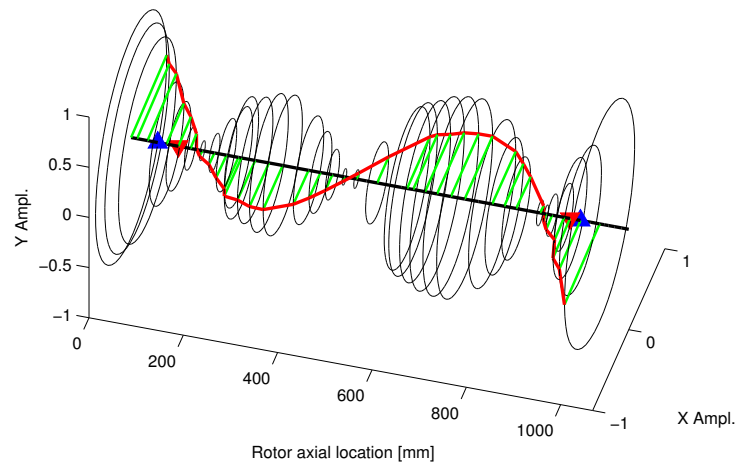

(d) Nc4 - Second Bending - Forward.

Backward Mode, $\omega_{\mathrm{n}}=979.2 \mathrm{~Hz} /$ 58752RPM ; damping $=0.0027 ; \log$ dec $=0.017$ :

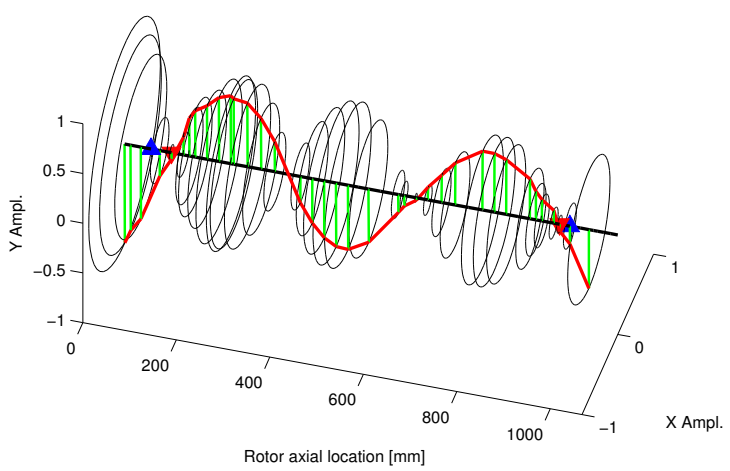

(f) Nc5 - Third Bending - Backward.

Figure 5.32: Predicted damped mode shapes of rotor bending modes with Benchmark I controller. The AMB locations are indicated by $\boldsymbol{\Lambda}$, and the displacement sensor locations are indicated by $\nabla$. 
amenable to inclusion in the nominal state-space model is performed. Frequency domain criteria for the closed-loop performance specifications are developed based on engineering objectives and the fundamental limitations discussed in earlier chapters. The structured singular value $\mu$ provides the machinery to synthesize and analyze multivariable robust controllers. and used to synthesize several different control laws. Simulations of the worst case unbalance response and closed-loop stability predictions were carried in accordance with industry guidelines. The next step is to confirm the validity of these theoretical results through experimentation. 


\section{Chapter 6}

\section{Experimental Results and Discussion}

The investigation presented in this dissertation on the application of robust control to extend the stability threshold with respect to aerodynamic loading satisfies the criteria for a well designed and meaningful experiment (Knospe \& Maslen, 1997). The major experimental results presented are the successful supercritical operation of the rotor, and the successful increase in the stability threshold by a $\mu$-synthesis controller developed in the previous chapter. In addition to these results, several system transfer functions for the benchmark controller are measured and compared to the inverse weighting function bounds used during synthesis. A general discussion of the stability threshold results within the context of trade off that exists in feedback control implementations between performance and robustness, and the optimum damping derivations discussed from the mechanical vibrations viewpoint highlighted in Chapter 3.

\subsection{Results}

\subsubsection{Supercritical Operation}

The combination of a bearing span to midspan diameter ratio of 15 and maximum operating speed of 18,000 rpm which is 1.3 times the first free-free bending mode (see Table 4.1) sug- 
gest the rotor will experience significant lateral deflection on traversing Nc3. Exploratory tests to find the maximum speed attained with the Benchmark I controller confirmed sensitivity of the rotor to imbalance. During a trial run on the approach to Nc3 at 14,000 rpm, high levels of synchronous rotor vibration (exceeding $70 \mu \mathrm{m}$ in amplitude at the DE sensing plane, and $51 \mu \mathrm{m}$ amplitude at the NDE sensing plane ${ }^{1}$ ) were accompanied by perturbation currents at the NDE AMB close to twice the bias current, i.e., limit enforced for linear operation of bearing (see Figure 6.1). Beyond 14,300 rpm the current requested by the controller exceeding the set limits for the power amplifier leading to a clipped output current. The distortion that results from this saturation nonlinearity introduces additional delay into the closed-loop negatively affecting its stability. As a result the controller can no longer constrain the rotor to a tight orbit and contact results between the rotor and stator. This observation combined with failure of successive attempts to increase the effective damping of the controller enough to traverse $\mathrm{Nc} 3$, led to the conclusion that high levels of rotor imbalance was causing an excessive controller response. A mechanical solution in the form of high-speed dynamic balancing was necessary to tackle the problem at its source.

The generalized two-plane influence coefficient method using data collected at multiple operating speeds was chosen based on its ease of use, and the fact that a knowledge of the rotor mode shapes was not required (Ehrich, 2004). The two discs attached to the rotor (labeled $\mathrm{c}$ and e in Figure 4.2b) served as the pair of balance planes, while the NDE and DE displacement sensors served as the response measurement locations. The magnitude and phase of the synchronous (1X) component of the displacement signals were computed by the DSP using an optical once-per-revolution sensor as a reference. The measurements were taken at three speeds prior to observed increases in vibration amplitude, i.e., 3,900 rpm, 7,500 rpm and 12,162 rpm, and the BALOPT balancing code based on second-order

\footnotetext{
${ }^{1}$ In addition to high rotor vibration amplitudes measured by the sensors close to the rotor natural frequencies, occupants of the Mechanical Engineering building on several floors above the MBTRI experiment noted vibrations while the test rig operated in the vicinity of $9,000 \mathrm{rpm}$. As there is no rotor natural frequency close to $150 \mathrm{~Hz}$, the author and colleagues believe this vibration to be the result of the excitation of structural building mode by the rotor imbalance force.
} 


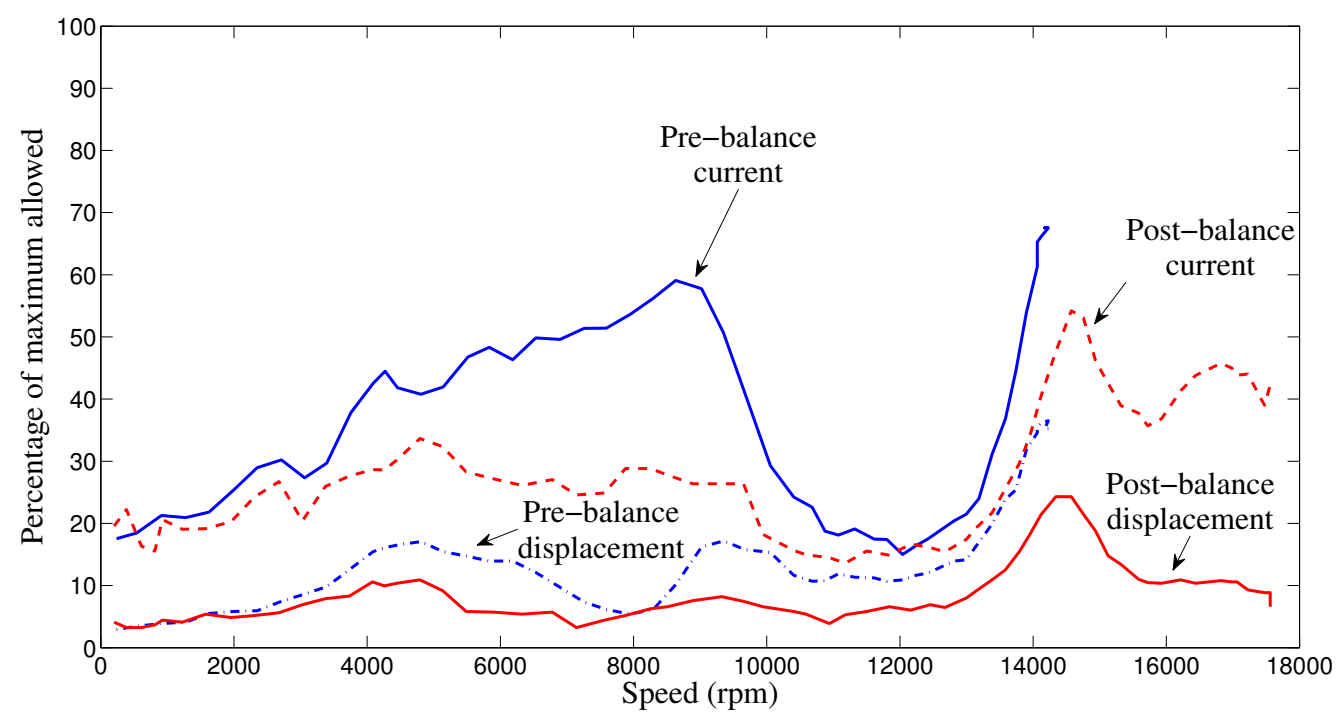

Figure 6.1: Rotor displacement (0-pk) and perturbation current at the NDE AMB as a percentage of the minimum clearance and bias current, respectively during a run-up to 18,000 rpm. Measurements are taken both before and after dynamic balancing.

cone programming was used to calculate optimal correction weights given constraints on the available balance holes and maximum balance weight (Huang, 2007). Table 6.1 shows the measured vibration response of the rotor prior to balancing, the vibration response following the addition of each trial weight, and the final response following the optimal correction weight calculated by BALOPT. After dynamic balancing, the rotor operated up to $18,000 \mathrm{rpm}$ with significantly lower perturbation current and orbit amplitudes ${ }^{2}$. For example the synchronous vibration amplitude at the DE and NDE sensors was reduced to $11 \mu \mathrm{m}$ and $15 \mu \mathrm{m}$, respectively. This represented a seven-fold reduction in the vibration approaching Nc3.

\subsubsection{System Transfer Functions}

Several system transfer functions were measured for the purpose of evaluating the performance of the closed-loop system stabilized by Benchmark controller I. The block diagram in Figure 6.2 illustrates several locations in the feedback control system where stimuli from

\footnotetext{
${ }^{2}$ Following successful dynamic balancing of the rotor, the vibration amplitude at 9,000 rpm decreased significantly and the building occupants on other floors were less aware of the MBTRI operation.
} 
Table 6.1: Two-plane influence coefficient balancing results.

\begin{tabular}{|c|c|c|c|c|c|}
\hline & $\begin{array}{l}\text { Balance } \\
\text { mass } \\
\text { at BP1 } \\
\left(g \angle^{\circ}\right)\end{array}$ & $\begin{array}{c}\text { Balance } \\
\text { mass } \\
\text { at BP2 } \\
\left(g \angle^{\circ}\right)\end{array}$ & $\begin{array}{c}1 \mathrm{X} \mathrm{DE} \\
\text { vibration } \\
{[0-\mathrm{pk}]} \\
\left(\mu \mathrm{m} \angle^{\circ}\right)\end{array}$ & $\begin{array}{c}1 \mathrm{X} \text { NDE } \\
\text { vibration } \\
{[0-\mathrm{pk}]} \\
\left(\mu \mathrm{m} \angle^{\circ}\right)\end{array}$ & $\begin{array}{l}\text { Rotational } \\
\text { speed } \\
\text { (rpm) }\end{array}$ \\
\hline \multirow{3}{*}{ Initial } & & & $22.2 \angle 267$ & $49.8 \angle 182$ & 3,900 \\
\hline & & & $16.8 \angle 185$ & $13.4 \angle 141$ & 7,500 \\
\hline & & & $71.5 \angle 164$ & $50.4 \angle 128$ & 12,160 \\
\hline \multirow{3}{*}{ Trial mass \#1 } & \multirow{3}{*}{$1.23 \angle 57$} & & $24.4 \angle 282$ & $35.4 \angle 170$ & 3,900 \\
\hline & & & $28.6 \angle 188$ & $4.2 \angle 44$ & 7,500 \\
\hline & & & $62.7 \angle 176$ & $35.1 \angle 154$ & 12,160 \\
\hline \multirow{3}{*}{ Trial mass \#2 } & & \multirow{3}{*}{$1.23 \angle 120$} & $21.6 \angle 266$ & $46.4 \angle 170$ & 3,900 \\
\hline & & & $14.7 \angle 174$ & $12.7 \angle 144$ & 7,500 \\
\hline & & & $56.2 \angle 160$ & $46.7 \angle 127$ & 12,160 \\
\hline \multirow{3}{*}{ Correction mass } & \multirow{3}{*}{$0.888 \angle 103$} & \multirow{3}{*}{$3.52 \angle 90$} & $12.5 \angle 206$ & $23.6 \angle 155$ & 3,900 \\
\hline & & & $6.9 \angle 285$ & $14.1 \angle 114$ & 7,500 \\
\hline & & & $11.0 \angle 157$ & $15.5 \angle 156$ & 12,160 \\
\hline
\end{tabular}

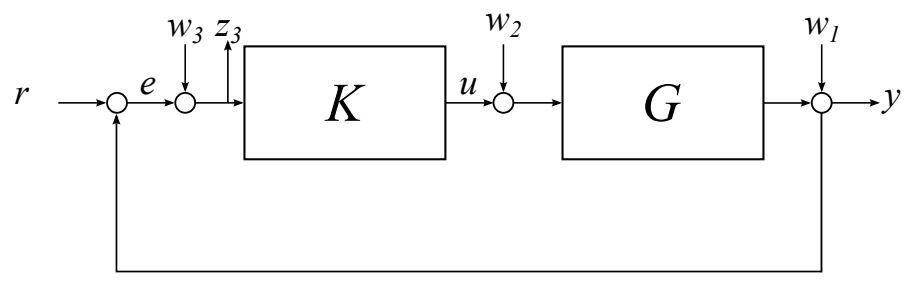

Figure 6.2: Block diagram showing stimulus and response points for system transfer function measurement. 
Table 6.2: Gain and phase margins from first loop gain crossover of the diagonal channels using Benchmark I controller.

\begin{tabular}{|c|c|c|c|c|}
\hline & $\begin{array}{c}\text { Gain crossover } \\
\text { frequency }(\mathrm{Hz})\end{array}$ & $\begin{array}{c}\text { Phase crossover } \\
\text { frequency }(\mathrm{Hz})\end{array}$ & $\begin{array}{c}\text { Gain Margin } \\
(\mathrm{dB})\end{array}$ & $\begin{array}{c}\text { Phase Margin } \\
\left({ }^{\circ}\right)\end{array}$ \\
\hline \hline NDEX & 45.8 & 169 & 10.3 & 27 \\
\hline DEX & 71 & 175 & 11.0 & 27 \\
\hline NDEY & 45.8 & 181 & 10.6 & 27 \\
\hline DEY & 71 & 197 & 12.2 & 27 \\
\hline
\end{tabular}

a system analyzer may be injected, and the response measured to give an experimental frequency response. The first and perhaps most informative of which is the loop gain transfer function $L(j \omega)=G(j \omega) K(j \omega)$, which is the open-loop response from controller input to plant output, i.e., $z_{3}$ to $y$ with stimulus on $w_{3}$. Bode plots of $L(j \omega)$ are used extensively in classical controller design to allow figures of merit such as the gain and phase margins to be deduced graphically from the crossover points. Open-loop gains for rotor-AMBs systems are characterized by multiple zero crossings of $|L(j \omega)|$ hence multiple gain and phase margins may be quoted. The gain and phase margins of the first loop gain cross over frequency are presented in Table 6.2. These values agree with the lower bound on the gain and phase margins predicted by the available bandwidth calculation (see Section 4.5.1 on page 88). Additional crossovers also indicate whether a bending mode is amplitude or phase stabilized ( $\mathrm{Li}, 2006)$. The rigid body rotor modes are always phase stabilized. Figure 6.3 presents eight loop transfer functions measured one loop at a time, representing the block diagonal directions of the rotor-AMB system at $0 \mathrm{rpm}$ stabilized by the benchmark controller, i.e., all interactions except $x-y$.

As discussed in Section 5.4, the closed loop sensitivity functions are used to define nominal performance specifications during controller synthesis. These frequency bounds take the form of the inverse of the product of the weighting functions used to shape a given sensitivity function as in Equation (5.25). The nominal performance of the closedloop system can be determined through the experimental analysis of several sensitivity functions: 
- The output sensitivity $S(j \omega)$ at $0 \mathrm{rpm}$ along the four channels was measured one loop at a time (gain from $w_{1} \rightarrow y$ with stimulus at $w_{1}$ ) and is shown in Figure 6.4. $S(j \omega)$ was also measured with the rotor spinning at 5,000 rpm and 10,000 rpm to confirm the robustness of the controller to changes in the running speed as shown in Figure 6.5. Both sets of plots demonstrate high level of disturbance rejection at low frequencies and the peak output sensitivity is less than the $9.5 \mathrm{~dB}$ upper bound defined by the inverse weighting function $\left(W_{p} W_{r}\right)^{-1}$. Furthermore, this peak sensitivity is within ISO 14839-3 Zone A/B, one indicator of satisfactory stability robustness (ISO 14839-3, 2006). The peak sensitivity region coincides with rigid body modes suggesting a variation of the actuator gain property, i.e., $K_{i}$ and $K_{x}$, from their expected values. Sharp peaks around bending modes have been largely avoided for they are indicative of poor robustness because slight perturbation in bending mode frequency may lead to large change in sensitivity. The peak sensitivity $S_{\max }$ determined from measurements across all the control channels was $9.17 \mathrm{~dB}$ or 2.87. Applying the SISO formulas presented in Chapter 3 indicates minimum gain and phase margins of $3.7 \mathrm{~dB}$ and $20^{\circ}$.

- The complementary sensitivity function $T(j \omega)$ shown in Figure 6.6 indicates reduced sensitivity of the control output to plant output disturbances and measurement noise entering the feedback path. The function was obtained by measuring the response from $r \rightarrow y$ with a stimulus at $r$. The closed-loop bandwidth $\omega_{B}$ of each control channel is obtained from the unity gain crossover frequency. The bandwidths for the NDE-X, DE-X, NDE-Y and DE-Y channels are 89 Hz, $110 \mathrm{~Hz}, 94 \mathrm{~Hz}$, and 105 Hz, respectively.

- The process sensitivity function $G S(j \omega)$ can be interpreted as the mechanical compliance of the closed-loop system. At approximately $30 \mathrm{~Hz}$ the bound $\left(W_{p} W_{d}\right)^{-1}$ was exceeded indicating the controller provided lower than expected stiffness in vicinity 


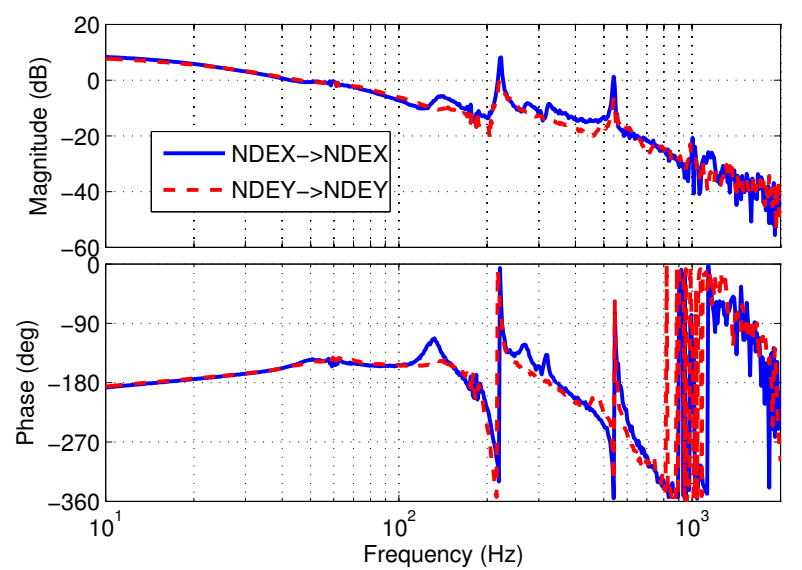

(a)

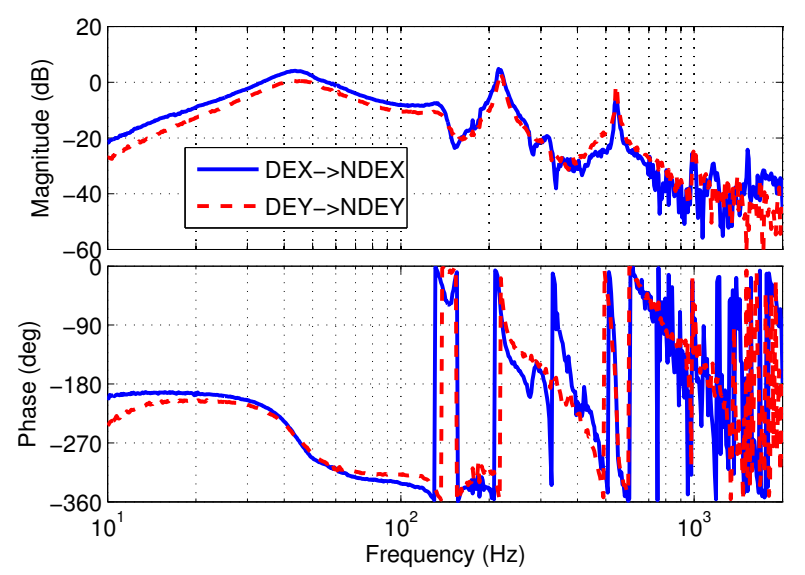

(c)

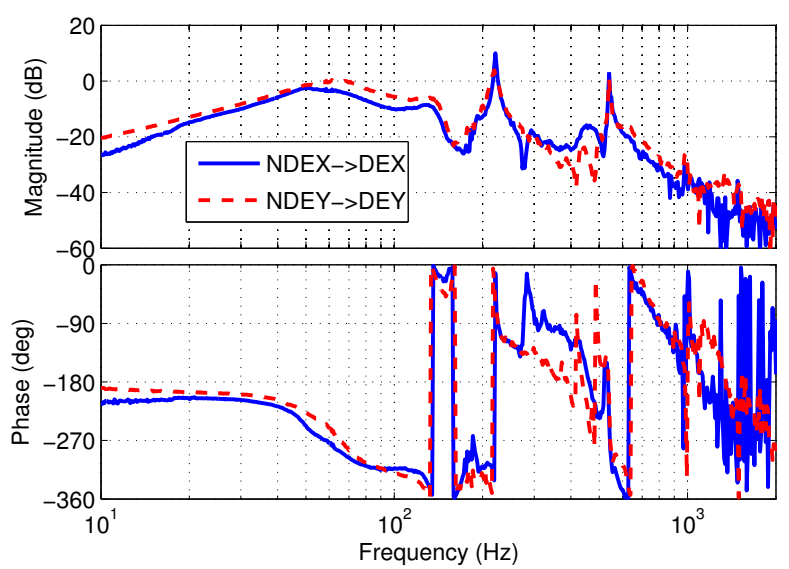

(b)

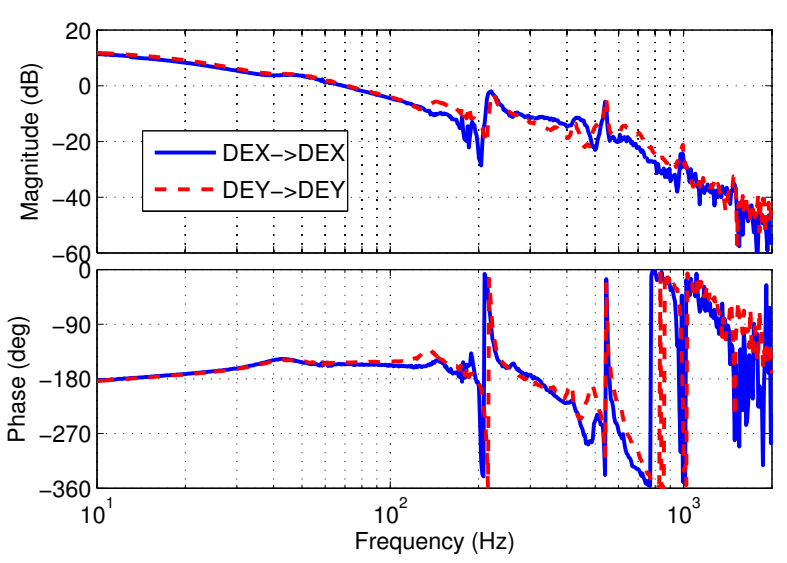

(d)

Figure 6.3: Bode plots of measured loop transfer functions.

of the first rigid body mode (see Figure 6.7). This is likely due to uncertainty in $K_{x}$ and/ or $K_{i} . G S(j \omega)$ was determined by measuring the response from $w_{2} \rightarrow y$ with an excitation at $w_{2}$.

- The control sensitivity $K S(j \omega)$ was obtained by multiplying the controller response for a given channel by the measured sensitivity function. The plot shown in Figure 6.8 demonstrates satisfactory roll-off of the control action at high frequencies, which is essential to prevent the excitation of unmodeled dynamics and the deterioration of the feedback response due to amplification of measurement noise. 


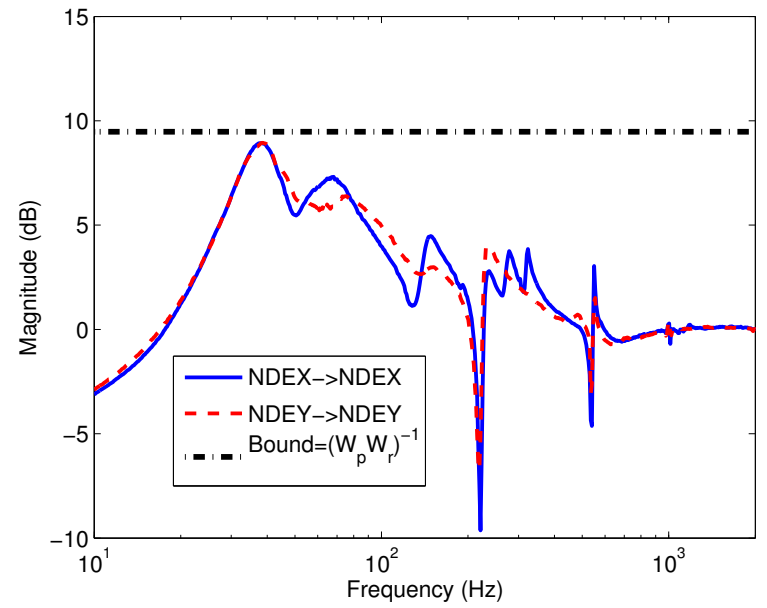

(a)

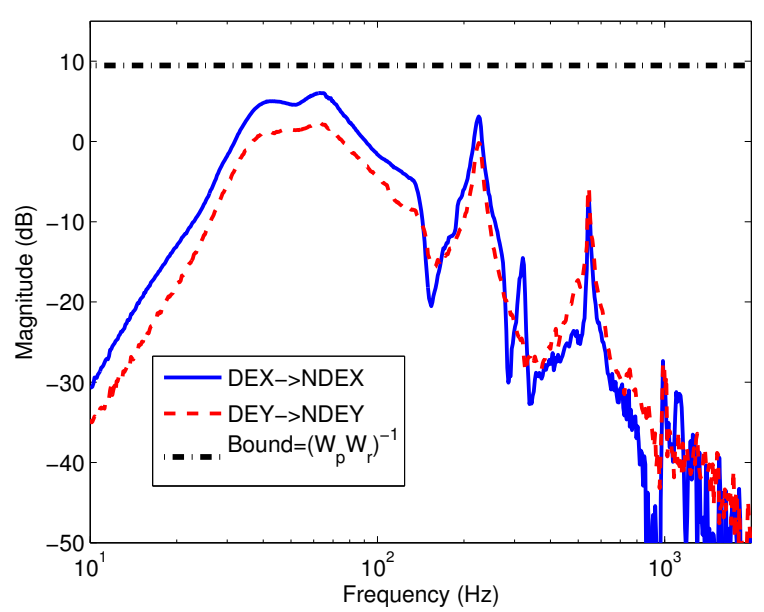

(c)

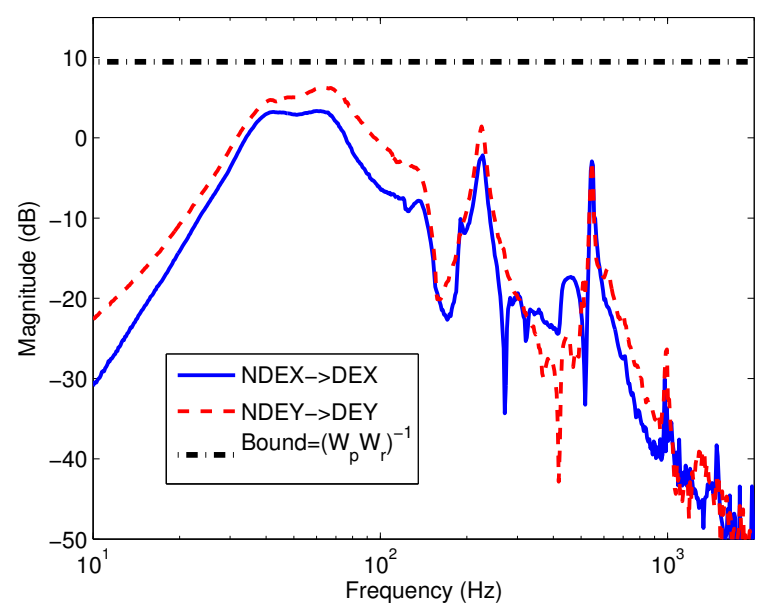

(b)

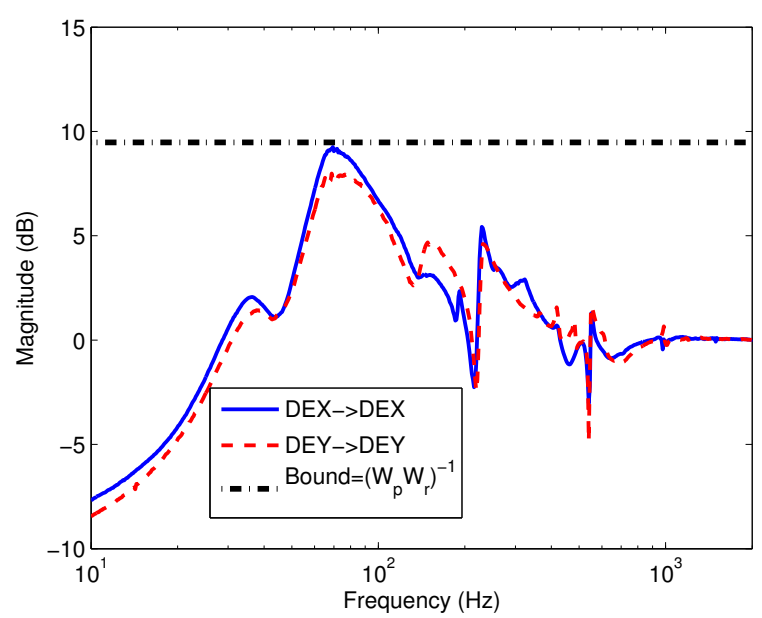

(d)

Figure 6.4: Bode plots of output sensitivity functions measured at $0 \mathrm{rpm}$. 


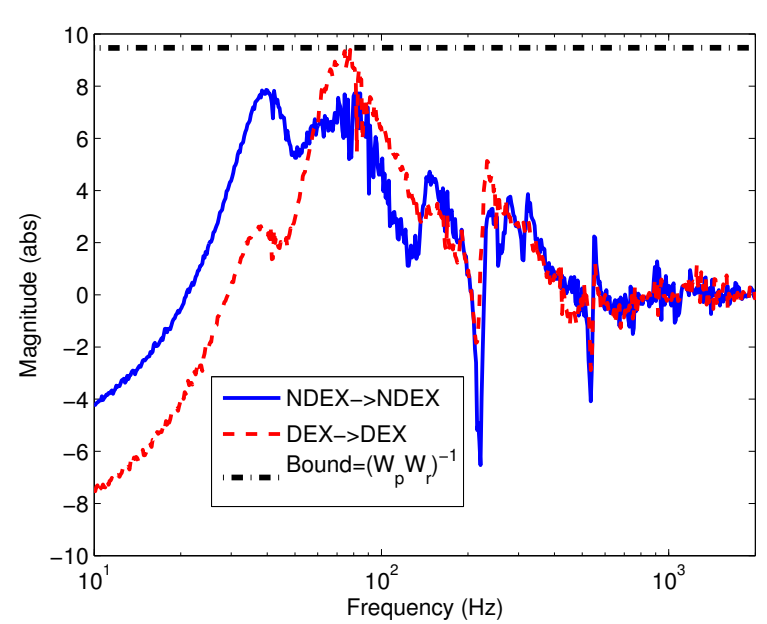

(a) $5,000 \mathrm{rpm}$

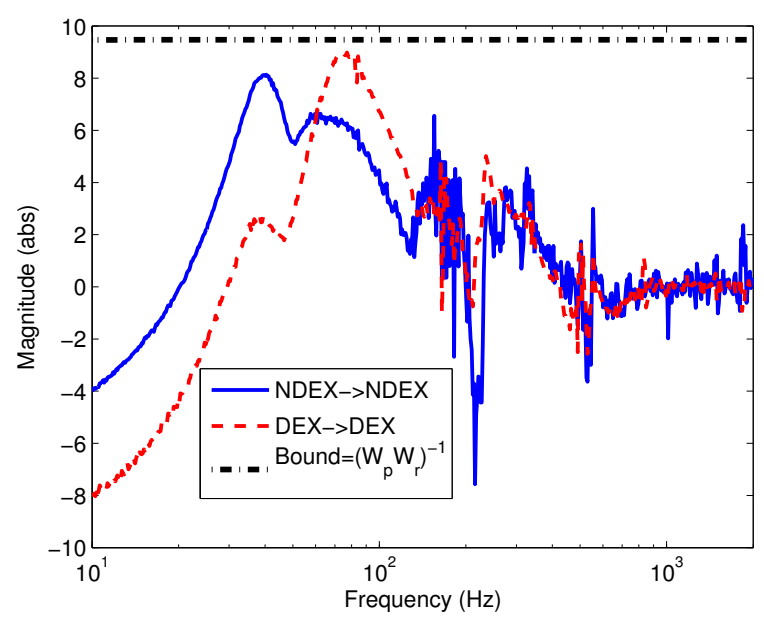

(b) $10,000 \mathrm{rpm}$

Figure 6.5: Bode plots of output sensitivity functions measured at various speeds.

\subsubsection{Stability Threshold}

The stability of the first rigid body rotor mode Nc1 is strongly affected by the destabilizing CCS added to the rotor-AMB system as was shown in Chapter 3. We are interested in determining which combination of performance weights and uncertainty models can maximize the magnitude of CCS required to drive the closed-loop unstable. Instability, as noted in Section 3.1 .1 on page 19 , is considered in the linear asymptotic case, i.e., once the damping ratio of $\mathrm{Nc1}$ becomes zero. The maximum value of CCS prior to the onset of instability is denoted the stability threshold.

To experimentally determine this threshold, successively higher levels of CCS were applied using the midspan AMB. The CCS reduces the damping of the eigenvalue corresponding to $\mathrm{Nc1}$ by encouraging forward whirl. The damping ratio of $\mathrm{Nc} 1 \mathrm{can}$ be estimated from the free decay of rotor vibration at the natural frequency of $\mathrm{Nc1}$. As the controller is constantly acting to attenuate any vibration in the system, an external excitation is required to bring about a measure decay response. Two forms of external excitation: impulse and circular sinusoidal were applied to the rotor. The impulse was delivered to the large disc on the rotor by a rubber-tipped modal impact hammer. The angle of impact was either ver- 


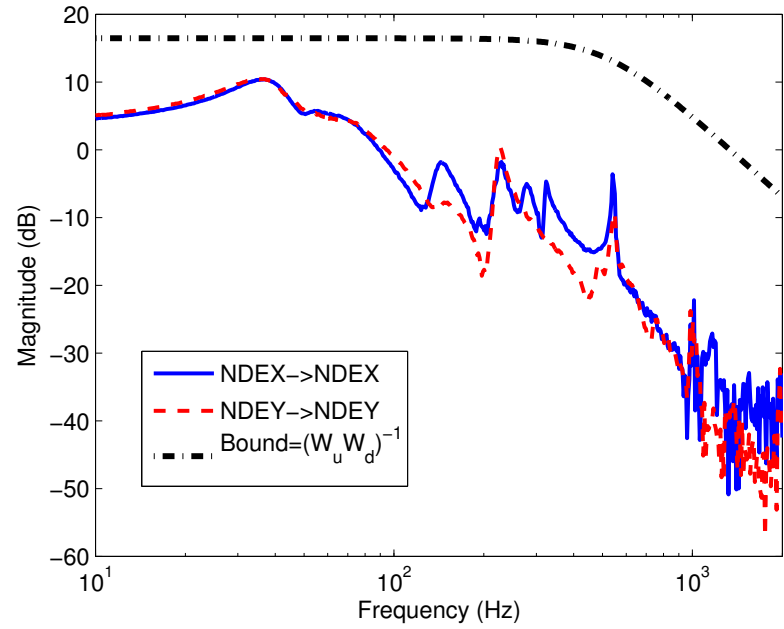

(a)

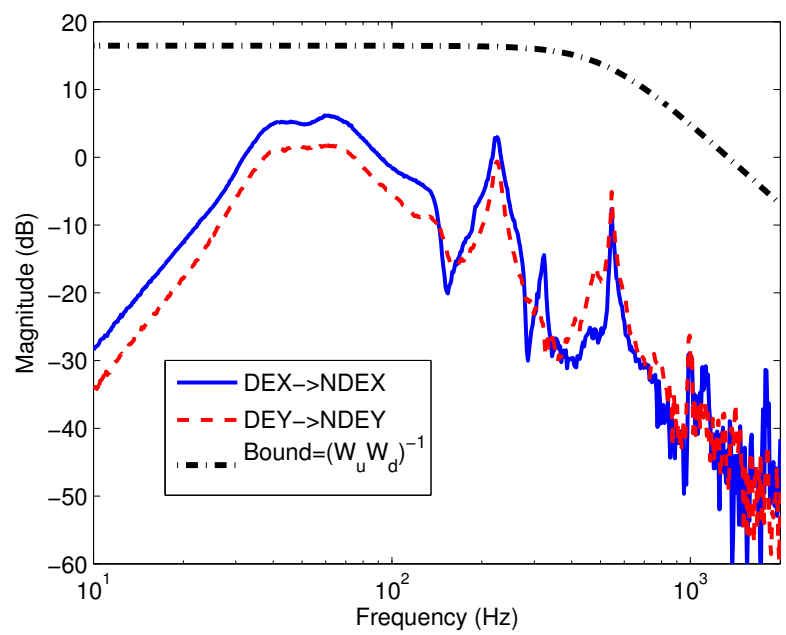

(c)

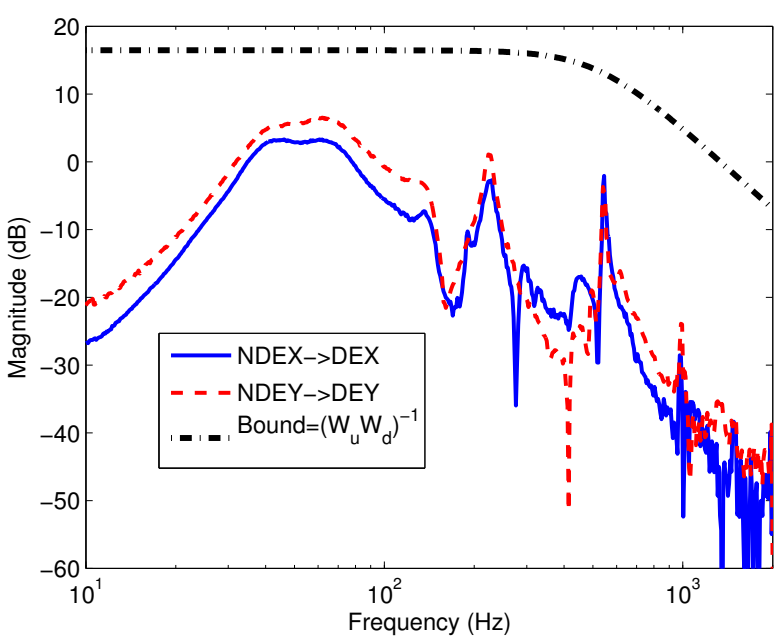

(b)

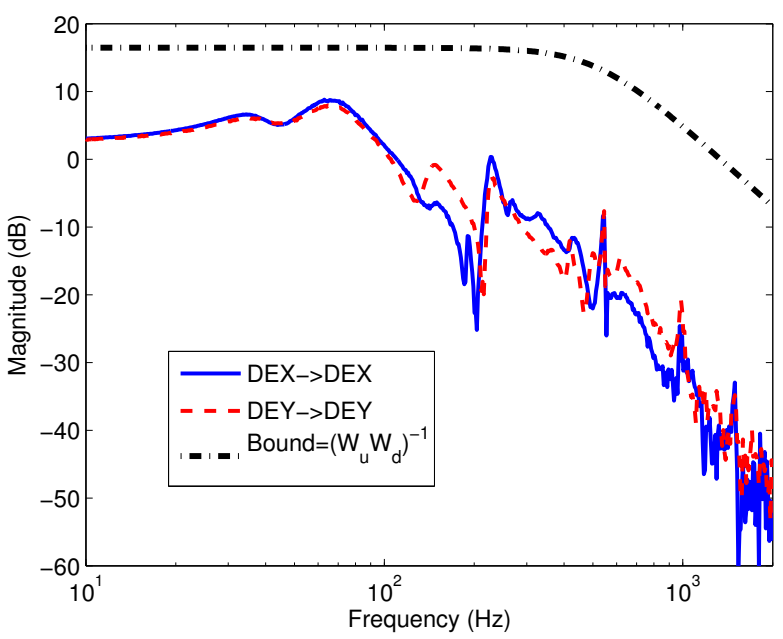

(d)

Figure 6.6: Bode plots of measured complementary sensitivity function or closed-loop response. 


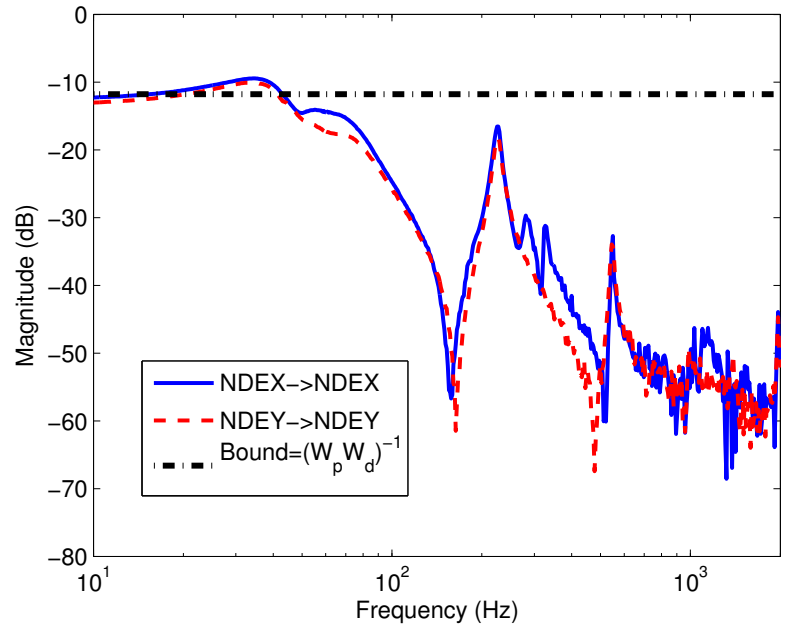

(a)

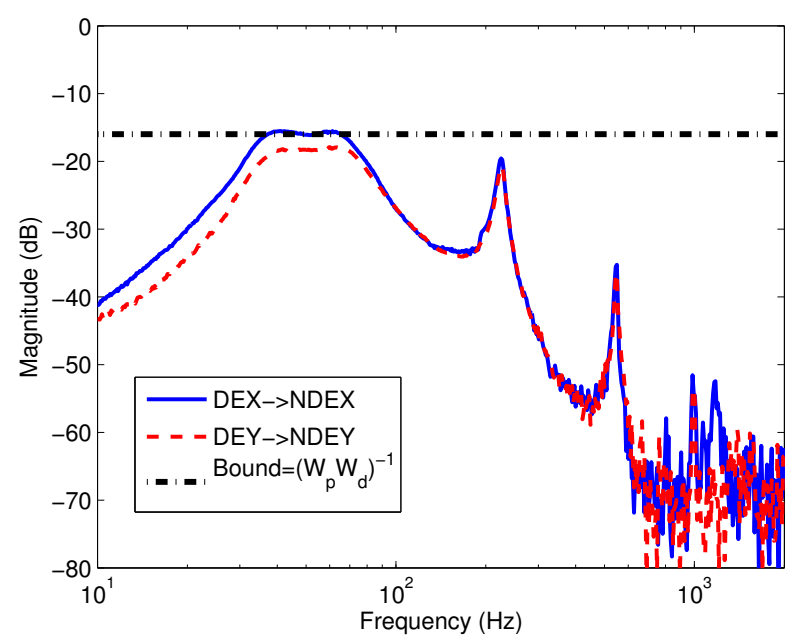

(c)

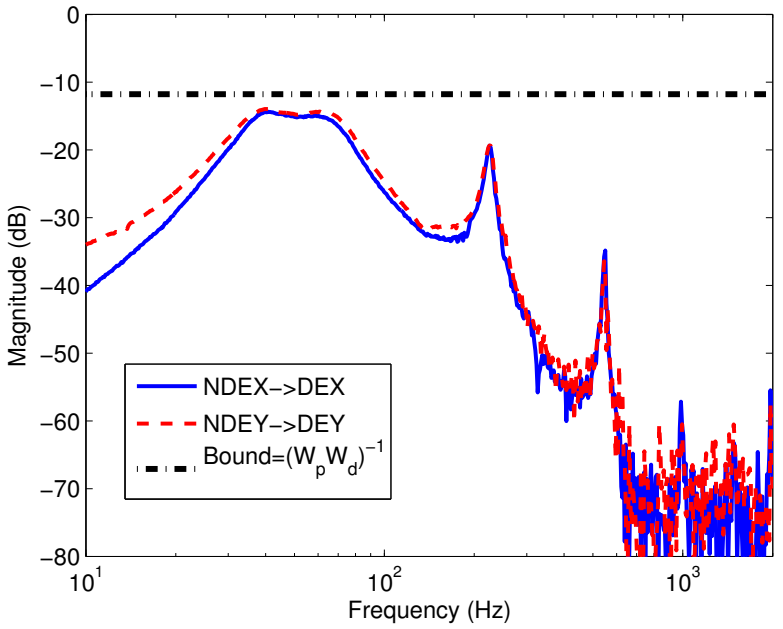

(b)

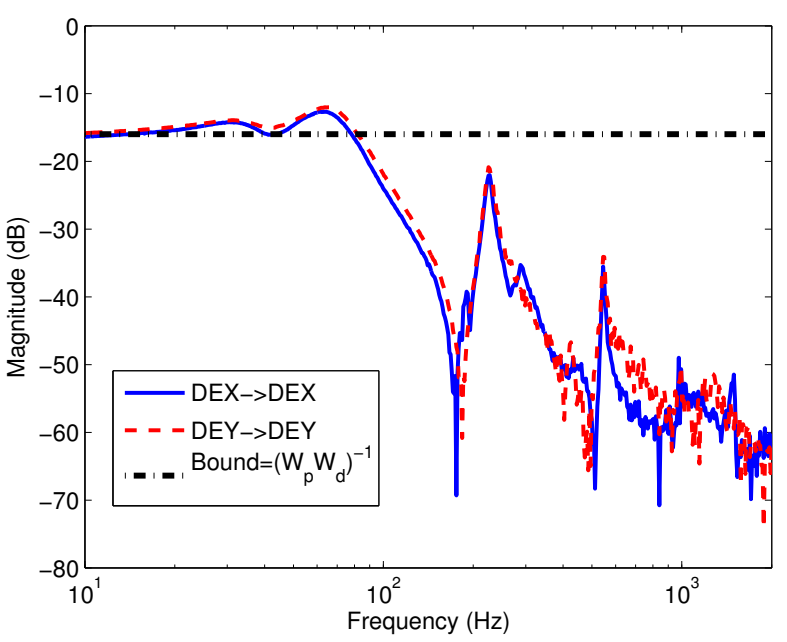

(d)

Figure 6.7: Bode plots of measured process sensitivity function. 


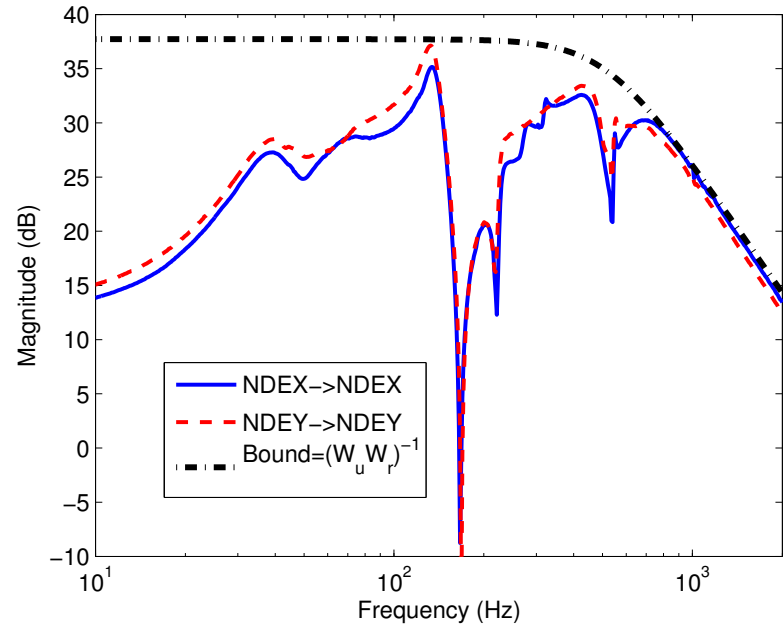

(a)

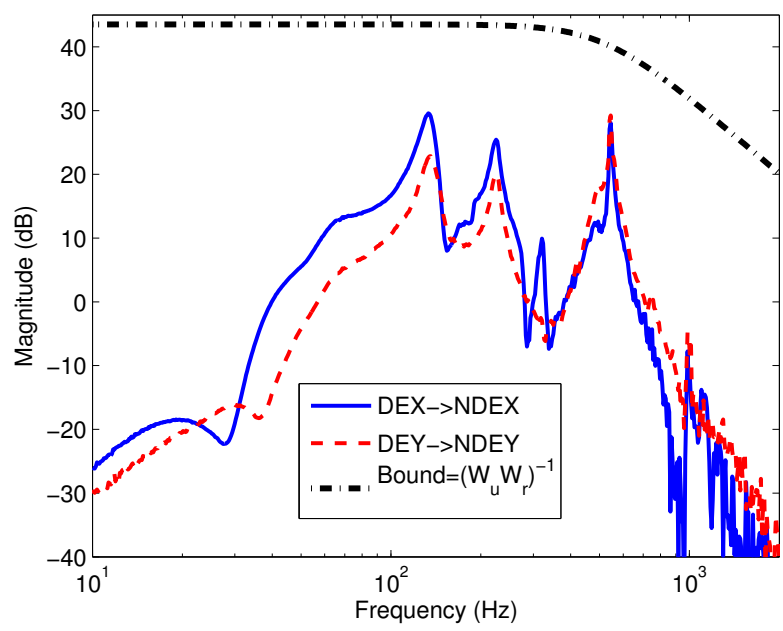

(c)

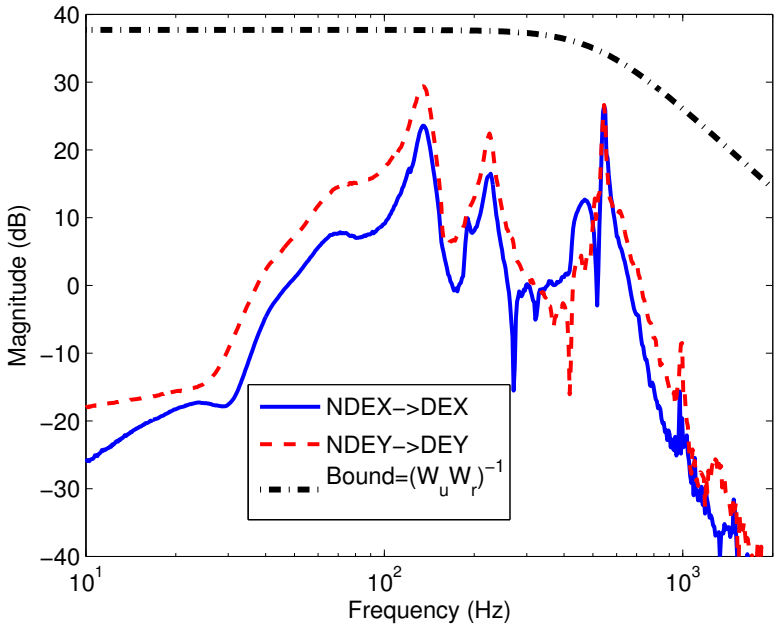

(b)

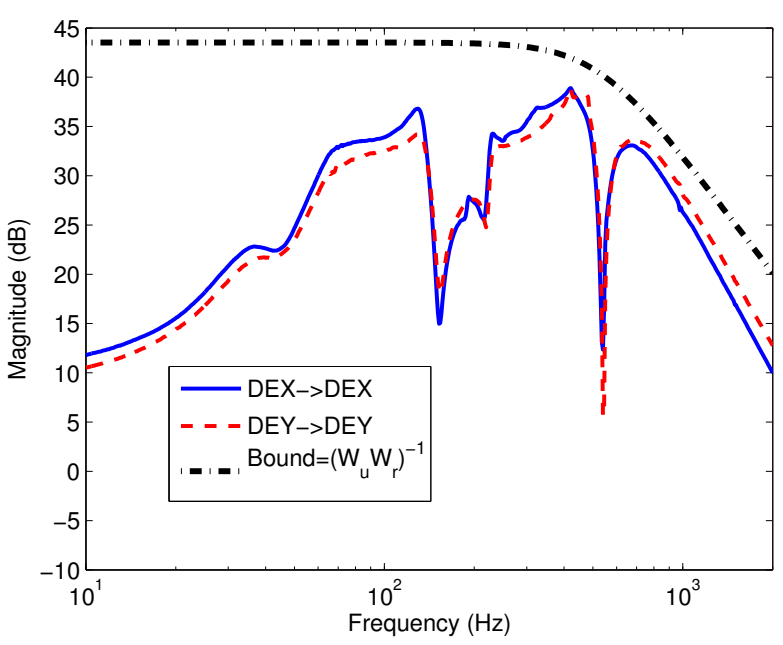

(d)

Figure 6.8: Bode plots of measured control sensitivity function. 
tical, $-45^{\circ}$ from vertical, i.e., aligned with $x$-axis of the AMB, or $+45^{\circ}$ from vertical, i.e., aligned with $y$-axis of the AMB. The vertical impact excites both the channels equally, however, the other impact directions are useful in determining the effects of support stiffness anisotropy on stability. Circular sinusoidal excitation was provided by driving the quarter span radial AMB in open-loop mode. The excitation frequency and direction (forward or backward) was varied so as to target the precise frequency of Nc1. This is known as blocking (Cloud et al., 2009). The free decay of the rotor vibrations following either the hammer impact, or termination of the blocking excitation was recorded from multiple position sensors using a separate data acquisition system from the DSP controller (see Appendix C). The time-domain data was processed offline using a backward auto-regression algorithm written to extract the natural frequency and damping information (Kumaresan \& Tufts, 1982).

Figure 6.9 shows the rotor displacement response at a single position sensor on the termination of a blocking excitation (indicated by the rising edge trigger shown on the plot) while the rotor is spinning at 7,000 rpm. At high levels of CCS, the distinct exponential amplitude decay of $\mathrm{Nc} 1$ is visible, and is quite easily extracted by the damping ratio algorithm. As the controller strongly damps $\mathrm{Nc1}$ at low levels of CCS, the decay is short lived and the SDOF damping ratio estimation algorithm has difficulty extracting this eigenvalue from the noisy background.

Table 6.3 shows the experimentally determined CCS threshold of the controllers designed in the previous chapter. The benchmark controller attained a stability threshold of $2000 \mathrm{~N} / \mathrm{mm}$. The minimum observed threshold, $730 \mathrm{~N} / \mathrm{mm}$, was achieved by the controller designed with 50\% support stiffness anisotropy and lacking an uncertainty model for CCS. The maximum threshold, $2700 \mathrm{~N} / \mathrm{mm}$ was obtained by the controller with block diagonal performance weights and using the CCS Model 3 uncertainty description. This maximum threshold represents an improvement of $36 \%$ over the benchmark controller.

It is necessary to understand the sensitivity of the rotor-bearing system stability to 


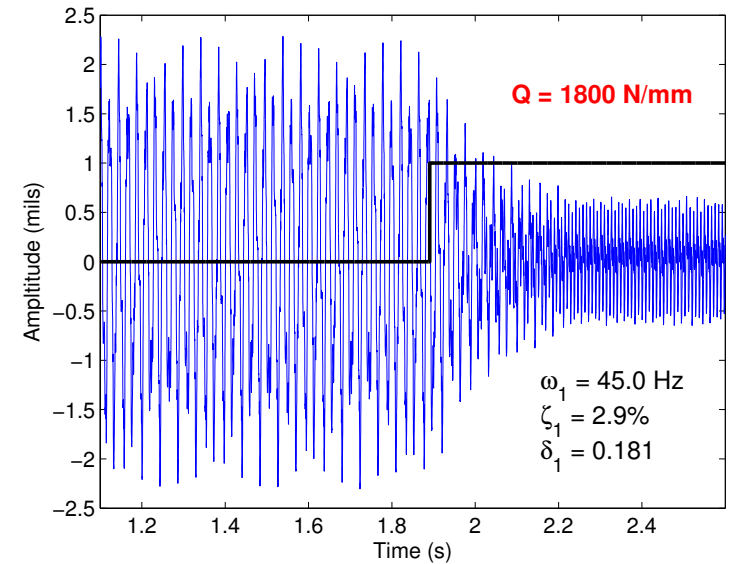

(a) $\mathrm{Q}=1800 \mathrm{~N} / \mathrm{mm}$

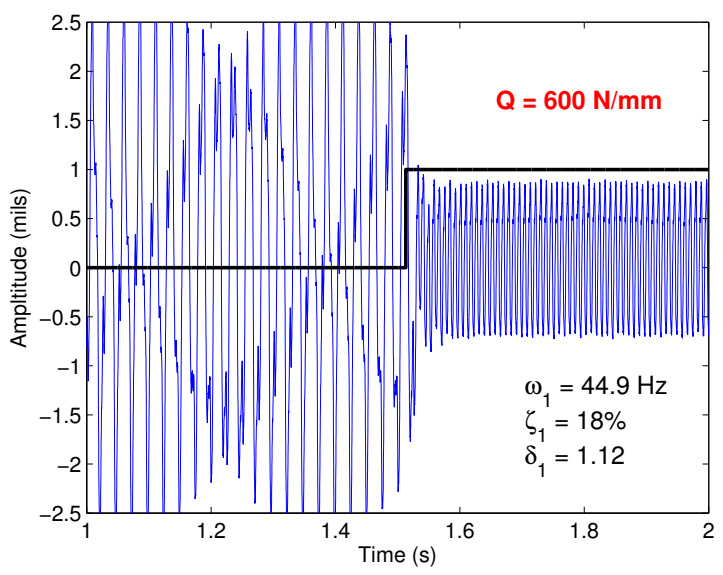

(c) $Q=600 \mathrm{~N} / \mathrm{mm}$

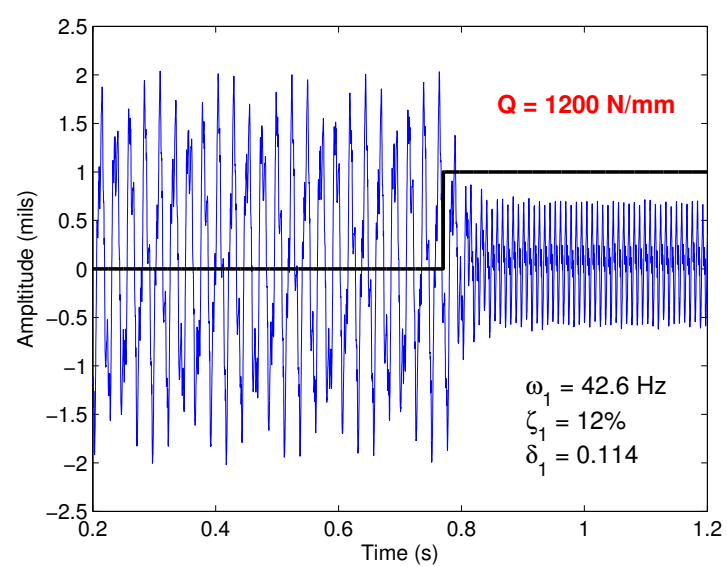

(b) $Q=1200 \mathrm{~N} / \mathrm{mm}$

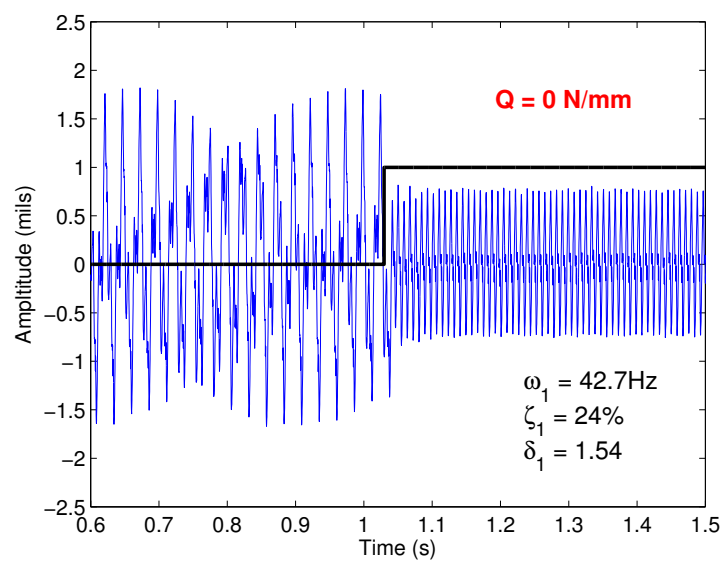

(d) $\mathrm{Q}=0 \mathrm{~N} / \mathrm{mm}$

Figure 6.9: Rotor displacement response at 7,000 rpm on termination of blocking (indicated by the rising edge trigger) under different magnitudes of CCS excitation. Benchmark I controller is used to suspend the rotor and the stability threshold for this controller is 2000 $\mathrm{N} / \mathrm{mm}$. 
Table 6.3: Experimental stability threshold of various controllers synthesized.

\begin{tabular}{|c|c|c|c|c|}
\hline \multirow{2}{*}{$\begin{array}{l}\text { Controller family } \\
\text { (file-name) }\end{array}$} & \multicolumn{2}{|c|}{ Uncertainty Model } & \multirow{2}{*}{$\begin{array}{c}\text { CCS Threshold } \\
(\mathrm{N} / \mathrm{mm})\end{array}$} & \multirow[t]{2}{*}{ Note } \\
\hline & Speed & CCS & & \\
\hline $\begin{array}{c}\text { Benchmark I } \\
\text { (Kdk_48_17Aug11a) }\end{array}$ & 2 & $\mathrm{n} / \mathrm{a}$ & 2000 & \\
\hline $\begin{array}{l}\text { 70\% Anisotropy I } \\
\text { (Kdk_48_16Jan12f) }\end{array}$ & 2 & $\mathrm{n} / \mathrm{a}$ & 2300 & \\
\hline $\begin{array}{c}\text { 50\% Anisotropy I } \\
\text { (Kdk_48_31Jan12a) }\end{array}$ & 2 & $\mathrm{n} / \mathrm{a}$ & $\begin{array}{c}2300 \\
730\end{array}$ & $\dagger$ \\
\hline $\begin{array}{c}\text { Full block } W_{p, 1} \\
\text { (Kdk_48_31Jan12k) }\end{array}$ & 2 & $\mathrm{n} / \mathrm{a}$ & 1300 & \\
\hline $\begin{array}{l}\text { Cross-coupled } W_{p, 2} \\
\text { (Kdk_31Jan12c) }\end{array}$ & 2 & $\mathrm{n} / \mathrm{a}$ & 1900 & \\
\hline $\begin{array}{l}\text { Block diagonal } W_{p, 3} \\
\text { (Kdk_52_01Feb12a) }\end{array}$ & 2 & $\mathrm{n} / \mathrm{a}$ & 2300 & \\
\hline $\begin{array}{l}\text { Block diagonal } W_{p, 4} \\
\text { (Kdk_48_31Jan12d) }\end{array}$ & 2 & $\mathrm{n} / \mathrm{a}$ & 2600 & \\
\hline $\begin{array}{l}\text { Block diagonal } W_{p, 5} \\
\text { (Kdk_48_31Jan12e) }\end{array}$ & 2 & $\mathrm{n} / \mathrm{a}$ & 1400 & \\
\hline $\begin{array}{c}\text { Benchmark Ic1 } \\
\text { (Kdk_48_17Aug11a_CC1) }\end{array}$ & 2 & 1 & $\mathrm{n} / \mathrm{a}$ & $\neq$ \\
\hline $\begin{array}{c}\text { Benchmark Ic2 } \\
\text { (Kdk_48_17Aug11a_CC2) }\end{array}$ & 2 & 2 & $\mathrm{n} / \mathrm{a}$ & $\ddagger$ \\
\hline $\begin{array}{c}\text { Benchmark Ic3 } \\
\text { (Kdk_48_17Aug11a_CC3) }\end{array}$ & 2 & 3 & 1700 & \\
\hline $\begin{array}{c}\text { Benchmark Ic4 } \\
\text { (Kdk_48_17Aug11a_CC4) }\end{array}$ & 2 & 4 & $\mathrm{n} / \mathrm{a}$ & b \\
\hline $\begin{array}{c}\text { 70\% Anisotropy Ic1 } \\
\text { (Kdk_48_16Jan12f_CC3) }\end{array}$ & 2 & 3 & $\begin{array}{c}1500 \\
900\end{array}$ & $\dagger$ \\
\hline $\begin{array}{c}\text { Block diagonal } W_{p, 4} \text { Ic1 } \\
\text { (Kdk_48_31Jan12d_CC3) }\end{array}$ & 2 & 3 & 2700 & \\
\hline $\begin{array}{c}\text { Block diagonal } W_{p, 4} \text { Ic2 } \\
\text { (Kdk_48_31Jan12d_CC4) }\end{array}$ & 2 & 4 & 2500 & \\
\hline
\end{tabular}

${ }^{\dagger}$ Different stability threshold in $x$ - and $y$ - directions.

\#Unable to achieve initially stable suspension.

${ }^{b}$ Controller achieves stable suspension of rotor, however, very sensitive to impacts. 
changing levels of CCS. To accomplish this, the Nc1 log decrement $\zeta_{1}$ was measured over a range of CCS magnitudes from zero up to the onset of instability. For a given controller and CCS magnitude, the mean log decrement was calculated by averaging the output of five decays measured at the four support bearing displacement sensors, i.e. a total of 20 samples. The stability maps in Figure 6.10 reveal the resulting trend and statistics. The standard deviations of the log decrement (as indicated by the error bars) tend to be larger at lower CCS values since the decay is much shorter given higher damping ratios. The maximum allowable CCS $Q_{\max }$ assuming optimum support damping was $3540 \mathrm{~N} / \mathrm{mm}$ and is shown as a dashed vertical line. From the data presented the following conclusions were drawn for the controllers designed without CCS uncertainty descriptions:

1. The controller designed with block-diagonal performance weight $W_{p, 4}$ extended the stability threshold by $30 \%$, the largest increase for controllers synthesized without a CCS uncertainty model.

2. The controller designed with $70 \%$ support stiffness anisotropy increased the stability threshold by only $18 \%$ from the benchmark. However, this controller had the largest effect on the $\mathrm{Nc} 1 \log$ dec in the absence of cross-coupling. At $\mathrm{Q}=0$, this controller achieved a $\log$ dec of 3.5 versus 2.2 for Benchmark I, a $59 \%$ increase.

3. Increased levels of stiffness anisotropy did not lead to improvements in the stability threshold. The 50\% support stiffness controller had different stability thresholds depending the direction of impulse excitation, $2300 \mathrm{~N} / \mathrm{mm}$ in the $x$-axis and 730 $\mathrm{N} / \mathrm{m}$ in the $y$-axis.

4. The full block diagonal $W_{p, 1}$ controller has a log decrement curve below the Benchmark I controller for all values of $Q$. The stability threshold of this controller was $33 \%$ lower than the benchmark.

With the inclusion of CCS uncertainty description the following conclusions were drawn for the controllers synthesized: 
1. Uncertainty descriptions CCS Model 1 and 2 produced controllers (Benchmark Ic1 and Ic2) that were unable to suspend the rotor. The coupling introduced between the different channels made the plant too difficult to stabilize with the given performance weights.

2. Benchmark Ic3 achieves a log decrement of 4.0 the highest log decrement among all controllers at $Q=0$. However, the stability threshold of the controller is $15 \%$ lower than Benchmark I.

3. The controllers designed with the block diagonal $W_{p, 4}$ performance weight achieve thresholds of $2500 \mathrm{~N} / \mathrm{mm}$ and $2700 \mathrm{~N} / \mathrm{mm}$, i.e., the third highest and highest, when using CCS Models 3 and 4, respectively. Surprisingly, CCS Model 3 has the higher threshold despite defining a smaller eigenvalue perturbation than CCS Model 4. Also, the log dec curve associated with CCS Model 4 remains below all the other controllers up to $1500 \mathrm{~N} / \mathrm{m}$.

In typical rotor-bearing systems, such as rotors supported by tilting-pad journal bearings, the damping ratio of the forward mode decreases with larger applied CCS, whilst the damping ratio of the backward mode increases Cloud (2007). In our experiments forward circular blocking has been used to selectively excite the forward mode. However, as shown in Figure 6.10b for the Block diagonal controller with Model CC3 and the Block diagonal controller with Model CC4, the damping ratio increases with the applied CCS up to a certain value of CCS, after which it decreases in the traditional manner. For both of these controllers the log decrement $\mathrm{Nc1}$ is maximized at a non-zero value of CCS in marked contrast to the other controllers. From a control theory standpoint, this observation is reconciled by recalling the local stability of closed-loop systems with unstable components (Stein, 2003). From a mechanical vibration standpoint, changes in the shape of the rotor whirl orbit at different levels of CCS have an effect on the rotor stability (Ehrich, 2004). 


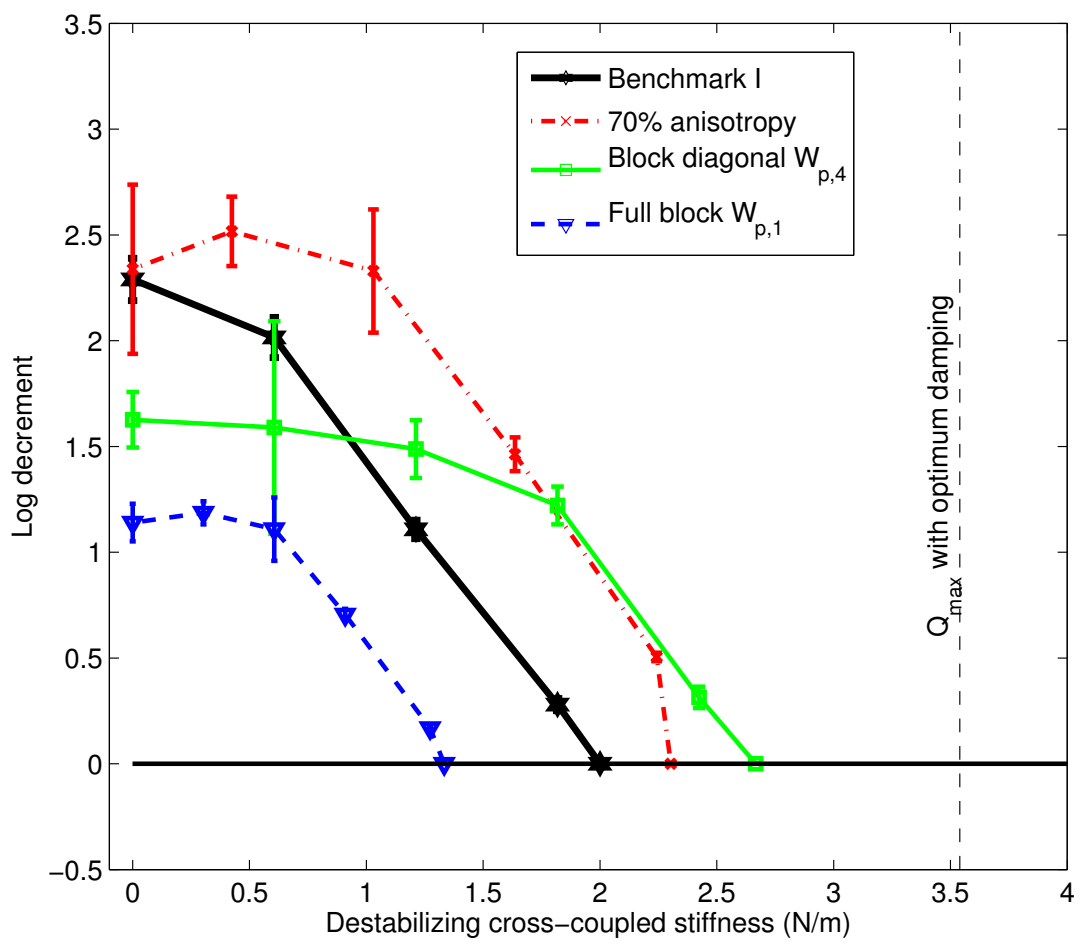

(a) Without CCS uncertainty model.

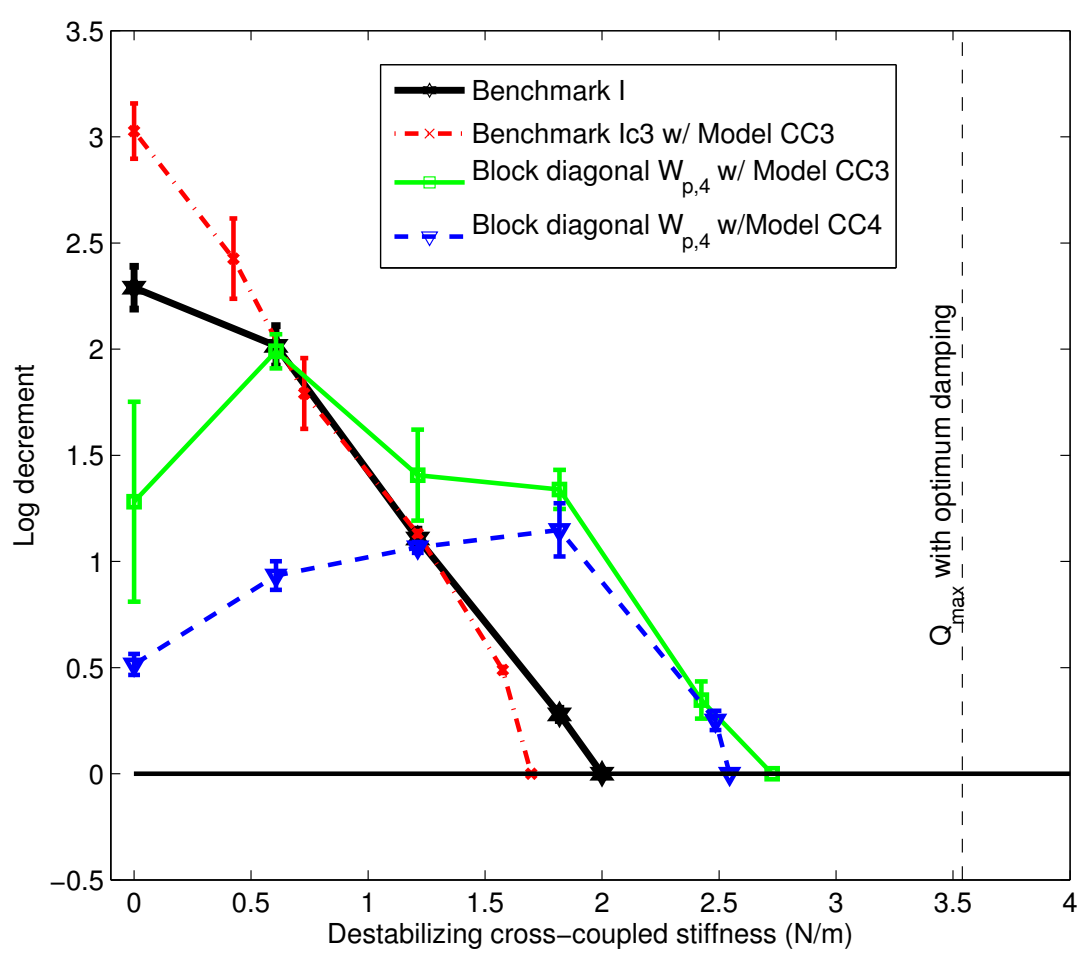

(b) With CCS uncertainty model.

Figure 6.10: Experimental stability sensitivity plot of log decrement of Nc1 versus destabilizing cross-coupled stiffness $Q$ for several controllers compared with the Benchmark I. 


\subsection{Discussions}

\subsubsection{Achievable versus Optimal Damping}

The idea of optimum support damping was introduced in Chapter 3, along with the maximum allowable destabilizing stiffness $Q_{\max }$. This theoretical upper limit on achievable destabilizing stiffness as a function of the mechanical properties of the rotor-bearing system, i.e., $K_{r}, M_{m}$ and $\omega_{c r}$ was calculated to be $3540 \mathrm{~N} / \mathrm{mm}$ in Chapter 4 . The Benchmark I controller demonstrated a stability threshold $56 \%$ of $Q_{\max }$, while the most capable controller demonstrated a stability threshold $76 \%$ of $Q_{\max }$. Two questions are how much further may the stability threshold be improved through better control design, and how reasonable is the theoretical optimal damping assumption to this application. Larsonneur (2009) pointed out that critical or very high damping of the rotor rigid body modes may be realized with AMBs without much difficulty. This fact was evident evident from our experimental stability analysis where log decrements of 4.0 (damping ratio 0.53 or $75 \%$ of critical damping) were measured for Nc1. Achieving high damping levels assumes the displacement sensors have a low noise floor, as the damping is realized by a derivative (or filtered derivative) action which leads to increased gain at high frequencies. Significant controller phase lead is straightforward to implement over the low frequencies around the rigid body modes.

To begin to understand the effect of the controller response on the stability threshold a comparison is made of Bode plots of the $x$-axis control channels (see Figure 6.11) of three controllers with differing stability thresholds: Benchmark I, Full block controller and Block diagonal $W_{p, 4}$ Ic3 with stability thresholds of $2000 \mathrm{~N} / \mathrm{mm}, 2700 \mathrm{~N} / \mathrm{mm}$ and $1300 \mathrm{~N} / \mathrm{mm}$, respectively. The $y$-axis channels is omitted as as the weighting functions are the same as the $x$-axis resulting in a similar response. In Figure 6.11a, the response from NDEX $\rightarrow$ NDEX, the magnitude and phase of the full block controller are less than magnitude and phase of the other controllers around Nc1, i.e., 45-55 Hz. This provides one 
reason why Nc1 was less damped for the full block controller, contributing to the lower stability threshold. Looking at Figure 6.11d, the response from DEX $\rightarrow$ DEX, the phase lead for the full block controller is comparable to the diagonal controller in the same frequency range. This revelation makes the estimation of the overall damping of $\mathrm{Nc} 1$ by evaluating the phase lead of the diagonal control channels less clear cut. The control responses along the off-diagonal control channels, i.e., DEX $\rightarrow$ NDEX and NDEX $\rightarrow$ DEX, are shown in Figure $6.11 \mathrm{~b}$ and $\mathrm{c}$. From the mode shape of $\mathrm{Nc1}$, we know displacements at both ends of the rotor are in phase. Therefore, a phase angle between 0 and $90^{\circ}$ will generate positive damping of a certain magnitude. From the remaining figures it is clear how the block diagonal $W_{p, 4}$ Ic3 controller has a larger magnitude response and positive phase angle indicating additional damping of Nc1. The SISO transfer function analysis of controller damping capabilities presented above is intuitive. However, this falls short of quantifying the overall damping produced by a MIMO controller with significant coupling between channels, and more importantly suggesting how far the effective damping at Nc1 may be increased with a more aggressive control law.

The analysis of the effect of unstable poles and the available bandwidth of the control system (see Section 3.2.2 on page 33 and Section 4.5.1 on page 88) sets an upper limit on the achievable minimum peak output sensitivity (Stein, 2003). Using the maximum singular value of the output sensitivity function, a generalization of this SISO result to multivariable systems is possible. The extent by which the available control bandwidth and hence achievable phase margin may potentially be increased through hardware modifications is revealed by searching for the weakest link among components in the feedback loop, i.e., power amplifiers, signal conditioning and data converting circuits, and delay associated with sampling and computation elements. The small signal bandwidth of the power amplifiers is a common limiting factor. Useful tricks include operating the amplifiers from a higher DC bus voltage (if supported), or tuning the output stage of the amplifier to better match the load impedance. 


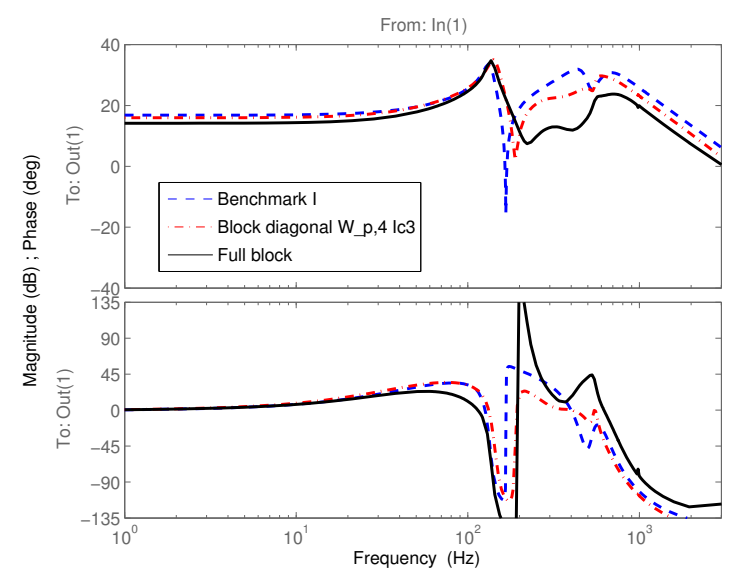

(a) $\mathrm{NDEX} \rightarrow \mathrm{NDEX}$

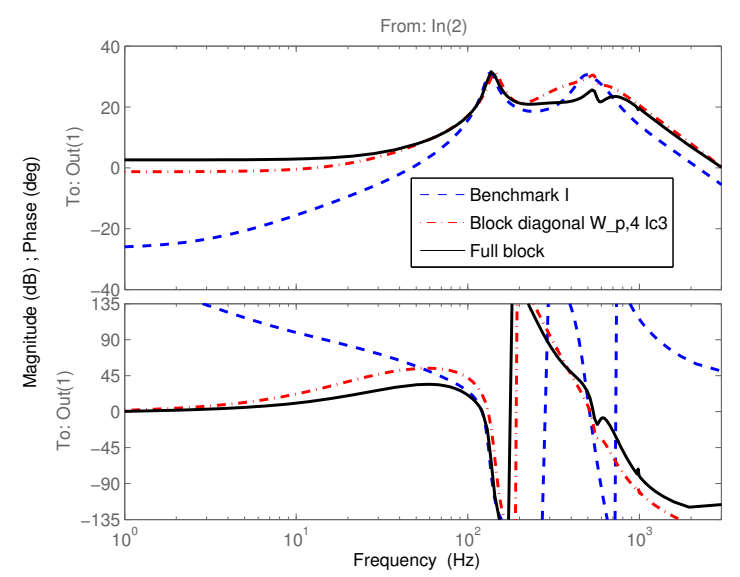

(c) NDEX $\rightarrow$ DEX

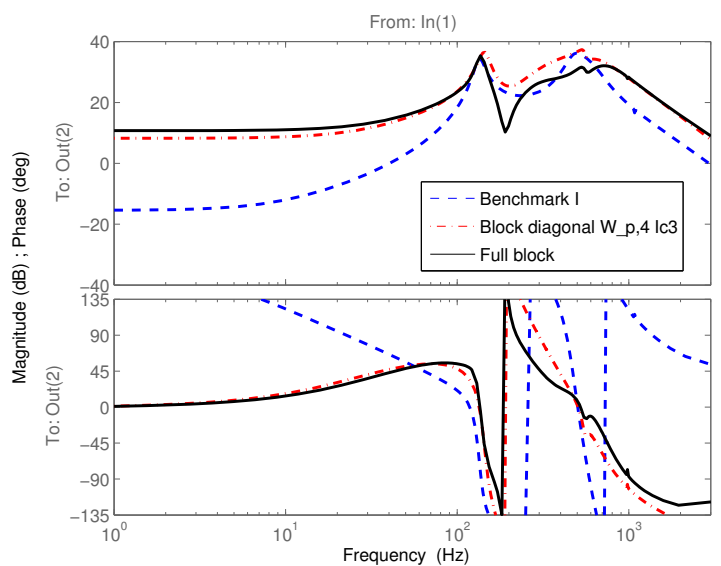

(b) $\mathrm{DEX} \rightarrow \mathrm{NDEX}$

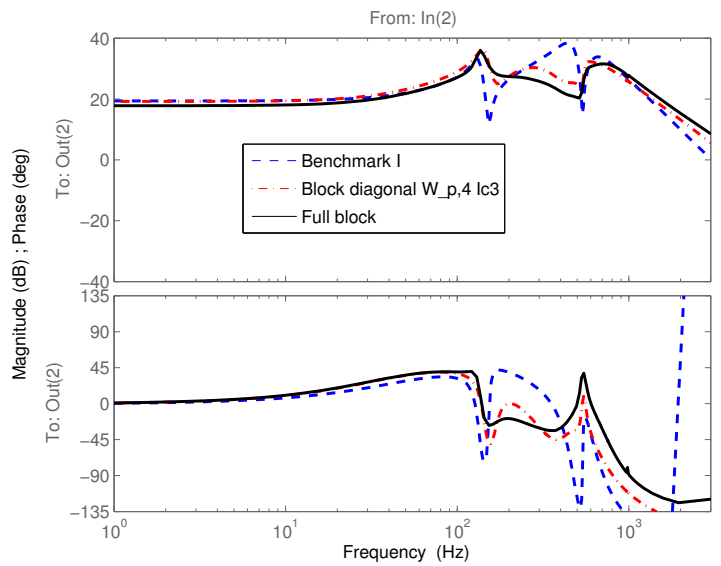

(d) DEX $\rightarrow$ DEX

Figure 6.11: Bode plots of three controllers comparing regions of positive phase. 
In answering the second question posed at the beginning of this section, we may consider how accurate the optimal damping result is. The optimal effective damping figure derived by Barrett (1979) made three key assumptions: actuator dynamics are absent, perfect colocation of actuators and sensors is achieved, and the presence of rigid bearing supports. The validity of each assumption is considered. The AMB actuators used in the MBTRI experimental setup have non-negligible dynamics in the form of a small-signal bandwidth and force slew rate limit, i.e., power bandwidth. The presence of these dynamics gives the actuator a non-ideal response and which manifests as phase lag or delay at higher frequencies. Though position sensors used to feedback the rotor displacement are not perfectly collocated with bearing center of action, they are close enough to be considered so. Furthermore, the modal displacement of $\mathrm{Nc} 1$ has very limited bending (see Figure 5.31 on page 147a and b) hence the shaft motion at sensor and actuator locations for a given AMB will always be in phase. Therefore, the perfect colocation assumption is valid. The bearing supports refer to the combination of the sensor mounts, the aluminium structure housing the AMB stator, the horizontal steel plate to which this housing is bolted, and the reinforced concrete block on which the steel plate is fastened. During earlier work on the MBTRI experiment, we noted that the aluminium housings and sensor mounts had several natural frequencies within the control bandwidth, i.e., they behaved as flexible damped supports instead of the rigid supports we expected. To minimize the compliance of the sensor mounts, a tuned mass damper in the form of a 1" steel nut was attached with putty to each sensor mount. To stiffen the aluminium housings, ribbed angle brackets were machined from structural steel and fastened to the steel base plate and one face of the NDE and DE housings. Though the changes forced many of the natural frequencies of the support beyond the controller bandwidth, some did remain and we could no longer consider the support to be rigid. The continued presence of damping and flexibility of the support subtracts from the effectiveness of a given level of bearing damping leading to a loss of optimality in the previously quoted optimal damping figure. 


\subsubsection{Performance versus Uncertainty}

Achieving a balance between the conflicting requirements of performance and robustness in face of plant model uncertainty is the central challenge of feedback control. This tradeoff is well visualized by the magnitude of the desired loop gain $\left|G_{\mathrm{d}}\right|$ shown in Figure 6.12. This plot generalizes several themes of control design in the frequency-domain presented in this dissertation (Sidi, 2001). Firstly, high open-loop gains contribute to strong disturbance rejection up to the open-loop unity gain crossover frequency $\omega_{\mathrm{c}}$. Model uncertainty below $\omega_{c}$ is required to be low to prevent the occurrence of instability arising from the high gain. At frequencies above $\omega_{c}$, controller gain roll-off produces a loop gain below unity which desensitizes the feedback loop to uncertainties in the model and measurement noise. While controllers to satisfy this trade-off for stable SISO plants are relatively straightforward to synthesize, significant challenges occur when attempting to apply the same classical loop shaping ideas to stabilize MIMO systems with RHP poles such as rotor-AMB systems (Skogestad \& Postlethwaite, 2005). The presence of multiple loop-gain crossovers from the bending modes of flexible rotors increase the difficulty in applying loop shaping control design strategies. An alternative and more versatile design approach that easily covers MIMO systems is the use of closed-loop transfer functions, e.g., system sensitivity functions, to define the optimal performance and robustness trade-off. The worst case upper bound on the magnitudes of system sensitivity functions, i.e., maximum singular values the output sensitivity $\bar{\sigma}(S)$, complementary output sensitivity $\bar{\sigma}(T)$, control sensitivity $\bar{\sigma}(K S)$ and process sensitivity $\bar{\sigma}(G S)$, have been used to enforce performance criteria for the MBTRI experiment (see Section 5.4 on page 112). It was shown in Section 6.1.2 on page 152 that these bounds were satisfied for the nominal operating point of the experiment.

The dynamics of rotor-bearing system are known to vary significantly with changes in the rotation speed and the magnitude of CCS excitation. Several uncertainty models were developed in Section 5.3 on page 103 to capture these variations in a form suitable for robust control design using $\mu$-synthesis. Two powerful tools namely the $\nu$-gap metric and 


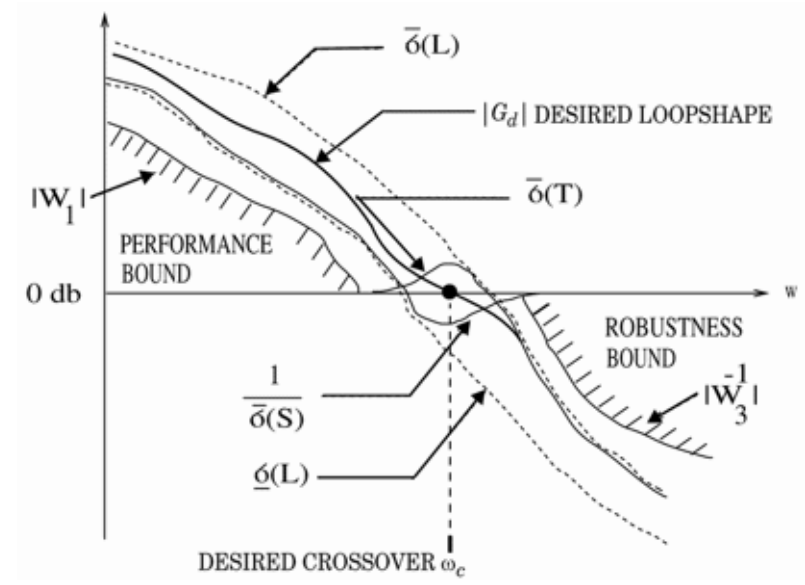

Figure 6.12: Loop gain specifications for performance and robustness trade off (The Mathworks, 2009).

$\mu$-analysis were used to study the uncertainty model sets and compare robust performance trade offs. Such analysis is crucial to help develop insights into whether to control results already presented may be improved.

The $v$-gap metric analysis has shown that capturing the CCS uncertainty using a phenomenological approach has an overwhelming advantage over direct modeling. CCS Model $l$ and 2 both had gap metrics of unity, indicating that robust stabilization of models based on either description could not be possible using a controller that stabilized the nominal plant. In retrospect it was not surprising that CCS Models 3 and 4 had similar gap metrics. The nominal values of the uncertain Nc1 eigenvalue parameter was $220 \mathrm{rad} / \mathrm{s}$ and $230 \mathrm{rad} / \mathrm{s}$, respectively. The only difference between the two descriptions was that CCS Model 4 had an eigenvalue range that extended $10 \mathrm{rad} / \mathrm{s}$ above and below that of CCS Model 3. This resulted in a slight increase in the $\mu$-upper bound from 1.32 to 1.34 for the block diagonal $W_{p, 4}$ performance weight. However, for the benchmark performance weights, the $\mu$-upper bound decreased from 1.17 to 1.16 as CCS Model 3 was replaced with CCS Model 4. In all cases, controllers developed with CCS Model 4 had a lower stability threshold than controllers designed with CCS Model 3, demonstrating its conservatism. CCS Model 4 may be improved by trying to better identify a nominal model that in a given uncertainty set defined by an eigenvalue perturbation. The optimal nominal model would be that which 


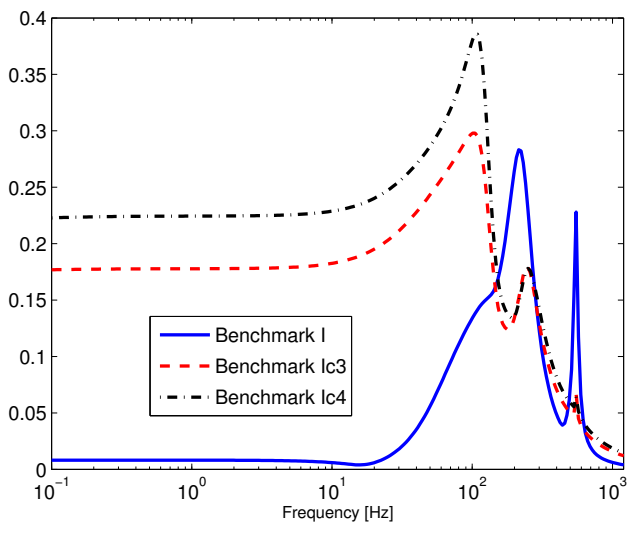

(a) Benchmark controller weights

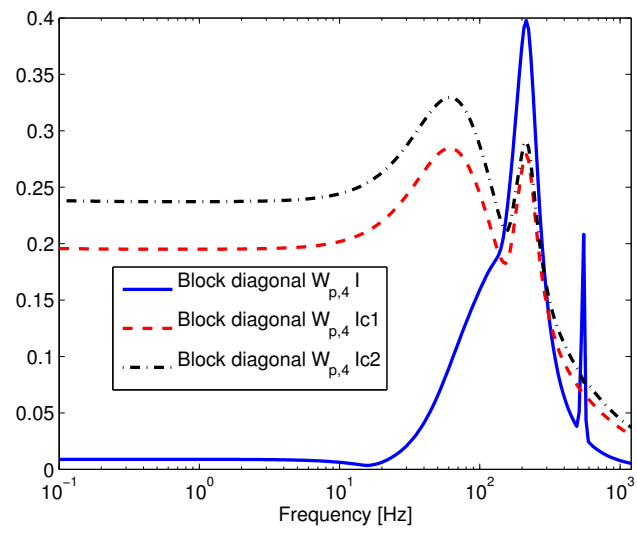

(b) Block diagonal $W_{p, 4}$ weights

Figure 6.13: Maximum singular value of interaction between model uncertainty and weighted performance outputs, $\left\|T_{z v}(j \omega)\right\|_{\infty}$ or $\bar{\sigma}\left(M_{21}(j \omega)\right)$.

has a minimum gap metric to all others (Jafarian \& Haggblom, 2010).

$\mu$-analysis of the $M-\Delta$ feedback interconnection was performed in Chapter 5 to analyze the RS, NP and RP measures to determine which are driving the problem (Honeywell \& Lockheed-Martin, 1996). It was observed across all the controllers that satisfying the NP objective, particularly below $100 \mathrm{~Hz}$, was the limiting factor. Further decomposing the problem revealed that the specification on the system gain from exogenous disturbances to the weighted position error had the largest contribution to the NP objective. The system interaction between the model uncertainties and weighted performance outputs for three different model uncertainty sets, i.e., Speed Model 2 alone, Speed Model 2 + CCS Model 3, and Speed Model $2+$ CCS Model 4 are shown in the form of maximum singular values of $M_{21}$ in Figure 6.13. In Figure 6.13a the benchmark weighting functions are used, while in Figure $6.13 \mathrm{~b}$ the block diagonal $W_{p, 4}$ weights are used. The peak value for all combinations is less than 0.4 . Therefore, neither stability robustness nor uncertain performance seem to be limiting, rather the nominal performance specification $\bar{\sigma}\left(M_{22}(j \omega)\right)$ enforced by $W_{p}$ and $W_{r}$. To improve the worst case $\mu$-bounds, relaxing the performance criteria with changes to $W_{p}$ or $W_{r}$ would have the most benefit. 


\section{Chapter 7}

\section{Conclusions and Recommendations}

\subsection{Summary of Accomplishments}

This dissertation presents the results of an investigation into rotordynamic instability in a high speed rotor-AMB test rig with similitude to a supercritical industrial compressor. The occurrence of rotordynamic instability in centrifugal compressors arising from fluidstructure interactions at the seal and impeller locations may, at best, limit machine throughput, and at worst, result in machine damage due to its sudden and violent onset. This industrially motivated problem lends itself well to the application of control theory. In particular, we have investigated the use of robust control theory to extend the stability threshold. The following accomplishments are noted:

- A high speed rotor-AMB test rig has been designed, constructed and commissioned for rotordynamic stability investigations. In addition to the standard pair of radial magnetic bearings necessary to support the rotor, the novel rotor design accommodated an additional pair of radial magnetic bearings located at the midspan and quarterspan of the rotor. The midspan bearing generated the dynamic load characteristic of the cross-coupled stiffness mechanism that leads to instability in industrial machines. While the quarterspan bearing provided arbitrary excitations to use during 
the evaluation of rotor-bearing system stability. Beyond the scope of this study, the MBTRI hardware also provides a challenging yet practical platform for the implementation of a wide range of control theories under development at the University.

- A reduced-order nominal model of the plant dynamics has been developed from first principles and validated using online system identification techniques.

- Several uncertainty models representing the effects of operating speed variation and destabilizing cross-coupled stiffness (CCS) magnitude variation have been developed and used to augment the nominal plant dynamics.

- $v$-gap metric analysis of the nominal and uncertain plant models revealed the effects of the uncertainty modeling technique on the overall conservatism of the system model. Less conservative models are preferred for the synthesis of model-based controllers.

- Frequency domain weighting functions have been developed to translate the engineering specifications of stability and robustness into cost functions to be optimized by the controller synthesis procedure.

- Several robust $\mu$-synthesis controllers have been designed to safely suspend the rotor as it is operated from 0 to $18,000 \mathrm{rpm}$, crossing one bending natural frequency.

- Using experimental damping ratio measurement techniques, the stability sensitivity as a function of the destabilizing force was determined. With the highest performing controller, the stability threshold was demonstrated to be $36 \%$ higher than initial benchmark value. This brings us to within $76 \%$ of the maximum allowable instability $Q_{\max }$ assuming the presence of optimum damping. Limitations of the optimum damping and $Q_{\max }$ figure for rotor-AMB systems were highlighted.

- Extensive robustness analysis using the structured singular value framework revealed that nominal performance specifications, specifically criteria governing the system 
gain from finite energy disturbance inputs to weighted position errors are most critical to reducing the $\mu$-bound.

There is an emerging view that control system technology cannot be confined to developing algorithms to close feedback loops from sensors to the actuators, but can also play a role in shaping the engineering design of the overall system to be controlled (van der Schaft \& Maschke, 2009). This is particularly true for complex mechatronic products with coupled multivariable dynamics in which the controller plays a critical role in achieving the performance specifications. A brief survey of such applications (apart from rotor-AMB systems) that have applied robust control techniques similar to those described in this dissertation include the track-following subsystem of an optical disc reader (Steinbuch et al., 1998), nanometer precision motion control for a lithography wafer stage (van de Wal et al., 2002), servo control of a high-friction metering valve for gas turbine engines (Shahroudi \& Young, 2008), and shape control of deformable mirrors using magnetic fluids (Iqbal et al., 2010). These diverse examples exhibit several commonalities, one of which is the trade-off between uncertainty and performance, and the other is the central role modeling plays in the process of control design. A firm grasp of the fundamental limitations on achievable performance with feedback that arise from the mechanics of the system are crucial as the demand for high performance control systems continues. The engineering investment in model-based tools such as structured singular value $\mu$ for synthesis and analysis provides significant insight into the effects and feasibility of various specifications for the final design.

\subsection{Recommendations for Further Work}

Several recommendations for further work on the MBTRI experiment have been motivated by the controller analysis experience and experimental results. First, we conclude that the performance specifications defined using the frequency domain weighting functions were a 
critical limitation to improving the $\mu$-bounds. The initial design of the weighting functions and subsequent iteration to improve their end result, as described in Chapter 5, was a manual heuristic process. Exploring a means of automating the optimization of performance weighting functions can lead not only to large time savings during the design process and better performance weights. Lanzon (2000) proposed an algorithm that modifies D-K iteration in order to maximize the performance weights with respect to some cost function while simultaneously minimizing the $\mu$-upper bound. This approach promises serious dividends when coupled (non-diagonal) performance weights are desired. However, the complexity of the algorithm implementation and somewhat lack of transparency has hitherto prevented its wider adoption. Second, additional insight to the physical eigenvalue perturbations which result from CCS may be possible through experimental system identification techniques applied to the rotor-AMB system under various magnitudes of destabilizing forces. The information from the system identification procedures would be vitally important in performing model (in)validation. Data-based uncertainty modeling techniques can be used to reconcile differences with the first principles approach used in the dissertation. (Haggblom, 2007, 2010) describe using convex optimization techniques to minimize the gap metric of model uncertainty sets so as to obtain an optimal nominal model in the v-gap metric sense. Third, the author also concludes that some assumptions critical to the optimal damping derivation may be invalid for rotor-AMB systems, in general. Therefore, the author suggests that optimal damping be derived for a multivariable rotor-AMB system taking into account the actuator dynamics, i.e., small-signal and power bandwidth. Furthermore, since the rotor mode ( $\mathrm{Nc1}$ ) excited by the instability exhibits less bending than witnessed in practical supercritical compressors, a comparative derivation based on rigid rotor model is warranted. It is likely these new values of optimal damping will have a lower maximum allowable instability threshold than the value derived by Barrett (1979). Additional aspects of rotor-AMB systems that may also be taken into account in a new derivation may include sensor/actuator (non)colocation, and the presence of damped, flexi- 
ble supports. Fourth, motivated by recent literature on the use of tip-clearance actuation to extend the stability margins of high speed compressors experiencing aerodynamic stall and surge (Spakovszky et al., 2000; Yoon et al., 2010), the author is curious as to what effect a similar rotor position modulation control strategy could have on the stability threshold with respect to rotordynamic instability. There is one major difference between the classes of two problems, the latter involves unstable rotor motion, while the former involves unstable fluid motion around the rotor. A comparative investigation into the use of stochastic or robust disturbance estimators/observers to extend the stability threshold and how this compares with the $\mu$-synthesis approach used in this dissertation is also recommended for future work. 


\section{Publications}

1. "Design, construction and modeling of a flexible rotor active magnetic bearing test rig,” Mushi, S.E., Lin, Z., Allaire, P.E., IEEE/ASME Trans. Mechatronics, 2011, Vol. PP (99), DOI: 10.1109/TMECH.2011.2160456.

2. "Robust control design for a supercritical rotor supported by active magnetic bearings,” Mushi, S.E., Lin, Z., Allaire, P.E., (in preparation).

3. "Stability analysis of a flexible rotor on active magnetic bearings subject to aerodynamic loads," Mushi, S.E., Lin, Z., Allaire, P.E., in Proc. 12th Int. Symp. Mag. Brgs. (ISMB 12), Wuhan, China, 22-25 August 2010.

4. "Design, construction and modeling of a flexible rotor active magnetic bearing test rig," Mushi, S.E., Lin, Z., Allaire, P.E., in Proc. ASME Turbo Expo, Glasgow, Scotland, $14-18$ June 2010.

5. "Aerodynamic cross-coupling in a flexible rotor on magnetic bearings: control design and implementation,” Mushi, S.E., Lin, Z., Allaire, P.E., S.M. Evans, in Proc. 11th Int. Symp. Mag. Brgs. (ISMB 11), Nara, Japan, 26 - 29 August 2008. 


\section{References}

Abzug, M.J., \& Larrabee, E.E. 2002. Airplane Stability and Control: A History of the Technologies That Made Aviation Possible. Cambridge.

ACKermann, J. 1993. Robust Control: Systems with Uncertain Physical Parameters. Springer-Verlag.

Ahrens, M., Frei-Spreiter, B., \& Wieser, R. 2000. Cost efficient electric highspeed drives with magnetic bearings for the COG market. Pages 507-512 of: Proc. $7^{\text {th }}$ Int. Symp. Magn. Brgs. ISMB7.

Alford, J.S. 1965. Protecting turbomachinery from self-excited rotor whirl. ASME J. Eng. for Power, 87(4), 333-344.

Allaire, P.E., Imlach, J., McDonald, P., Humphris, R., Lewis, D.R., BanerJeE B., Blair, B.J., Claydon J., \& FlaCk, R.D. 1989. Design, construction and test of magnetic bearings in an industrial canned motor pump. In: Proc. $6^{\text {th }}$ Intl. Pump Users Symp.

Allaire, P.E., Maslen, E.H., Humphris, R.R., Knospe, C.R., \& Lewis, D.W. 1994. CRC Handbook of Lubrication: Theory and Practice of Tribology. 2 edn. Vol. 3. CRC Press. Chap. Magnetic Bearings, pages 577-600.

Allaire, P.E., Maslen, E.H., Kim, H.C., Bearnson, G.B., \& Olsen, D.B. 1996. Design of a magnetic bearing-supported prototype centrifugal artificial heart pump. STLE Trib. Trans., 39(3), 663-669.

Antila, M. 1998. Electromechanical Properties of Radial Active Magnetic Bearings. Ph.D. thesis, Helsinki University of Technology.

API 617. 2002. Axial and Centrifugal Compressors and Turboexpanders for Petroleum, Chemical and Gas Industry Services. 
API 684. 2005. Standard Paragraphs Rotordynamic Tutorial: Lateral Critical Speeds, Unbalance Response, Stability, Train Torsionals, and Rotor Balancing.

Astrom, K.J., \& Wittenmark, B. 1997. Computer-controlled Systems: Theory and Design. 3 edn. Upper Saddle River, NJ, USA: Prentice-Hall, Inc.

BALAS, G. 1990. Robust Control of Flexible structures: Theory and Experiments. Ph.D. Dissertation, California Institute of Technology.

Balas, G., \& Young, P. 1995. Control design for variations in structural natural frequencies. AIAA J. Guid., Control and Dyn., 18(2), 325-332.

BAlas, G., \& Young, P.M. 1999. Sensor selection via closed-loop control objectives. IEEE Trans. Contr. Syst. Technol., 7(6), 692-705.

BALAS, G.J., \& DoYLE, J.C. 1994. Robustness and performance trade-offs in control design for flexible structures. IEEE Trans. Contr. Syst. Technol., 2(4), 352-361.

BARRETT, L. 1979. Stability and Nonlinear Response of Rotor-bearing Systems with Squeeze Film Bearings. Ph.D. thesis, University of Virginia.

Barrett, L.E., Gunter, E.J., \& Allaire, P.E. 1978. Optimum bearing damping and support damping for unbalance response and stability of rotating machinery. ASME J. Engr. for Power, 100, 89-94.

Bates, D., \& Postlethwaite, I. 2002. Robust Multivariable Control of Aerospace Systems. Delft University Press Science.

BAUMANN, U. 1999. Rotordynamic stability tests on high-pressure radial compressors. In: Proc. of 28th Turbomachinery Symp.

BIDAUT, Y., \& BAUMANN, U. 2010. Improving the design of a high pressure casing with the help of finite element analysis to ensure the rotordynamic stability of a high pressure centrifugal compressor equipped with a hole pattern seal. In: Proc. ASME Turbo Expo GT2010.

BLACK, H.F. 1976. The stabilizing capacity of bearings for flexible rotors with hysteresis. ASME J. Engr. for Ind., 98(1), 87-91.

Bleuler, H., Gahler, C., Herzog, R., Larsonneur, R., Mizuno, T., Siegwart, R., \& WoO, SHAO-JU. 1994. Application of digital signal processors for industrial magnetic bearings. IEEE Trans. Contr. Syst. Technol., 2(4), 280-289. 
Bode, H. 1945. Network Analysis and Feedback Amplifier Design. D. Van Nostrand Company.

Boerlage, M. 2008. Rejection of Disturbances in Multivariable Motion Systems. Ph.D. Dissertation, Eindhoven University of Tehnology.

Bornstein, K. 1991. Dynamic load capabilities of active electromagnetic bearings. ASME J. Trib., 113(3), 598-603.

Boyd, S.P., El Ghaoui, L., Feron, E., \& Balakrishnan, V. 1994. Linear Matrix Inequalities in Systems and Control Theory. SIAM.

BraAtz, R.D., Young, P.M., Doyle, J.C., \& Morari, M. 1993. Computational complexity of mu calculation. Pages 1682-1683 of: American Control Conference.

Brown, G.V., Kascak, A.F., Jansen, R.H., Dever, T.P., \& Duffy, K.P. 2005. Stabilizing Gyroscopic Modes in Magnetic Bearing-Supported Flywheels by Using CrossAxis Proportional Gains. In: AIAA GNC Conference and Exhibit.

Brown, R.N. 2005. Compressors: Selection and Sizing. Oxford University Press.

BuCHER, C. 1985. Contribution to the Modeling of Flexible Structures for Vibration Control. Ph.D. Dissertation, Swiss Federal Institute of Technology.

Chaudhry, J., \& Sheth, P.N. 2008. Rotor Dynamic Analysis in Matlab Framework. Tech. rept. 530 (ROMAC); UVA/643092/MAE08 (UVA). ROMAC Laboratory.

Chen, C.-T. 1998. Linear System Theory and Design (Oxford Series in Electrical and Computer Engineering). Third. edn. Oxford Univ Pr.

Chen, M., \& Knospe, C. 2007. Control approaches to the suppression of machining chatter using active magnetic bearings. IEEE Trans. Contr. Syst. Technol., 15(2), 220232.

Chilali, M., Gahinet, P., \& Apkarian, P. 1999. Robust pole placement in LMI regions. IEEE. Trans. Autom. Contr., 44(12), 2257-70.

ChILds, D.W. 1993. Turbomachinery Rotordynamics: Phenomena, Modeling, and Analysis. Wiley.

Cloud, C.H., Maslen, E.H., \& BARrett, L.E. 2009. Damping ratio estimation techniques for rotordynamic stability measurments. ASME J. Eng. Gas Turb. Power, 131. 
Cloud, H. 2007. Stability of Rotors Supported by Tilting Pad Journal Bearings. Ph.D. Dissertation, University of Virginia.

CRIQUi, A.F., \& WEndT, P.G. 1980. Design and closed loop testing of high-pressure centrifugal gas compressors for the suppression of subsynchronous vibration. ASME J. Engr. for Power, 102, 136-140.

Dimond, T. 2011. A review of tilting pad bearing theory. Int. J. Rotat. Machinery, 2011, 23.

Doyle, J., Packard, A., \& Zhou, K. 1991. Review of LFTs, LMIs, and $\mu$. Pages 1227-1232 of: Proc. 30th IEEE Conf. Dec. and Contr., vol. 2.

DoYle, J.C. 1982. Analysis of feedback systems with structured uncertainty. IEE Proc. Part D, 129, 242-250.

Doyle, J.C., Glover, K., Khargonekar, P., \& Francis, B. 1989. State space solutions to standard $\mathrm{H} 2$ and $\mathrm{H}$-infinity control problems. IEEE Trans. Autom. Control, 34, 831-847.

Doyle, J.C., Francis, B., \& Tannenbaum, A. 1990. Feedback Control Theory. Macmillan Publishing Company.

EHRICH, F., \& CHILDS, D. 1984. Self-excited vibration in high-performance turbomachinery. Mechanical Engineering, May, 66-79.

EHRICH, F. F. 2004. Handbook of Rotordynamics. Krieger Publishing Company.

EL-SAKKARY, A.K. 1985. The gap metric: Robustness of stabilization of feedback systems. IEEE Trans. Autom. Control, 30(3), 240-247.

Elliott. 2010. Multi-Stage Centrifugal Compressors Product Brochure. http: //www . elliott-turbo.com/Files/Admin/Literature/compressors.pdf. Accessed Feb. 2010.

Englehart, M.J., \& SMith, M.C. 1990. A four-block problem for H infin; design: properties and applications. Pages 2401-2406 of: Proc. 29th IEEE Conf. Decision Control, vol. 4.

Fittro, R. 1998. A High Speed Machining Spindle with Active Magnetic Bearings: Control Theory, Design and Application. Ph.D. Dissertation, University of Virginia. 
FitTRO, R.L., \& KNOSPE, C.R. 2002. Rotor compliance minimization via mu-control of active magnetic bearings. IEEE Trans. Contr. Syst. Technol., 10(2), 238-249.

FozI, A.A. 1987. An Examination of Gas Compressor Stability and Rotating Stall. Tech. rept. N87-22201. NASA.

FrANCHEK, M.A. 1996. Selecting the performance weights for the mu and H-infinity synthesis methods for SISO regulating systems. ASME J. Dyn. Sys. Meas. Contr., 118, 126-131.

Franklin, G.F., Powell, J.D., \& Emani-Naeini, A. 1994. Feedback Control of Dynamic Systems. Addison Wesley.

FreUdenberG, J.S., \& LoOze, D.P. 1985. Right half plane poles and zeros and design tradeoffs in feedback systems. IEEE Trans. Autom. Control, 30(6), 555-565.

Gahler, C., Mohler, M., \& Herzog, R. 1997. Multivariable identification of active magnetic bearing systems. JSME Intl. J. Series C, 40(4), 584-592.

GARG, S. 2008. Implementation Challenges for Multivariable Control: What You Did Not Learn in School! Tech. rept. NASA/TM-2008-215027. NASA Glenn Research Center.

Goodwin, G., Graebe, S., \& Salgado, M. 2000. Control System Design. Prentice Hall.

Green, M., \& Limebeer, D.J.N. 1995. Linear Robust Control. Upper Saddle River, NJ, USA: Prentice-Hall, Inc.

Gu, D.-W., Petkov, P.Hr., \& Konstantinov, M.M. 2005. Robust Control Design with MATLAB. Secaucus, NJ, USA: Springer-Verlag.

Habermann, H., \& Brunet, M. 1984. The active magnetic bearing enables optimum damping of flexible rotor. ASME Paper 84-GT-117.

HagGblom, K.E. 2007. Data-based modeling of block-diagonal uncertainty by convex optimization. Pages 4637-4642 of: Proceedings of American Control Conference.

HaGGBlOM, K.E. 2010. Convex formulations for data-based uncertainty minimization of linear uncertainty models. Pages 501-505 of: Proceedings Eleventh International Conference on Control, Automation, Robotics and Vision. 
Hetherington, H.L., Kraus, R.F., \& Darlow, M.S. 1990. Demonstration fo a supercritical composite helicopter power transmission shaft. J. Am. Helicopter Soc., 35(1), 23-28.

Hirschmanner, M., Steinschaden, N., \& Springer, H. 2002. Adaptive control of a rotor excited by destabilizing cross-coupling forces. Pages 38-45 of: Proc. $6^{\text {th }}$ IFToMM Int. Conf. Rotor Dynamics.

Holopainen, T.P. 2004. Electromechanical Interaction in Rotordynamics of Cage Induction Motors. Ph.D. thesis, Helsinki University of Technology.

HONEyWELl, \& LOCKHEED-MARTIN. 1996. Application of multivariable control theory to aircraft control laws; Final Report : Multivariable control design guidelines. Tech. rept. WL-TR-96-3099. Wright Laboratory, US Airforce Materiel Command.

HuAng, B. 2007. Optimum Balancing of High Speed Uncertain Flexible Rotor Systems Using Convex Optimization. Ph.D. Dissertation, University of Virginia.

InMAn, D. 2006. Vibration with Control. Wiley.

Iqbal, A., Wu, Zhizheng, \& Ben Amara, F. 2010. Mixed-sensitivity $H_{\infty}$ control of magnetic-fluid-deformable mirrors. IEEE/ASME Trans. Mech., 15(4), 548-556.

ISO 14839-2. 2004. Mechanical vibration - Vibration of rotating machinery equipped with active magnetic bearings - Part 2: Evaluation of vibration.

ISO 14839-3. 2006. Mechanical vibration - Vibration of rotating machinery equipped with active magnetic bearings - Part 3: Evaluation of stability margin.

JAFARIAN, S.H., \& HagGBlOM, K.E. 2010. Identification of a nominal model in the nu-gap metric. Tech. rept. 10-1. Abo Akademi University, Abo, FInland.

Kasarda, M. E. F., Allaire, P. E., Norris, P. M., Mastrangelo, C., \& Maslen, E. H. 1999. Experimentally Determined Rotor Power Losses in Homopolar and Heteropolar Magnetic Bearings. ASME J. Eng. Gas. Turb. Power, 121(4), 697-702.

Khalil, H. 2001. Nonlinear Systems. 3 edn. Prentice Hall.

Knospe, C., \& Maslen, E.H. 1997. Meaningful control experiments. Pages 2703 2707 of: Proc. American Control Conf., vol. 5. 
Knospe, C.R., Hope, R.W., Fedigan, S.J., \& Williams, R.D. 1995. Experiments in the control of unbalance response using magnetic bearings. Mechatronics, 5(4), 385400.

KNOSPE, C.R., TAMER, S.M., \& FITTRO, R. 1997. Rotor synchronous response control: approaches for addressing speed dependence. J. Vib. Contr., 3, 435-458.

Kocur, J.A., Nicholas, J.C., \& LEE, C.C. 2007. Surverying tilting pad journal bearing and gas labyrinth seal coefficients and their effect on rotor stability. Pages 1-10 of: Proc. $37^{\text {th }}$ Turbomach. Symp.

KumARESAN, R., \& TUFTS, D. 1982. Estimating the parameters of exponentially damped sinusoids and pole-zero modeling in noise. IEEE Trans. Acoust. Spch. Signal Proc., 30(6), 833-840.

KwAnKA, K. 2000. Dynamic coefficients of stepped labyrinth gas seals. ASME J. Eng. Gas. Turb. Power, 122(3), 473-477.

LANG, O., WASSERmann, J., \& Springer, H. 1996. Adaptive vibration control of a rigid rotor supported by active magnetic bearings. ASME J. Eng. Gas Turb. Power, 118(4), 825-829.

Lanzon, A. 2000. Weight Selection in Robust Control: An Optimization Approach. Ph.D. Dissertation, University of Cambridge.

Larsonneur, R. 2009. Magnetic Bearings: Theory, Design, and Application to Rotating Machinery. Springer. Chap. 2: Principle of Active Magnetic Suspension, pages 27-68.

LI, G. 2006. Robust Stabilization of Rotor-Active Magnetic Bearing Systems. Ph.D. Dissertation, University of Virginia.

LJUNG, L. 1998. System Identification: Theory for the User. 2 edn. Prentice Hall PTR.

LOsCH, F. 2002. Identification and Automated Controller Design for Active Magnetic Bearing Systems. Ph.D. thesis, ETH Zurich.

Losch, F., Gahler, C., \& Herzog, R. 1998. Mu-Synthesis controller design for a 3MW pump running in AMBs. Pages 415-428 of: Proc. 6th Int. Symp. Magn. Bearings.

LUND, J.W. 1974. Stability and damped critical speeds of a flexible rotor in fluid-film bearings. ASME J. Eng. Ind., 96(2), 509-517. 
LUND, J.W. 1975. Some unstable whirl phenomena in rotating machinery. Shock and Vib. Digest, 7(6), 5-12.

Mackenroth, U. 2004. Robust Control Systems: Theory and Case Studies. SpringerVerlag.

MASLEN, E.H., \& BIELK, J.R. 1992. A stability model for flexible rotors with magnetic bearings. ASME J. Dyn. Sys. Meas. Contr., 114, 172-175.

MAslen, E.H., \& SAWICKI, J. T. 2007. Mu-Synthesis for magnetic bearings: why use such a complicated tool? Pages 1103-1112 of: Proc. ASME Int. Mech. Engr. Congr. Expo IMECE2007.

Maslen, E.H., Hermann, P., Scott, M., \& Humphris, R.R. 1988. Practical limits to the performance of magnetic bearings: peak force, slew rate, and displacement sensitivity. In: Proc. NASA Conf. Magn. Susp. Tech., 2-4 Feb.

Maslen, E.H., Barrett, L., Allaire, P.E., Brockett, T., Knospe, C., SorTORe, C., Rockwell, R., \& Kasarda, M. 1996. Magnetic Bearing Performance Audit. Tech. rept. 395 (ROMAC); UVA/643092/MAE96/495 (UVA). ROMAC Laboratory, University of Virginia.

Maslen, E.H., KNOSPe, C.R., \& ZHU, L. 2006. An enhanced dynamic model for the actuator/amplifier pair in AMB systems. Pages 395-399 of: Proc. 10th Int. Symp. Magn. Bearings, 21-23 August 2006.

MeEKer, D.C. 2009. Finite Element Method Magnetics Vesion 4.2. www. femm.info. Build 02Nov2009.

Meeker, D.C., Maslen, E.H., \& Noh, D.M. 1995. A wide bandwidth model for the electrical impedance of magnetic bearings. In: Proc. 3rd Intl. Symp. Magn. Susp. Techn.

Mitchell, D.M. 2001. Tricks of the Trade: Understanding the right-half-plane zero in small-sgnal DC-DC converter models. IEEE Power Electronics Society, January, 5-6.

Moore, J. J., Walker, S.R., \& KuzDZAL, M.J. 2002. Rotordynamic stability measurement during full-load full-presssure testing of a 6000 psi re-injection centrifugal compressor. Pages 29-38 of: Proc. $31^{\text {th }}$ Turbomach. Symp.

Moore, J.J., \& RANSOM, D.L. 2010. Centrifugal compressor stability prediction using a new physics based approach. ASME J. Eng. Gas. Turb. Power, 132(8), 082402. 
Moore, J.J., Camatti, M., Smalley, A.J., Vannini, G., \& Vermin, L.L. 2006. Investigation of a rotordynamic instability in a high pressure centrifugal compressor due to damper seal convergence. In: Proc. $7^{\text {th }}$ IFToMM Conf. on Rotor Dyn.

Mushi, S.E. 2008. An Active Magnetic Bearing Test Rig for Aerodynamic CrossCoupling: Control Design and Implementation. M.Phil. thesis, University of Virginia.

Mushi, S.E., Lin, Z., \& Allaire, P.E. 2010. Design, construction and modeling of a flexible rotor active magnetic bearing Test Rig. Pages 443-452 of: Proc. ASME Turbo Expo.

Mushi, S.E., Lin, Z., \& Allaire, P.E. 2011. Design, construction and modeling of a flexible rotor active magnetic bearing test rig. IEEE/ASME Trans. Mechatronics., PP(99). (to appear).

NoH, D. 1996. Self-sensing Magnetic Bearings Driven by a Switching Power Amplifier. Ph.D. thesis, University of Virginia.

Nordmann, R. 2009. Magnetic Bearings: Theory, Design, and Application to Rotating Machinery. Springer. Chap. 11 : Identification, pages 299-318.

Ogunnaike, B.A. 1996. A contemporary industrial perspective on process control theory and practice. Ann. Rev. Control, 20, 1-8.

PACKARD, A., \& Doyle, J. 1993. The complex structured singular value. Automatica, 29(1), 71-109.

PAidoussis, M.P., PRICE, S.J., \& DE LANGRe, E. 2011. Fluid-structure Interactions: Cross-flow-induced Instabilities. Cambridge University Press.

SANAdGOL, D. 2006. Active Control of Surge in Centrifugal Compressors Using Magnetic Thrust Bearing Actuation. Ph.D. Dissertation, University of Virginia.

Scherer, C., Gahinet, P., \& Chilali, M. 1997. Multiobjective output-feedback control via LMI optimization. IEEE Trans. Autom. Contr., 42(7), 896-911.

Schmied, J., NiJhuis, A.B.M., \& Schultz, R.R. 1999. Rotordynamic Design Considerations for the 23MW NAM-GLT Compressor with Magnetic Bearings. In: Proc. IMechE Fluid Machinery Symp. The Hague.

Schonhoff, U. 2002. Practical Robust Control of Mechatronic Systems with Structural Flexibilities. Ph.D. Dissertation, Darmstadt Technical University. 
Schonhoff, U., Ehmann, C., \& Nordmann, R. 2002. Design of a robust PID-like $\mu$-synthesis controller for position control. Pages 1165-1176 of: Proc. Intl. Symp. Active Control Snd. Vib., vol. 2.

Schroder, U. 1995 (August). Section 14: Power Amplifiers. University of Virginia Magnetic Bearing Short Course, Alexandria, Virginia.

Schweitzer, G. 2002. Active Magnetic Bearings - Chances and Limitations. In: Proc. 6th Intl. IFToMM Conf. Rotordyn.

Schweitzer, G., \& Maslen, E.H. (eds). 2009. Magnetic Bearings: Theory, Design, and Application to Rotating Machinery. Springer.

Schweitzer, G., Traxler, A., \& Bleuler, H. 1994. Active Magnetic Bearings: Basics, Properties and Applications of Active Magnetic Bearings. vdf Hochschulverlag AG an der ETH Zurich.

Shahroudi, K.E., \& Young, P.M. 2008. Servocontrol of the GS16 turbine gas metering valve by physics-based robust controller synthesis. ASME J. Dyn. Sys., Meas., Control, 130(5), 051008 (9 pages).

SIDI, M. 2001. Design of Robust Control Systems: From Classical to Modern Practical Approaches. Malabar, Florida: Krieger Publishing Company.

Skogestad, S., \& Postlethwaite, I. 2005. Multivariable Feedback Control: Analysis and Design. John Wiley \& Sons.

SMith, M.C. 1990. Well-possedness of H-infinity optimal control problems. SIAM J. of Control and Optim., 28(2), 342-358.

Smith, R.D. 1995. Robust Compensation of Actively Controlled Bearings: A Performance Comparison of Regulation Methods. Ph.D. Dissertation, University of Texas at Austin.

Spakovizky, Z.S., PAduano, J.D., Larsonneur, R., Traver, A. E., \& Bright, M.M. 2000. Tip-clearance actuation with magnetic bearings for high-speed compressor stall control. In: ASME Turbo Expo.

StEIN, G. 2003. Respect the unstable. IEEE Control Sys. Mag., 23(4), 12-25.

Stein, G., \& Doyle, J.C. 1991. Beyond singular values and loop shapes. AIAA J. Guidance, 14(1), 5-16. 
Steinbuch, M., van Gross, P.J.M., Schootstra, G., Wortelboer P.M.R., \& BosGRA, O.H. 1998. mu-Synthesis for a compact disc player. International Journal of Robust and Nonlinear Control, 8, 169-189.

STEPHENS, L.S. 1995. Design and Control of Active Magnetic Bearings for a High Speed Machining Spindle. Ph.D. Dissertation, University of Virginia.

SwanN, M. 2009. Ramping up magnetic bearing use. Turbomachinery International, 50(7).

Swanson, E.E., Maslen, E.H., Li, G., \& Cloud, C.H. 2008. Rotordynamic design audits of AMB supported turbomachinery. Pages 133-158 of: Proc. 37th Turbomachinery Symp., September 8-11 2008. Houston, Texas.

TASKER, F.A., \& CHOPRA, I. 1990. Assessment of transient analysis techniques for rotor stability testing. J. Am. Helicopter Soc., 35(1), 39-50.

The MathworKs. 2009. Robust Control Toolbox 3.4 (R2009b).

TONDL, A. 1991. Quenching of self-excited vibrations. Elsevier.

Traxler, A., \& Maslen, E.H. 2009. Magnetic Bearings: Theory, Design, and Application to Rotating Machinery. Springer-Verlag. Chap. Hardware Components, pages 69-109.

Ulbrich, H. 1988. New test techniques using magnetic bearings. Pages 281-288 of: Proc. $1^{\text {st }}$ Int. Symp. Magn. Brgs. ISMB1.

van de Wal, M., van BaArs, G., Sperling, F., \& Bosgra, O. 2002. Multivariable H-infinity / mu feedback control design for high-precision wafer stage motion. Control Engineering Practice, 10, 739-755.

VAn Der Schaft, A.J., \& MAschKe, B.M. 2009. Model-Based Control; Bridging Rigorous Theory and Advanced Technology. Springer. Chap. Conservation Laws and Lumped System Dynamics, pages 31-48.

VAnCE, J., Zeidan, F., \& MurPhy, B. 2010. Machinery Vibration and Rotordynamics. Wiley.

Vinnicombe, G. 1993. Measuring the Robustness of Feedback Systems. Ph.D. thesis, University of Cambridge. 
WACHEL, J.C., \& VON Nimitz, W.W. 1981. Ensuring the reliability of offshore gas compression systems. J. Petrol. Techn., 33(11), 2252-2260.

Wagner, N.G., StefF, K., Gausmann, R., \& Schmidt, M. 2009. Investigations on the dynamic coefficients of impeller eye labyrinth seals. Pages 53-70 of: Proc. $38^{\text {th }}$ Turbomach. Symp.

Williams, R.D., Keith, F.J., \& AllaiRe, P.E. 1990. Digital control of active magnetic bearings. IEEE Tras. Ind. Electron., 37(1), 19-27.

Williams, R.D., WAyner, P.M., Ebert, J.A., \& FedigAn, S.J. 1994. Reliable, highspeed digital control for magnetic bearings. In: Proc. 4th Int. Symp. Magn. Bearings.

Wurmsdobler, P. 1997. State Space Adaptive Control for a Rigid Rotor Suspended in Active Magnetic Bearings. Ph.D. Dissertation, University of Technology Vienna.

Wurmsdobler, P., Jorgl, H.P., \& Springer, H. 1996. State space adaptive control for a rigid rotor suspended in active magnetic bearings. In: Proc. $5^{\text {th }}$ Int. Symp. Magn. Brgs. ISMB5.

YATES, S.W., \& Williams, R.D. 1988. A fault tolerant multiprocessor controller for magnetic bearings. IEEE Micro, 8(4), 6-17.

Yoon, S.Y., Lin, Z., Goyne, C., \& Allaire, P.E. 2010. Control of compressor surge with active magnetic bearings. Pages 4323-4328 of: Proc. 49th IEEE Conf. Dec. and Contr.

Young, P.M. 1996. Controller design with real parametric uncertainty. Int. J. Control, 65(3), 469-509.

ZAMES, G., \& EL-SAKKARY, A.K. 1980 (October). Unstable sytems and feedback: The gap metric. In: Proc. 16th Allerton Conf.

Zhou, K., Doyle, J.C., \& Glover, K. 1996. Robust and Optimal Control. Prentice Hall.

ZHU, L., \& KNOSPE, C.R. 2010. Modeling of nonlaminated electromagnetic suspension systems. IEEE/ASME Trans. Mechatronics, 15(1), 59-69. 
Appendix A

\section{Test Rig Functional Overview}




\section{Test Rig Functional Overview}

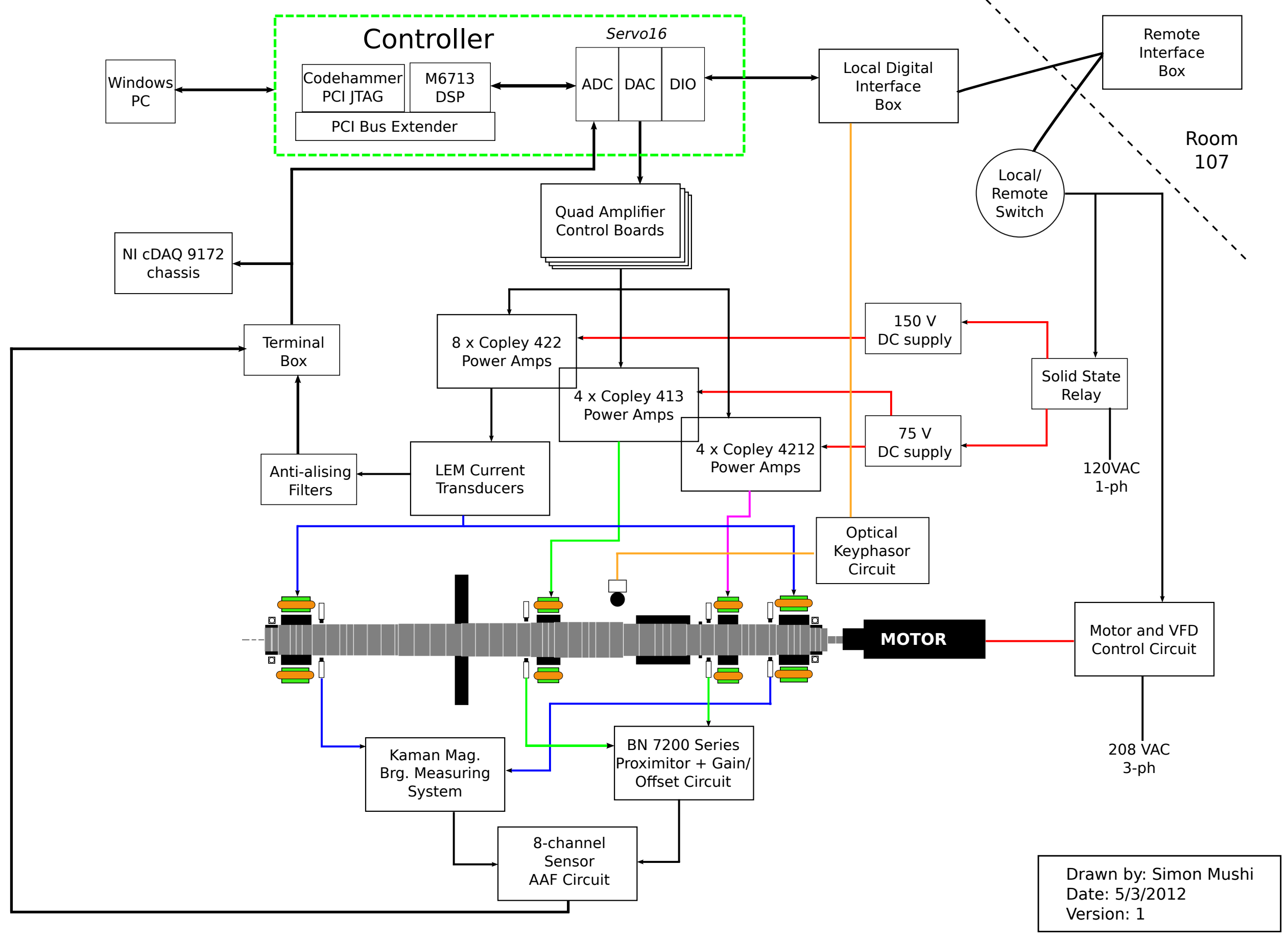




\section{Appendix B}

\section{v-Gap Metric for Robustness Analysis}

The Vinnicombe (or v-gap) metric is an easily computed measure which quantifies the difference in closed-loop performance between model sets based on their open-loop properties. A precise demonstration of the idea of this metric follows from the statements (Vinnicombe, 1993):

i) Given a nominal plant $P_{1}$, a controller $K$ and a number $\beta$, then: $\left[P_{2}, K\right]$ is stable for all plants, $P_{2}$, satisfying $\delta_{v}\left(P_{1}, P_{2}\right) \leq \beta$ iff, $b_{P_{1, K}}>\beta$.

ii) Given a nominal plant $P_{1}$, a perturbed plant $P_{2}$, and a number $\beta<b_{\text {opt }}\left(P_{1}\right)$ then: $\left[P_{2}, K\right]$ is stable for all compensators, $C$, satisfying $b_{P_{1, K}}>\beta$ iff, $\delta_{v}\left(P_{1}, P_{2}\right) \leq \beta$, where $b_{\text {opt }}\left(P_{1}\right):=\sup _{K} b_{P_{1}, K}$ is the optimal stability radius (equivalent to worst case or $H_{\infty}$ gain of the nominal closed-loop system. Any LTI plant model $P_{2}$ at a distance less than $\beta$ from the nominal plant $P_{1}$ will be simultaneously robustly stabilized by a controller $K$ which stabilizes the nominal plant with a stability margin of $\beta$. While any $P_{2}$ at a distance greater than $\beta$ from $P_{1}$, will be destabilized by any $K$ which stabilizes $P_{1}$ with a margin of at least $\beta$. A $v$-gap close to zero indicates that a given controller $K$ that robustly stabilizes $P_{1}$ will simultaneously robustly stabilize $P_{2}$, while a v-gap close to unity indicates the opposite. The $v$-gap metric is an extension of the gap metric introduced by Zames \& ElSakkary (1980); El-Sakkary (1985). The advantage of the v-gap over the standard gap 
metric is that it provides a clearer interpretation for analysis in the frequency domain, and can be computed in a point-wise manner.

The $v$-gap provides an intuitive and necessary and sufficient condition for the robust stability of two closed-loop systems given their open-loop character. However, the metric does not provide any information about the nominal or robust performance of the closedloop system. The Matlab function gap $=$ nugap $($ sys 1, sys2, ttol), calculates the $v$-gap metric for two systems sys 1 and sys2 up to a tolerance of ttol. 


\section{Appendix C}

\section{Experimental Stability Measurements}

\section{C.1 Hardware Setup}

Unidirectional and circular sinusoidal blocking excitation waveforms were generated by the same 'C6713 DSP used to execute the control law suspending the rotor. The sinusoidal waveforms are produced from a 6000 point look-up table. The amplitude, offset and frequency can be specified by the user through a graphical interface.

The voltage representing the excitation is used to drive four Copley 4212 power amplifiers, each of which was connected a single control quadrant of the quarterspan AMB. The DC gain and small-signal bandwidth of the amplifiers were adjusted to the provide a similar response for all four quadrants.

The rotor response resulting from the blocking excitation measured at all (eight) displacement sensors as well as the four controller output voltages was acquired using three NI 9234 4-channel analog input modules located in a National Instruments cDAQ 9172 8slot chassis. A digital I/O module (NI 9401) was used to receive trigger information from the DSP marking the start or end of an excitation event. A Labview VI was composed to interactively display the power spectral densities of the displacement and controller output voltages, as well as record this data for post-processing. 


\section{C.2 Damping Ratio Estimation Algorithm}

The single channel backward auto-regression algorithm to estimate damped sinusoids in a noisy background was due to Kumaresan \& Tufts (1982). A linear prediction equation was setup using the free vibration decay data $y_{i}$ collected at fixed sample rate $f_{s}$ from $i$ sensor channels. Off-line backward auto-regression uses the future values to predict the past vibration amplitudes with minimal square error and provides an intrinsic method to distinguish poles (damped eigenvalues of the system) that are due to noise from poles that are due to the physical vibration response rotor. The vibration response of the past measurements is modeled as a linear combination of the future measurements. To begin, a $q^{\text {th }}$ order difference model for each channel at a given time instant $k$ was constructed from $n$ recorded samples

$$
\begin{aligned}
y_{i}[k] & =\sum_{j=1}^{q} a_{j} y_{i}[k+i]+\varepsilon[k], \\
& =a_{1} y_{i}[k+1]+a_{2} y_{i}[k+2]+\ldots+a_{q} y_{i}[k+q]+\varepsilon[k],
\end{aligned}
$$

where $\varepsilon[k]$ is the statistical prediction error (assumed to be distributed as zero mean white noise) and $q$ is the number of unknown scalar parameters $a_{j}$ representing the dynamics of the system. The following time series linear matrix equation was constructed to solve for $a_{j}$

$$
\left[\begin{array}{llll}
a_{1} & a_{2} & \ldots & a_{q}
\end{array}\right]\left[\begin{array}{cccc}
y_{i}[1] & y_{i}[2] & \ldots & y_{i}[n-q] \\
y_{i}[2] & y_{i}[3] & \ldots & y_{i}[n-q+1] \\
\vdots & \vdots & & \vdots \\
y_{i}[q] & y_{i}[q+1] & \ldots & y_{i}[n-1]
\end{array}\right]=\left[\begin{array}{c}
y_{i}[0] \\
y_{i}[1] \\
\vdots \\
y_{i}[n-q-1]
\end{array}\right]_{(\mathrm{C} .3)}=\mathbb{Y}_{b}
$$


The above system was solved using a truncated singular value decomposition (SVD) to segregate the noise and signal subspaces. This enables lightly damped poles in a noisy background to be more easily identified than a direct application of least squares, i.e., $\widehat{\mathbb{A}}=\mathbb{Y}_{\mathrm{b}} \mathbb{Y}^{+}$. The truncated SVD allows the $r$ largest singular values of the regression matrix $\mathbb{Y}$ to be retained in a new matrix $\mathbb{Y}_{\hat{r}}$

$$
\mathbb{U}_{\hat{r}} \sum_{\hat{r}} \mathbb{V}_{\hat{r}}^{\mathrm{T}}=\mathbb{Y}_{\hat{r}}
$$

The model order $q$ needs to be several times larger than $r$, i.e., over-specified, to produce a good fit. The solution to Equation (C.4) is

$$
\hat{\mathbb{A}}=\left(-\mathbb{V}_{\hat{r}}^{\mathrm{T}} \Sigma_{\hat{r}}^{-1} \mathbb{U}_{\hat{r}}^{\mathrm{T}}\right) \mathbb{Y}_{b}
$$

The entries in $\widehat{\mathbb{A}}$ are then used to define a $q^{\text {th }}$ order characteristic polynomial

$$
B(z)=1+\hat{a}_{1} z^{-1}+\hat{a}_{2} z^{-2} \ldots+\hat{a}_{q} z^{-q} .
$$

Solving for the complex roots of $B(z)$ produces $q$ roots some of which represent the physical vibration response and others which are not. Physical modes produce roots outside the unit circle of the complex plane while roots within the unit circle are considered erroneous. Denoting the $m^{\text {th }}$ complex root of $B(z)$ located outside the unit circle as $w_{m}$, the damped eigenvalues $\lambda_{m}$ are found

$$
\lambda_{m}=-f_{s} \ln \left(w_{m}^{*}\right),
$$

and the resulting damping ratio

$$
\zeta_{m}=\frac{-\operatorname{Re}\left(\lambda_{m}\right)}{\left|\lambda_{m}\right|}
$$


and the logarithmic decrement

$$
\delta_{m}=-2 \pi \frac{\operatorname{Real}\left(\lambda_{m}\right)}{\left|\operatorname{Imag}\left(\lambda_{m}\right)\right|} .
$$

Four calculated of logarithmic decrements of $\mathrm{Nc1}$ are obtained from performing the damping ratio estimation on the NDE-X, DE-X, NDE-Y and DE-Y sensor measurements. The decay measurement was repeated five times, to yield a total of 20 data points for each CCS magnitude investigated. The statistics computed from this data are shown in Figures 6.10a and $b$. 